\title{
SEARCHES FOR NEW QUARKS AND LEPTONS IN Z BOSON DECAYS
}

\author{
Richard J. Van Kooten \\ Stanford Linear Accelerator Center \\ Stanford Univeroity \\ Stanford, Caïfornia 94309
}

June 1990

Prepared for the Department of Energy under contract number DE-ACO\&-76SF0J515

Printed in the United States of America. Available from the National Technical Information Service, U.S. Department of Commerce, 5285 Port Royal Road, Springfield, Virginia 22161. Price: Printed Copy A08, Microfiche A01.

\footnotetext{
* Ph.D. theiv
}

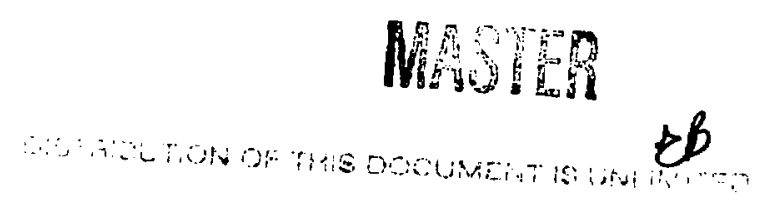




\section{Abstract}

Searches for the decay of $Z$ bosons into pairs of new quarks and leptons in a data sample including $\mathbf{4 5 5}$ hadronic $Z$ decays are presented. The $Z$ bosons were produced in electon-positron annibilations at the SLAC Linear Collider (SLC) operating in the centerof-mass energy range from 89.2 to $93.0 \mathrm{GeV}$, and the data collected using the Mark II detector.

The Standard Model provides no prediction for fermion masses and does not exclude new generations of fermions. The existence and masses of these new particles may provide valuable information to help understand the pattern of fermion masses, the presence of generations, and physics beyond the Standard Model.

Specific searches for top quarks and sequential fourth generation charge $-1 / 3\left(b^{\prime}\right)$ quarks are made considering a variety of possible standard and non-standard decay modes. In addition, searches for sequential fourth generation massive neutrinos $\nu_{4}$ (Dirac and Majorana) and their charged lepton partners $\mathrm{L}^{-}$are pursued. The $\nu_{4}$ may be stable or decay through mixing to the lighter generations. The data sample is examined for new particle topologies of events with high-momentum isolated tracks, high-energy isolated photons, spherical event shapes, and detached vertices. Measurements of the $Z$ boson resonance parameters that provide crucial indicators of new particle production are also considered.

No evidence is observed for the production of new quarks and leptons. $95 \%$ confidence lower mass limits of $40.7 \mathrm{GeV} / c^{2}$ for the top quark and $42.0 \mathrm{GeV} / c^{2}$ for the $b^{\prime}$-quark mass are obtained regardless of the branching fractions to the considered decay modes. A significant range of mixing matrix elements of $\nu_{4}$ to other generation neutrinos for a $\nu_{4}$ mass from $1 \mathrm{GeV} / c^{2}$ to $43 \mathrm{GeV} / c^{2}$ is excluded at $95 \%$ confidence level. Measurements of the upper limit of the invisible width of the $Z$ exclude additional values of the $\nu_{4}$ mass and mixing matrix elements, and also permit the exclusion of a region in the $L^{-}$mass versus $\nu_{4}$ mass plane. 
These results substantially extend previous limits, and exclude a large fraction of the mass range available for $Z$ decays into top quarks and fourth generation quarks and leptons. 


\section{Acknowledgements}

It is a pleasure to thank my advisor, Jonathan Dorfar, for guidance, valuabie advice, anc support during my graduate studies at Stanford. I an also grateful to Gary Feldman and David Burke for the experience of working with them and learning from them.

This thesis analysis benefited greatly from illumingting and belpful discussions with several people. The enthusiasm and energy of Tim Barklow, the knowledge of Sachio Komamiya, and the wisdom of a close friend, Chang-Kee Jung have been a source of inspiration for me.

I would like to thank the Mark II collaborators for their contributions during the sometimes frustrating operation of the Mark II detector at the SLC. I am just as grateful for the camaraderie and friendship of my colleagues. My office mates Carrie Fordham and graduate student (now post-doc) extraordinaire, Jordan Nash, provided many interesting conversations and company. Dave Coupal, Kathy O'Shaughnessy, Paul Dauncey, Chris Hearty, Tricia Rankin, Dean Karlen, Bob Jacobsen, Fred Kral, David Stoker, Brian Harral, Don Fujino, Eric Soderstrom, Andrew Weir, and others too numerous to name have all made for a great experience. I extend my thanks to Anna Pacheco for the countless times she willingly watched my daughter as I rushed around to finish this endeavor. Chasing the rear wheel of Sterling Watson's bicycle up to Skyline, Dirk's Jerks intramural teams, late-night poker, and the SLAC Colliders volleyball team and weekly pick-up games were enjoyable diversions.

I thank my family, personal friends, and neighbours who have given moral support over my many years as a student. A warm thanks to David, Cathy, and Alexandra Tarlinton for sharing holidays, terrific meals, Australia, and the entire Stanford experience. I am especially grateful to my wife, Mary, who provided love, friendship, and a beautiful daughter. To the best part of graduate school, our daughter Caitlin, whose smile and love made it all bearable and fun. 
I dedicate this thesis to the memory of my father who quietly instilled in me a sense of curiousity and a love of science. 


\section{Contents}

Abstract

Acknowledgements $\quad$ iv

1 Introduction 1

1.1 The Standard Model . . . . . . . . . . . . . . . . . . . 1

1.2 The Generation Puzzle . . . . . . . . . . . . . . . . . 3

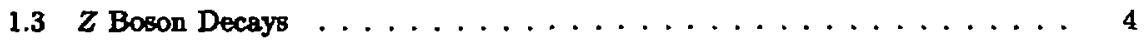

1.4 Searching for New Quarks and Leptons $\ldots \ldots \ldots \ldots \ldots \ldots$

1.5 Outline of Thesis . . . . . . . . . . . . . . . 6

2 New Quarks and Leptons in $Z$ Decays 7

$2.1 \mathrm{e}^{+} \mathrm{e}^{-} \rightarrow$ Massive Fermions $\ldots \ldots \ldots \ldots \ldots \ldots \ldots \ldots$

2.1.1 Standard Model Couplings ................ 7

2.1 .2 Lowest Order Expressions . . . . . . . . . . . . . . . . g

2.1.3 Higher Order Corrections ................. 12

2.2 The Top Quark $(t) \ldots \ldots \ldots \ldots \ldots \ldots$

2.2.1 Why the t-quark Must Exist ................... 21

2.2.2 Heavy Quark Fragmentation ... . . . . . . . . . . . 22

2.2 .3 Charged Current Decays . . . . . . . . . . . . . . 23

2.2.4 Decays into a Charged Higgs . . . . . . . . . . . . . . 24

2.2 .5 Present Mass Limits . . . . . . . . . . . . . . . . . 25

2.3 Fourth Generation $Q=-1 / 3$ Quark $\left(b^{\prime}\right) \ldots \ldots \ldots \ldots$

2.3 .1 Decay Modes . . . . . . . . . . . . . . . . . . 26

2.3.2 Present Mass Limits ... . . . . . . . . . . . . . . . . . . 28

2.4 Heavy Neutral Lepton $\left(\right.$ Neutrino, $\left.\nu_{4}\right) \ldots \ldots \ldots \ldots$ 
2.4 .1 Neutrino Mass and Mixing . . . . . . . . . . . . 29

2.4.2 Dirac and Majorana Type Neutrinos . . . . . . . . . . 30

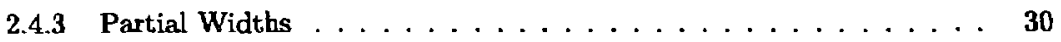

2.4 .4 Angular Distribution . . . . . . . . . . . . . . 32

2.4 .5 Decay . . . . . . . . . . . . . . . . . 32

2.4 .6 Present Mass Limits . . . . . . . . . . . . . . 36

2.5 Heavy Charged Lepton $\left(L^{-}\right) \ldots \ldots \ldots \ldots \ldots \ldots \ldots$

2.5.1 Present Mass Limits . . . . . . . . . . . . . . . . . 37

3 Experimental Apparatus

3.1 The SLAC Linear Collider (SLC) . . . . . . . . . . . . . . 38

3.1 .1 Description . . . . . . . . . . . . . . . 39

3.1.2 Machine Backgrounds . . . . . . . . . . . . . . . 41

3.1.3 Extraction Line Energy Spectrometers . . . . . . . . . . . . . 42

3.2 The Mark II Detector at the SLC . . . . . . . . . . . . . . . . . . . . . . . 44

3.2 .1 Overview . . . . . . . . . . . . . . . . 44

3.2 .2 Drift Chamber $\ldots \ldots \ldots \ldots \ldots . \ldots \ldots$

3.2 .3 Calorimetry . . . . . . . . . . . . . . . . 50

3.2 .4 Luminosity Monitors . . . . . . . . . . . . . . . . 56 56

3.2 .5 Trigger System . . . . . . . . . . . . . . 60

3.2.6 Data Acquisition System . . . . . . . . . . . . 62

4 Monte Carlo Simulations 65

4.1 Monte Carlo Event Generators $(Z \rightarrow q \bar{q}, q=u, d, s, c, b) \ldots \ldots \ldots 6$

4.1.1 LUND (JETSET 6.3) . . . . . . . . . . . . 66

4.1.2 Webber (BIGWIG 4.1) $\ldots \ldots \ldots \ldots \ldots$

4.2 Implementation of New Particles . . . . . . . . . . . . . . 69

4.2.1 $t$ and $b^{\prime}$ with Charged Current and $H^{ \pm}$Decays . . . . . . . 69

$4.2 .2 b^{\prime}$ with FCNC Decays $\ldots \ldots \ldots \ldots \ldots$

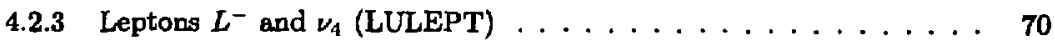

4.3 Mark II Detector Simulation $\ldots \ldots \ldots \ldots \ldots$

5 Event Selection and Analysis $\quad 72$

5.1 General Event Selection . . . . . . . . . . . . . . . . 73

5.1.1 Charged Track Requirements ...............73 
5.1 Neutral Shower Requirements . . . . . . . . . . . . 75

5.1.3 General Event Topology Requirements . . . . . . . . . . . . 76

5.1.4 Systematic Errors ... . . . . . . . . . . . . . 77

5.2 Isolated Track Topology $\ldots \ldots \ldots \ldots \ldots \ldots \ldots$

5.2.1 Expected Siguature and Background . . . . . . . . . . . . 78

$\mathbf{5 . 2 . 2}$ Topology Criteria: $\rho$ Parameter . . . . . . . . . . . . 79

5.2 .3 Efficiencies . . . . . . . . . . . . . . . . 81

5.3 Isolated Photon Topology $\ldots \ldots \ldots \ldots \ldots \ldots$

5.3.1 Expected Signature and Background . . . . . . . . . . . 85

5.3.2 Topology Criteria: a, Parameter . . . . . . . . . . . 85

5.3.3 Efficiencies . . . . . . . . . . . . . . . . 86

5.4 Spherical Event Topology . . . . . . . . . . . . . . . . . 87

5.4.1 Expected Signature and Background ... . . . . . . . . 87

5.4.2 Topology Criteris: Mass out of the Plane . . . . . . . . . . . 88

5.4 .3 Efficiencies $\ldots \ldots \ldots \ldots \ldots \ldots$

5.5 Combined Analysis for $b^{\prime} \ldots \ldots \ldots \ldots \ldots \ldots \ldots \ldots$

5.6 Detached Vertex Topology . . . . . . . . . . . . . . . . 93

5.6.1 Expected Signature and Background . . . . . . . . . . . . . 93

5.6.2 Topology Criteria: Normalized Impact Parameter Method . . . . . . 94

5.6 .3 Efficiencies . . . . . . . . . . . . . . . . . . 99

5.6 .4 Systematic Errors . . . . . . . . . . . . . . . . 101

5.7 Mass Limits from Measurements of the $Z$ Resonance . . . . . . . . . . . 102

5.7 .1 Stable $\nu_{4} \ldots \ldots \ldots \ldots \ldots \ldots \ldots \ldots \ldots$

5.7 .2 Visible Event Selection Criteria . . . . . . . . . . . . . 103

5.7 .3 Unstable $\nu_{4} \ldots \ldots \ldots \ldots \ldots \ldots$. . . . . . . . . . . . 105

5.7.4 Heavy Charged Lepton $L^{-} \ldots \ldots \ldots \ldots$. . . . . . . . . 107

6 Results $\quad 110$

6.1 Expected Number of Events . . . . . . . . . . . . . . . . 110

6.1.1 Number of Produced Events . . . . . . . . . . . . . . 110

6.1.2 Errors on the Number of Produced Events . . . . . . . . . . . . . 112

6.1.3 Expected Number of Events after Cuts . . . . . . . . . . . . . 114

6.2 Mass Limits and Exclusion Regions (Direct Searches) . . . . . . . . . . 114

6.2 .1 Isslated Track Topology . . . . . . . . . . . . . . . . . . 115 
6.2 .2 Isolated Photon Topology . . . . . . . . . . . . . . . . . . 120

6.2.3 Spherical Event Topology . . . . . . . . . . . . . . . 121

6.2.4 Combined Analysis for $b^{\prime} \ldots \ldots \ldots \ldots \ldots$

6.2.5 Detached Vertex Topology . . . . . . . . . . . . . . . . 125

6.3 Limits from Measurements of $Z$ Resonance Parameters . . . . . . . . . . . 127

6.3.1 Z Resonance Fit Results . . . . . . . . . . . . . . . 127

6.3.2 Stable $\nu_{4}$ Mass Limits . . . . . . . . . . . . . . . . . . . 128

6.3.3 Unstable $\nu_{4}$ Limits . . . . . . . . . . . . . . . 131

6.3.4 Heavy Charged Lepton $L^{-}$Limits . . . . . . . . . . . . . 132

6.4 Summary and Comparisons .................... 133

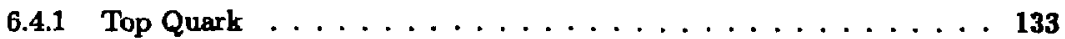

6.4.2 Fourth Generation $b^{\prime}$-Quark . . . . . . . . . . . . . 135

6.4 .3 Fourth Generation Neutrino $\nu_{4} \ldots \ldots . \ldots . \ldots . . \ldots 137$

6.4.4 Fourth Generation Charged Lepton $L^{-} \ldots \ldots \ldots$. . . . . . . 137

6.5 Conclusions . . . . . . . . . . . . . . . . . . 139 


\section{List of Tables}

1 The fundamental fermions. $\ldots \ldots \ldots \ldots \ldots \ldots \ldots \ldots \ldots$

2 The members of a possible fourth generation of new quarks and leptons. . 5

3 Arrangement of fermions into isodoublets and isosinglets. . . . . 8

4 Neutral current coupling constants. . . . . . . . . . . . 9

5 Partial widths of $Z$ to the known fundamental fermions. . . . . . . . 19

6 SLC Machine Parameters . . . . . . . . . . . . . . 41

7 Monte Carlo Parameters . . . . . . . . . . . . . . . . 68

8 Effect of cuts on efficiencies. . . . . . . . . . . . . 83

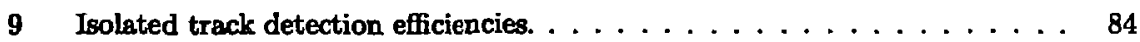

10 Detection efficiencies for $b^{\prime} \rightarrow b \gamma \ldots \ldots \ldots \ldots \ldots \ldots \ldots$

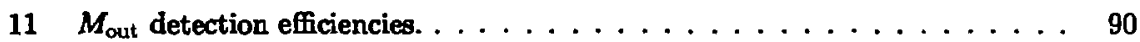

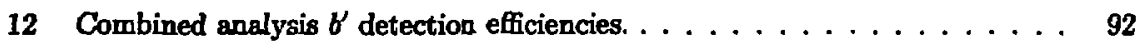

13 Normalized impact parameter detection efficiencies. . . . . . . . . . 100

14 Efficiencies for $\nu_{4}$ events to pass visible event criteria. . . . . . . . 107

15 Efficiencies for new particle events to pass hadronic event cuts. . . . . 113

16 Mess limits from the isolated track analysis for prompt $\nu_{4} \ldots \ldots \ldots \ldots 119$

17 Mass ranges excluded by $M_{\text {out }}$ analysis. $\ldots \ldots \ldots \ldots \ldots \ldots \ldots \ldots$

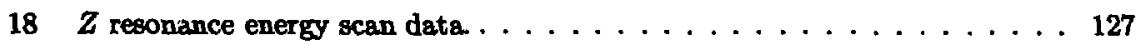




\section{List of Figures}

1 Masses of the known fundamental fermions. . . . . . . . . . . 3

$2 e^{+} e^{-}$cross section as a function of center-of-mass energy. . . . . . . . 4

3 Feynman diagram for $Z$ decay. . . . . . . . . . . . . . 10

4 Feynman diagrams describing the process of fermion production through $e^{+} e^{-}$annihilation. . . . . . . . . . . . . . 11

5 Examples of cross sections as a function of new particle mass. . . . . . . 13

6 Feynman diagram describing final state gluon radiation in hadronic $Z$ decay. 14

$7 \quad$ Feynman diagram describing final state photon radiation in $Z$ decay. . . . . 16

8 Feynman diagroms describing oblique or internal loop radiative corrections. 16

9 Feynman diagrams describing radiative corrections for the $Z-b \bar{b}$ vertex. . . 18

10 Partíal widths for new heavy fermions. . . . . . . . . . . . . . . 20

11 Uncertainty in heavy quark partial widths. . . . . . . . . . . 20

12 Fragmentation of heavy quark to heavy hadron. . . . . . . . . . . . 22

13 Decay of a top hadron and top quark. . . . . . . . . . . . . 23

14 Feynman diagram describing decay of $t$ through a real charged Higgs $\mathrm{H}^{+}$. . 24

15 Charged current and fiavor-changing neutral-current decays of the $b^{\prime}$-quark. 27

16 Partial widths for Dirac and Majorana neutrinos. . . . . . . . . . . 31

17 Feynman diagrams describing allowed and suppressed decays of massive Dirac neutrinos. ...................... 33

18 Possible Majorana neutrino decay modes. . . . . . . . . . . . . 34

19 Mean decay lengths of massive neutrinos. . . . . . . . . . . 35

20 Present mass limits for $\nu_{4}\left(100 \%\right.$ mixing to $\left.\nu_{e}\right) \ldots \ldots \ldots \ldots$

21 Schematic layout and operation of the SLC . . . . . . . . . . . . 40

22 Schematic layout of the energy spectrometer. . . . . . . . . . . . . . 42

23 The phosphorescent screen monitor (PSM) . . . . . . . . . . . 43 


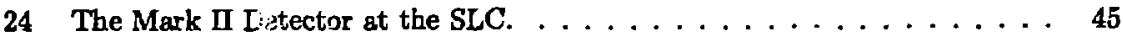

25 Drift cbamber wire configuration. . . . . . . . . . . . . . 47

26 Track reconstruction efficiency. . . . . . . . . . . . . . . . . 49

27 Two-track separation efficiency for the drift chamber. . . . . . . . . 50

28 Electromagnetic calorimetric coverage. . . . . . . . . . . . . . 51

29 Ganging of liquid argon calorimeter channels. . . . . . . . . . . 52

30 Measured LA energy distribution for Bhabhas. . . . . . . . . . . . . 53

31 Monte Carlo siniulation of liquid argon energy resolution. . . . . . . . . 54

32 Response of the endcap calorimeter to between one and five $10 \mathrm{GeV}$ positrons. 55

33 Layout of luminosity monitors. . . . . . . . . . . . . . . 57

34 Layout of the Small-Angle Monitor. . . . . . . . . . . . . 57

35 Geometry of the Mini-Small-Angle Monitor. . . . . . . . . . . . . 59

36 Block diagram of the charged track data trigger. . . . . . . . . . . . 61

37 Architecture of Mark II data acquisition system. . . . . . . . . . . . 63

38 Feynman diagram of the two-photon process. . . . . . . . . . . 73

39 Distributions of distance of closest appraach of tracks. . . . . . . . . . . 74

40 Transverse moments and $|\cos \theta|$ of tracks. . . . . . . . . . . 74

41 Distribution of the energy of neutral showers. . . . . . . . . . . 76

42 Distribution of the fraction $E_{\text {vis }} / E_{\mathrm{cm}} \ldots \ldots \ldots \ldots \ldots \ldots$

43 Quantities used in the determinstion of the p-parameter. . . . . . . . 81

44 Example of a Monte Carlo event with an isolated track. . . . . . . . . 82

45 Distribution of isolation parameter $p . \ldots \ldots \ldots \ldots$. . . . . . . . . 83

46 Distribution of isolation parameter $\rho_{\gamma} \ldots \ldots \ldots \ldots \ldots \ldots$

47 Schematic of $p_{T}^{\text {out }}$ as used to determine $M_{\text {out }} \ldots \ldots \ldots \ldots$

48 Distribution of $M_{\text {out }} \ldots \ldots \ldots \ldots \ldots \ldots \ldots$

49 Example of a Monte Carlo $\nu_{4} \bar{\nu}_{4}$ event. . . . . . . . . . . . . . 93

50 Distribution of primary vertices in the $x-y$ plane. . . . . . . . . 96

51 Definition of impact parameter and related errors. . . . . . . . . 97

52 Distribution of track impact parameter error. . . . . . . . . . . . . 98

53 Distribution of $v_{4}$ search parameter $\chi_{\text {imp }} \ldots \ldots \ldots \ldots \ldots$

54 Tracking efficiency as a function of missing layer information. . . . . . . . 102

55 Efficiency $\eta_{\text {vis }}$ as a function of $\nu_{4}$ lifetime. . . . . . . . . . . 106

56 Efficiency $\eta_{v i s}$ as a function of $m_{L}$ and $m_{\nu_{4}} \ldots \ldots \ldots \ldots$ 
57 Typical hadronic $Z$ decay in the data. . . . . . . . . . . 111

58 Expected number of produced new particle events. . . . . . . . . . 113

59 Expected number of $t$ and $b^{\prime}$ (CC decays) quark events passing isolated track cuts. . . . . . . . . . . . . . . . . . . 116

60 Expected number of $Z \rightarrow \nu_{A}^{D} \bar{\nu}_{4}^{D}$ events passing isolated track cuts. . . . 118

61 Comparing $N_{\exp }$ for Dirac and Majorana neutrinos. . . . . . . . . 118

62 Excluded region for $\nu_{4}$ prcduction in the $m_{\nu_{4}}$ versius $\left|U_{\ell_{4}}\right|^{2}$ plane. . . . . 119

63 Expected number of $b^{\prime} \rightarrow b \gamma$ events. . . . . . . . . . . . 120

64 Expected number of $Z \rightarrow b^{\prime} \bar{b}^{\prime}$ events with $M_{\text {out }}>2$ ) $\mathrm{GeV} / c^{2} \ldots \ldots \ldots$

65 Excluded mass ranges as a function of $\operatorname{Br}(H \rightarrow c \bar{s}) \ldots \ldots \ldots \ldots \ldots$

66 Excluded $\forall$ mass range as a function of decay mode branching fractions. . . 124

67 Number of expected long-lived $\nu_{4} \bar{\nu}_{4}$ events. . . . . . . . . . 125

$68 \nu_{4}$ exclusion regions from detached vertex topology. . . . . . . . . 126

$69 Z$ energy scan data and resonance fit. . . . . . . . . . . . . . . 129

$70 \log$ likelihood as a function of $N_{\nu} \ldots \ldots \ldots \ldots \ldots \ldots$

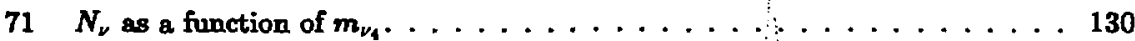

72 Maximum efficiency poesible for $\nu_{4} \bar{\nu}_{4}$ events for a limit from width. . . . . 131

$7395 \%$ exclusion regions in $m_{\nu_{4}}-\left|U_{\ell_{4}}\right|^{2}$ plane from /zee/ width . . . . . . 132

$7495 \%$ exclusion regions in $m_{L}-m_{\nu, 4}$ plane from / zee/ width. . . . . . . 133

75 Summary and comparison of $t$-quark mass limits. . . . . . . . . . 134

76 Summary and comparison of $b^{\prime}$-quark mass limita. . . . . . . . . . 136

77 Comparison of Mark II $\nu_{4}$ exclusion regions with previous and subsequent limits. . . . . . . . . . . . . . . . . . . . . . 138

78 Comparison of Mark II $m_{L}-m_{\nu_{L}}$ exclusion regions with previous and subsequent limits. 


\section{Chapter 1}

\section{Introduction}

Elementary particle physics is the study of the fundamental constituents of matter, the elementary particles, and the forces that act between them. The goal of particle physics is to discover the unifying principles and physical laws that result in a rational and predictive picture of the elementary particles and basic forces that constitute our universe.

\subsection{The Standard Model}

At the present time, it is believed that all matter is made up of pointlike, spin one-half particles (fermions) called quarks and leptons grouped into three generations or families as shown in Table i. Integral spin particles (bosons) are responsible for the four fundamental forces which act between these elementary particles. The electromagnetic force is transmitted or mediated by the massless photon $(\gamma)$ over an infinite range between particles with electric charge. The weak force acts between all particles, but over a limited range, and is mediated by the massive intermediate vector bosons $\left(W^{+}, W^{-}\right.$, and $Z$ ). The strong force operates between quarks to hold them together in quark-antiquark combinations (mesons, such as the pi meson, $\pi$ ) or three-quark combinations (baryons, such as the proton and neutron) by the exchange of particles appropriately named glwons $(g)$. All the particles which undergo strong interactions, baryons and mesons, are collectively called hadrons. Leptons do not experience the strong interaction. Finally, the gravitational force acts between all particles, but is so weak for typical distances between the elementary particles that it can effectively be ignored.

In the last two decades, great progress has been made in understanding the nature of 
the electromagnetic, weak, and strong forces. The electromagnetic and weak forces have been unified by the Electroweak theory [1] which rests upon an underlying symmetry called local gauge invariance. A neutral scalar, the Higgs boson, is included which "breaks" this symmetry and provides mass to the $W^{+}, W^{-}$, and $Z$ bosons. The combination of the Electroweak theory and the analogous gauge theory of Quantum Chromodynamics (QCD) describing the strong or colour force between quarks is known as the Standard Model. Predictions of the Standard Model have been dramatically verified by many experiments, culminating in the discovery [2] of the $W$ and $Z$ particles in 1983 near their predicted marses.

Table 1: The fundamental fermions. The six quarks are named up, down, charm, strange, top, and bottom; the three charged leptons are named the electron, muon, and tau; and the three neutral leptons are the electron neutrino, muon neutrino, and tau neutrino. There is an associated antiparticle for each particle in this table. The top quark and tau neutrino have not yet been directly observed.
Quarks
$\left(\begin{array}{l}u \\ d\end{array}\right)$
$\left(\begin{array}{l}c \\ s\end{array}\right)$
$\left(\begin{array}{c}(t) \\ b\end{array}\right)$
Electric Charge
Leptons $\quad\left(\begin{array}{c}\nu_{e} \\ e^{-}\end{array}\right)$
$\left(\begin{array}{c}\nu_{\mu} \\ \mu^{-}\end{array}\right)$
$\left(\begin{array}{c}\left(\nu_{\tau}\right) \\ \tau^{-}\end{array}\right)$
$+\frac{2}{3}$
$-\frac{1}{3}$
$1^{\text {st }}$ Generation $2^{\text {nd }}$ Generation $3^{\text {rd }}$ Generation

Despite its many successes, several troubling questions remain unanswered, indicating that the Standard Model mist be incomplete. For a fundamental theory, the Standard Model has too many free parameters including the values of the fermion masses and the three separate coupling constants for the electromagnetic, weak, and strong interactions. A complete theory would unify these interactions in a single gauge group with a single coupling through so called Grand Unified Theories (GUTs), would try to explain the particular vaiues of the fermion masses, and would offer an explanation of the "generation puazle". 


\subsection{The Generation Puzzle}

Why is there more than one generation of fermions? Protons, neutrons, and electrons constitute the matter in our everyday lives and these are composed only of the members of the first generation. Particles of successive generations generally appear only in high energy particle experiments. The reason for this bizarre replication of families is still an open question. A distinctive feature of the generations is that the fermions of each successive generation are more massive than those in the preceding ones as shown in Fig. 1.

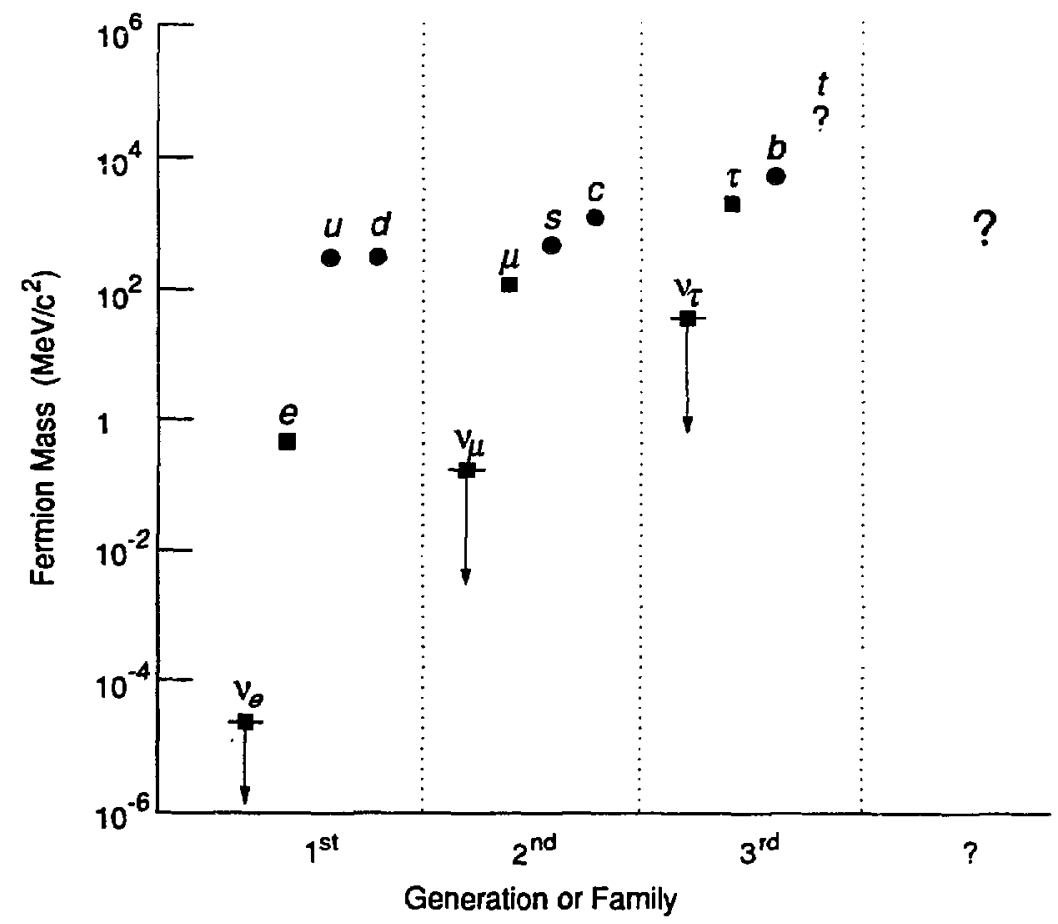

Figure 1: Masses of the known fundamental fermions, showing the mass hierarchy between quarks (constituent masses, dots) and leptons (squares). The upper limits on the masses of the neutrinos are shown.

Why do the quark and lepton masses increase with each genezation? Why are the ratios of quark masses within a family so small and the ratios of lepton masses so large? It is 
natural to search for yet another replication of even more massive fermions, a fourth family or generation, to provide clues to this proliferation of mysteries.

\subsection{Z Boson Decays}

The observation of $Z$ boson decays at rest in $e^{+} e^{-}$annihilations is an ideal environment for the study of the fundamental fermions. Firstly, the $Z$ boson provides a resonance and consequent huge enhancement in the cross section or event rate for $e^{+} e^{-}$ccilisions as shown in Fig. 2. Secondly, the $Z$ will decay into a particle-antiparticle pair of all the

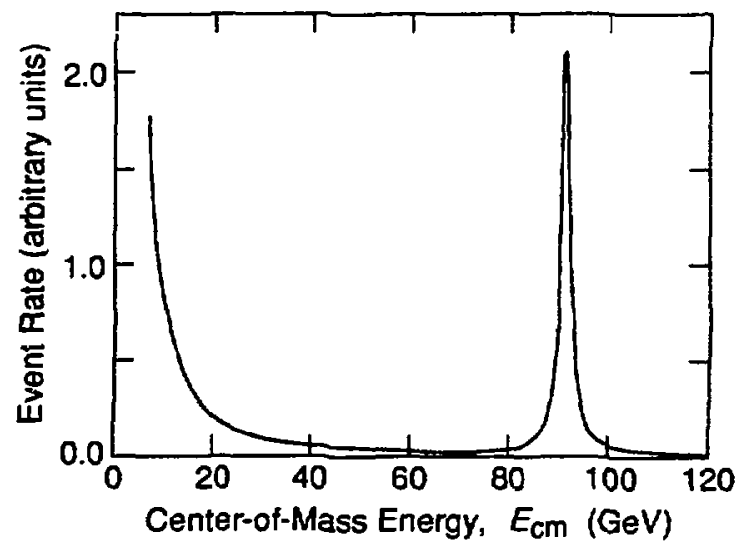

Figure 2: Relative $e^{+} e^{-}$annihilation event rate (for constant luminosity, $e^{+} e^{-} \rightarrow \mu^{+} \mu^{-}$shown as an example case) es a function of center-of-mass energy $E_{\mathrm{cm}}$. The large resonance occurs at $E_{\mathrm{cm}} \approx M_{Z} \approx 91 \mathrm{GeV}$.

known fundamental fermions listed in Table 1, plus any new fermion that has Standard Model couplings and mass less than one-half the mass of the $Z$ boson $\left(M_{Z}\right)$. Thirdly, in $e^{+} e^{-}$annihilation at center-of-mass energy $E_{\mathrm{cm}} \approx M_{Z}$, it is generally only the decay products of the $Z$ that are observed, resulting in a clean environment for the detailed study of the produced fermions and their decays. The original discovery [2] of $Z$ bosons in proton-antiproton $(p \bar{p})$ collisions identified only the decays $Z \rightarrow e^{+} e^{-}$and $Z \rightarrow \mu^{+} \mu^{-} ; e^{+} e^{-}$ annihilation permits the first identification of the additional deceys of the $Z$ to quarks. 


\subsection{Searching for New Quarks and Leptons}

The Standard Model is essentially unchanged with the addition of a fourth generation as shown in Table 2. These members of a fourth generation will be decay products of the $Z$ as long as their masses are less than $M_{Z} / 2$. This thesis presents searches using the Mark II detector for the sequential fermions $b^{\prime}, \nu_{4}$, and $L^{-}$taking into account their possible different decay modes in a data sample of 455 hadronic $Z$ decay events provided by the SLAC ${ }^{\dagger}$ Linear Collider (SLC) between April 1989 and November 1989. Since the top quark remains undiscovered, it will be searched for instead of the fourth generation $t^{\prime}$-quark. At

Table 2: The members of a possible fourth generation of new quarks and leptons. There is an associated antiparticle for each particle in this table.

Fourth Generation Quarks $\left(\begin{array}{c}t^{\prime} \\ b^{\prime}\end{array}\right) \begin{gathered}\text { Electric Charge } \\ +\frac{2}{3} \\ -\frac{1}{3}\end{gathered}$
Fourth Generation Leptons $\left(\begin{array}{c}\nu_{4} \\ L^{-}\end{array}\right)$
-1

the time of analysis, there were hints of the possibility of a fourth generation from a larger ratio of hadronic to $\mu$-pair events found at TRISTAN [3] compared to three-generation expectations. In addition, if the top quark mass is not too large, the recently measured large value of $B-\bar{B}$ mixing [4] suggests the possibility of another generation. It will be seen that despite the relatively small number of $Z$ decays collected by the Mark II detector, the only way for a top quark or a fourth generation to escape detection in $Z$ deceys would be if all the corsidered particles have masses greater than approximately $M_{Z} / 2$.

Many of the topological search techniques presented are based on the fact that the above new quarks and leptons are necessarily much heavier than the known fermions. In the decay $Z \rightarrow f \bar{f}$, fermions with small masses will have large momentum from the constraint $p_{f}=\left(E_{f}^{2}-m_{f}^{2}\right)^{1 / 2}$ where $p_{f}$ is the momentum, $m_{f}$ is the mass, and $E_{f}$ is the energy $\left(E_{f} \simeq M_{Z} / 2\right)$ of the fermion. As a result, if the produced fermion and antifermion decay, their decay products will be limited to tightly collimated cones (jets) of particles.

\footnotetext{
'Stanford Linear Accelerator Center at Stanford, California, USA.
} 
In contrast, particles from heavy fermion decay are distributed over a wider solid angle than the decay products from lighter fermions of the same energy. If both the heavy fermion and antifermion decay hadronically, their decay products are distributed rather isotropically, and a spherical event topology results which can be characterized by certain event shape parameters. Semileptonic or leptonic decay of a heavy fermion leads to at least one lepton among the decay products, and the lepton will in general be isolated from the rest of the decay products, forming a distinctive signature.

Heavy fermions such as $\nu_{4}$ can also have very long lifetimes, leading to spectacular detached vertex topologies. Finally, indirect search techniques are also used in some cases to detect the presence of new leptons. If the $Z$ can decay into new particles, its lifetime will decrease from Standard Model predictions. The measured width $\Gamma_{Z}$ of the resonant form of the total $e^{+} e^{-}$cross section for collision energies near $M_{Z}$ is directly related to this lifetime and will increase if the $Z$ decays into particles other than the known fermions.

\subsection{Outline of Thesis}

Chapter 2 is a theoretical description of the production of the $Z$ boson in $e^{+} e^{-}$annihilation and its ensuing decay into massive fermions including QCD and radiative corrections. The characteristics, decay modes, and present (at the time of the analysis) mass limits of each of tine $n \in \mathbb{w}$ quarks and leptons of interest are also discussed. A description of the experimental apparatus of the SLC and the Mark II detector is provided in Chepter 3. Chapter 4 outlines the Monte Carlo event simulation of $Z$ production and decay into the known fermions, new quarks and leptons, and their subsequent decays. Chapter 5 contains a discussion of new quark and lepton selection methods and criteria, and the efficiencies for new particle and known fermion events to satisfy the criteria. Results and mass limits on the various new particle scenarios are presented in Chapter 6. Starting in September 1989, the experiments at LEP $^{\dagger}$ started collecting $Z$ decay data. Months after the publication of most of the results of this thesis [5], the LEP experiments also published similar results using a much larger sample of $Z$ decays. Chapter 6 also includes comparisons of the Mark II limits with LEP limits.

\footnotetext{
${ }^{\dagger}$ Large Electron-positron Project, a large-scale conventional storage ring device at CERN, Geneva, Switzerland.
} 


\section{Chapter 2}

\section{New Quarks and Leptons in $Z$ Decays}

In this chapter, the process $e^{+} e^{-} \rightarrow$ (Massive fermions) is described in the framework. of the Standard Model for center-of-mass energies near the mass of the $Z$ boson $\left(M_{Z}\right)$ in order to calculate the production rates of new quarks and leptons from $Z$ decays. The relevant charscteristics, decay modes, and present (at the time of the analysis) mass limits of each considered new quark and lepton are then described.

\section{$2.1 \mathrm{e}^{+} \mathrm{e}^{-} \rightarrow$ Massive Fermions}

\subsubsection{Standard Model Couplings}

The gauge group $S U(3)_{C} \otimes S U(2)_{L} \otimes U(1)$ characterizes the Standard Model and includes the unification of the electromagnetic and weak forces into a single electroweak interaction. The mathematical structure of this theory rests upon an underlying symmetry called local gauge invariance. Through a rotation by the Weinberg angle $\theta_{W}$, the $U(1)$ field $B_{\mu}$ and $S U(2)$ field $W_{\mu}^{3}$ give rise to the mass eigenstates:

$$
\begin{aligned}
W_{\mu}^{ \pm} & =\frac{1}{\sqrt{2}}\left(W_{\mu}^{1} \pm W_{\mu}^{2}\right) \\
Z_{\mu} & =\cos \theta_{W} W_{\mu}^{3}+\sin \theta_{W} B_{\mu} \\
A_{\mu} & =-\sin \theta_{W} W_{\mu}^{3}+\cos \theta_{W}
\end{aligned}
$$

which are, respectively, the gauge bosons $W^{ \pm}, Z$, and $\gamma$ that mediate the electroweak interactions between the fermionic particles. As shown in Table 3, left-handed fermions are 
$8 \ldots$ Chapter 2. New Quarks and Leptons in Z Decays

grouped in weak isodoublets whose upper members have $T_{3}=1 / 2$ and lower members have $T_{3}=-1 / 2$ where $T_{3}$ is the third component of the weak charge or isospin. Right-handed fermions are arranged in weak isosinglets with $T_{3}=0$. If neutrinos are massless, then there are no right-handed neutrinos, and no neutrino isosinglets.

The unitary Kobayashi-Maskawa (KM) matrix [6] with complex matrix elements $V_{i j}$ :

$$
\left(\begin{array}{c}
d^{\theta} \\
s^{\theta} \\
b^{\theta}
\end{array}\right)=\left(\begin{array}{lll}
V_{u d} & V_{u s} & V_{u b} \\
V_{c d} & V_{c s} & V_{c b} \\
V_{t d} & V_{t s} & V_{t b}
\end{array}\right)\left(\begin{array}{c}
d \\
s \\
b
\end{array}\right)
$$

relates the weak eigenstates to the mass eigenstates of quarks. The weak eigenstates corresponding to the charge $-1 / 3$ quarks are written with $\theta$ superscripts ${ }^{\dagger}$ to indicate that they are not the same as the mass eigenstates. Elements of the $\mathrm{KM}$ matrix enter into calculations including weak charged current processes involving $W$ bosons.

Table 3: Arrangement of left-handed fermions into weak isodoublets and right-handed fermions into weak isosinglets.

$$
\begin{aligned}
& \left(\begin{array}{c}
\nu_{e} \\
e
\end{array}\right)_{L}\left(\begin{array}{c}
u \\
d^{\theta}
\end{array}\right)_{L}\left(\begin{array}{llll}
(e)_{R} & (u)_{R} & (d)_{R}
\end{array}\right. \\
& \left(\begin{array}{c}
\nu_{\mu} \\
\mu
\end{array}\right)_{L}\left(\begin{array}{c}
c \\
s^{\theta}
\end{array}\right)_{L}(\mu)_{R} \quad(c)_{R} \quad(s)_{R} \\
& \left(\begin{array}{c}
\nu_{\tau} \\
\tau
\end{array}\right)_{L}\left(\begin{array}{c}
t \\
b^{\theta}
\end{array}\right)_{L}(\tau)_{R} \quad(t)_{R} \quad(b)_{R}
\end{aligned}
$$

\footnotetext{
The $q^{\theta}$ notation is used instead of the usual $q^{\prime}$ notation to avoid confusion of quark weak eigenstates with fourth generation sequential quarks $b^{\prime}$ and $t^{\prime}$.
} 
Neutral current processes are represented by the vertex factors:
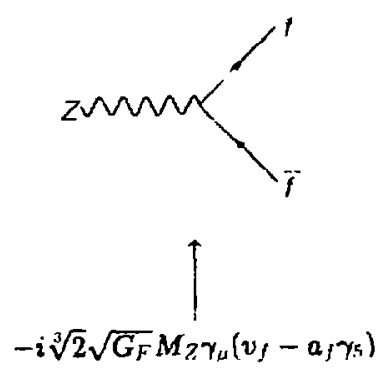
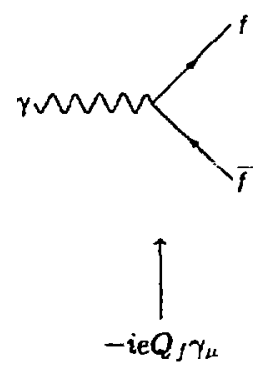

where $G_{F}$ is the Fermi constant, $Q_{f}$ is the fermion electric charge,

$$
\begin{aligned}
& a_{f}=2 T_{3}^{J} \\
& v_{f}=2\left(T_{\tau}^{\prime}+2 Q_{f} \sin ^{2} \theta_{W}\right)
\end{aligned}
$$

are the axial-vector and vector neutral coupling constants, and $\gamma_{i}$ are the gamma matrices in the usual notation [7]. The values for these constants are listed in Table 4 for the known fundamental fermions and for possible new quarks and leptons.

Table 4: Axial-vector $a_{\text {f }}$ and vector $v_{\text {f }}$ neutral current coupling constants for the known fundamental fermions and possible new quarks and leptons.

\begin{tabular}{lrrrcc}
\hline \hline$f$ & $Q_{f}$ & $T_{3 j}$ & $a_{f}$ & $v_{f}$ & New Heavy Fermion \\
\hline$\nu_{t}, \nu_{\mu}, \nu_{\tau}$ & 0 & $\frac{1}{2}$ & 1 & 1 & $\nu_{4}$ \\
$e^{-}, \mu^{-}, \tau^{-}$ & -1 & $-\frac{1}{2}$ & -1 & $-1+2 \sin ^{2} \theta_{W}$ & $L^{-}$ \\
$u, c$ & $\frac{2}{3}$ & $\frac{1}{2}$ & 1 & $1-\frac{8}{3} \sin ^{2} \theta_{W}$ & $t$ \\
$d, s, b$ & $-\frac{1}{3}$ & $-\frac{1}{2}$ & -1 & $-1+\frac{4}{3} \sin ^{2} \theta_{W}$ & $b^{\prime}$ \\
\hline
\end{tabular}

\subsubsection{Lowest Order Expressions}

In order to calculate the cross section for $e^{+} e^{-} \rightarrow f \bar{f}$, we need to first find the decay rate or width $\Gamma_{Z}$ of the $Z$ boson. We can obtain the partial decay rate of the $Z$ into a massive fermion-antifermion pair in the Born approximation (i.e. at tree-level) from the Feynman diagram of Fig. 3. The amplitude for this mode is: 


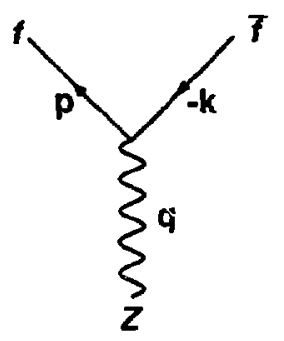

Figure 3: Feynman diagram used to calculate the decay rate of the $Z$.

$$
\mathcal{M}_{Z f \bar{f}}=-\frac{i}{\sqrt{2}}\left(\frac{G_{F} M_{Z}^{2}}{\sqrt{2}}\right)^{1 / 2} \epsilon_{\alpha}^{\lambda}(q) \bar{u}(p) \gamma^{\alpha}\left(v_{f}+a_{f} \gamma_{5}\right) v(\mathbf{k})
$$

with momenta labelled as in Fig. 3, and where the spinors $\bar{u}(\mathrm{p}), v(k)$, and polarization vector $\epsilon_{\alpha}^{\lambda}$ are defined in the usual notation [7]. The differential decay rate for the two body decay can then be written [8]:

$$
\frac{d \Gamma}{d \Omega_{\mathrm{cm}}}=\frac{|\mathcal{M}|^{2}}{64 \pi^{2}} \frac{s \beta}{M_{Z}^{3}}
$$

where $s=E_{c m}^{2}$ with $E_{c m}$ the total energy in the center-of-mass (CM) frame, $m_{f}$ is the mass of the final state fermion, $\beta=\left(1-4 m_{f}^{2} / s\right)^{1 / 2}$ is the velocity of the final state fermion in the $\mathrm{CM}$ frame, and $d \Omega_{\mathrm{cm}}$ is the differential solid angle element in the CM frame. Integrating over the solid angle, we arrive at:

$$
\Gamma^{0}(Z \rightarrow f \bar{f})=\frac{G_{F} M_{Z}^{3}}{24 \sqrt{2} \pi} \beta\left[\left(\frac{3-\beta^{2}}{2}\right) v_{f}^{2}+\beta^{2} a_{f}^{2}\right] .
$$

The total width or decay rate of the $Z$ is then simply

$$
\Gamma_{Z}^{0}=\sum_{f} \Gamma^{0}(Z \rightarrow f \bar{f}) D^{f}
$$

where $f$ ranges over all the fermions that the $Z$ is kinematically allowed to decay into $\left(m_{f}<M_{Z} / 2\right)$, and the color factor $D^{\prime}$ takes into account the three different color states for each quark. Hence, $D^{f}=3$ if $f$ is a quark, and $D^{f}=1$ if $f$ is a lepton.

The beanty of $Z$ physics is exemplifed in Eq. 6. The total width $\Gamma_{Z}$ can be measured from the resonant form of the total $e^{t} e^{-}$cross section near $s=M_{Z}^{2}$. It is an important 
window on possible new physics. Any new particle with non-trivial $S U(2) \otimes U(1)$ quantum numbers will couple to the $Z$ and appear in $Z$ decays if light enough, revealing its presence through an increase in $\Gamma_{Z}$ above Standard Model expectations. Particularly interesting are $Z$ decays into stable neutrinos which essentially do not interact in a colliding beam detector. Even though they are 'invisible' decays, their existence can be inferred from measurements of the $Z$ resonance parameter:.

We now consider the process of $e^{t} e^{-}$annihilation into a pair of massive fermions. In lowest order, this process is described by the Feynman graphs in Fig. 4. We have ignored
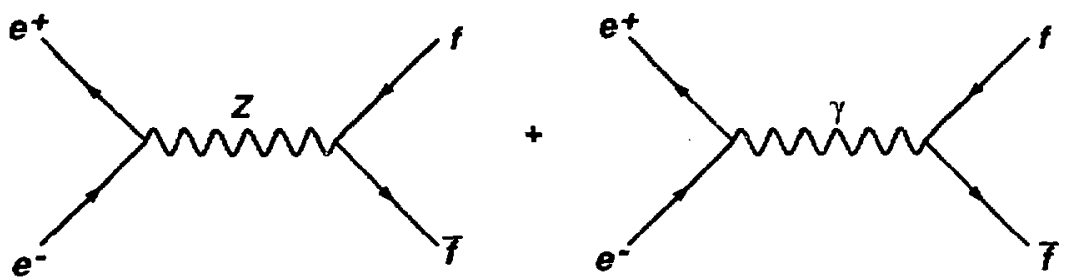

Figure 4: Feynmen diagrams describing the process of fermion production through $e^{+} e^{-}$annihilation.

t-channel diagrams which are only important at small production angles with respect to the incident beam direction. Higgs exchange can also be neglected because of the small Yukawa coupling to the electron. The corresponding Feynman amplitude is given by

$$
M=M_{\gamma}+M_{Z}
$$

Without neglecting terms from the final fermion mass $m_{f}$, the differential cross section can be written in the following way, where the color factor $D^{f}=1$ (leptons), and $D^{f}=3$ (quarks) distinguishes between the final state fermions, and $\theta$ is the polar angle between the incident electron direction and the outgoing fermion $f$ :

$$
\frac{d \sigma}{d \Omega}=\frac{\alpha^{2}}{4 s} D^{f} \beta\left\{G_{1}(s)\left(1+\cos ^{2} \theta\right)+\left(1-\beta^{2}\right) G_{2}(s) \sin ^{2} \theta+2 \beta G_{3}(s) \cos \theta\right\} .
$$

The vector and axial vector coupling constants defined in Eq. 2, and the propagator in the lowest order Breit-Wigner approximation of the $Z$ resonance with mass $M_{Z}$ and width $\Gamma_{\%}^{0}$

$$
\chi_{0}(s)=R(s) \cdot K=\frac{s}{s-M_{\%}^{2}+i M_{\eta} \Gamma_{\%}^{0}} \cdot K
$$


with normalization

$$
K=\frac{G_{F} M_{Z}^{2}}{8 \sqrt{2} \pi \alpha}
$$

determine the functions in Eq. 8 as follows:

$$
\begin{aligned}
& G_{1}(s)=Q_{f}^{2}-2 v_{e} v_{f} Q_{f} \operatorname{Re} \chi_{0}(s)+\left(v_{e}^{2}+a_{e}^{2}\right)\left(v_{f}^{2}+\beta^{2} a_{f}^{2}\right)\left|\chi_{0}(s)\right|^{2} \\
& G_{2}(s)=Q_{f}^{2}-2 v_{e} v_{f} Q_{f} \operatorname{Re} \chi_{0}(s)+\left(v_{e}^{2}+a_{e}^{2}\right) v_{f}^{2}\left|\chi_{0}(s)\right|^{2} \\
& G_{3}(s)=-2 a_{e} a_{f} Q_{f} \operatorname{Re} \chi_{0}(s)+4 v_{e} a_{e} v_{f} a_{f}\left|\chi_{0}(s)\right|^{2} .
\end{aligned}
$$

Integrating over the solid angle, we obtain the total cross section for $e^{+} e^{-} \rightarrow f \bar{f}$ :

$$
\sigma_{i o t}=\sigma_{\gamma}+\sigma_{\gamma-Z}+\sigma_{Z}
$$

where $\sigma_{\gamma}$ is the familiar, pure electromagnetic cross section

$$
\sigma_{\gamma}=\frac{4 \pi Q_{f}^{2} \alpha^{2}}{3 s}\left[\frac{\beta\left(3-\beta^{2}\right)}{2}\right],
$$

$\sigma_{1-Z}$ is the interference term, and $\sigma_{Z}$ at $\sqrt{s}=M_{Z}$ is

$$
\sigma_{Z}=\frac{D^{f} G_{F}^{2} M_{Z}^{4}}{96 \pi \Gamma_{Z}^{2}}\left(v_{e}^{2}+a_{e}^{2}\right)\left[v_{f}^{2} \frac{\beta\left(3-\beta^{2}\right)}{2}+a_{f}^{2} \beta^{3}\right] \text {. }
$$

Three el ergy regions can be distinguished. In the low-energy region where $s \ll M_{2}^{2}$, we may neglect the terms arising from the effects of weak interactions and the cross section behaves as $1 / s$. In the intermediate-energy region, the $\mathcal{M}_{\gamma}-\mathcal{M}_{Z}$ interference term is no longer negligible, but $\left|\mathcal{M}_{Z}\right|^{2}$ is still tiny. This is the situation at PEP, PETRA, and TRISTAN with $E_{\mathrm{cm}}$ ranging from about $20 \mathrm{GeV} / c^{2}$ to $60 \mathrm{GeV} / c^{2}$. The effect of weak interactions in this energy region is to create measurable asymmetries in the decay angular distributions of pair-produced particles. A huge enhancement in the cross section occurs in the $Z$ resonance region where $s \approx M_{Z}^{2}$. As an example of this enhancement, cross sections for a possible fourth generation heavy down-type quark and heavy charged lepton are shown in Fig. 5 . In the range of $E_{\mathrm{cm}}$ between 89.2 and $93 . \mathrm{C} \mathrm{GeV}$ dominated by $Z$ decay, $\sigma_{\gamma}$ is smaller than $\sigma_{Z}$ by more than two orders of magnitude for the typical new particles being considered. Therefore, only decays of new particles through the $Z$ will be considered.

\subsubsection{Higher Order Corrections}

Careful attention must be paid to the effects of radiative corrections as they have substantial effects on the predicted physics of the $Z$. As will be outlined later, the expected 

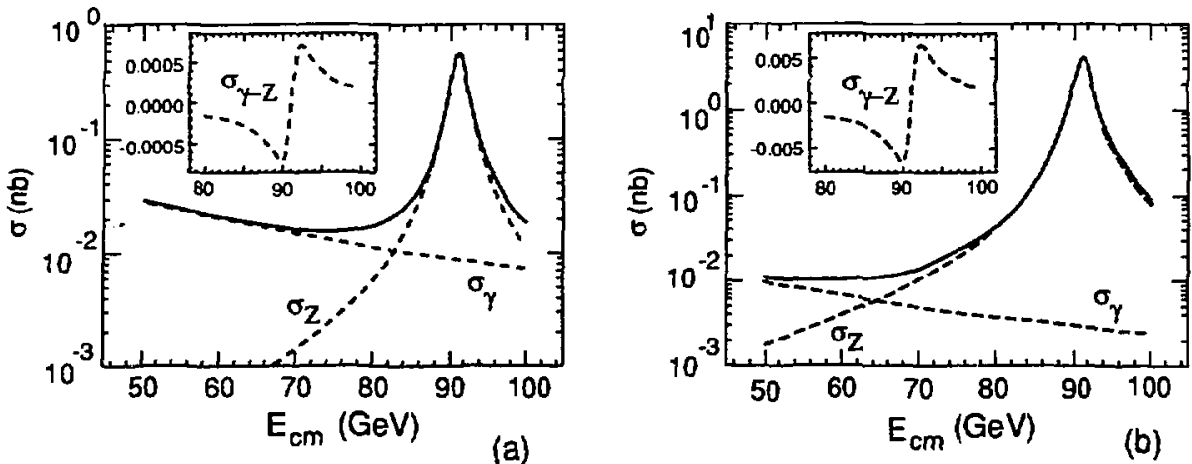

Figure 5: Tree-level cross section for a new (a) $35 \mathrm{GeV} / c^{2}$ charged heavy lepton; and (b) $35 \mathrm{GeV} / c^{2}$ fourth generation down-type quark $\left(b^{\prime}\right)$. Note the change in scales.

number of new quark and lepton events arising from $\boldsymbol{Z}$ decays will be normalized to the total number of hadronic $Z$ decays observed in our data sample. That is, we are less concerned with the accuracy of the absolute cross section scale over a range of $E_{\mathrm{cm}}$ than with the ratio of the $\mathcal{Z}$ hadronic partial width to the predicted new particle partial width. We will therefore concentrate on radiative corrections to $\Gamma_{Z}$. These corrections can be divided into tron classes: QCD and electroweak.

\section{QCD Corrections}

QCD corrections occur only in final states involving hadronic production with the $Z$ decaying into a quark-antiquark pair, $q \bar{q}$. The bulk of the correction is due to final state gluon radiation as shown in Fig. 6. QCD corrections to the width $\Gamma(Z \rightarrow q \bar{q})$ are known for non-zero quark masses up to first-order and for zero quark masses up to third-order in the strong coupling constant $\alpha_{s}$. Due to masses breaking chiral invariance and the large mass splitting between $t$ - and $b^{\prime}$-quarks, QCD corrections are different for vector and axial-vector couplings. Therefore, we first decompose the width given in Eq. 6 in the Born approximation into a vector and axial-vector part:

$$
\begin{aligned}
\Gamma^{0}(Z \rightarrow q \bar{q}) & =\frac{G_{F} M_{Z}^{3}}{2 A \sqrt{2} \pi} \beta \frac{\left(3-\beta^{2}\right)}{2} v_{q}^{2}+\frac{G_{F} M_{Z}^{3}}{24 \sqrt{2} \pi} \beta^{3} a_{q}^{2} \\
\Gamma_{q \bar{q}}^{0} & =\Gamma_{q \tilde{q}}^{0 V}+\Gamma_{q \bar{q}}^{0 A}
\end{aligned}
$$




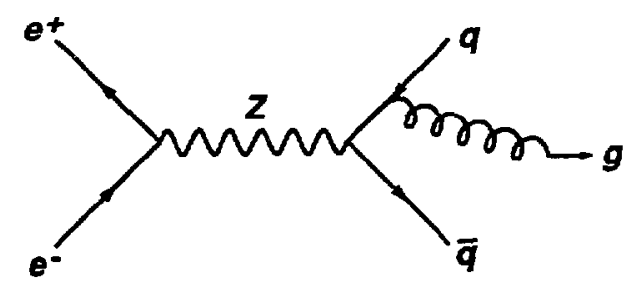

Figure 6: Feynman diagram describing final state gluon radiation in hadronic $Z$ decay.

The QCD corrections are then:

$$
\begin{aligned}
\Gamma_{q \bar{q}}= & \Gamma_{q \bar{q}}^{0 V}\left[1+c_{1}\left(\frac{\alpha_{s}}{\pi}\right)+c_{2}\left(\frac{\alpha_{s}}{\pi}\right)^{2}+c_{3}\left(\frac{\alpha_{s}}{\pi}\right)^{3}\right]+ \\
& \Gamma_{q \bar{q}}^{0 A}\left[1+d_{1}\left(\frac{c_{s}}{\pi}\right)+d_{2}\left(\frac{\alpha_{s}}{\pi}\right)^{2}+d_{3}\left(\frac{\alpha_{s}}{\pi}\right)^{3}\right]
\end{aligned}
$$

For a current determination of $\alpha_{\alpha}$, we refer the reader to Ref. [9] which indicates a value of the QCD scale parameter $\Lambda_{M S}^{(5)}=290 \pm 170 \mathrm{MeV}$ in the formula for the running coupling constant $[10]$ :

$$
\begin{aligned}
\alpha_{s}\left(n_{f}, \mu, \Lambda_{\overline{M S}}\right) & =\frac{1}{b_{0} \log \left(\mu^{2} / \Lambda^{2}\right)}-\frac{b_{1} \log \left(\log \left(\mu^{2} / \Lambda^{2}\right)\right)}{b_{0}\left(b_{0} \log \left(\mu^{2} / \Lambda^{2}\right)\right)^{2}} \\
b_{0} & =\frac{33-2 n_{f}}{12 \pi} \\
b_{1} & =\frac{153-19 \pi_{f}}{24 \pi^{2}},
\end{aligned}
$$

where $n_{f}$ is the number of quarks with mass less than the energy scale $\mu$ in the modified minimal subtraction (MS) renormalization scheme. We use $n_{f}=5$ and $\mu=M_{Z}$ (if we are assuming that the mass of the $t$-quark is less than $\left.M_{Z} / 2\right)$ resulting in a value of $\alpha_{s}=0.123 \pm 0.015$. If we assume $m_{t}<M_{Z} / 2$ in the case of searching for the $t$-quark, then $n_{f}=6$ is used.

Exact expressions for the first-order coefficients $c_{1}$ and $d_{1}$ in Eq. 16 have been calculated [11] and compact approximations [12] read:

$$
c_{1}=\frac{4 \pi}{3}\left[\frac{\pi}{2 \beta}-\frac{3+\beta}{4}\left(\frac{\pi}{2}-\frac{3}{4 \pi}\right)\right]
$$




$$
d_{1}=\frac{4 \pi}{3}\left[\frac{\pi}{2 \beta}-\left(\frac{19}{10}-\frac{22}{5} \beta+\frac{7}{2} \beta^{2}\right)\left(\frac{\pi}{2}-\frac{3}{4 \pi}\right)\right] .
$$

Note that for light quarks, the familiar result $\beta \rightarrow 1 ; c_{1}, d_{1} \rightarrow 1$ is reproduced. For massless quarks, first-order QCD corrections increase the hadronic width by $3.9 \%$ for $n_{f}=5$ and $\alpha_{s}=0.123$. It is only for the $b$-quark that the finite mass expressions above make a nonnegligible difference with $d_{1}=1.21$. However, for possible heavy new quarks, these massive quark QCD corrections are far more important as a consequence of the $1 / \beta$ singularity from Coulombic-gluon terms which predict a step function for the vector part of the width as $m_{q} \rightarrow M_{Z} / 2$, as will be discussed later.

In the $\overline{M S}$ renormalization scheme, the higher order coefficients for massless quarks are [13]:

$$
\begin{aligned}
& c_{2}=d_{2}=1.985-0.115 n_{f} \\
& c_{3} \approx d_{3}=70.98-1.2 n_{f}-0.005 n_{f}^{2}
\end{aligned}
$$

The sum of these second- and third-order corrections increase hadronic partial widths by only $0.6 \%$, and can be safely ignored since the uncertainty in $\alpha_{s}$ of 0.015 gives an uncertainty $0.4 \%$ in the hadronic partial widths after the first-order QCD correction.

\section{Electroweak Corrections}

Electroweak corrections include purely electromagnetic effects from final and initial state photon radiation and genuine electroweak or oblique [21] corrections from the dressing of propagators, along with box and vertex corrections.

Final state photon emission, as shown in Fig. 7, summed to all orders and partially cancelled by terms from final state vertex corrections constitutes a small correction of [14]:

$$
\Gamma_{f \bar{f}}=\Gamma_{f \bar{f}}^{0}\left(1+\frac{3 \alpha Q_{f}^{2}}{4 \pi}\right)
$$

This correction increases individual partial widths by at most $0.17 \%$ and can be ignored.

Initial state radiation substantially distorts the lowest-order Breit-Wigner $Z$ line shape. If an electron or positron radiates energy in the form of one or more photons before an interaction, the effective $E_{\mathrm{cm}}$ of the system decreases. Because of the resonance, the cross section is enhanced above the $Z$ pole (radiative tail), and is suppressed below the pole. These initial state radiative corrections do not affect the numerical value of $\Gamma_{Z}$ but rather affect how $\Gamma_{Z}$ is extracted from the measured resonance shape. 


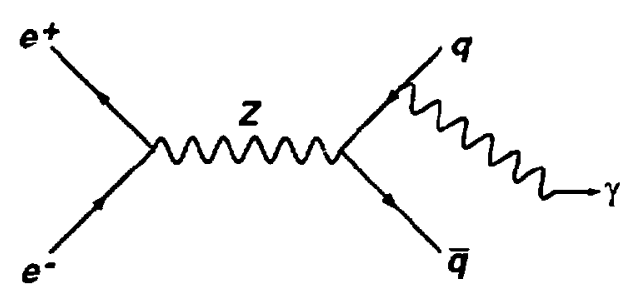

Figure 7: Feynman diagram describing final state photon radiation in $Z$ decay.

Genuine electroweak radiative corrections result from internal loops of leptons and bosons from the vacuum polarization of the photon and the self-energy of the $Z$ as shown in Fig. 8. Electroweak radiative corrections modify the Born relations and the effective values
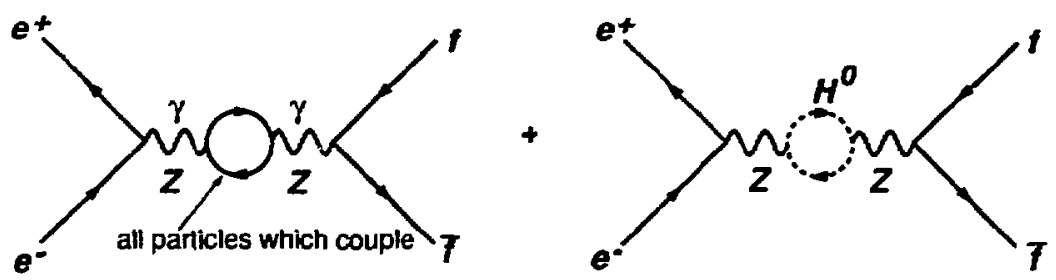

Figure 8: Feynman diagrams describing oblique or internal loop radiative corrections.

of the parameters of the Standard Model, such as $\sin ^{2} \theta_{W}$ and $\rho\left(\rho=\infty_{0}=M_{W}^{2} / M_{Z}^{2} \cos ^{2} \theta_{W}\right)$, whose values depend on the scale at which they are measured.

At tree level, the relation between $\sin ^{2} \theta_{W}$ and $M_{Z}$ is:

$$
\sin ^{2} \theta_{W} \cos ^{2} \theta_{W}=\frac{\pi \alpha}{\sqrt{2} G_{F} \rho_{0} M_{Z}^{2}}
$$

Beyond tree level,

$$
\sin ^{2} \theta_{W} \cos ^{2} \theta_{W^{r}}=\frac{\pi \alpha}{\sqrt{2} G_{F} \rho_{0} M_{Z}^{2}(1-\Delta r)}
$$




$$
\Delta r \equiv \Delta r\left(\alpha, \alpha_{s}, G_{F}, M_{Z}, m_{t}, m_{H}, \text { 'new' physics }\right)
$$

where $\Delta r$ embodies all of the $O(\alpha)$ radiative corrections [19] including the running of the electromagnetic coupling constant $\alpha$ up to the energy scale of the $Z$ [17]:

$$
\alpha\left(M_{2}^{2}\right)=\frac{\alpha}{1-\Delta \alpha}=1.064 \alpha \text {. }
$$

Virtual particles heavier than the $Z$ can circulate in the internal loops of Fig. 8; $\Delta r$ shows a strong dependence on $m_{t}$ and a weaker dependence on $m_{H^{\circ}}$. Heavier particles resulting from physics beyond the Standard Model can also contribute to $\Delta r$ in calculable amounts [15].

In the on-shell renormalization scheme [20], the simplest definition of $\theta_{W}$ is used in terms of the physical $W$ and $Z$ masses:

$$
\sin ^{2} \theta_{W}=1-\frac{M_{W}^{2}}{M_{Z}^{2}} \equiv s_{W}^{2} .
$$

Corrections to the calculations of partial widths from $\Delta r$ can be taken into account using an improved Born approximation [18] that includes the real parts of oblique corrections but ignores small corrections from imaginary parts of self-energies, vertices, and boxes. In all of the preceding expressions for $\Gamma_{Z}(Z \rightarrow f \bar{f})$, simply replace

$$
\begin{aligned}
G_{F} & \rightarrow \rho G_{F} \\
\rho & =\frac{1}{1-\Delta \rho}
\end{aligned}
$$

and in the calculation of the weak coupling constants, use an effective mixing angle:

$$
\bar{s}_{W}^{2}=s_{W}^{2}+c_{W}^{2} \Delta \rho
$$

That is,

$$
\begin{aligned}
& a_{f}=2 T_{3}^{f} \\
& v_{f}=2\left(T_{3}^{f}-2 Q_{j} \vec{s}_{W}^{2}\right) .
\end{aligned}
$$

The values of $s_{W}^{2}, \bar{s}_{W}^{2}$, and $\Delta \rho$ are obtained from the program SIN2TH which follows the explicit formulae for one-loop weak corrections in the on-shell scheme in Ref. [16] when calculating $\Delta r$. Note that $\bar{s}_{W}^{2}$ is equivalent to $s^{-2}\left(M_{Z}^{Z}\right)$ of Lynn and Kennedy $[21\}$, and $\left(\sin ^{2} \theta_{11}\right)_{\overline{11.5}}$ of Marciano and Sirlin [22]. 

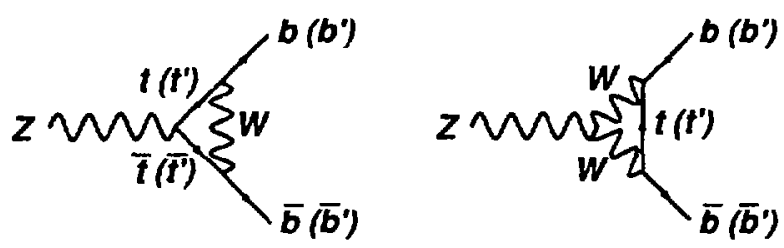

Figure 9: Feynman diagrams describing radiative corrections for the $Z-b \bar{b}$ (or $Z-b^{\prime} \bar{b}^{\prime}$ ) vertex.

For $f=b$ or $b$, there are additional large terms from the vertex corrections [23] of the type shown in Fig. 9. To include these terms, for $f=b, b^{\prime}$ only, we make the replacements:

$$
\begin{aligned}
\rho & \rightarrow \sqrt{\rho \rho_{b}} \\
\rho_{b} & =\rho\left(1-\frac{4}{3} \Delta \rho\right) \\
\bar{s}_{W}^{2} & \rightarrow \bar{s}_{W}^{2}\left(1+\frac{2}{3} \Delta \rho\right) .
\end{aligned}
$$

Including the $\Delta r$ and $Z-b \bar{b}$ electroweak correction terms changes the Born partial widths by up to $1.5 \%$ depending on the value chosen for the top quark mass.

\section{Numerical Results}

In the calculation of partial widths, the numerical values of $M_{Z}=91.14 \mathrm{GeV} / c^{2}$ [24], $m_{H}=100 \mathrm{GeV} / c^{2}$ and $\Lambda_{\overline{M S}}=290 \mathrm{MeV}$ (giving $\alpha_{s}\left(M_{Z}^{2}\right)=0.123$ ) are used. In all cases except for $m_{t}<M_{Z} / 2$, the value of $m_{t}=100 \mathrm{GeV} / c^{2}$ is chosen. From SIN2TH [16], for $m_{t}=100 \mathrm{GeV} / c^{2}$, the result $\Delta r=0.0575$ is obtained, and from the relation

$$
M_{W}^{2}\left(1-M_{W}^{2} / M_{Z}^{2}\right)=\frac{\pi \alpha}{\sqrt{2} G_{F}(1-\Delta r)}
$$

we get

$$
s_{W}^{2}=0.233 ; \bar{s}_{W}^{2}=0.230
$$

resulting in the partial widths listed in Table 5. For the case of $b$ and $\nu_{4}$, the values of $m_{t^{\prime}}=100 \mathrm{GeV} / c^{2}$ and $m_{L}=100 \mathrm{GeV} / c^{2}$ are chosen to keep the contribution [25] $\Delta r$ due 
Table 5: Partial widths of $Z$ to the known fundamental fermions.

\begin{tabular}{lc}
\hline$f$ & $\begin{array}{c}\text { Partial Width } \\
\Gamma(Z \rightarrow f \bar{f}) \\
(\mathrm{GeV})\end{array}$ \\
\hline$\nu_{t}, \nu_{\mu}, \nu_{\tau}$ & 0.166 \\
$e^{-}, \mu^{-}, \tau^{-}$ & 0.0835 \\
$u, c$ & 0.296 \\
$d, s$ & 0.381 \\
$b$ & 0.376 \\
Hadronic (udscb) & 1.73 \\
Total & 2.48 \\
\hline \hline
\end{tabular}

to the presence of new fermion generation $\left(\begin{array}{c}f_{+} \\ f_{-}\end{array}\right)$:

$$
\Delta r_{\text {new }}=\frac{\alpha}{4 \pi} \cdot \frac{3 \sin ^{2} \theta_{W}}{4 \cos ^{2} \theta_{W}} \cdot \frac{m_{+}^{2}-m_{-}^{2}}{M_{W}^{2}}
$$

down to an absolute value less than 0.0002 .

The partial widthe for the $Z$ decaying into new sequential quarks and leptons as a function of mass are shown in Fig. 10.

\section{Uncertainties in Heavy Quark Partial Widths}

The partial widths for $t$ - and $b^{\prime}$-quarks are subject to uncertainties due to an insufficient knowledge of higher order QCD corrections for massive quarks. It is anticipated that the potentially large higher order corrections might sum up to modify the leading correction term by only a factor $\left(1-\exp \left(-2 \pi \alpha_{s} / 3 \beta\right)\right)$ similar to the result in $Q E D$, and uncertainties are estimated [32] to be $\pm 30 \%$ of the first order QCD correction as shown in Fig. 11.

The uncalculated higher order corrections are expected to alter the $O\left(\alpha_{s}\right)$ result significantly in the region where the first order result exceds the Born tern close to threshold ( $\left.m_{Q} \approx E_{r m} / 2\right)$ and perturbative $Q C D$ breaks down. In this mass range, the produced quarks see a strong force potential, briefly form a bound state, and exchange coulombic gluons resulting in an increased partial width. For $s$ far larger than $4 m_{0}^{2}$ the difference between energy and momentum for the scale $\mu$ of $\alpha_{2}\left(\mu^{2}\right)$ is unimportant. When approaching the threshold region, the choice $\mu^{2}=4 p_{t}^{2}$ where $p_{t}=\beta M_{\%} / 2$ mimics the onset of the 


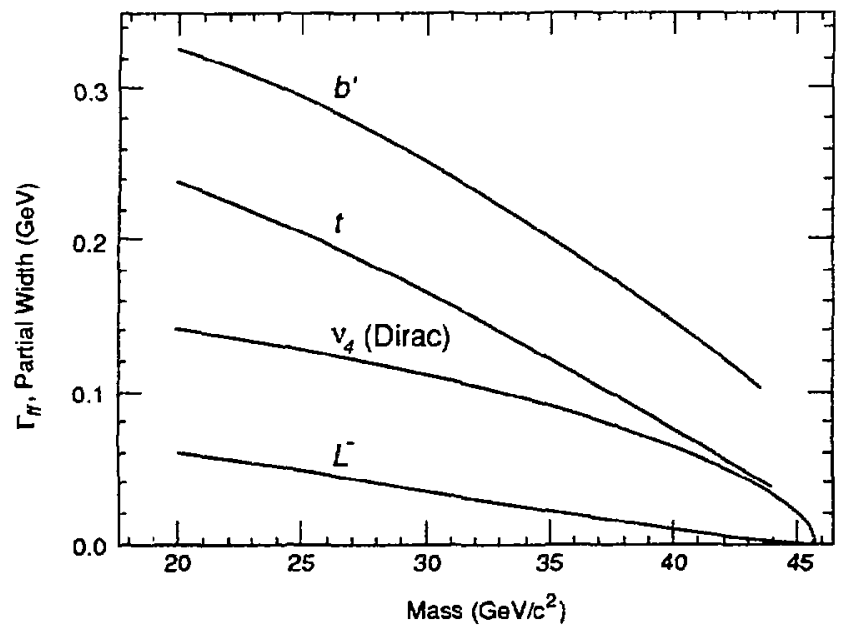

Figure 10: Partial widths for new heavy fermions as a function of mass.

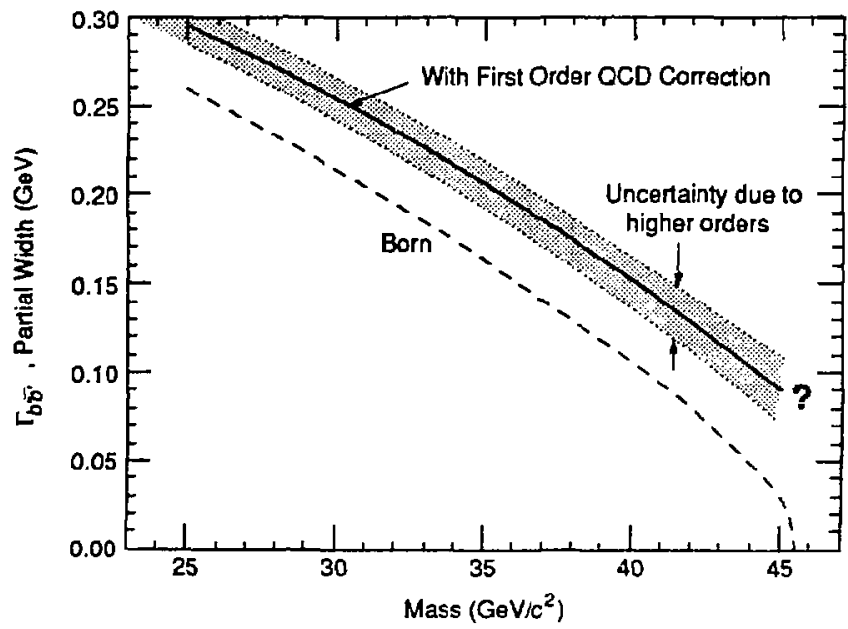

Figure 11: Uncertainty in heavy quark $\left(b^{\prime}\right)$ partial width due to uncertainty in higher order QCD corrections. The question mark indicates the region where perturbative QCD breaks down 
nonperturbative behavior [32]. Different treatments of the calculation of partial widths in this region [26] disagree by as much as a factor of two. The typical mass $m_{Q_{4}}$ of a possible heavy quark ( $t$ or $b^{\prime}$ ) hadron is estimated to be $m_{Q q} \simeq m_{Q}+400 \mathrm{MeV}$, and to avoid controversial treatments and to be conservative, the Born partial width is used in all subsequent calculations in the mass range close to threshold defined as $\left(E_{\mathrm{cm}} / 2-600 \mathrm{MeV}\right)$ $<m_{Q}<E_{\mathrm{cm}} / 2$. The Born partial width is an underestimation of the partial width to all orders.

\subsection{The Top Quark $(t)$}

While the $t$-quark has not yet been found, its existence is supported by the measured properties of the $b$-quark. In the framework of the Standard Model, the $t$-quark decays vis a virtual $W$ boson $\left(W^{*}\right)$ in a charged current (CC) process into a $b$-quark. The decay $t \rightarrow b H^{+}$ would dominate the standard charged current if a light enough charged Higgs component of an extended scalar sector with several Higgs doublets exists. If the $H^{ \pm}$decays hadronically, then the standard search strategies at $p \vec{p}$ colliders, looking for hard isolated leptons, would not be sensitive to such a possibility. Much of the following discussion can also be applied to any other hesvy quark such as a fourth generation $b^{\prime}$ with mass less than $M_{Z} / 2$.

\subsubsection{Why the t-quark Must Exist}

There is much indirect evidence for the existence of the $t$-quark It is an essential part of the third generation of $S U(2)$ doublets and singlets:

$$
\left(\begin{array}{c}
\nu_{\tau} \\
\tau
\end{array}\right)_{L}\left(\begin{array}{l}
t \\
b
\end{array}\right)_{L}\left(\begin{array}{lll}
(\tau)_{R} & (t)_{R} & (b)_{R} .
\end{array}\right.
$$

A non-zero forward-backward asymmetry measurement of tagged b-jets in $e^{+} e^{-} \rightarrow b \bar{b}$ at PEP, PETRA, and TRISTAN [27] indicates that the axial coupling of the b-quark to $Z$ is non-zero, so the b-quark is in a doublet and there has to be a heavier quark to be its partner. The heavier quark is, by definition, the $t$-quark. In addition, if $b_{L}$ were a singlet like $b_{R}$, then flavor-changing neutral-current decays of $B$ mesons would result [28]. An upper limit [29] of $B r\left(B \rightarrow \ell^{+} \ell^{-} X\right)<0.0012$ again shows that $b_{l}$ is in a doublet. Finally, cancellation of triangle chiral anomalies [30], which is crucial to the renormalizability of the electroweak theory, requires the same number of generations of quarks as of leptons; therefore, the existence of the $\nu_{r}-\tau$ generation requires the existence of a $t-b$ generation. 


\subsubsection{Heavy Quark Fragmertation}

Fragmentation is the process describing the organization of colored quarks into colorless hadrons involving the creation of additional quark-antiquark pairs by the color field as shown in Fig. 12. It is generally anticipated [31] that almost all of a heavy (i.e. mass

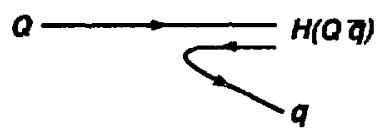

Figure 12: Fragmentation of heavy quark $Q$ to heavy hadron $H(Q \bar{q})$. The quark half of the $q \bar{q}$ pair is free to carry on the fragmentation process, continuing until there is insufficient energy to produce new $q \bar{q}$ pairs.

greater than $m_{b}$ ) quark's original energy will reside in a meson or baryon carrying the heavy quark $Q$ after fragmentation. From energy density arguments [32], $Z$ bosons are expected to decay into a pair of $Q$ hadrons and at most a few pions of very low energy ( $\sim 1 \mathrm{GeV}$ ).

Perturbative QCD cannot be used to calculate fragmentation behavior and serai-empirical methods are needed to describe it. The framentation function $f(z)$ is a parameterization of the fraction of energy and momentum parallel to the parent quark direction $\left(p_{\|}\right)$carried away by the produced hadron with

$$
z \equiv \frac{\left(E+p_{\|}\right)_{\text {hadron }}}{\left(E+p_{\|}\right)_{\text {quark }}}
$$

Fragmentation functions considered are the Peterson model [33]

$$
f(z)=\frac{1}{z\left(1-\frac{1}{z}-\frac{c}{1-z}\right)^{2}}
$$

where $\epsilon=\left(m_{0} / m_{Q}\right)^{2}$ with $m_{0}$ some reference scale and $m_{Q}$ the heavy quark mass; and the Lund Symmetric model [34]:

$$
f(z)=\frac{1}{z}(1-z)^{a} \exp \left(-b m_{T}^{2} / z\right),
$$

where $m_{T}$ is the transverse mass of the produced hadron and $a=0.45$ and $b=0.9 \mathrm{GeV}^{-2}$ are parameters chosen to fit experinental distributions. 


\subsection{3 t-quark Charged Current Decays}

The charged current decay of hadrons containing a $t$-quark in the spectator model is considered as shown in Fig. 13(a) where the light $\bar{q}$-quark acts as a spectator and plays
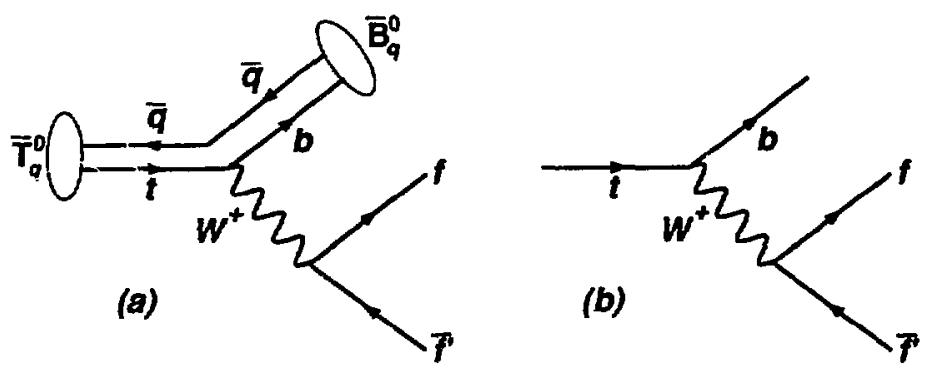

Figure 13: (a) Decay of a top hadron in the spectator model; (b) effective Feynman diagram for decay of a top quark.

no role in the decay. This model should be particularly valid for any heavy new quark constituting a hadron, and the simpler Feynman diagram of Fig. 13(b) conveys the same information. The $t$-quark decays primarily to a $b$-quark and a virtual $W$ with a rate:

$$
\Gamma\left(t \rightarrow b W^{*}\right)=9 \frac{G_{F}^{2} m_{t}^{5}}{192 \pi^{3}}\left|V_{t b}\right|^{2} f\left(\frac{m_{t}^{2}}{M_{W}^{2}}, \frac{m_{b}^{2}}{m_{t}^{2}}\right),
$$

where $\left|V_{t b}\right|^{2} \simeq 1$ and $f(\rho, \mu)$, given in Ref. [35], is a function that needs to be numerically integrated to explicitly take the $W$-propagator and non-zero $b^{\prime}$-quark mass into account, but which approaches unity for $m_{1} \gg m_{b}$ and $m_{t} \ll M_{W}$. A fraction $2 / 3$ of $t$-quarks with $m_{t} \gg m_{c}$ will decey into three jets $t \rightarrow b_{u} \bar{d}$ and $b c \bar{s}$, and $1 / 9$ to $t \rightarrow b e^{+} \nu_{e}, \mu$, and $\tau$ each. We are interested in the semileptonic decays which can result in an isolated lepton with both high momentum and high transverse momentum with respect to the associated quark jet, giving a distinctive signature. The branching ratio for semileptonic decay is modified slightly by QCD corrections [36]:

$$
\operatorname{Br}\left(t \rightarrow b e^{+} \nu_{e}\right)=\frac{1}{3+6\left(1+\alpha_{s} / \pi\right)}
$$

owing to virtual gluon exchange and emission in the light quark decay modes. 
If the $W^{*}$ decays hadronically, then spherical events result, which can be characterized by certain event shape parameters. The presence of a $t$-quark can then be checked using the two different topologies.

\subsection{4 t-quark Decays into a Charged Higgs}

Looking beyond the Standard Model, whe are led to consider an extended scalar sector with more that one Higgs doublet [37]. If the charged Higgs components of these doublets are not too heavy, the decay $t \rightarrow b H^{+}$as shown in Fig. 14 will dominate standard charged

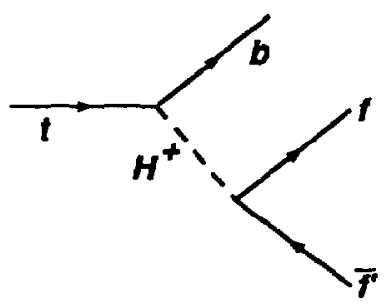

Figure 14: Feynman diagram describing decay of $t$ through a real charged Higgs $H^{+}$.

current decays. The $H^{+}$would decay dominantly via $H^{+} \rightarrow c \bar{s}$ and $H^{4} \rightarrow \tau \nu$ modes resulting in signatures making their detection at $p \bar{p}$ colliders dificult, even suggesting [38] that the existing mass limits on $m_{t}$ from $p \bar{p}$ colliders may not be valid if a light $H^{+}$exists.

In the two-Higga-doublet (THD) models, one doublet $\phi_{1}$ gives mass to $T_{3}=-1 / 2$ quarks and the other doublet $\phi_{2}$ gives mass to $T_{3}=1 / 2$ quarks vis vacuum expectation values $v_{1}$ and $v_{2}$ where

$$
v_{1}^{2}+v_{2}^{2}=\frac{s}{\sqrt{2} G_{F}} .
$$

The THD model leads to five physical Higgs bocons: two neutral scalars (CP even) $H_{1}^{0}$ and $H_{2}^{0}$, one neutral pseudoscalar ( $C P$ odd) $H_{p}^{0}$, and two charged scalars $H^{+}$and $H^{-}$. All the masses and the ratio $\tan \beta_{m i x}=v_{1} / v_{2}$ are a priori unknown. The present mass limit of $m_{H^{+}}>19 \mathrm{GeV} / c^{2}$ at $95 \% \mathrm{CL}$ has been determined by CELLO [39] for the charged scalars. 
If $m_{t}>m_{H^{+}}+m_{b}$, then the on-shell decay width of $t \rightarrow b H^{+}$is [38]:

$$
\begin{array}{r}
\Gamma\left(t \rightarrow b H^{+}\right)=\frac{\sqrt{2} G_{F}}{8 \pi m_{t}^{3}}\left[m_{t}^{2} \cot ^{2} \beta_{m i x}+m_{b}^{2} \tan ^{2} \beta_{m i x}\right] \cdot\left(m_{t}^{2}+m_{b}^{2}-m_{H^{+}}^{2}+2 m_{b} m_{i}\right) \\
\times\left(m_{i}^{4}+m_{b}^{4}+m_{H^{+}}^{4}-2 m_{b}^{2} m_{t}^{2}-2 m_{H^{+}}^{2} m_{t}^{2}-2 m_{b}^{2} m_{H^{+}}^{2}\right)^{1 / 2} .
\end{array}
$$

The term in square brackets depends on $\beta_{m i x}$ and has a minimum of $2 m_{b} / m_{t}$. Note that $\Gamma\left(t \rightarrow b H^{+}\right) \propto G_{F} m_{t}^{3}$ as a two-body decay, while the charged current jecay width $\Gamma\left(t \rightarrow b f \bar{f}^{\prime}\right) \propto G_{F}^{2} m_{t}^{5}$ as a three-body decay. As an example, assuming $m_{t}=40 \mathrm{GeV} / c^{2}$ and $m_{H^{+}}=25 \mathrm{GeV} / c^{2}$, the minimum value of $\Gamma\left(t \rightarrow b H^{+}\right)$is $3.0 \times 10^{-3} \mathrm{GeV}$ to be compared with $\Gamma\left(t \rightarrow b W^{*}\right)=2.1 \times 10^{-5}$. In this typical case to be considered, the decay width of the $t$ into a real $H^{+}$is at least a factor of 100 times larger than the charged current decay. The $\mathrm{H}^{+}$couples preferentially to the heaviest available fermions, and branching fractions depend on the value of $\beta_{m i x}$. These branching fractions can be estimated as [40]:

$$
B r\left(H^{+} \rightarrow \tau^{+} \nu\right) \simeq 1 /\left(1+3 \tan ^{4} \beta_{m i x}\right) ; \quad B r\left(H^{+} \rightarrow c \bar{s}\right) \simeq 1 /\left(1+\frac{1}{3} \cot ^{4} \beta_{m i x}\right)
$$

In the following searches for $t \rightarrow b H^{+}$, arbitrary mixtures of $\mathrm{H}^{+} \rightarrow \mathrm{cj}$ and $\mathrm{H}^{+} \rightarrow \gamma^{-}$will be considered, and if $m_{t}>m_{b}+m_{H^{+}}$, then it will be assumed that the $t$-quark decays $100 \%$ through a real charged Higgs. The topology of these decays will in general also produce spherical events and large momentum sums out of the event plane.

\subsubsection{Present $t$-quark Mass Limits}

The reaction $e^{+} e^{-} \rightarrow q \bar{q}$ is a model independent way to search for new heavy quarks. Unambiguous limits come from studies at TRISTAN [41] giving $m_{t}>27.7 \mathrm{GeV}$ at the $95 \%$ confidence level (CL). More model-dependent and somewhat less direct $t$-quark searches rely on signatures in hadronic reactions in $p \bar{p}$ collisions from $W$ decays to $t \bar{b}$ or via quarkantiquark $(q \bar{q} \rightarrow t \bar{t})$ and gluon-gluon fusion $(g g \rightarrow t \bar{t})$. The limit from UA1 [42] is $m_{t}>44$ $\mathrm{GeV} / c^{2}$ at $95 \% \mathrm{CL}$; from CDF [43], $m_{t}$ is excluded between 40 and $77 \mathrm{GeV} / c^{2}$ at $95 \%$ $\mathrm{CL}$; and $m_{t}>67 \mathrm{GeV} / c^{2}$ from UA2 [44]. It is stressed that all of these $p \bar{p}$ collider limits assume $100 \% \mathrm{CC}$ decays of the $t$-quark. An effort has been made [45] to reinterpret the UA1 data to place a limit on $t$ decaying only through a charged Higgs, but it assumes a large branching fraction for $H \rightarrow \tau \nu_{\tau}$.

From theoretical considerations [46] of the ARGUS and CLEO measurements of $B \bar{B}$ mixing [4], $m_{l}$ should be greater than about $50 \mathrm{GeV} / c^{2}$. 
An upper bound on $m_{t}$ can be determined from comparison of experimental data to theoretical predictions with radiative corrections. Electroweak radiative corrections depend on $m_{i}$ and $m_{H^{\circ}}$ because $t$ and $H^{0}$ appear in virtual loops as described earlier. Consistency of world electroweak data with a common set of Standard Model parameters place bounds on $m_{t}$. Several comprehensive analyses [47] broadly agree:

$$
\begin{array}{ccc}
m_{t}<200(180) \mathrm{GeV} / c^{2} & \text { if } & m_{H^{0}}<1000(100) \mathrm{GeV} / c^{2} \\
m_{t}<168 \mathrm{GeV} / c^{2} & \text { if } & m_{H^{0}}<M_{Z}
\end{array}
$$

It can be seen that it is difficult to accommodate $m_{t}<M_{Z} / 2$ in the three generation Standard Model; however, experimental measuremerts leading to unambiguous mass limits are always desirable. In particular, decaj is of the $t$-quark through $H^{+}$can also be unambiguously excluded in $e^{+} e^{-}$collisions.

\subsection{Fourth Generation $Q=-1 / 3$ Quark $\left(b^{\prime}\right)$}

The possibility exists that a fourth generation weak isospin $-1 / 2$, charge $-1 / 3$ quark, usually known as the $b^{\prime}$-quark, has a mass $m_{b^{\prime}}<M_{\bar{Z}} / 2$. The part of the cross section which is induced through the neutral vector current is nearly a factor of four larger than the corresponding one for $t$, and a relatively large branching ratio for $Z \rightarrow b^{\prime} \bar{b}^{\prime}$ is expected as shown in Fig. 10. If $m_{b^{\prime}}>m_{t}$, the charged current decay $b^{\prime} \rightarrow t W^{*}$ is expected to dominate, but then the $t$-quark as described in the previous section would also be pair-produced and detected. We therefore only consider the case $m_{b^{\prime}}<m_{t}$.

\subsection{1 $\quad b^{\prime}$-Quark Decay Modus}

If $m_{b^{\prime}}<m_{t}$, then, as shown in Fig. 15(a), the $b^{\prime}$-quark will undergo the charged current decay $b^{\prime} \rightarrow c W^{*}$ which is suppressed by the mixing matrix element $V_{c b^{\prime}}$ that is expected to be small since it is a transition across two generations. We aiso assume $m_{t^{\prime}}>m_{b^{\prime}}$ following the pattern $m_{c}>m_{s}$ and $m_{t}>m_{b}$. From an extension of the Wolfenstein parameterization [48] of the KM matrix to four generations [49], the estimate

$$
\left|V_{c b^{\prime}}\right| \simeq \frac{1}{2} \theta_{c}^{4} \text { or } \frac{1}{2} \theta_{c}^{5}
$$

can be made where $\sin \theta_{c} \simeq 0.23$ is the Cabibbo angle. A significant reduction in the CC decay rate is expected, and depending on the mass assignment for $t$-quark and $t^{\prime}$-quarks, 
and the choice of urknown mixing angles, induced flavor-changing neutral-currents (FCNC) decays shown in Fig. 15(b) could compete or might even dominate the CC decay. FCNC
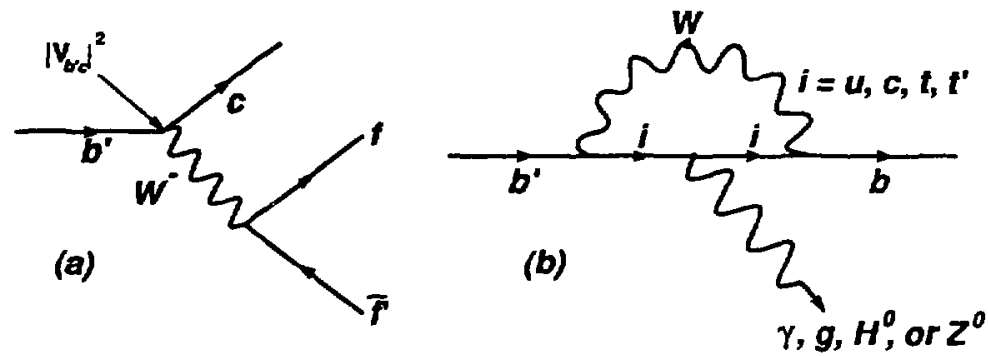

Figure 15: (a) Charged current (CC) and (b) Alavor-changing neutral-current (FCNC) decays of the $b^{\prime}$-quark.

decays are enbanced since the relevant mixing matrix elements $V_{t b^{\prime}} V_{t b}^{*}$ and $V_{t^{\prime} b^{\prime}} V_{t^{\prime} b}^{*}$ are less suppressed than $V_{c b^{\prime}}$, and also because the loop amplitude grows with the mass of the virtual quarks $\left(t\right.$ or $t^{\prime}$ ) in the loop. FCNC decays will dominate [50] if

$$
\frac{\left|V_{c b^{\prime}}\right|}{\left|V_{b t^{\prime}}\right|\left|V_{b^{\prime} t^{\prime}}\right|}<10^{-2}
$$

The relative fractions of FCNC hadronic and photonic decays roughly follows the ratio of $\alpha_{s}$ to $\alpha$, but also shows a complicated dependence on $m_{t}$ and $m_{t^{\prime}}$.

For our case of $m_{b}<M_{Z}+m_{b}$, off-shell $Z$ contributions are an order of magnitude or more below $b^{\prime} \rightarrow b \gamma$ transitions, and are not considered in the following studies. Under these assumptions the distinctive FCNC modes [51]

$$
b^{\prime} \rightarrow b \text { gluon and } b^{\prime} \rightarrow b \gamma
$$

could become dominant, with the first channel leading to $b^{\prime} \rightarrow b+$ hadrons and four jet events, and the second channel to events with isolated high energy photons. Since there are so many unknowns, arbitrary mixtures of CC and FCNC decays are considered as well as arbitrary fractions of $b^{\prime} \rightarrow b g$ and $b^{\prime} \rightarrow$ or in the FCNC part. If $m_{H^{0}}+m_{b}<m_{b^{\prime}}$, the decay $b^{\prime} \rightarrow b H^{0}$ can be the dominant FCNC mode [52]. If the $H^{0}$ is heavier than about 10 $\mathrm{GeV} / c^{2}$, it decays primarily into $b \bar{b}$, resulting in a six jet final state. We also search for the case $b^{\prime} \rightarrow c H^{-}$and the detection efficiency for $b^{\prime} \rightarrow b H^{0}$ is expected to be similar. Any 
niass limit obtained for $b^{\prime} \rightarrow c H^{-}$can be applied to $b^{\prime} \rightarrow b H^{0}$ if $m_{H /}$ is replaced by $m_{1 / l^{0}}$; therefore, we do not directly consider the decay through a neutral Higgs boson.

Small, suppressed decay widths translate into long lifetimes and the concern that decays may not occur within the detector volume, or else affect track trigger efficiencies and track selection efficiencies. From sensible extensions of the KM matrix to four generations such as in Eq. 43, lifetimes of only up to $10^{-12}$ seconds are anticipated. Decay vertices may then be observable in vertex drift chambers, but would only negligibly affect triggering or track selection efficiencies. Searches for long-lived $b^{\prime}$-quarks such as performed by the UA1 Collaboration [53] are not included in this work.

Finally, if a $H^{+}$charged scalar exists vith $m_{b^{\prime}}>m_{c}+m_{H^{+}}$, then the decay $b^{\prime} \rightarrow c H^{+}$ will dominate both the FCNC and CC decays just as in the $t$-quark case.

\subsubsection{Present $b^{\prime}$-quark Mass Limits}

Comprehensive searches for both CC and FCNC decays of the $b^{\prime}$-quark have been performed at TRISTAN [54] resulting in the limits $m_{b^{\prime}}>28.4 \mathrm{GeV} / c^{2}$ (CC decay) and $m_{:} \cdot>28.3 \mathrm{GeV} / c^{2}\left(100 \%\right.$ FCNC decays, with arbitrary mixtures of $b^{\prime} \rightarrow b \mathrm{~g}$ and $\left.b^{\prime} \rightarrow b \gamma\right)$. Again, the $p \bar{p}$ colliders give more model-dependent limits which consider CC decays: UA1 finds [42] $m_{b^{\prime}}>32 \mathrm{GeV} / c^{2}$, and UA2 gives [44] $m_{b^{\prime}}>53 \mathrm{GeV} / c^{2}$ at $95 \% \mathrm{CL}$.

Limits on deviations of measured electroweak parameters from theoretical predictions using radiative corrections which gave an upper bound on $m_{t}$ can be extended to new generations, but only give an upper bound on the mass splittings between members of isospin doublets:

$$
\begin{aligned}
\left(m_{t^{\prime}}-m_{b^{\prime}}\right)^{2}+\frac{1}{3}\left(m_{\nu_{4}}-m_{L}\right)^{2}+\left(m_{t}-m_{b}\right)^{2}< & \left(195 \mathrm{GeV} / c^{2}\right)^{2} \\
& \text { (with } \left.m_{H^{0}}=1000 \mathrm{GeV} / c^{2}\right) .
\end{aligned}
$$

\subsection{Heavy Neutral Lepton (Neutrino, $\nu_{4}$ )}

The pattern of masses within generations of the known fundamental fermions suggests that the lightest member of a new, fourth generation should be its neutrino. Since neutrinos have no electric charge and only weak charge, s-channel pair-production can only occur through a real or virtial $Z$ in $e^{+} e^{-}$annihilation. A dramatic increase in the production rate therefore occurs at the $Z$ resonance in contrast to lower $e^{+} e^{-}$annihilation energies where most processes occur through a virtual $\gamma$. With $6 \%$ of $Z$ decays going into $\nu \bar{\nu}$ for 
each light neutrino in a weak doublet, additional neutrinos are very amenable to detection at the SLC.

\subsubsection{Neutrino Mass and Mixing}

In the Electroweak $S U(2)_{L} \otimes U(1)_{Y}$ model, the photon and all three species of ncutrinos have zero mass. For the photon, masslessness is a natural consequence of exact electromagnetic guage invariance; its validity being well verified experimentally by the present bound $m_{r}<6 \times 10^{-16} \mathrm{eV}$. However, the masslessness of neutrinos is not on such firm theoretical or experimental footing. Theoretically, $m_{\nu}=0$ because only the left-handed component $\nu_{L}$ of each neutrino species is employed (the right-handed component $\nu_{R}$ is assumed not to exist) and lepton number conservation is required. Relaxing either of these constraints can lead to $m_{\nu} \neq 0$. Indeed, the present bounds [55]

$$
\begin{aligned}
& m_{\nu_{e}}<20 \mathrm{eV}(95 \% \mathrm{CL}) \\
& m_{\nu_{\mu}}<0.25 \mathrm{MeV}(90 \% \mathrm{CL}) \\
& \pi \nu_{\nu_{\tau}}<35 \mathrm{MeV}(95 \% \mathrm{CL})
\end{aligned}
$$

leave considerable room for speculation that neutrinos actually do possess mass. From the observation that particles of successive generations have higher masses, a possible fourth generation neutrino may have a mass considerably larger than the above limits. Indeed, many theories [58] assert the existence of one or more beavy neutral leptons, many with masses below $M_{Z} / 2$. Heavy neutrinos are also the original weakly-interacting-massiveparticle (WIMP) contenders for cold dark matter postulated for the closure of the universe [59].

According to the Standard Model, lepton masses come about tbrough a Yulkawa-like coupling of the lepton felds to the vacuum expectation value of the Higgs field. The fermion masses generated by the Higgs mechanism are totally arbitrary, their values are chosen to agree with experiment. If right-handed components exist, then neutrinos can also be given arbitrary masses by the Higgs mechanism.

For an additional fourth generation neutrino with mass, the weak and mass eigenstates do not necessarily coincide, just as for the quark sector. This can be conveniently expressed in terms of a unitary mixing matrix $U$ which "rotates" the neutrino mass eigenstates $\nu_{i}$ 
$(i=1,4)$ to the weak eigenstates $\nu_{\varepsilon}(\ell=e, \mu, \tau$, and $L)$, so that

$$
\left|\nu_{\ell}\right\rangle=\sum_{i=1}^{4} U_{\ell i}\left|\nu_{i}\right\rangle .
$$

There are no flavor-changing neutral currents (FCNC) because of the GMM mechanism [61] in this scenario since all the neutral leptons have the same value of weak isospin. Using the above notation, the particle of interest is the mass eigenstate $\nu_{4}$. Theory provides little guidance for a choice of mixing scenarios, though a reasonable assumption is preferential mixing to the closest generation such that $\left|U_{e 4}\right|<\left|U_{\mu 4}\right|<\left|U_{T 4}\right|$.

\subsubsection{Dirac and Majorana Type Neutrinos}

Particles with electric charge are clearly distinct from their antiparticles by their electromagnetic properties, but it is not obvious in what way elementary neutral particles should differ from their antiparticles. A Majorana particle [56] is one which is identical to its antiparticle, while a Dirac particle is one which is distinct from its antiparticle. A massive Dirac neutrino consists of the four states $\left(\nu_{-}^{D}, \bar{\nu}_{+}^{D}\right)$ and $\left(\bar{\nu}_{-}^{D}, \nu_{+}^{D}\right)$ where the subscripts indicate negative and positive helicities. A Majorana neutrino consists only of the two states $\left(\nu_{-}^{M}, \nu_{+}^{M}\right)$. For massless neutrinos, the distinction makes no difference, since the standard weak interactions couple only to left-handed states. States may be physically distinct because of their helicities, whether or not $\nu=\bar{\nu}$.

The masses of the neutrinos of the presently known three generations have been constrained to be small, but experimental results allow the neutrinos to be of either Dirac or Majorana type. New neutrinos could have large masses and be of either type. In particular, the widely regarded "see-saw mechanism" models [57], which attempt to explain the small masses of the neutrinos of the first three generations, predict the presence of both Majorana and Dirac type new heavy neutrinos.

\subsubsection{Neutrino Partial Widths}

The partial width for the $Z$ decaying into a sequential fourth generation Dirac neutrinoantineutrino pair (i.e. distinct fermions) is simply obtained by substituting the appropriate values of the weak coupling constants $v_{\nu}=a_{\nu}=1$ into the expression for partial widths given in Eq. 5.

When the $Z$ decays into a peir of sequential Majorana neutrinos, they coherently interfere with each other since they are identical fermions. The production amplitude through 
$Z$ decay therefore has to be antisymmetrized and integrated over only half the phase space [60]. The Majorana neutrino vector coupling then cancels out for any combication of ieftand right-handed couplings, and the axial-vector coupling is doubled. At tree level, this results in:

$$
\Gamma(Z \rightarrow \nu \bar{\nu})=\frac{G_{F}^{2} M_{Z}^{3}}{12 \pi \sqrt{2}} \times \begin{cases}\beta\left(3+\beta^{2}\right) / 4 & \text { Dirac; } \\ \beta^{3} & \text { Majorana, }\end{cases}
$$

where $\beta=\left(1-4 m_{\nu}^{2} / s\right)^{1 / 2}$. These two widths are compared in Fig. 16. Note that the widths for the two types of neutrinos are identical at zero mass where the distinction between the two types vanishes.

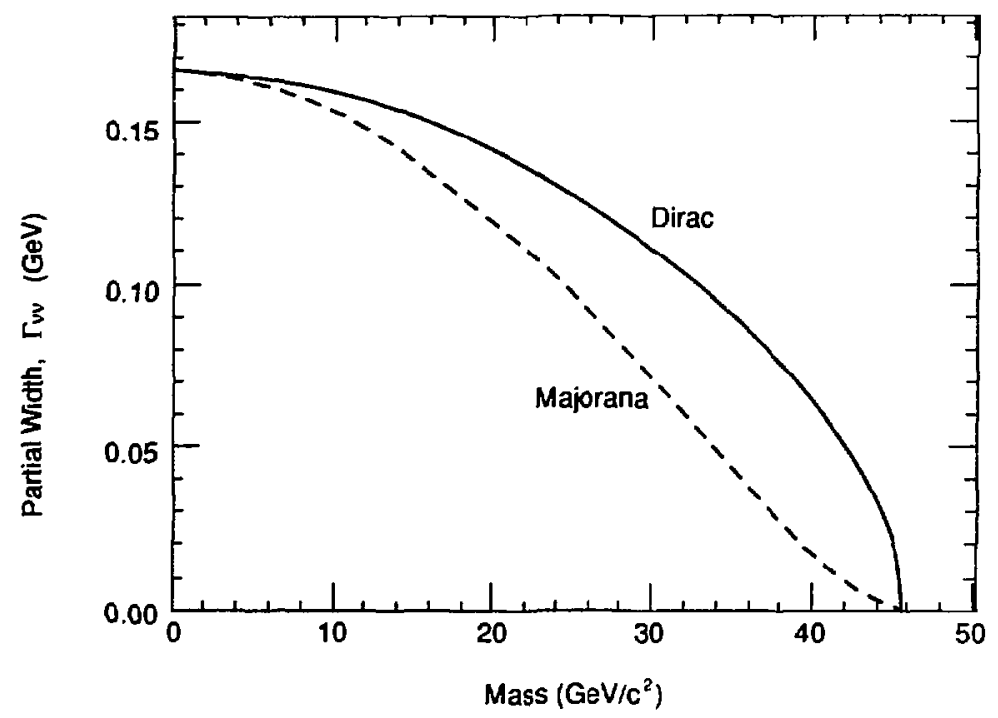

Figure 16: Partial widths for $Z$ decay into Dirac (solid line) and Majorana (dashed line) neutrinos.

Massive neutrinos can also be produced through other channels in $e^{+} e^{-}$annihilation. A massive neutrino can exist in a $S U(2)_{L}$ singlet, as predicted by some theories [62], and we will denote these neutrinos by $N$. The processes $e^{+} e^{-} \rightarrow N \bar{N}$ and $e^{+} e^{-} \rightarrow \bar{\nu}_{R} N$ are possible through $t$-channel $W$ exchange. However, the $W$ exchange proceeds by the matrix element 
$U_{W}$ in the lepton sector, analogous to the quark sector, and the two previous production rates are suppressed by $\left|U_{N \epsilon}\right|^{4}$ and $\left|U_{N \epsilon}\right|^{2}$ respectively compared to the unsuppressed $Z$ decay into $S U(2)_{L}$ doublet states. Limits from lepton universality [62] demand that $\left|U_{N \ell}\right|^{2}<0.1$ for all generations and masses, and compared to $Z$ decays, the production rates above are small. The decay $Z \rightarrow N \bar{\nu}$ is also passible [63], but is also highly suppressed by a small coupling factor. Therefore, the following searches urill not consider massive neutrinos $N$ in $S U(2)_{L}$ singlets, the production rate is too small.

\subsubsection{Neutrino Angular Distribution}

The angular distribution for sequential Dirac neutrino pair-production can be calculated using Eq. 8:

$$
\left.\frac{d \sigma\left(\nu^{D} \bar{\nu}^{D}\right)}{d \cos \theta}=\frac{G_{F}^{2}|R(s)|^{2}}{64 \pi s} \cdot \beta\left[\left(1-4 \bar{s}_{W}^{2}+8 \bar{s}_{W}^{4}\right)\left(1+\beta^{2} \cos ^{2} \theta\right)\right]+2\left(1-4 \bar{s}_{W}^{2}\right) \beta \cos \theta\right],
$$

with $R(s)$ the form of the $Z$ resonance from Eq. 9 and $\bar{s}_{W}^{2}$, the effective value of $\sin ^{2} \theta_{W}$ after radiative corrections defined earlier. For comparison, the sequential Majorana distribution is:

$$
\frac{d \sigma\left(\nu^{M} \nu^{M}\right)}{d \cos \theta}=\frac{G_{F}^{2}|R(s)|^{2}}{64 \pi s} \cdot \beta^{3}\left[\left(1-4 \bar{s}_{W W}^{2}+8 \bar{s}_{W}^{4}\right)\left(1+\cos ^{2} \theta\right)\right]
$$

The Dirac angular distribution is approximately proportional to $\left(1+\cos ^{2} \theta\right)$ as $\beta \rightarrow 1$, and becomes isotropic as $\beta \rightarrow 0$. In contrast, the Majorana angular distribution behavior in angle is independent of mass and always proportional to $\left(1+\cos ^{2} \theta\right)$. The differences between the two distributions is small for low masses, while the differences can lead to noticeable disparities in geometric acceptances for high masses.

\subsubsection{Neutrino Decay}

If the pattern of leptons masses in isodoublets in the first three generations is not followed, and $m_{L}<m_{\nu_{4}}$, then $\nu_{4}$ will decay through a virtual $W^{+}$to $L^{-}$and a fermionantifermion pair. The $L^{-}$will be stable unless there is significant mixing of the $L^{-}$to $e, \mu$, or $\tau$. A limit excluding stable fourth generation charged leptons has been set at $m_{L}>36.3$ $\mathrm{GcV} / \mathrm{c}^{2}$ at $95 \% \mathrm{CL}$ [64] making this decay channel unlikely. The case of $L^{-}$mixing will not be considered.

If, as in the first three generations, $m_{v_{4}}<m_{1,}$, then $\nu_{4}$ is either stable or will decay through the emission of a $W^{*}$ into a charged lepton $\ell$ with the standard coupling strength 
multiplied by $U_{l 4}$ if there is any mixing to the lighter generations as shown in Fig. $17(a)$. With mixing, the weak charged current decay modes $\nu_{4} \rightarrow W^{n} \ell ; \ell=e, \mu, \tau$ are then possible. For sequential neutrinos, FCNC decays through $Z$ emission as shown in Fig. 17(b) are highly suppressed by the GIM mechanism (neutrinos $N$ which behave as singlets under weak isospin can, however, undergo FCNC decays) as described earlier, and are not considered. If $m_{t}>M_{Z} / 2$, then the $W^{*}$ can decay into each of the three lepton doublets or two quark
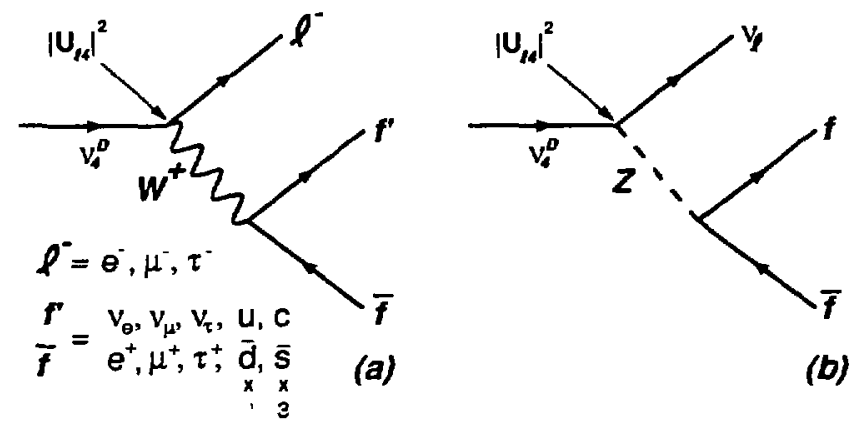

Figure 17: Feynman diagrams describing decay of massive sequential Dirac neutrinos (a) via the allowed charged current; and (b) highly suppressed FCNC decay.

doublets (with three colors each) and the decay rate is given by:

$$
\Gamma\left(\nu_{4}^{D} \rightarrow \ell^{-} X\right)=9\left|U_{\ell 4}\right|^{2} \frac{G_{F}^{2} m_{\nu}^{5}}{192 \pi^{3}} \mathcal{F}
$$

where $\mathcal{F}$ is a phase suppression factor [65] for massive final state particles which differs from unity only when one or more of the final state particles is a $\tau$ lepton or aquark, and $m_{\nu_{4}}$ is relatively small. The lifetime of $\nu_{4}^{D}$ can be expressed in terms of the $\mu$ lifetime as:

$$
\tau\left(\nu_{4} \rightarrow \ell^{-} X^{+}\right)=\left[\frac{m_{\mu}}{m_{\nu_{4}}}\right]^{5} \frac{\tau(\mu \rightarrow e \nu \bar{\nu}) B r\left(\nu_{4} \rightarrow \ell^{-} e^{+} \nu\right)}{\left|U_{\ell 4}\right|^{2} \mathcal{F}}
$$

Majorana neutrinos can decay via both the modes shown in Fig. 18 because of CPT conservation. No interference occurs between the diagrams because the final states are distinct. The predicted total lifetime will be simply one-half the corresponding Dirac neutrino decay since for every Dirac neutrino decay, there is a criesponding Dirac antineutrino 

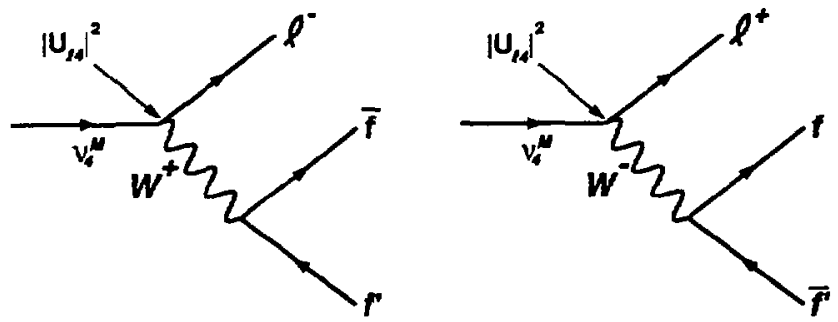

Figure 18: Possible decay modes for a Majorana neutrino.

decay of equal width, both of which are allowed for the Majorana neutrino. That is, for equal mixing matrix elements, $\tau\left(\nu_{4}^{D}\right)=2 \tau\left(\nu_{4}^{M}\right)$.

The $\nu_{4}$ mean decay length in the CM frame is

$$
\ell_{\nu_{4}}=\beta \gamma c \tau_{\nu_{1}}
$$

where $\gamma=1 /\left(1-\beta^{2}\right)^{1 / 2}$ and $c$ is the speed of light. Figure 19 shows the mean decay lengths of $\nu_{4}$ particles as a function of mass and mixing matrix element.

Possible $\nu_{4} \bar{\nu}_{4}$ event topologies can be categorized into three general classes depending on the mass and the lifetime:

\section{Short-lived Neutrinos}

Large mixings to the lighter generations leads to prompt $\nu_{4}$ decays (i.e. mean decay lengths less than $1 \mathrm{~cm}$ ). At least one charged lepton is always present in the decay products of each $\nu_{4}$, and as $m_{\nu_{4}}$ increases, this lepton will become more and more isolated from the other decay products. Note from Fig. 18 that Majorana neutrino decay can lead to samesign isolated dilepton events in contrast to Dirac neutrinos whose decays can lead only to opposite-sign isolated dilepton events.

\section{Long-lived Neutrinos}

For small but non-zero values of mixing, the $\nu_{4}$ will bave a long mean decay length resulting in decays in the detector volume and the observation of detached vertices. 


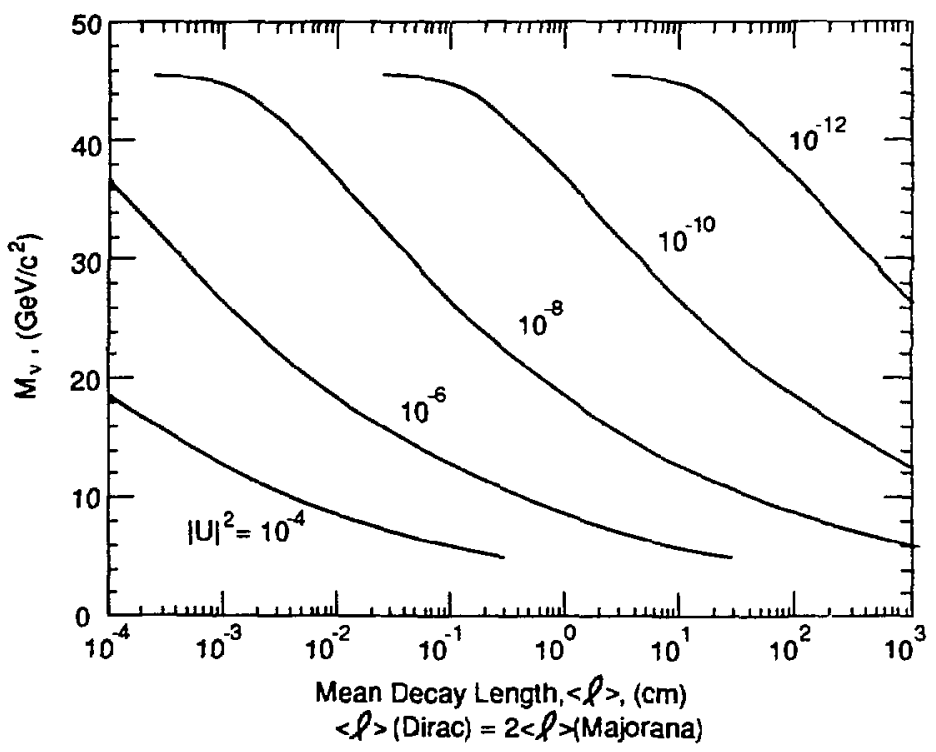

Figure 19: Mean decay lengths of massive Dirac and Majorana neutrinos as a function of mass and mixing matrix element.

\section{Very Long-lived or Stable Neutrinos}

For tiny or zero mixing values, the $\nu_{4}$ particles can escape the detector before decaying. Their presence can be inferred by this $Z$ decay mode increasing the width of the $Z$ resonance. These decays would contribute to $Z$ decays which are not visible in the detector, but which increase the "invisible" width of the $Z$. Splitting the total $Z$ width into a visible and invisible width,

$$
\Gamma_{\text {tot }}=\Gamma_{\text {vis }}+\Gamma_{\text {invis }}
$$

a stable $\nu_{4}$ (no mixing, therefore $\nu_{4} \equiv \nu_{L}$ ) will add a contribution of

$$
\Delta \Gamma_{\text {invis }}=166 \mathrm{MeV} \cdot \begin{cases}\beta\left(3+\beta^{2}\right) / 4 & \text { Dirac; } \\ \beta^{3} & \text { Majorana, }\end{cases}
$$

only to $\Gamma_{\text {invis. }}$ For finite decay lengths, there will be some efficiency for $\nu_{4}$ events to contribute to $\Gamma_{\text {vis }}$, while the contribution to $\Gamma_{\text {irvis }}$ will not be as large. Mass limits will be 
set using the resonance parameters of the $Z$ measured [66] with the Mark II detector. For unstable, promptly decaying $\nu_{4}$ events, the contribution will be mostly to $\Gamma_{\text {vis }}$. However, for these events, the direct searches described above are more efficient.

\subsubsection{Present $\nu_{4}$ Mass Limits}

A massive neutrino mixing with known neutrinos $\nu_{\ell}$ would reduce the $\nu_{\ell}$ 's effective weak interaction coupling strength by a factor $\left(1-\left|U_{\ell q}\right|^{2} / 2\right)$ and universality arguments [62] limit mixings at the $2 \sigma$ level to: $\left|U_{e 4}\right|^{2}<0.043,\left|U_{\mu 4}\right|^{2}<0.008$, and $\left|U_{\tau 4}\right|^{2}<0.30$ for new Dirac sequential neutrinos. An excellent review of exclusion regions for a low mass $\nu_{4}$ can be found in Ref. [67] and present exclusion regions for large mass $\nu_{4}$ are summarized in Fig. 20 for $100 \%$ mixing to $\nu_{e}$. The exclusion regions for $100 \%$ mixing to $\nu_{\mu}$ and $\nu_{\tau}$ are similar, except the CELLO result was not extended to mixing to $\nu_{\tau}$.

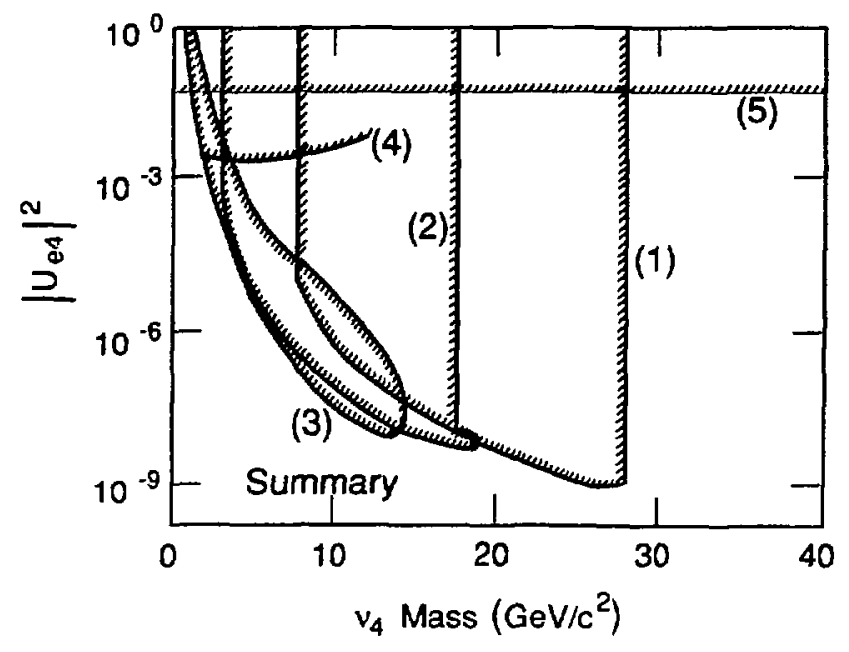

Figure 20: Examples of 95\% CL excluded regions for a Dirac sequential $\nu_{4}$ ( $100 \%$ mixing to $\nu_{e}$ ) in the $m_{\nu,}$ versus mixing matrix el=ment $\left|U_{e 4}\right|^{2}$ plane given by (1) AMY, Ref. [68], (2) CELLO, Ref. [69], (3) Mark II secondary vertex search at PEP, Ref. [70], (4) monojet searches at PEP, Ref. [71], and (5) e- $\mu$ universality, Ref. [62]. 


\subsection{Heavy Charged Lepton $\left(L^{-}\right)$}

Fig. 10 that shows the partial widths of new heavy fermions as a function of mass clearly illustrates the relatively small production rate of $L^{-}$compared to $t, b$, and $\nu_{4}$. The small data set of $528 Z$ decays collected by the Mark II precludes direct searches for $L^{+} L^{-}$events to set significant mass limits. However, $L^{-}$can potentially be detected by an increase of $\Gamma_{Z}$ if $m_{\nu_{L}}<m_{L}<M_{Z} / 2$ and $L^{-} \rightarrow \nu_{L} W^{*}$ is the dominant decay mode. It is also assumed that the neutrino is stable with no mixing $\left(\nu_{4}=\nu_{L}\right)$ to avoid the complication of mixing in both the charged lepton and neutrino sectors. This indirect method is also sensitive to events with particularly small mass differences $\left(\delta=m_{L}-m_{\nu_{L}}\right)$ where direct detection could be difficult [72]. If both $\nu_{L}$ and $L^{-}$are kinematically accessible through $Z$ decay, then $\Gamma_{Z}$ will increase by:

$$
\Delta \mathbf{r}_{Z}=\boldsymbol{\Gamma}_{L}+\boldsymbol{r}_{\nu_{L}}
$$

It is the substantial size of $\Gamma_{\nu_{L}}$ which makes this method sensitive to significantly large values of $m_{L}$ for the relatively small data set.

\subsubsection{Present $L^{-}$Mass Limits}

Previous aearches have set an experimental limit of $m_{L}>30 \mathrm{GeV} / c^{2}$ at $95 \%$ CL from $e^{+} e^{-}$collision [73], and a less direct limit of $m_{L}>41 \mathrm{GeV} / c^{2}$ at $90 \% \mathrm{CL}$ from pp collision [74] assuming that $v_{L}$ is massicso. These limits degrade as $\nu_{L}$ increases in mass, and [72] contains a comprehensive review of limits with $m_{\nu_{L}} \neq 0$. 


\section{Chapter 3}

\section{Experimental Apparatus}

The SLAC Linear Collider (SLC) provides colliding $e^{-}$and $e^{+}$beams at a center-ofmass energy $E_{\mathrm{cm}}$ of approximately $91 \mathrm{GeV}$, the mass of the $Z$. This analysis uses data collected by the Mark II detector that occupies the sole interaction region of the SLC. The Mark II detector was upgraded extensively after productive runs at the SPEAR and PEP storage rings, and the upgraded components first tested during trial runs at PEP. The detector was physically moved from a PEP interaction region to the SLC collision point in 1987, followed by a lengthy and difficult commissioning of the SLC. The first hadronic decay of the $Z$ ever observed occurred in the Mark II detector on April 11, 1989, and the data $\left(19.7 \mathrm{nb}^{-1}\right)$ for this thesis were collected in the time period from April 1989 to October 1989.

This chapter describes the SLC machine and the Mark II detector, stressing components used in this analysis.

\subsection{The SLAC Linear Collider (SLC)}

The SLC [75] is a novel electron-positron accelerator and a prototype of a new generation of colliding-beam accelerators. Linear colliders are a way to overcome the scaling law for electron colliding-beam circular storage rings, that, because of synchrotron radiation, have a size and cost which increases roughly as the square of $E_{c m}$. Linear colliders have no synchrotron radiation emitted in the acceleration process; therefore, the size of these devices increases as the first power of $E_{\mathrm{cm}}$. The SLC has been a success in both proving the viability of the linear collider concept and demonstrating its ability as a tool for high 
energy particle physics research, in particular, for studying the $z$ resonance and decays of the $Z$.

\subsubsection{Description}

Twenty years ago, the two mile long SLAC linear accelerator (LINAC) was first used to accelerate electrons for collision with protons (hydrogen) in fixed target experiments. Later, the LINAC was used to separately accelerate electrons and positrons for the "filling" of storage rings where the electrons and positrons would collide head-on as they coasted in opposite directions in the $\mathrm{r}$ 1g. Ruther than a linear collider in the truest sense (two linear accelerators pointing at eacin other), the SLC shown in Fig. 21 is a clever adaptation of the pre-existing SLAC LINAC. The LINAC was upgraded with new high-powered klystrons, the addition of damping rings, and the construction of beam transport lines (ARCs) to bring the intense, very small beams into head-on collision.

In a iypical operation cycle, $e^{-}$and $e^{+}$bunches with about $10^{10}$ particles in each bunch sre first circulated in small storage rings where they are "cooled" as their transverse emittance is damped by syachrotron radiation. After pulse compression which reduces the bunch lengths to $1.5 \mathrm{~mm}, 3 e^{+}$and a $e^{-}$bunch are simultaneously accelerated down the INAC to reach energies of about $47 \mathrm{GeV}$. Two-thirds of the way down the LINAC, a third trailing $e^{-}$bunch at an energy of $33 \mathrm{GeV}$ is made to collide with a station ry tungsten target to produce positrons which are returned to the front end of the LINAC to join electrons produced at a thermionic sun for the next machine cycle. The original bunches are then switched to separate ARCs where they lose about $1 \mathrm{GeV}$ of energy through the emission of synchrotron radiation in the process of being steered towards head-on collision. The bunches pass through a complicated transport section called the Final Focus system that corrects chromatic aberrat:ons and demagnifies the transverse size of the bunches from about $1 \mathrm{~mm}$ to a few microns before colliding at the interaction point (IP) surrounded by the Mark II detector. After the $e^{-}$and $e^{+}$bunches pass through each other, they are "licked" into extraction lines and deposited into beam dumps. This "single-pass" operation is in sharp contrast to storage rings where bunches intersect billions of times as they coast around the ring in opposits directions.

The luminosity $\mathcal{L}$ of th SLC is given by:

$$
\mathcal{L}=f \cdot \frac{N^{+} N^{-}}{4 \pi \sigma_{s} \sigma_{y}} \cdot H(D)
$$




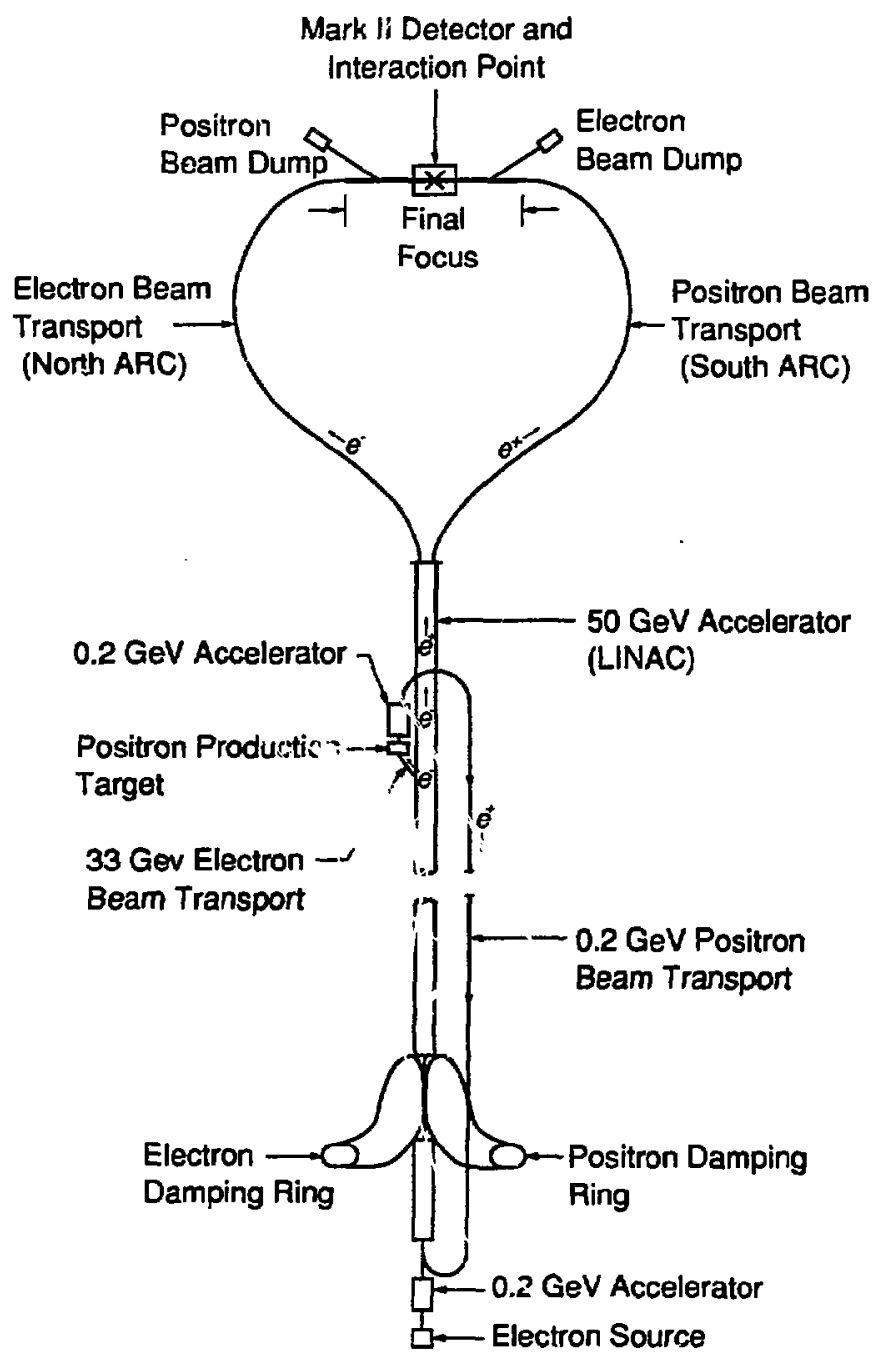

Figure 21: Schematic layout and operation of the SLC. 
where $f$ is the frequency of collisions, $N^{-(+)}$is the number of particles in the $e^{-}\left(e^{+}\right)$bunch, $\sigma_{x}$ and $\sigma_{y}$ are the transverse beam dimensions, and $H(D) \geq 1$ is a "disruption factor". This disruption factor provides an enhancement of luminosity through the electromagnetic focussing of one beam as it passes through the other. Due to power considerations and kicker capabilities, the frequency of collisions was limited to $60 \mathrm{~Hz}$ at the SLC, necessitating micron size beams at the interaction point to achieve reasonable luminosities. Operational parameters achieved [76] for the data set considered are listed in Table 6. It should be

Table 6: SLC Machine Parameters

\begin{tabular}{lcc}
\hline \hline Parameter & Achieved & Design \\
\hline Repetitior rate, $f$ & $60 \mathrm{~Hz}$ & $120 \mathrm{~Hz}$ \\
Intensity, $N^{ \pm}$ & $1.8 \times 10^{10}\left(N^{-}\right)$ & $6 \times 10^{10}$ \\
& $1.6 \times 10^{10}\left(N^{+}\right)$ & \\
Spot Sizes, $\sigma_{x}, \sigma_{y}$ & $3.3 \mu \mathrm{m}$ & $1.65 \mu \mathrm{m}$ \\
Disruption factor, $H(D)$ & 1 & 3 \\
Luminosity, $\mathcal{C}\left(\mathrm{cm}^{-2} \mathrm{sec}^{-1}\right)$ & $1.3 \times 10^{28}$ & $4 \times 10^{30}$ \\
$Z$ Rate & $1.5 / \mathrm{hr}$ & $470 / \mathrm{hr}$ \\
\hline
\end{tabular}

stressed that the values in Table 6 were reproducibly achieved but not simultaneously with good efficiency, so that a luminosity of $1.5 \mathrm{Z} / \mathrm{hr}\left(1.3 \times 10^{28} \mathrm{~cm}^{-2} \mathrm{sec}^{-1}\right)$ was generally considered 'good' running.

\subsubsection{Machine Backgrounds}

The problem of machine backgrounds had the potential to prevent the Mark II detector from acquiring usable data. These backgrounds arise when stray beam particles strike apertures in the Final Focus (FF) system creating electromagnetic debris, penetrating muons, and slow neutrons. These extraneous particles can be numerous enough to confuse and saturate the pattern recognition capabilities of the Mark II. Reduction of these backgrounds requires extensive shielding and protection of the Mark II detector along with efficient collimation of the beams.

Initially, primary collimation of the beams was provided in the FF, but it was impossible to operate the detector due to the large flux of electromagnetic debris and muons. These were caused when particles in the tails of the spatial distributions of the bunches struck 
collimators. A loss rate of 1 per $10^{4}$ particles in the FF was sufficient to prevent data-taking with the Mark II detector. The penetrating muon flux, which is resistant to shielding, is now controlled by large blocks of magnetized iron (muon toroids) mounted in the FF which sweep locally produced muons away from the detector. The primary collimation is now further upstream at the end of the LINAC and in the ARCs [77]. Even with these measures, it was often high machine backgrounds which imposed an upper limit on the useful luminasity of the SLC.

\subsubsection{Extraction Line Energy Spectrometers}

To determine $E_{\mathrm{cm}}$ to accurately measure the $Z$ resonance and calculate expected cross sections, the energy of each beam $E_{\text {beam }}$ needs to be known precisely. In a storage ring, the energy of the beams is known from the radius of the stored orbit and the field strengths of the bending magnets. This cannot be done at single-pass collider. Instead, after the $e^{-}$and $e^{+}$bunches pass through each other at the IP, energy spectrometers [78] in each extraction line measure the energy of the particles in the $e^{-}$and $e^{+}$bunches before they strike their respective beam dumps. A pulse-to-pulse measurement of $E_{\mathrm{cm}}$ with an absolute error of $\pm 35 \mathrm{MeV}$ and relative error of $\pm 27 \mathrm{MeV}$ is achieved.

In the energy spectrometer, the beam passes through three dipole magnets as shown in Fig. 22. The first small magnet sweeps the beam horizontally, and creates a horizontal

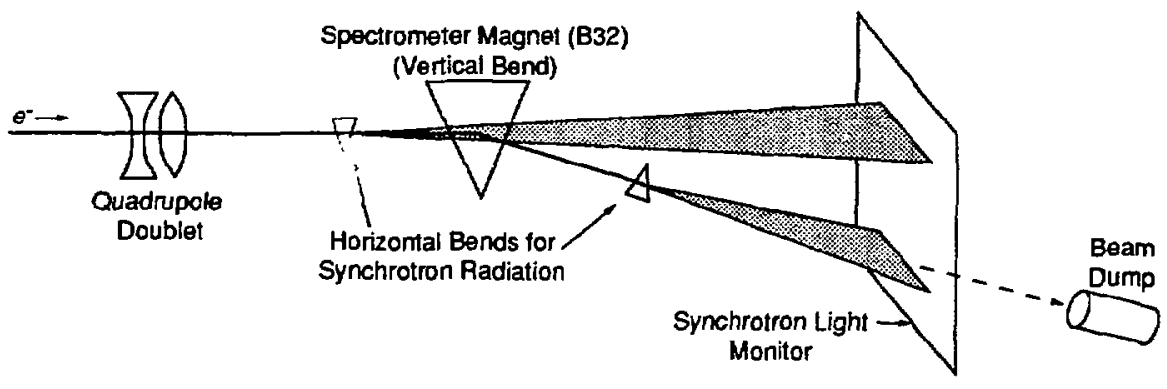

Figure 22: Schematic layout of the extraction line energy spectrometer.

swath of intense synchrotron radiation which is imaged as a stripe on a phosphorescent screen $15 \mathrm{~m}$ downstream. The large magnet B32 with a precisely measured field then 
bends the beam vertically by an angle $\theta=(0.29978) \int B d \ell / p$ where $\int B d \ell$ is the field integral of B32 in kG.m and $p$ is the beam momentum $(\mathrm{GeV} / c)$. The next small dipole then sweeps the bent beam horizontally creating a swath of synchrotron radiation parallel to the first, and imaged as another stripe on another phosphorescent screen. Measuring the distance between the two stripes (about $26 \mathrm{~cm}$ ) on the screens, and knowing the distance to the magnetic center of B32, the energy of the beam can be extracted. The width of the synchrotron stripes on the screens provides a $10 \%$ measurement of the energy spread of the particles in each bunch, typically 100 to $200 \mathrm{MeV}$.

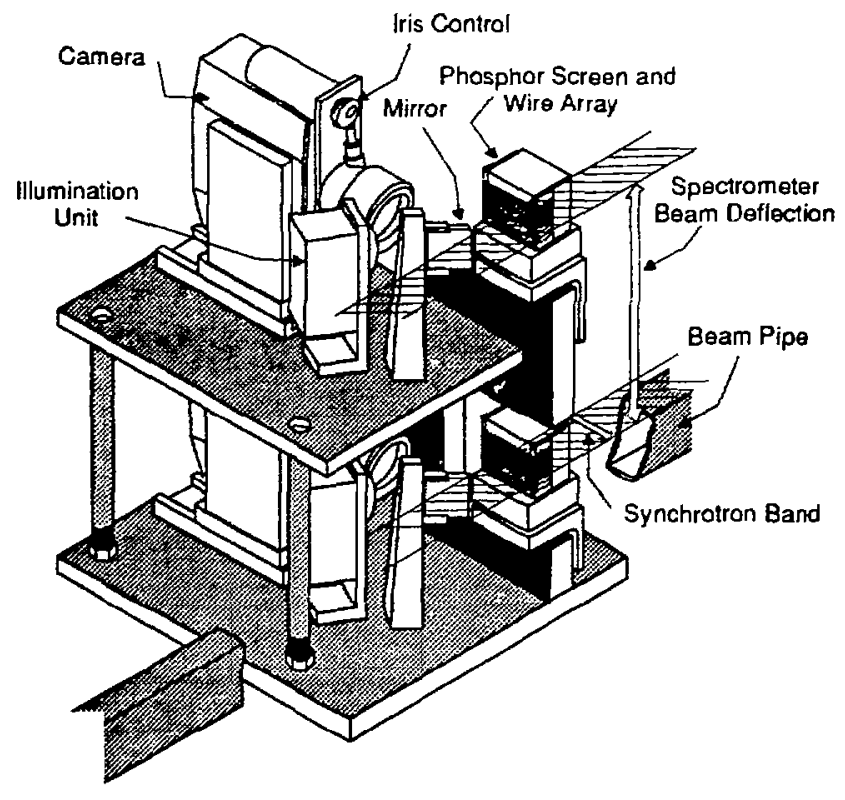

Figure 23: The phosphorescent screen monitor (PSM).

The absolute field integral $\int B d \ell$ of the two large magnets (B32) were accurately measured [79] before they were installed. $\int B d \ell$ was determined to an accuracy of 72 part per million (ppm) with a point to point variance of $54 \mathrm{ppm}$. A flipcoil mounted permanently inside B32 was simultaneously calibrated and provides field monitoring during running with an accuracy of $4.2 \mathrm{ppm}$. In situ NMR probes and current monitors provide redundant cross 
checks.

The separation between synchrotron stripes and their widths is measured using a Phosphorescent Screen Monitor (PSM) shown in Fig. 23. Two identical cameras view phosphorescent screen targets mounted a known distance apart. A fiducial array of $100 \mu \mathrm{m}$ wires with a spacing of $500 \mu \mathrm{m}$ between centers and backed by a phosphor screen constitutes each target. Light is emitted when the synchrotron radiation of maximum energy of $3 \mathrm{MeV}$ strikes the phosphor, and the video image of the light stripe parallel to the fiducial wires is digitized. The readout of the video digitizers is part of the CAMAC system readout for the entire Mark II detector and, after background subtraction, the Mark II online VAX computer fits gaussians to the stripe digitizations. The total systematic error on the distance (about $26 \mathrm{~cm}$ ) between the measured centroids of the stripes is estimated to be less than $28 \mu \mathrm{m}$.

The end result is that $E_{\mathrm{cm}} \pm 35 \mathrm{MeV}$ is recorded for each event logged by the Mark II detector.

\subsection{The Mark II Detector at the SLC}

The Mark II shown in Fig. 24 is a general purpose particle detector providing excellent tracking, electromagnetic calorimetry down to $15 \mathrm{mrad}$, and good lepton/hadron separation; however, it lacks hadronic calorimetry and $\pi / K / p$ separation above $2 \mathrm{GeV} / c$. An overview of the detector is first given, and succeeding sections give a more detailed description of each major part of the detector that is important to this analysis. The detector trigger and data acquisition system is then described. A more detailed description of the entire Mark II can be found in Ref. [80] and references therein.

\subsubsection{Overview}

Moving outwards in radius from the interaction point, the following major detector components are encountered:

\section{Beampipe}

The beampipe is an aluminum cylinder with an inner radius of $3.37 \mathrm{crn}$ and a thickness of $0.76 \mathrm{~mm}$ resulting in 0.0085 of a radiation length at normal incidence. "Wire Flippers" are located inside the beampipe at $\pm 0.18 \mathrm{~m}$ in $z$ and use thin carbon fibers to measure single beam profiles and determine the beam position to first order. 


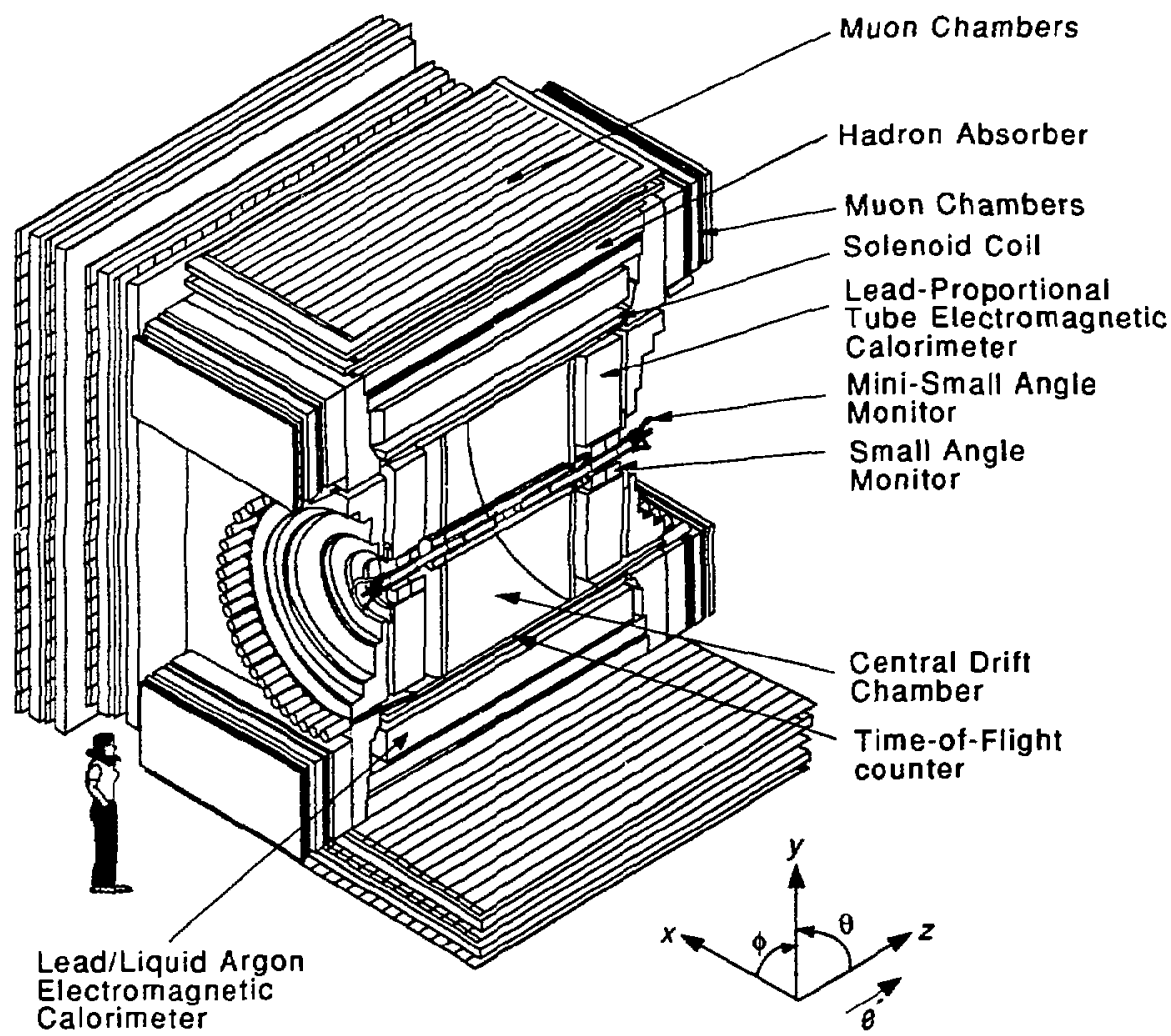

Figure 24: The Mark II Detector and detector coordinate system at the SLC.

They consist [81] of two wire-carrying heads at right angles to each other and can be remotely "llipped" into the beam path. The flippers add only about $7.5 \%$ to the scattering thickness of the beampipe above $20^{\circ}$ from the beam axis. There can, however, be as much as 1.7 radiation lengths of stainless steel, including structural material and langes, in front of parts of the endcap calorimeters.

\section{Drift Chamber}

A 72 layer jet-cell drift chamber provides precise information about the direction, 
curvature, and amount of ionization of charged particles produced at the IP. In conjunction with a solenoidal magnetic feld, a momentum measurement and limited particle identification of charged tracks is furnished by the drift chamber.

\section{Time-of-Flight}

At a radius of $1.52 \mathrm{~m}, 48$ slabs of plastic scintillator $3.0 \mathrm{~m}$ long and $4.5 \mathrm{~cm}$ thick parallel to the beam direction are viewed by photomultiplier tubes, one on each end of each slab. The transit time of particles from the IP to the scintillator is measured with a resolution of 220 pser, and provides charged particle identification and identification of cosmic rays.

\section{Solenoid Coil}

A conventional aluminum cylindrical coil produces a magnetic field of $4.75 \mathrm{kG}$ parallel to the $z$-axis of the detector. The coil is water-cooled and 1.3 radiation lengths thick. The produced magnetic field is uniform within the tracking volume to within $3 \%$ and has been mapped with an error of less then $0.1 \%$. Hall probes at the each end of the drift chamber monitor the field to an accuracy of $0.1 \%$.

\section{Barrel Calorimeter}

A lead/liquid argon sampling device with strip readout geometry provides electromagnetic calorimetry.

\section{Muon System}

A four layer, planar hadron absorber consisting of steel interleaved with proportional chambers covers $45 \%$ of the detector solid angle and allows high energy muons ( $p>1.8$ $\mathrm{GeV} / c)$ to be distinguished from charged hadrons.

Moving outwards in $z$ from the IP, the additional major systems encountered are:

\section{Endcap Calorimeter}

A lead/proportional tube sampling device supplies electromagnetic calorimetry in the angular region between approximately $15^{\circ}$ and $45^{\circ}$ from the beam axis.

\section{Small Angle Monitor}

A tracking section of drift tubes followed by a sampling calorimeter of lead and proportional tubes allows the measurement and identification of small angle Bhabha electrons and positrons for a luminosity measurement at a counting rate of 1.3 times the $Z$ decay rate. 


\section{Mini-Small Angle Monitor}

A tungsten/scintillator calorimeter also identifies Bhabha electrons and positrons for a luminosity measurement but at smaller angles where the counting rate is approximately seven times the $Z$ decay rate.

\subsubsection{Drift Chamber}

\section{Description}

The drift chamber (DC) consists of 12 concentric cylindrical layers of jet cells, each cell containing six sense wires. The innermost layer contains 26 such cells, and each subsequent layer contains 10 more cells than the previous layer. The layers alternate between axial layers parallel to the beam axis and layers arranged at an angle of $\pm 3.8^{\circ}$ to the beam axis to provide stereo information for tracking. The chamber extends in radius from $19.2 \mathrm{~cm}$ to $151.9 \mathrm{~cm}$ with an active length of $2.30 \mathrm{~m}$. The wires are strung between $5.1 \mathrm{~mm}$ thick aluminum endplates which are beld apart by a $2 \mathrm{rm}$ thick beryllium inner cylinder and $c$ $1.27 \mathrm{~mm}$ thick outer aluminum shell.

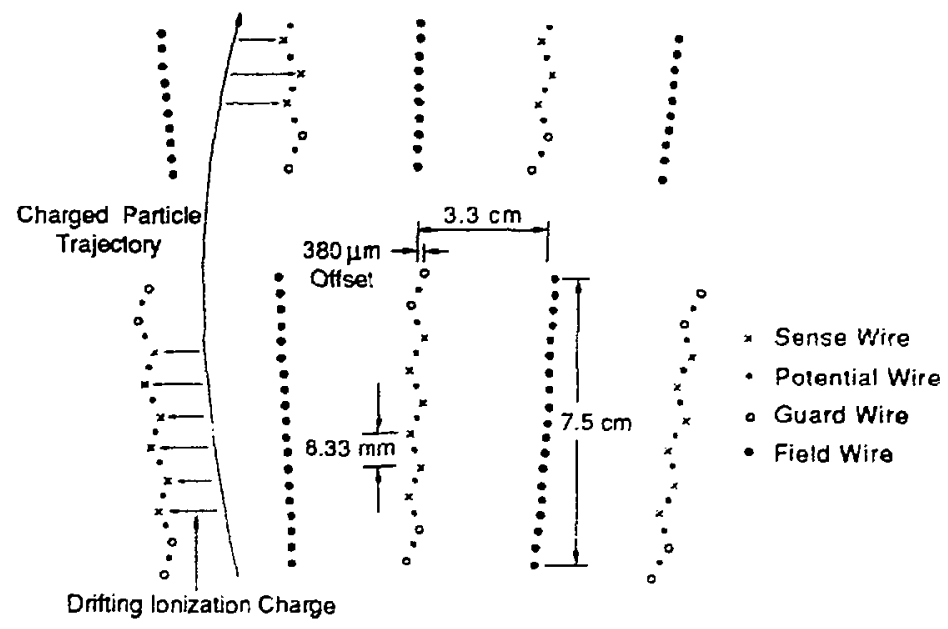

Figure 25: Drift chamber wire configuration.

The layout of wires in a cell is shown in Fig. 25. The average uncertainty in wire placement is $35 \mu \mathrm{m}$. The $30 \mu \mathrm{m}$ diameter gold plated tungsten sense wires are grounded 
and a typical voltage of $-4.5 \mathrm{kV}$ and $-1.5 \mathrm{kV}$ is applied through a resistor-divider chain i. the field and potential wires respectively. These voltages create a uniform drift held of $900 \mathrm{~V} / \mathrm{cm}$ in the cell and a gas gain of $2 \times 10^{4}$. A charged particle passing through the cell ionizes the gas $\left(89 \% \mathrm{Ar}, 10 \% \mathrm{CO}_{2}\right.$, and $1 \% \mathrm{CH}_{4}$, at a pressure of slightly more than 1 atmosphere) and the ionization charge drifts towards the sense wire with a velocity of $52 \mu \mathrm{ro} / \mathrm{usec}$. A measurement of the time taken for the ionization to reach the sense wires allows a precise measurement of the distance of the track from the wire. The sense wires are staggered by $\pm 380 \mu \mathrm{m}$ to distinguish tracks that pass to the right or left of the sense wire plane.

The sense wire signal is amplifed and discriminated and sent to two different digitizing systems to provide timing and pulse shspe information. Drift times are digitized by $96-$ channel LeCroy 1879 FASTBUS TDCs with multi-hit capability and a timing bin width of 2 ns. Pulse shapes are digitized by SLAC-designed [82] 16-channel $100 \mathrm{MHz}$ Flash ADCs (FADCs) with 6-bit resolution. The pulse shapes are integrated to determine the amount of ionization collected and provide a sample of energy loss $(d E / d x)$ of the charged track for particle identification. Pulse shape digitization also provides timing information which allows better double hit and two track separation when hits cannot be resolved by the TDCs.

SLAC Scanner Processors (SSPs) [83] in each FASTBUS crate perform zero suppression, pedestal corrections, and data formatting. The FADC SSPs also calculate the area integral and tiring of the pulse shape. The hast computer, a VAX 8600 , then reads out the preprocessed data stored in the SSPB via cable segments.

Calibration of the FASTBUS modules measures time propagation differences for each TDC channel, and pedestal and gain corrections for each FADC channel.

\section{Performance}

The position resolution of the DC ranges from $220 \mu \mathrm{m}$ for the longest drift distances down to a minimum of $130 \mu \mathrm{m}$ for tracks closer to the sense wire. Hits in a cell are used to find direction vectors (segments) for each charged track, one for each layer. Track-finding is accomplished [84] by finding clusters of segments in a $\rho-\phi$ plane where $\rho$ and $\phi$ are rcspectively the estimated track curvature and extrapolated azimuthal position for each segment. The track reconstruction efficiency is shown in Fig. 26 as a function of $\cos \theta$ and has been measured to be $>99 \%$ for isolated tracks traversing all 12 layers and measured 
Tracking Efficiency lor the Central Dritt Chamber

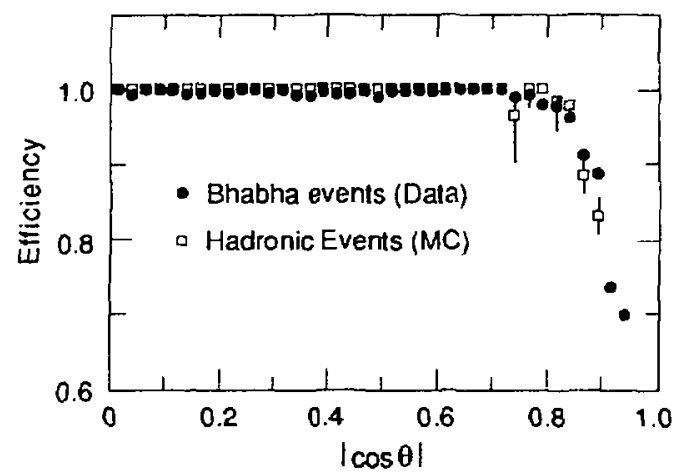

Figure 26: Track reconstruction efficiency for the drift chamber as a function of $\cos \theta$. The hadronic events (boxes) are from a Monte Carlo study at SLC energies; the Bhabha scattering events (dots) are from a sample of PEP data.

to be approximately $95 \%$ for tracks in hadronic $Z$ events.

In the FADC system, the SSPs apply a "difference of samples" algorithm [85] to the digitized sense wire pulse string to find leading and trailing edges for each hit and to separate overlapping pulses. The ability of the FADC system to resolve closely spaced tracks is shown in Fig. 27 compared to the separation using TDC hits alone.

A single track momentum resolution of

$$
\frac{\sigma(p)}{p}=\sqrt{(0.0046 p)^{2}+(0.019)^{2}}, \quad(p \text { in } \mathrm{GeV} / c)
$$

has been measured using Bhabha scattering events from PEP data in a $4.5 \mathrm{kG}$ magnetic field and verified using muon pairs from $Z$ decays at the SLC. The first term in Eq. 58 represents the intrinsic resolution of the DC, while the second term [86] arises from multiple scattering in material preceding the DC. This multiple scattering is not uniform in $\phi$, and increases when particles pass through the wire lippers devices mounted inside the beam pipe or through small diagnostic proportional-tube straw chambers mounted between the beam pipe and the DC. The momentum resolution improves if tracks are constrained to come from a single point (vertex constrained), but since this analysis considers tracks which may be decay products of a new, potentially long-lived particle, the vertex constrained momentum values are not used. 


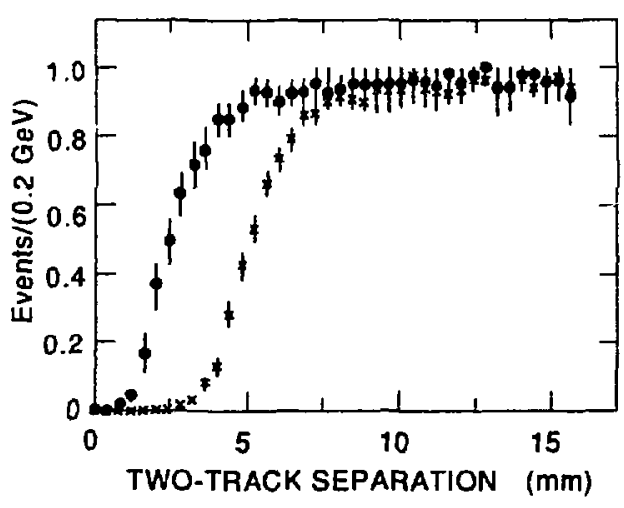

Figure 27: Efficiency for separating two tracks as a function of their distance spart for the drift chamber TDCs alone $\left(X^{\prime} s\right)$ and including FADC information (closed circles).

The FADCs provide limited discrimination of electrons, pions, bans, and protons for momentum between $1 \mathrm{GeV} / c$ and $8 \mathrm{GeV} / c$ through the measurement of average ionization loss $(d E / d x)$ of a charged track. 72 measurements of ionization are made for tracks traversing all 12 layers $(|\cos \theta|<0.6)$, each collested over a gas length of $8.33 \mathrm{~mm}$ for tracks perpendicular to the sense wires. The resolution achieved for minimum ionizing tracks at the SLC is $8.5 \%$ of the measured value. Particle identification is not used in this work, and more details of the $d E / d x$ system can be found in Ref. [87].

\subsubsection{Calorimetry}

Electromagnetic calorimetric coverage is furnished by the Liquid Argon Barrel Calorimeter (LA) and the Endcap Calorimeter (EC), each covering $64 \%$ and $22 \%$ of the detector solid angle respectively as shown in Fig. 28.

\section{LA Description}

The Liquid Argon Barrel Calorimeter [88] consists of eight identical and independent modules maintained at cryogenic temperatures ( $\sim 85 \mathrm{~K}$ ) by liguid nitrogen and an insulating vacuum of $10^{-6}$ in the aluminum casing of each module. The modules (each 


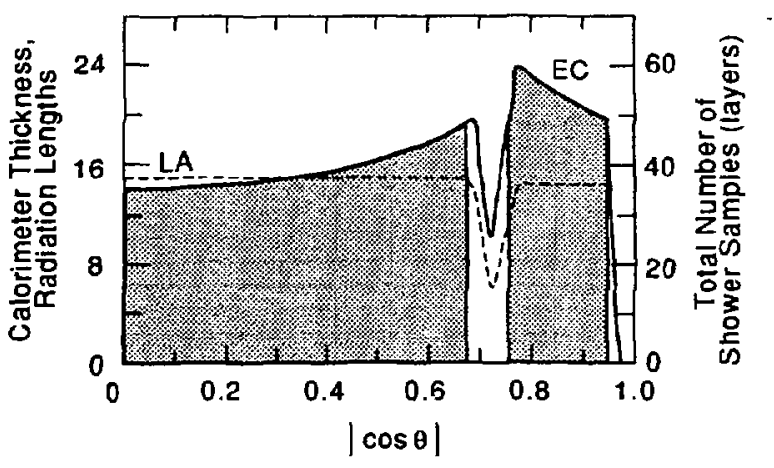

Figure 28: Total calorimeter thickness (solid line) and number if sarpling layers (dashed line) versus $\cos \theta$. The shaded area shows the re $e_{b}$ io: used for calculating the solid angle coverage.

$1.5 \times 3.8 \times 0.21 \mathrm{~m}^{3}$ in dimension) are arranged in an octagonal barrel surrounding the magnetic coil to cover the polar angle range of $|\cos \theta|<0.68$ and the full azimuthal angle $\phi$ except for $3^{\circ}$ gaps between pairs of modules.

Each module contains 37 planes of $2 \mathrm{~mm}$ thick lead separated by $3 \mathrm{~mm}$ gaps filled with liquid argon, resulting in a total of 14.4 radiation lengths (1.1 nuclear interaction lengths) of material at rormal incidence. Every second layer of lead is segroented into instrumented strips maintained at a voltage of $3.5 \mathrm{kV}$, and the remaining lead planes are grounded creating a $12 \mathrm{kV} / \mathrm{cm}$ electric field between lead planes. A photon or electron entering the calorimeter will shower electromagnetically in the lead planes ${ }^{\dagger}$ and create ionization electrons in the liquid argon gaps. The ionization charge drifts to the readout strips to produce a signal.

As shown in Fig. 29, the planes of readout strips are ganged together to reduce the number of electronic channels to 326 per module in the following manner:

F Planes: Nine planes have $3.8 \mathrm{~cm}$ wide strips aligned parallel to the $z$-axis to measure the azimuthal angle $\phi$ of the shower. They are ganged into three readout layers, F1 (front), F2 (middle), and F3 (back).

\footnotetext{
'Due to nuclear interaction processes, an entering hadron will ty pically deposil less cucrgy and intization due to nuclear interaction processes.
} 
T Planes: Six planes have $3.8 \mathrm{~cm}$ wide strips aligned perpendicular to the $z$-axis to mear sure the polar angle $\theta$ of the shower. They are ganged into two separate readout layers, T1 (front) and T2 (middle).

U Planes: Three planes have $5.4 \mathrm{~cm}$ wide strips arranged at $45^{\circ}$ to the $z$-axis to resolve ambiguities due to multiple showers. They are ganged into a single readout layer, $U$.

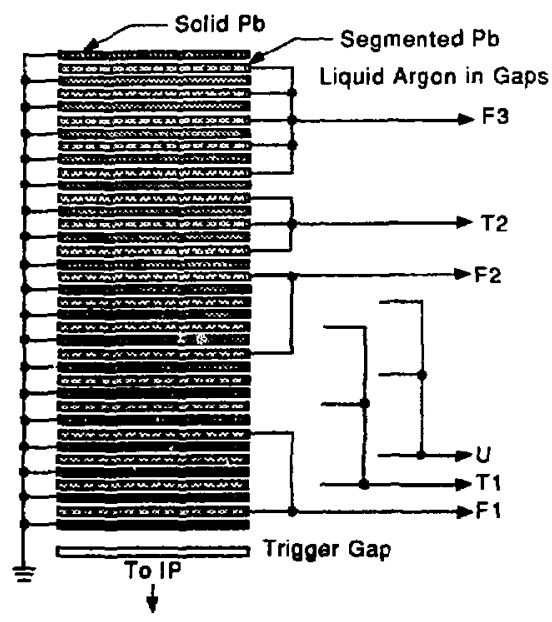

Figure 29: Ganging scheme for the layers in the liquid argon barrel calorimeter.

In addition, in the front of each module's lead stack, there are three $1.6 \mathrm{~mm}$ thick aluminum planes separated by $8 \mathrm{~mm}$ liquid argon gaps, known collectively as the "trigger gap". It is designed to minimize the probability of initiating a shower in order to identify showers starting in the magnet coil so that a correction for the energy lost in the coil can be applied to the observed energy in the calorimeter.

The signal on each readout strip is amplifed and shaped at the detector to drive an output line. The analog signals are sampled at the peak by CAMAC-based sample-andhold modules (SHAMs [89]) and digitized by 12-bit ADCs in BADC (Brilliant ADC [90]) microprocessors which also perform pedestal subtraction, gain corrections, and threshold cuts determined from periodic calibration of the electronics by the injection of a varying charge at the amplifier. The meraories of the BADCs are readout by CAMAC protocol by the hast VAX computer. 


\section{LA Performance}

Reconstruction of electromagnetic showers [91] is fairly elaborate. Basically, tracks found in the DC are projected into the calorimeter and any deposited energy in a narrow cone about that projection is associated with the charged track. Next, clusters of strips within each layer are found based on a threshold energy deposition and contiguity of strips. Neutral shower candidates are found from self-consistent combinations of clusters in different layers in the front half of the calorimeter. Any energy deposited in the back half of the calorimeter is added if it is geometrically consistent with an existing shower.

Figure 30 shows the energy measured by the LA system for $14.5 \mathrm{GeV}$ Bhabha scattered $e^{-}$and $e^{+}$at PEP with visible radiative Bhabha events excluded. From the width of the

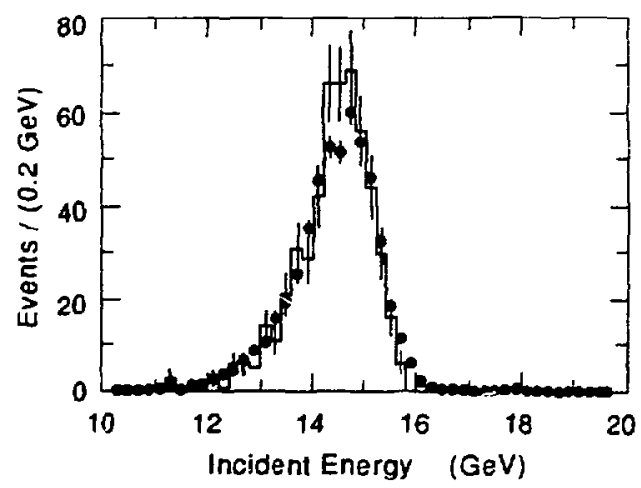

Figure 30: Measured energy distribution for the liquid argon barrel calorimeter for $14.5 \mathrm{GeV}$ Bhabha scatters $e^{-}$and $e^{+}$at PEP. The histogram is a Monte Carlo ${ }^{\circ}$ vilation of the detector response.

distribution at half maximum, $\sigma(E) / E=4.6 \%$. The non-gaussian low energy tail is due to inactive regions in the calorimeter. Saturation in the readout electronics for the trigger gap also degraded the resolution. The histogram of Fig. 30 shows a Monte Carlo simulation of the detector response taking account inactive regions and saturation effects. The saturation problem has since been corrected, and Fig. 31 illustrates the energy resolution of the LA at the SLC calculated using the above Monte Carlo simulation without saturation effects. The measured energy resolution for $\mathrm{e}^{+} \epsilon^{-}$pairs entering the LA at the SLC is consistent 
with these values. On average, the energy resolution of the LA system is:

$$
\frac{\sigma(E)}{E}=\frac{0.14}{\sqrt{E}}(E \text { in } \mathrm{GeV})
$$

with a shower position resolution of $\sigma_{\phi}=3$ mrad and $\sigma_{z}=0.8 \mathrm{~cm}$.

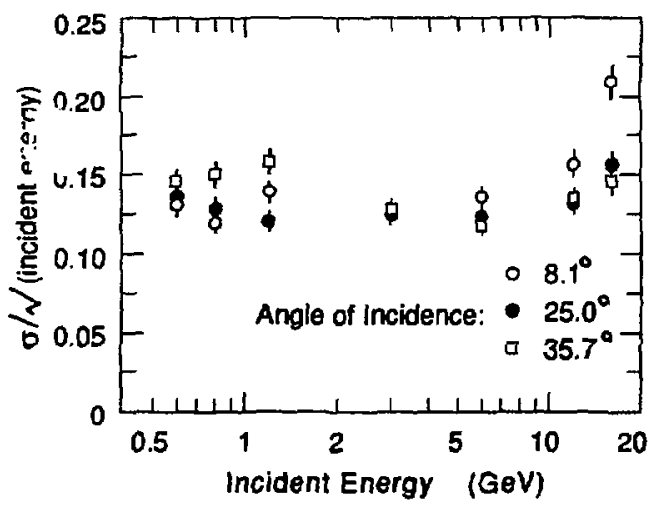

Figure 31: Monte Carlo simulation of the liquid argon barrel calorimeter energy resolution as a function of energy and angle of inciderce.

\section{EC Description}

The Endcap Calorimeter (EC [92]) consists of two annular modules (endcaps) with inner radii of $40 \mathrm{~cm}$ and outer radii of $146 \mathrm{~cm}$ situated at $\pm 1.37 \mathrm{~m}$ in $z$ to cover the angular region $0.707<|\cos \theta|<0.965$. Each module contains 36 layers of lead $0.28 \mathrm{~cm}$ thick, each separated by a plane of proportional tubes of rectangular $\left(0.9 \times 1.5 \mathrm{~cm}^{2}\right)$ cross section for a total of 18 radiat: four layers of tubes follows the pattern: vertical, horizontal, $+45^{\circ}$, and $-45^{\circ}$. This pattern is repeated for the first 20 layers, and the remaining 16 layers are alternately arranged horizontally and vertically.

A $50 \mu \mathrm{m}$ nickel-chromium alloy wire is strung through the center of each tube which is filled with the same gas mixture as the DC. Each wire is maintained at a voltage of $1.65 \mathrm{kV}$ and collects ionization charge from charged particles and electromagnetic showera 
passing through the gas. The gas gain of the system is monitored online with small tubes containing $\mathrm{Fe}^{55}$ sources at the inlet and outlet of the gas system.

The number of electronics channels are reduced to 1276 per endcap by ganging in a projective geometry giving 10 interleaved measurements of the longitudinal shower development. The proportional tube signals are amplified and shaped at the detector, then digitized and readout with the same SHAM-BADC combination in CAMAC as the LA system. The calibration procedure of the electronics is also similar to the LA system.

\section{EC Performance}

To develop shower reconstruction algorithms, one of the endcaps was tested in both pion and positron beams prior to installation with the response shown in Fig. 32. A study

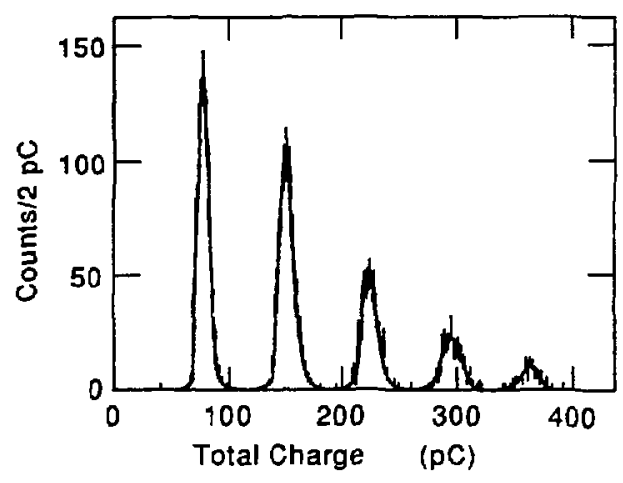

Figure 32: Response of the endcap calorimeter to test beam pulses containing between one and five $10 \mathrm{GeV}$ positrons. The five peaks are clearly distinguishable.

of Bhabha scattering events at PEP gives an energy resolution of

$$
\frac{\sigma(E)}{E}=\frac{0.22}{\sqrt{E}} \quad(E \text { in } \mathrm{GeV}\rangle
$$

with a position resolution of $0.27 \mathrm{~cm}$ in both the $x$ and $y$ directions. 


\section{Small Electromagnetic Shower Systems}

The requirement of hermeticity and low-angle coverage prompted the installation of four small detector systems which provide shower counter coverage in the solid angle not covered by the main calorimeters. The small counters at low-angles (Active Mask and Catcher, see Fig. 33) are generally swariped by extraneous signals from machine backgrounds and are used only as machine noise monitors.

For $|\cos \theta|<0.7$, at each junction between LA modules, a $3^{\circ}$ region in $\phi$ is not instrumented with calorimetry. Liquid Argon Hole Tagger counters are mounted on the outside surface of the liquid argon cryostat to fill in these "cracks" and to detect photons and electrong escaping through them. Each counter is a $2.5 \times 20 \times 165 \mathrm{~cm}^{3}$ slab of plastic scintillator faced with $1.9 \mathrm{~cm}$ of lead preradiator, which, along with other detector elements, gives a total of 6.0 radiation lengths of material preceding the scintillator. The scintillator is viewed at both ends by photomultiplier tubes. The crack between LA modules at $\phi=270^{\circ}$ could not be instrumented due to physical constraints. The Hole Taggers have proven useful in distinguishing $\mu^{+} \mu^{-}$pairs from $e^{+} e^{-}$pairs which ascape through the LA cracks.

\subsubsection{Luminosity Monitors}

The luminosity of the SLC is monitored by counting small-angle Bhabha scattering events detected by the Small-Angle Monitor (SAM) in the angular region $50 \mathrm{mad}<$ $\theta<160 \mathrm{mrad}$ and by the Mini-Small-Angle Monitor (Mini-SAM) in the angular region 15 mrad $<\theta<25$ mrad as shown in Fig. 33.

\section{SAM Description}

The SAM is a pair of modules with their front faces located at $\pm 1.38 \mathrm{~m}$ in $z$. As shown in Fig. 34, each module consists of a nine layer tracking section of drift tubes and a six layer (lead and proportional tubes) calorimeter section. A tungsten mask at $50 \mathrm{mrad}$ precisely defines the inner edge of the SAM angular acceptance.

The square drift tubes are $9.5 \mathrm{~mm}$ wide and contain the same gas as the DC at a pressure of slightly more than one atmosphere. Each tube has a $38 \mu \mathrm{m}$ gold-plated tungsten wire strung at the center of the tube and held at a voltage of $1.8 \mathrm{kV}$ with respect to the grounder outer wall. There are 30 tubes in each layer. The first layer is oriented horizontally, and the next two layers rotated $\pm 30^{\circ}$ with respect to the first. This pattern is repeated for all 


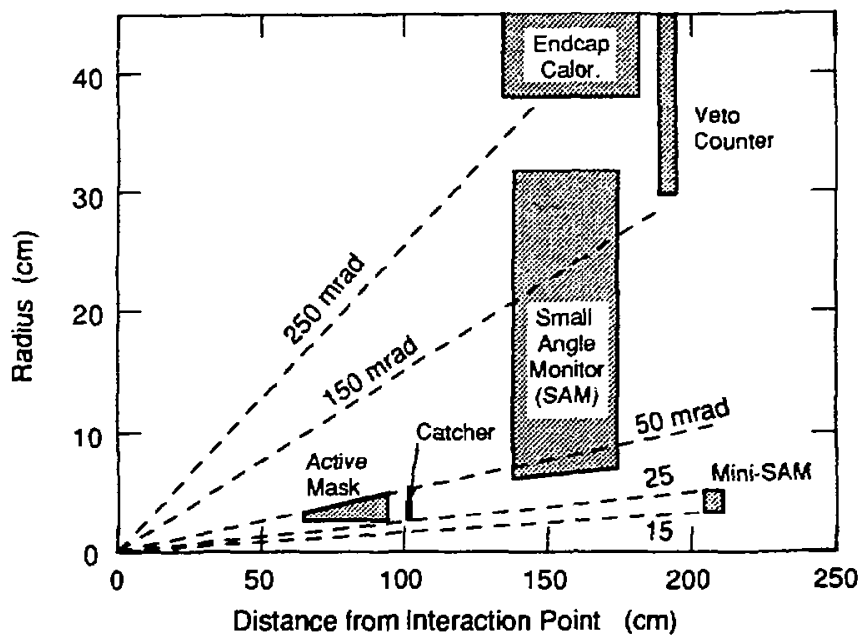

Figure 33: Layout of luminosity monitors and other small angle devices.

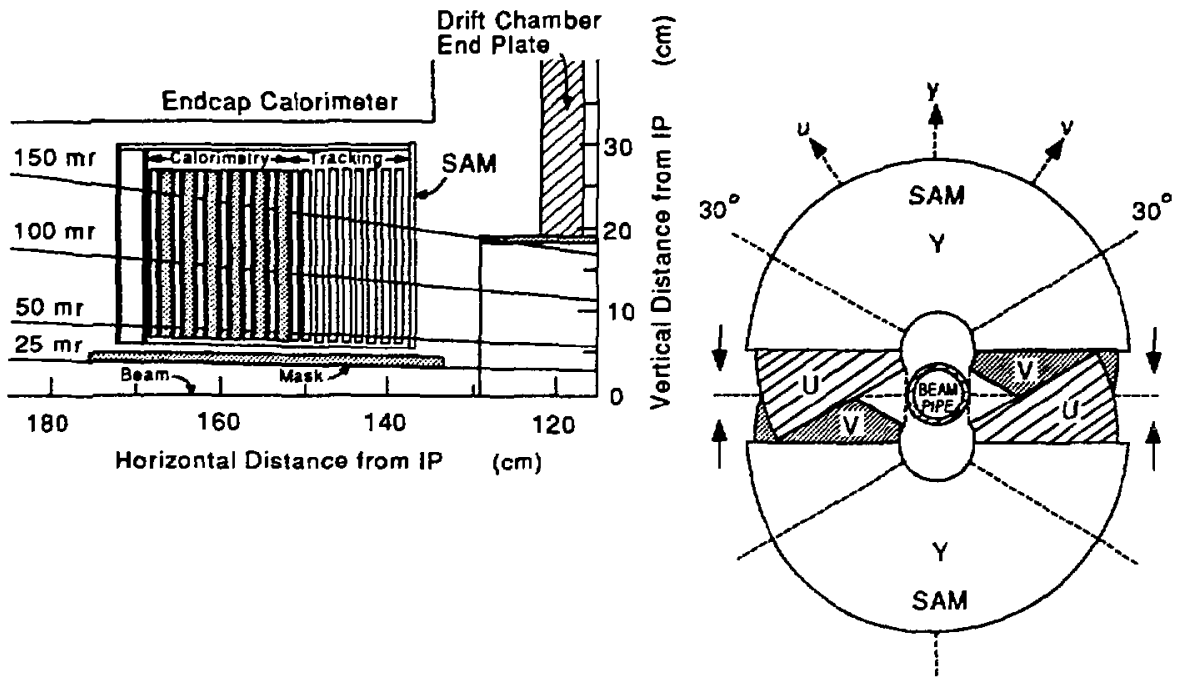

Figure 34: Side and front view of the Small-Angle Monitor and how it clamps around the beam pipe. 
the remaining layers of tubes. Each calorimetry section is six layers of $13.2 \mathrm{~mm}$ thick lead interleaved with layers of proportional tubes identical to the drift tubes, except that they are operated at a voltage of $1.7 \mathrm{kV}$. The lead and proportional tubes have a total thickness of 14.3 radiation lengths.

The timing signals from the drift tubes are converted by time-amplitude-converters (TACs [89]) to analog signals and these pulses in turn digitized and readout by BADC microprocessors described earlier. The signals from the proportional tubes are digitized and readout by a SHAM-BADC combination similar to that of the LA and EC systems. For calibration, pulses are injected into the system with variable time delays and constant pulse height for the TACs and with constant time delay and variable pulse heights for the SHAMs.

\section{SAM Performance}

From its response in a test beam of positrons with energies between 5 and $15 \mathrm{GeV}$ [93], the energy resolution of the SAM was found to be $\sigma(E) / E=0.45 / \sqrt{E}$ ( $E$ in $\mathrm{GeV}$ ) with a tracking angular resolution of $0.2 \mathrm{mrad}$.

At the SLC, machine backgrounds sometimes rendered the SAM tracking section useless, and the angles of the scattered Bhabhs tracks had to be measured using the calorimeter section with an angular resolution of 1 mrad. Fluctuations in longitudinal shower leakage from $45 \mathrm{GeV}$ Bhabha electrons and positrons degrade the energy resolution stated previously. The energy resolution for Bhabhas at the SLC is measured to be $\sigma(E) / E=0.15$. The sum of all the corrections for detector effects of the SAM leads to a $2 \%$ systematic error in the absolute cross section measurement (to be added in quadrature with the $2 \%$ uncertainty in the calculation of the radiative corrections to the Bhabha scattering cross section, details can be found in Ref. [94]). The cross section for Bhabha events in the SAM acceptance is roughly $20 \%$ higher than the visible $Z$ cross section.

\section{Mini-SAM Description}

The Mini-SAM consists of a north and south module, each divided into four azimuthal segments as shown in Fig. 35. The modules are formed of alternating layers of tungsten $0.79 \mathrm{~cm}$ thick and scintillator $0.64 \mathrm{~cm}$ thick, resulting in 15 radiation lengths of material. An additional 4.5 radiation lengths of preradiator (two layers of tungsten) precedes the first layer of scintillator. A photomultiplier tube views a wavelength shifter bar which runs the 


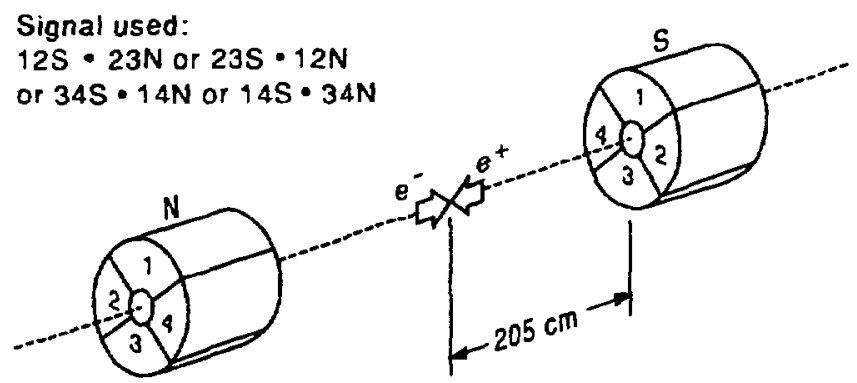

Figure 35: Geometry of the Mini-Small-Angle Monitor. As an example, in the 'Signal Used' definition, 12S means the signal sum of quadrants 1 and 2 being over a Bhabha threshold in the south monitor.

length of each azimuthal segment of each module and is optically coupled to the scintillator of that quadrant. Two conical masks of $5.08 \mathrm{~cm}$ thick tungsten sharply define angular acceptance windows.

The Mini-SAM resdout electronics consists of a SHAM IV'-BADC combination similar to that of the LA and EC systems.

\section{Mint-SAM Performance}

EGS [97] Monte Carlo simulations predict an energy resolution of $\sigma(E) / E \sim 0.35 / \sqrt{E}$ ( $E$ in $\mathrm{GeV}$ ) for the Mini-SAM, confirmed by test beam studies of a prototype module. Cosmic ray tests were used to set an energy scale for the actual Mini-SAM modules.

The Mini-SAM is particularly sensitive to machine background because of its location close to the beampipe. Timing information on the photomultiplier tube pulses aids in the distinguishing of Bhabha events from machine background debris striking the backs (sides away from the IP) of the Mini-SAM modules. Large backgrounds in the Mini-SAM decreases its efficiency for identifying Bhabhe events. This effect is discussed in more detail and parameterized in Ref. [98].

There were large uncertainties in the absolute cross section of Bhabha scatters into the Mini-SAM due to errors in the alignment of the acceptance masks. The Mini-SAM Bhabha rate is approximately eight times the visible $Z$ rate, and the Mini-SAM is used to

${ }^{1}$ The SHAM IV holds the integrated charge of an input pulse for subsequent digitization, while the: SHAM II used in the LA and EC systems holds the pulse height of an input pulse. 
measure relative luminosity over an energy scan, while the SAM is used to measure absolute luminosity.

\subsubsection{Trigger System}

At the SLC, the beam crossing rate ranges between $10 \mathrm{~Hz}$ and $60 \mathrm{~Hz}$ allowing sufficient time between every beam crossing* for fast electronics to decide for a particular beam crossing whether it is interesting enough to warrant triggering the Mark II data acquisition system to further process data for logging to magnetic tape. A CAMAC-based Master Interrupt Controller (MIC) module provides the interface between the trigger logic and the host VAX computer. The trigger logic results in a typical rate of $1-2 \mathrm{~Hz}$ for logging beam crossing events to tape. Deadtime imposed by the trigger processing time is negligible.

The Mark II trigger is the logical OR of a data, luminosity, cosmic ray, and random trigger.

\section{Data Trigger}

The data trigger uses information from charged tracks in the DC and energy information from the calorimeters to form its decision.

The charged track component of the data trigger uses a fast hardware track processor [95] to identify and count charged tracks in the 12 layers of the drift chamber. Track segments are first searched for in the cells of the DC by considering a cell "hit" if four of the six sense wires have signals detected by the TDCs (without considering the TDC timing information). The pattern of hits for each of the 12 layers are loaded into shift registers scanned by programmable "curvature" modules (see Fig. 36) which search for track candidates in the $x-y$ plane by requiring a certain number of hits (i.e. track segments) to fall into a curved "road" extending from the IP. Each module is designed to look for tracks of a certain range in radius of curvature, corresponding to a certain range in transverse momentum $p_{t}$.

For the data set considered, the modules were programmed to require that at least 8 out of the 10 first layers have hits contained in a road to count a track. In order to traverse at least eight layers, the track must be in the angular region of $|\cos \theta|<0.75$. In addition, it must have $p_{t}>150 \mathrm{MeV} / c$, the minimum transverse momentum searched for by the

\footnotetext{
-A beam erossing signal is provided to the trigger electronics by fast "beam pickoff" flectrodes $13.6 \mathrm{~m}$ ost rithes side of the $1 P$ which detect the particle bunches passing by.
} 


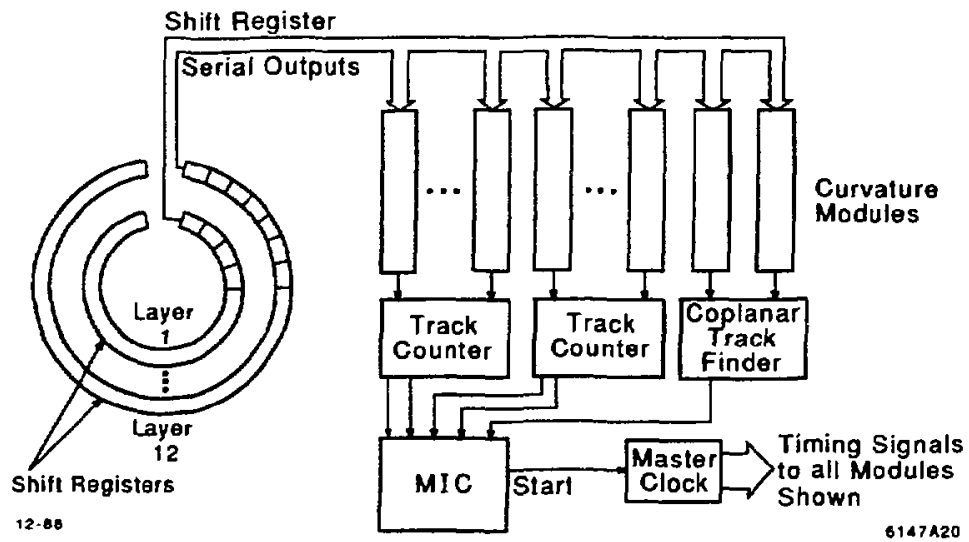

Figure 36: Block diagram of the charged track data trigger.

curvature modules. Finally, trackes must be separated by at least $10^{\circ}$ in azimuth to be counted as separate tracks. The charged particle trigger then requires at least two such trackes.

From Monte Carlo studies and a software emulation of the trigger hardware, the charged track trigger efficiency is $>99 \%$ for tracks passing through al 12 DC layers, and $97 \%$ for hadronic $Z$ events.

Another independent data trigger called the SSP Software Trigger (SST [96]) uses the pattern of energy deposition in the calorimetry to form its trigger decision. Analog sums of channels in the LA and EC calorimeters are digitized by LeCroy 1885N FASTBUS ADCs. A SLAC Scanner Processor (SSP) reads out the digital sums and uses algorithms in software to find clusters of energy deposition over adjustable software thresholds. This trigger is much more flexible than the older Total Energy Deposited (TED) trigger which now provides a redundant trigger and a cross check on SST trigger efficiency.

The current SST algorithm requires at least one cluster of energy greater than $2.2 \mathrm{GeV}$ in the LA or at least one cluster of energy greater than $3.3 \mathrm{GeV}$ in the EC system. The SST is emulated by offline software leading to an estimate of the energy-deposited trigger efficiency of $95 \%$ for hadronic $Z$ decays.

Together, the two triggers are estimated to be $99.8 \%$ efficient for hadronic $Z$ decays. 
Luminosity Trigger

The SAM and Mini-SAM can also trigger a readout of the detector for Bhabha scattering events for the monitoring of luminosity. Analog sums are made of the energy depesited in the front and back half of each of the two SAM modules, as well as the total energy deposited in each module. The SAM trigger requires at least $4 \mathrm{GeV}$ of energy in the front or back of a module, or $7 \mathrm{GeV}$ in an entire module, for both modules. An analog sum is made of the total energy deposited in each of the North and South Mini-SAM modules, and at least $20 \mathrm{GeV}$ of energy is required in both modules for a trigger. A programmable Memory Logic Module (MLM) forms the final luminosity trigger decision.

\section{Cosmic Ray Trigger}

Between beam crossings, a small time window is made available for cosmic ray triggers defined using the charged track trigger described previously, but only demanding one or more tracks. These cosmic ray events allow checking of the charged particle tracking performance. In addition, these events permit the determination of offline DC tracking and gas gain constants.

\section{Random Trigger}

A scaleable number of random beam crossing events are also allowed to trigger the detector readout to provide events for studying machine backgrounds and noise in the detector in an unbiased way.

\subsubsection{Data Acquisition System}

A typical hadronic $Z$ decay generates on the order of 50 kbytes of data from the Mark II detector and electronics. A VAX 8600 host computer reads CAMAC and FASTBUS data, and in addition, merges raw data with the results for online event tagging; logs event records to magnetic tape; logs tagged event records to disk; monitors detector performance, electronics, and environmental status; performs online analysis and histogramming; and provides operator control of the experiment. IBM 3090 mainframe computers are used in the offline analysis of data recorded on magnetic tape.

The VAX is connected to the CAMAC network via a VAX CAMAC channel (VCC [99]) capable of a data transfer rate of 1 Mbyte/sec over a UNIBUS. The VCC communicates 
with two system crates containing 11 branch drivers which connect with 44 CAMAC crates as shown in Fig. 37. In many cases, there is a BADC in each crate which reads out the

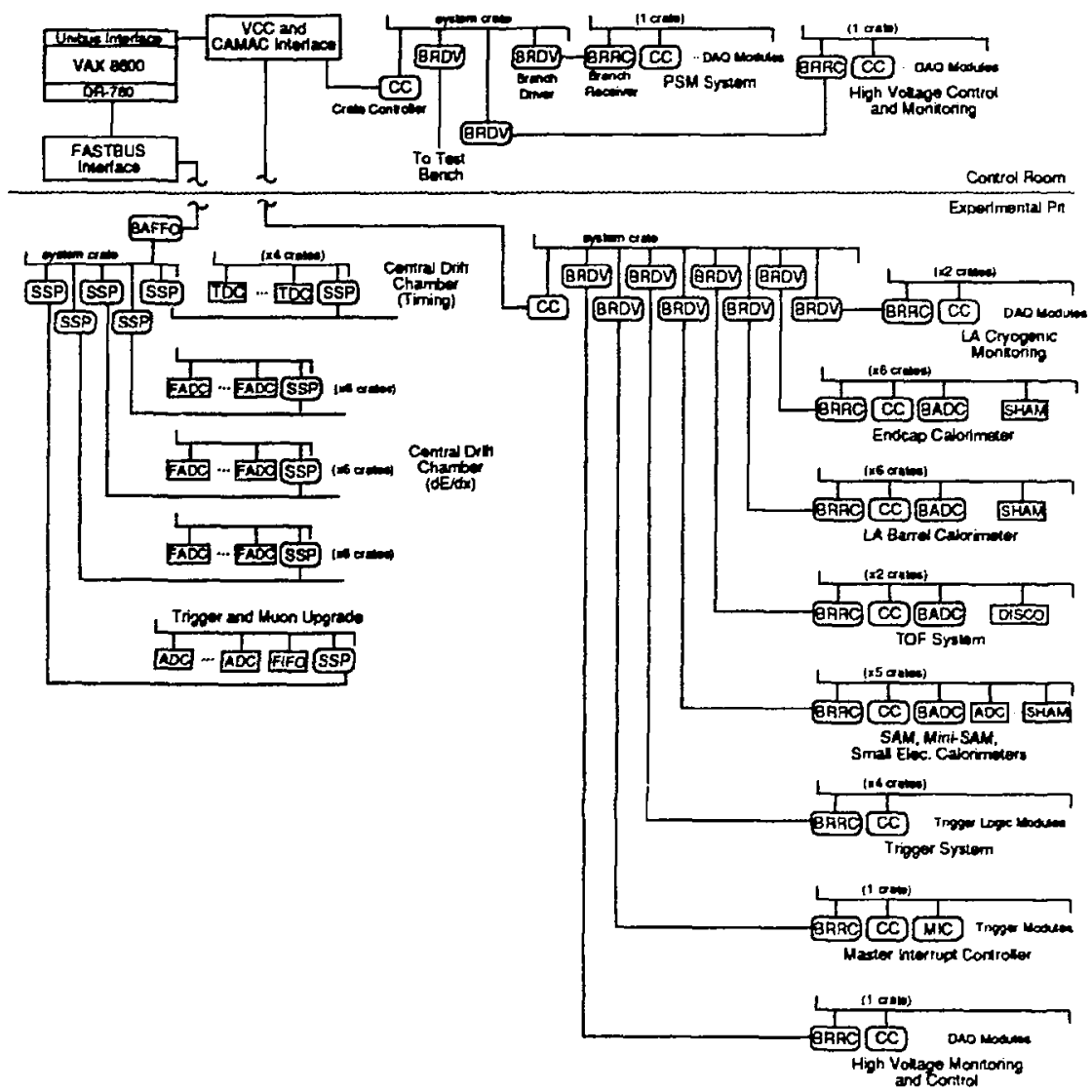

Figure 37: FASTBUS and CAMAC architecture of Mark II data acquisition system.

channels of the data acquisition modules in the crate, zero suppresses the data, and converts to physical quantities using constants stored in the BADC from previous calibration runs. 
The VCC sequentially reads out the memories of the BADCs or instrumentation modules and automatically formats the data as instructed by a database. The formatted data is put directly into the VAX memory via a direct memory access.

The FASTBUS interface is a 32-bit parallel port connecting through a simple buffer module (BAFFO) to the FASTBUS crate segment (Fig. 37). The crate segment communicates with SSPs and can transfer data to the VAX at a rate of $5.5 \mathrm{Mbytes} / \mathrm{sec}$. 1 system crate contains five system SSPs which control each of five FASTBUS cable seg ients connecting to 25 remote data acquisition FASTBUS crates [100]. Remote crate SSPs perform local data readout and processing and report to their system SSP. A master system SSP acts as an "event builder" collecting and reformatting the FASTBUS data and then interrupting the VAX when it is done.

At every beam crossing, the trigger logic is started, as well as BADC processing which typically takes 8 msec. If a trigger occurs, the CAMAC and FASTBUS systems are readout and the trigger reset when the data transfer is finished.

Deadtime in the data acquisition system occurs if the time taken to readout the CAMAC and FASTBUS systems exceeds the time between beam crossings. When machine backgrounds are high, many of the detector systems are swamped with noise hits, and the time taken to readout the correspondingly large amount of data can be long. Deadtimes of $30 \%$ are marginally acceptable, while larger deadtimes require tuning of the SLC machine to reduce machine backgrounds before the detector can acquire usable data. Deadtimes of $2-5 \%$ occur during "quiet" running. 


\section{Chapter 4}

\section{Monte Carlo Simulations}

To search for new quarks and leptons in $Z$ decays, it is necessary to simulate their production and decay properties and then compare the experimentally observed data to this simulated data. It is also important to simulate events arising from $Z$ decays to the known fundamental fermions to check for consistency of the experimental data and to choose suitable event selection criteria to distinguish new particle events if they are present.

Monte Carlo (MC) computer programs can be used to simulate particle physics processes. Probability distributions (e.g. angular or momentum distributions) predicted by theory for event production or particle decay are sampled ${ }^{\dagger}$ appropriately resulting in the generation of "fake" events. Ideally, these programs should not only give the same mean behavior as experimentally cbserved events, but also contain the same degree of event-byevent fluctuations.

The final result of the Monte Carlo event generators are the charges, particle types, and four-vectors of the final state particles of the $e^{+} e^{-}$annihilation. Using this information, the response of the Mark II detector to the particles of the event is simulated, again using Monte Carlo techniques. The simulated data is processed using the same analysis programs as applied to the experimentally observed data.

This chapter first describes the Monte Carlo models and generators that are used. The implementation of new quark and lepton processes in the framework of these generators is then discussed. Finally a brief account is rendered of the Mark II detector simulation.

\footnotetext{
'The sarnpliug is driven by soltware random number generators, which provide thr. dicte for the statistical sampling; hence the name. Mfonte Carlo.
} 


\subsection{Monte Carlo Event Generators $(Z \rightarrow q \tilde{q}, q=u, d, s, c, b)$}

Any generator producing hadrons must deal with the conversion of partons (quarks and gluons) into hadrons, and this process can be subdivided into two steps. In the first step, a parton configuration is selected using perturbative QCD results. In the second step, using phenomenological models, these partons are then allowed to fragment into hadrons. Both steps are treated differently in different MC models. Several models are considered since fine distinctions in final event shape parameters of dissimilar models lead to systematic errors in detection efficiencies and background estimates.

The parameters of the MC models were tuned using Mark II data from PEP at an $E_{\mathrm{cm}}$ of $29 \mathrm{GeV}$ [104]. If properly treated, these parameters should be valid at an $E_{\mathrm{cm}}$ of 91 $\mathrm{GeV}$ and the MC models should provide a good description of the date at these energies, as verified by measurements of hadronic event parameters at the SLC [105]. The values of important parameters used in the hadronic MC models are listed in Table 7 following the descriptions of the models.

\subsubsection{LUND (JETSET 6.3)}

The LUND (JETSET 6.3) program [101] is a pidely used Monte Carlo event genere tor. It allows the choice between two theoretical approaches to the calculation of part a distributions and the choice between several different fragmentation functions.

\section{Matrix Element}

The QCD matrix element for on-shell partons has been exactly calculated to $\mathcal{O}\left(\alpha_{s}^{2}\right)$ [102]. These calculations give the momente and directions of up to four partons. F.ithough two parton $(q \bar{q})$ states are the most common, the event generator also produces t'ree ( $q \bar{q} g$ ) and four ( $q \bar{q} q \bar{q}, q \bar{q} g g$ ) parton states. The relative production of the two, three, and four

parton states is determined by the parameter $\Lambda_{\overline{M S}}$ and a parameter $y_{\min }=m_{\min }^{2} / s$ which is the minimum scaled invariant mase two partons must have to prevent the $n$ from being combined into one.

\section{Parton Shower}

In this approach to the calculation of parton distributions, jet develc pment is perceived as being a shower process. The primary quesks are produced off-shell and decay into virtual 
partons (mostly gluons) which in turn decay. At each branching, instead of using the explicit $\mathcal{O}\left(\alpha_{s}^{2}\right)$ calculation, the matrix element is regularized by giving a mass to the gluon and summing up the leading logarithmic contributions (leading-log-approximation, LLA). The partons continue to lose virtuality on each branching until they reach a chosen cut-uff mass $Q_{0}$. Compared to the Matrix Element method, a much higher parton multiplicity is the result.

\section{Fragmentation: String Model}

In recent years, the String Model has turned out to be the mast successful and popular description [103] of jet properties at $E_{\mathrm{cm}}>25 \mathrm{GeV}$. In this case the hadrons are formed along a "string" stretched between the outgoing partons. The string tension represents the strength of the color field (growing linearly with distance) and as soon as the tension becomes large enough, the string "breakes", and the energy is converted into mass by the formation of $q \bar{q}$ pairs at the breakpoints of the string. Gluons are formed at momentum carrying bends or "kinks" in the color string. The $q \bar{q}$ pairs are then arranged into hadrons. The obeerved distribution of transverse momenta of hadrons from the quark axis $\left(p_{\perp}\right)$ is simulated by sampling from a probability distribution proportional to $\exp \left(-p_{\perp}^{2} / 2 \sigma_{q}\right)$, where $\sigma_{q}$ is an input parameter.

Both the Lund symmetric and Peterson fragmentation functions as deacribed in Section 2.2.2 are employed. When it is stated that Lund symmetric fragmentation is used, then it is applied to all five quark Alavors: $u, d, s, c$, and $b$. When it is stated that Peterson fragmentation is used, Peterson fragmentation is applied to $c$ - and b-quarks, and Lund symmetric fragmentation to the remaining light quarks.

\subsubsection{Webber (BIGWIG 4.1)}

The Webber (BIGWIG 4.1) model [106] also uses the LLA parton shower technique to produce the initial parton distribution, and uses exact $\mathcal{O}\left(\alpha_{s}\right)$ calculations to weight the momenta of the first gluon splitting. However, the implementation of the parton shower mechanism is slightly different between the LUND and Webber models in the treatment of parton mass at each step and maintenance of energy and momentum conservation. 


\section{Fragmentation: Cluster Model}

In the Webber model, the hadronization mechanism after the parton shower is described by the Cluster Model [107]. The fragmentation of the generated quarks and gluons proceeds in three steps. Each of the final gluons is forced to split into a $q \bar{q}$ pair. Pairs of quarks and anti-querks are then combined into color singlet clusters. Finally, each cluster decays isotropically (in its rest frame) into two observable hadrons, with the relative probability for different decay channels given by phase space and spin counting factors. The rate of this branching process can be calculated using perturbative QCD, the cluster formalism does not rely on fragmentation functions. The observed $p_{\perp}$ is also generated solely as a byproduct of cluster decays. A recent change to the Webber generator is the string-like fragmentation of clusters heavier than a parameter $M_{c}$, after which fragmentation via the cluster model decay is followed.

Table 7: Monte Carlo model parameters used.

\begin{tabular}{lclc}
\hline \hline Model & Parameter & Description & Value \\
\hline LUND & $\Lambda_{\text {MS }}$ & QCD Scale & $0.5 \mathrm{GeV}$ \\
(JETSET 6.3) & $y_{m i n}$ & Cutoff for combining partons & 0.015 \\
Matrix Element & $a$ & Lund symmetric fragmentation & 0.9 \\
& $b$ & Lund symmetric fragmentation & $0.7 \mathrm{GeV}^{-2}$ \\
& $\epsilon_{c}$ & Peterson frag., $c$-quark & 0.15 \\
& $\epsilon_{b}$ & Peterson frag., b-quark & 0.02 \\
& $\sigma_{q}$ & Width of $p_{\perp}^{2}$ gaussian & $0.265(\mathrm{GeV} / c)^{2}$ \\
\hline LUND & $\Lambda_{L L A}$ & QCD Scale & $0.4 \mathrm{GeV}$ \\
(JETSET 6.3) & $Q_{0}$ & Cutoff for parton evolution & $1.0 \mathrm{GeV}$ \\
Parton Shower & $a$ & Lund symmetric fragmentation & 0.45 \\
& $b$ & Lund symmetric fragmentation & $0.9 \mathrm{GeV}-2$ \\
& $\epsilon_{c}$ & Peterson frag., o-quark & 0.15 \\
& $\epsilon_{b}$ & Peterson frag., b-quark & 0.02 \\
& $\sigma_{q}$ & Width of $p_{\perp}^{2}$ gaussian & $0.23(\mathrm{GeV} / c)^{2}$ \\
\hline Webber & $\Lambda_{L L A}$ & QCD Scale & $0.20 \mathrm{GeV}$ \\
(BIGWIG 4.1) & $Q_{0}$ & Cutoff for parton evolution & $0.75 \mathrm{GeV}$ \\
& $M_{c}$ & Cluster mass cutof & $3.00 \mathrm{GeV}$ \\
\hline \hline
\end{tabular}

Quark Masses $\left(\mathrm{GeV} / c^{2}\right): m_{u}=0.325, m_{d}=0.325, m_{s}=0.5, m_{c}=1.6, m_{b}=4.98$ 


\subsection{Implementation of New Particles}

To determine detection efficiencies after event selection criteria have been imposed, it is required that Monte Carlo event simulations be made of the new quark and lepton processes. In all cases, the LUND parton shower model witb Peterson fragmentation is used as a benchmark for generating events to determine dftection efficiencies. Different models and fragmentation schemes are used to estimate systematic errors due to modelling uncertainties.

\subsection{1 $t$ and $b^{\prime}$ with Charged Current and $H^{ \pm}$Decays}

The LUND model provides for the generation of $t$ and fourth generation quarks. The event generator forms heavy hadrons from the new quarks, and in the decays of the hadrons, gluon emission effects are incorporated by the parton shower model based on the leadinglog-approximation. In the Peterson fragmentation function, the parameter $\epsilon_{\mathcal{Q}}$ for heavy quarks is given by $\epsilon_{Q}=\left(m_{0} / m_{Q}\right)^{2}$ where $m_{0}=0.70 \mathrm{GeV} / c^{2}$.

The default decky mode for heavy quarks in the LUND generator is the charged current decay mode. Slight modifications also allow the decay of heavy quarks into a $H^{ \pm}$of any mass which is kinematically accessible. The charged Higgs then decays to either $c \bar{s}$ or $\tau \bar{\nu}$, with the $c \bar{s}$ fragmentation performed by the LUND program.

The Webber MC also permits the generation of $t$-quarks, and alterations have been made to allow the generation of $b^{\prime}$-quarks.

\subsection{2 $b^{\prime}$ with FCNC Decays}

The FCNC decays of the $b^{\prime}$-quark are not normally part of the LUND event generator. Decay routines have been added to admit the decay modes $b^{\prime} \rightarrow b \gamma$ and $b^{\prime} \rightarrow b g$. The decay mode $b^{\prime} \rightarrow b \gamma$ is implemented as a two-body decay mode inside the parton shower using the leading-log-approximation; therefore, the $b^{\prime}$-quark can suffer gluon emission before the photonic decay. Another way to consider the decay is to regard the $b^{\prime}$-hadron as undergoing the decay to a photon and $b$-hadron. The case of the more simplistic treatment of a two body decay $b^{\prime} \rightarrow b \gamma$ immediately after the production of the $b^{\prime}$ (with no gluon emission before the decay) is also checked to estimate systematic errors due to modelling uncertainties.

The decay $\forall \rightarrow b g$ is treated similarly, with the gluon incorporated in the parton shower. The case of the virtual gluon decay, $b^{\prime} \rightarrow b g^{*}$, is also checked as suggested by Ref. [50], again 
to estimate the magnitude of modelling uncertainties.

\subsubsection{Leptons $L^{-}$and $\nu_{4}$ (LULEPT)}

\section{Heavy Charged Leptors}

The decay mode $Z \rightarrow L^{+} L^{-}$, including lowest order initial state radiation, is simulated using the program LULEPT [108]. Spin effects leading to spin-spin correlations of decay properties are treated properly. The allowed decay modes, branching fractions, and decay modes for $L^{-}$decay are determined by $m_{L}$ and by the difference in the mass of the $L^{-}$ and the neutrino $\nu_{L}$ to which it couples. Single-hadron decays of the $W^{*}$ are allowed. The normalization of each hadronic decay rate is adjusted relative to the purely leptonic rate to give good agreement with the measured $r$ branching fractions for the particular case of $\tau$ decay. The program is written in the LUND generator framework, which is used to produce the multihadronic final states from $W^{*} \rightarrow u \bar{d}$ or $c \bar{s}$.

\section{Dirac and Majorana Neutrinos}

The program LULEPT has been modified [109] to simulate Dirac neutrino $Z \rightarrow \nu_{4}^{D} \bar{\nu}_{4}^{D}$ pair production and ensuing $\nu_{4}$ decays, including polarization and spin-spin correlations. Decays allow mixing to the lighter generations as discussed in Section 2.4 .5 and the possibility of long lifetimes. Branching fractions and decay kinematics are calculated using an analogous procedure as for $L^{+} L^{-}$production. For small $m_{\nu_{1}}$, the invariant mass of the $W^{*}$ is small, and single-hadron final. states such as $\pi^{ \pm}, \rho^{ \pm}, a_{1}^{ \pm}, K^{ \pm}$, and $K^{* \pm}$ are possible along with the decays into $\ell \nu_{\ell}, u \bar{d}$, and co. For a given $m_{\nu_{4}}$, all the above decays are simulated and the $\nu_{4}$ branching fractions are calculated in the Monte Carlo. Given $\left|U_{\ell 4}\right|^{2}$, the $\nu_{4}$ lifetime is calculated using Eq. 52 .

Further alterations in LULEPT permit the production of Majorane neutrino pairs, $Z \rightarrow \nu_{4}^{M} \nu_{4}^{M}$, with the angular distribution described earlier in Section 2.4.4. Proper spin correlations [110], lifetimes $\left(\tau\left[\nu_{4}^{D}\right]=2 \tau\left[\nu_{4}^{M}\right]\right)$, and the possibility of same-sign isolated lepton decays are incorporated.

\subsection{Mark II Detector Simulation}

The Monte Carlo detector simulator models the response of the Mark II detector to the passage of charged and neutral particles. The four-vectors from an event generator 
Monte Carlo are followed through the subsystems of the detector allowing for the curvature of charged particles in the magnetic field. Multiple scattering, gamma conversions, bremstrahlung, nuclear interactions, and energy loss $(d E / d x)$ of particles are included according to the amount of material traversed. The deceys of unsteble charged and neutral particles within the Mark II detector are also taken into account with lifetimes sampled from appropriate exponential distributions. A library of electromagnetic showers created with the EGS4 [97] program allows rapid and fairly accurate simulation of electron and photon interactions in the calorimeter systems. Nuclear interaction effects are studied with the hadronic interaction Monte Carlo program FLUKA [111]. Computer time considerations do not permit the full simulation of nuclear interactions and the generation of secondary particles; when a nuclear inieraction occurs, the interacting particle stope at the point of interaction and disappears without generating further fragmentation particles.

The geometry of each detector subsystem is defined in software, the signals induced by charged or neutral particles in the subsystems are modelled, and the conversion of the signals into digital data is simulated. Random errors are introduced to smear simulated quantities at each stage according to measured resolutions. After all of the particles have been followed through the detector, their effects are added, and conflicts are resolved. For example, overlapping DC sense wire signals from pairs of very close tracks cannot be distinguished if they are too close to one another in time. Most of the known defects in the detector such as dead sense or proportional-tube vires, dead electronic readout channels, detection inefficiencies, etc. are included in an effort to simulate the Mark II detector as accurately as possible. The final Monte Carlo data record is then output in an identical format as the actual Mark II data.

Machine backgrounds resulting in stray particles striking the detector caused extra energy deposition and signals in the detector in addition to the signals from an actual event. To take this effect into account, the Monte Carlo raw data is "mixed" with the raw data from random beam crossings that were recorded near in time to the $Z$ decay events. 


\section{Chapter 5}

\section{Event Selection and Analysis}

This chapter describes the event selection criteria, or cuts, used in the searches for new quarks and leptons in $Z$ decays. A number of requirements are common to the analyses, and are described first. Distinctive topologies resulting from new particle production and decay outlined in Chapter 2 include:

- events containing high-momentum isolated tracks;

- events containing high-momentum isolated photons;

- spherical events; and

- events containing detached vertices.

These topologies are considered in choosing the cuts to maximize the hypothetical new particle signal, while minimizing the background signal. Using Monte Carlo events with full detector simulation, the detection efficiency for a particular new particle channel to satisfy a specific set of event selection criteria is determined. Finally, in order to reinterpret the measured width of the $Z$ to exclude $L^{-}$and $\nu_{4}$ production, it is necessary to describe the hadronic event selection criteria used in the $Z$ resonance fits and determine the efficiency for new particle events to satisfy these criteria.

The discussion of the use of these detection efficiencies to calculate the expected number of new particle events and background events will be delayed until the following chapter. Mass limits and exclusion regions from observations of the data will then be presented. 


\subsection{Gener al Event Selection}

It is desirable to first separate $Z$ decays in the data from backgrounds of two-photon processes (Fig. 38), beam-gas interactions, and beam-beampipe interactions. It is also desirable that reconstructed track and event quantities are restricted to ranges where they are simulated well by the Monte Carlo and where accurate event reconstruction is possible. A set of cuts common to a number of following analyses are presented below.

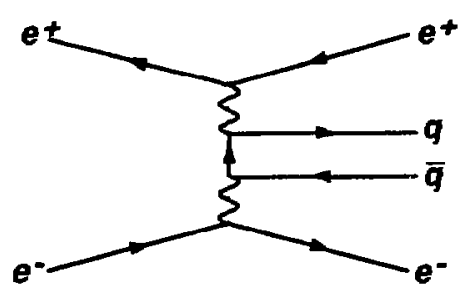

Figure 38: Feynman diagram for two-photon hadron production, a potential background. This diagram is classified as 'multi-peripheral', and is the dominant source of possible background compared to other permutations of the photons, quarks, and leptons in a two-photon diagram.

\subsubsection{Charged Track Requirements}

Reconstructed charged tracks must satisfy the following:

C1: project into the cylindrical volume defined by $r<1 \mathrm{~cm}$ and $|z|<3 \mathrm{~cm}$, centered at the IP; have

C2: $p_{x y}>150 \mathrm{MeV} / c$, where $p_{x y}$ is the transverse momentum of the track with respect to the $z$-axis; and

C3: $|\cos \theta|<0.82$, where $\theta$ is the angular direction of the track at the origin.

Cut $\mathrm{C} 1$ reduces the number of beam-gas and beam-beampipe events entering the data set. Fig. 39(a) shows the distribution of the distance of closest approach (DCA) of tracks to the $z$-axis (i.e. $r$ projection of the track) and Fig. 39(b) shows the distribution of the DCA of tracks to the $\mathrm{IP}(r=0, z=0)$ after the $\tau$ cut (i.e. $z$ projection of track). Peaks are evident centered at the IP resulting from tracks from $Z$ production and decay. Tracks due 
to background events of beam-gas and beam-beampipe interactions at large values of $z$ are also apparent.
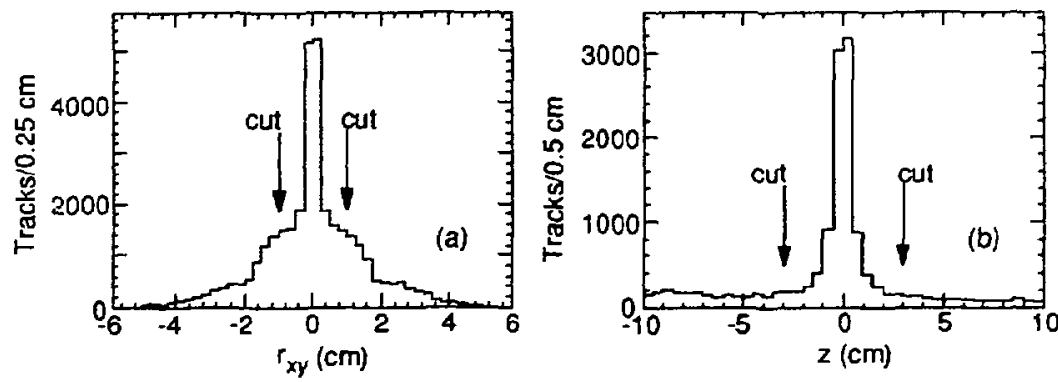

Figure 39: Distributions of (a) the two-dimensional distance of closest approsch (DCA) to the $z$ axis of changed tracks from all data triggers; and (b) DCA of tracks to the IP after the cut indicated in (a), resulting in the $z$-projection of the tracks. Cuts are indicated by arrows.
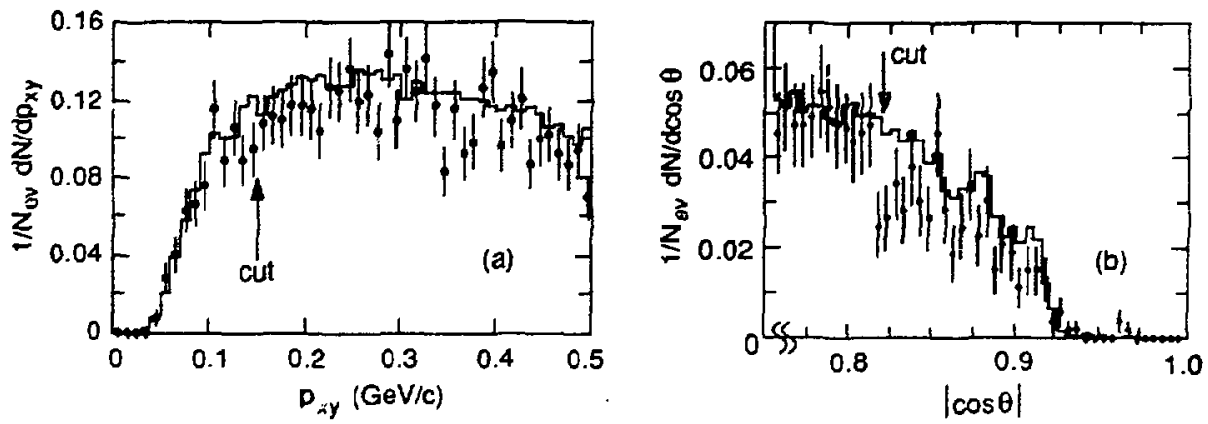

Figure 40: Distributions of (a) the transverse momenta of charged tracks with respect to the $z$-axis, and (b) the $|\cos \theta|$ values of tracks in the data (points) and from MC events (histograms). Cut values are shown by artows.

Cut C2 eliminates low momentum tracks that loop in a full circle inside the drift chamber ("loopers"). Fig. 40(a) shows that the efficiency for finding tracks with $p_{x y}>150$ $\mathrm{MeV} / \mathrm{c}$ is well understood. Cut $\mathrm{C3}$ restricts the angular range of tracks to a region where 
the MC is able to estimate track-finding efficiencies accurately as shown in Fig. 40(b). This cut is equivalent to demanding that a track pass through at least four layers of the 12 layer drift chamber before exiting. The region below $|\cos \theta|<0.7$ not shown in Fig. 40 (b) is well simulated.

\subsubsection{Neutral Shower Requirements}

A reconstructed shower or cluster not associated ${ }^{\dagger}$ with a charged track is considered for inclusion as a neutral shower from a photon. In addition, if a shower associated with a charged track has greater than twice the energy of the measured momentum of the track, the shower is dissociated from the charged track and counted as a neutral shower for the following reasons. In the case of hadronic particles, the energy deposited in the calorimeters is usually less than the particle's momentum. For electrons, we have $E \approx p$. If the shower associated with a track has $E \geq 2 p$, the extra energy is most likely due to a neutral shower overlapping the charged particle track.

Neutral showers must satisfy the following:

C4: $E_{\gamma}>1 \mathrm{GeV}$, where $E_{\gamma}$ is the energy of the reconstructed shower;

C5: $\left|\cos \theta_{\text {sh }}\right|<0.68$, where $\theta_{\text {sh }}$ is the angular position of the shower in the LA barrel calorimeter; and

C6: $0.68<\left|\cos \theta_{s h}\right|<0.85$, where $\theta_{\text {sh }}$ is the angular position of the shower in the endcap calorimeter.

Cut C4 suppresses the number of fake neutral showers from noise in the calorimeter systems and from stray particles due to machine backgrounds depositing extra energy in the calorimeter. The distribution of energy of neutral showers in the calorimeters for data and $\mathrm{MC}$ events is shown in Fig. 41. Although the region $E_{\gamma}>0.2 \mathrm{GeV}$ is well simulated after the mixing of $\mathrm{MC}$ events with data random triggers is performed, the cut is placed at $E_{\gamma}=1 \mathrm{GeV}$ to decrease the sensitivity of individual shower energy measurements to fluctuations in machine backgrounds. Cuts C5 and C6 eliminate the double counting of showers in the cverlap region between the LA and EC systems.

\footnotetext{
'Using the procedure briefly described in C'hapter 3.
} 


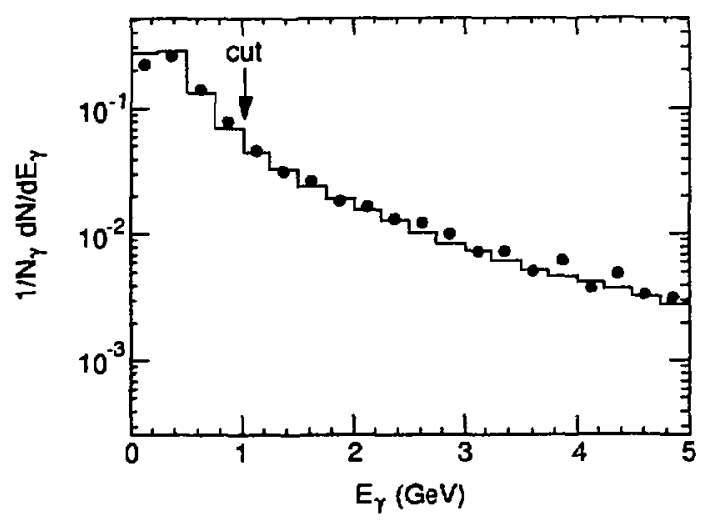

Figure 41: Distribution of the energy of neutral showers in the data (points) and from MC events (histogram). The cut is shown by the arrow.

\subsubsection{General Event Topology Requirements}

If $N_{\mathrm{ch}}$ is the number of charged tracks passing the charged track requirements, then we demand:

C7: $N_{\text {ch }}>6$.

Cut CT rejects moot cosmic rey, two-photon, beam-gas, and beam-beampipe events. It also eliminates a large fraction of lepton-pair $Z$ decay events. In almost all of the new particle scenarios considered, the final topologies usually have multiplicities greater than six, as do most normal hadronic $Z$ decay events.

Defining

we demand that

$$
E_{\text {vis }}=\sum_{\substack{\text { charged } \\ \text { tracks }}} \sqrt{|\vec{p}|^{2}+m_{\pi}^{2}}+\sum_{\substack{\text { neutral } \\ \text { showers }}} E_{\gamma}
$$

C8: $E_{\text {yis }}>0.10 E_{r m}$,

to reduce the number of two-photon events as shown in Fig. 42.

In the mejority of eeq $\bar{q}$ two-photon events, the final state electron and positron are usually emitted as very low angles and eacape unseen down the beampipe. The remaining particles visible in the detector have small total energies. 


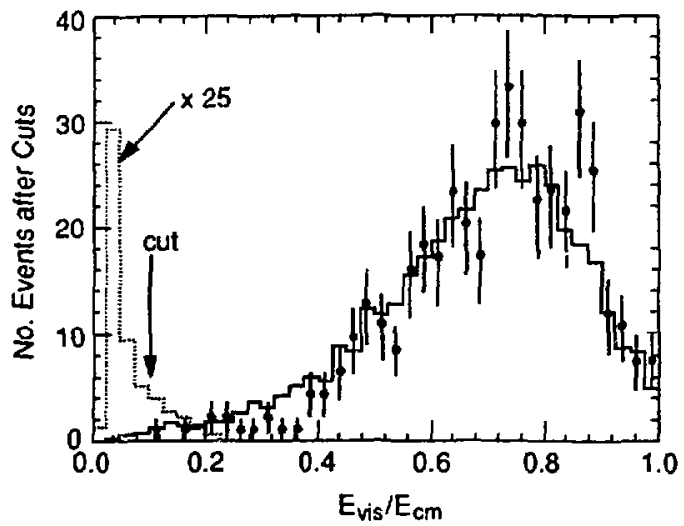

Figure 42: Distribution of the fraction $E_{\text {vis }} / E_{\mathrm{cm}}$ after cuts C1-C7 are applied for the data (points), udscb LUND MC events normalized to the data (solid histogram), and two-photon eeq $\bar{q}$ MC events [112] normalized as shown (dotted histogram).

The variable thrust $T$ is defined as:

$$
T=\max \left(\frac{\sum_{i}\left|\vec{p}_{i} \cdot \hat{n}\right|}{\sum_{j}\left|\vec{p}_{j}\right|}\right),
$$

where $\vec{p}_{i}$ is the momentum of the $i^{\text {th }}$ particle (charged and neutral), and $\hat{n}$ is a unit vector chosen to maximize $T$. The direction defined by $\hat{n}$ is called the thrust aris. We require

C9: $\left|\cos \theta_{\text {thr }}\right|<0.8$, where $\theta_{\text {thr }}$ is the polar angle of the thrust axis.

Cut C8 ensures that the event is well-contained in the detector, and events with many particles escaping undetected at low angles are avoided. Clustering algorithms (described later) are used to find the number of jets in MC (LUND Matrix Element) events. The number of jets found are compared to the number of produced partons in the MC event, and the value of cut $\mathbf{C 9}$ is chosen to provide a good match of these two quantities.

\subsubsection{Systematic Errors}

Systematic errors arising from an imperfect detector simulation are studied. $N_{\text {pass }}$ is denoted as the number of data events which pass cuts $\mathrm{C1}-\mathrm{Cg}$, and $\epsilon_{\text {pass }}$ is the efficiency to 
pass the cuts determined from MC events. The stability of the ratio $N_{\text {pass }} / \epsilon_{\text {pass }}$ is observed as cuts C1-C9 are individually varied within reasonable ranges. For example, varying the $E_{\gamma}$ cut (C4) from 0.5 to $2.0 \mathrm{GeV}$ produces changes in $N_{\text {pass }} / \epsilon_{\text {pass }}$ of $0.6 \%$. Adding the individual variations in quadrature results in an estimated $1.9 \%$ systematic error due to uncertainties in detector simulation affecting the efficiency for general event selection.

\subsection{Isolated Track Topology}

\subsubsection{Expected Signature and Background}

We consider the semileptonic decays of $t$ and $b^{\prime}$, and the leptonic decay of $\nu_{4}$. The quark charged current decay modes are $t \rightarrow b W^{*}$ and $b^{\prime} \rightarrow c W^{*}$. The probability for the semileptonic decay $W^{*} \rightarrow \ell \nu_{\ell}$ is roughly $1 / 3$, and the probability for the hadronic decay $W^{*} \rightarrow q \bar{q}^{\prime}$ is roughly $2 / 3$. Therefore, $t \bar{t}$ and $b^{\prime} \bar{b}^{\prime}$ events have the probability of approximately $5 / 9$ to contain at least one lepton from the primary decays of the heavy quarks. The charged current decay $\nu_{4} \rightarrow \ell W^{*}$ through mixing always results in at least two charged leptons in the final state of $\nu_{4} \bar{\nu}_{4}$ events, and about $8 / 9$ of the events should contain hadrons from quarks. The remaining $1 / 9$ of the $\nu_{4} \bar{\nu}_{4}$ events have a final state containing four charged leptons and missing energy; a spectacular signature, but not searched for (would fail the $N_{\text {c }}$ cut) due to its low production efficiency.

In all cases, the heavy new particles are produced nearly at rest in the laboratory frame, and the decay particles of the new particle and anti-particle tend to be relatively uncorrelated. In the heavy quark rest frame, the decay products $q$ and $\ell_{\ell}$ travel in opposite directions. Similarly, the decay products $\ell$ and $W^{*}$ of the $\nu_{4}$ travel in opposite directions in the rest frame of the $\nu_{4}$. Therefore, in a large fraction of events, a high-momentum charged lepton should be isolated relative to the other decay products.

Lepton identification is not used for the following reasons:

- detection efficiencies are reduced if the candidate track is required to be within lepton identification fiducials;

- the detection of aduitional non-leptonic one-prong decays of isolated $\tau$ leptons is made possible; and

- lepton identification and mis-identification systematic errors are avoided. 
These reasons are particularly valid considering the small data sample of $Z$ decays available. It is desirable to keep detection efficiencies as high as possible, and the small number of events prevent a detailed evaluation of lepton identification at SLC energies. Fortunately, the topology is sufficiently distinctive that the elimination of the lepton requirement does not invalidate the method.

Therefore, the search topology is an event containing at least one high-momentum isclated track.

The remaining substantial physics background after cuts $\mathbf{C l}-\mathbf{C g}$ is $Z$ decays into $q \bar{q}$ pairs of the five known flavors $(Z \rightarrow q \bar{q}, q=u, d, s, c, b)$ which we will term udscb events. Moderately isolated charged leptons can reeult from semileptonic decays of $c$ and $b$ quarks $\left(b \rightarrow c \ell^{-} \bar{\nu}_{\ell}, c \rightarrow s \ell^{+} \nu_{\ell}\right)$ and cascade decays $\left(b \rightarrow c X \rightarrow s \ell^{+} \nu_{\ell} X\right)$. It is also found that $u d s c b$ events can contain very isolated high-momentum tracks if one of the jets fluctuates down to a single charged particle. The rest of the energy of the parton after fragmentation is carried by hadronic neutral particles (e.g. $K^{0}, n$ ) which do not deposit very much energy in the electromagaetic calorimetry.

\subsubsection{Topology Criteria: $\rho$ Parameter}

\section{Thrust Cut}

The quarks in udscb events are highly relativistic, resulting in tightly collimated jets of particles, usually in a two-jet, back-to-back topology. The thrust quantity $T$ defined in Eq. 62 is 0.5 for isotropic events, and is 1.0 for an ensemble of momentum vectors in which the vectors are all either parallel or anti-parallel to a common axis. The $T$ values of $u d s c b$ events tend toward 1, and the number of udscb events with the search topology can be reduced by demanding

$\circ T<0.9$.

This cut is also found to be highly effective for eliminating events described earlier where a jet occasionally fluctuates down to a single charged track.

\section{Track Isolation}

There are a number of different ways to quantify the isolation of a track from the rest of the decay products in an event. A commonly used method is to define a cone of certain half-angle with its axis in the same direction as the candidate track, and then denuand that 
less than a specified amount of energy appear in that cone. However, this simple method does not provide a continuous variable to quantify isolation, and does not take into account the momentum of the candidate track itself. A technique without these shortcomings is the calculation of an isolation or $\rho$-parameter [113] for each track.

\section{LCLUS}

To calculate the $\rho$-parameter for tracks, it is first necessary to divide an event into $N$ clusters or jets. The clustering algorithm (LCLUS) supplied with the LUND JETSET 6.3 MC program is used. At the start of the algorithm, all particles are treated as clusters with momenta $\vec{p}_{\ell}$. The "distance" between each pair of clusters $\ell$ and $m$ is calculated using:

$$
D_{\ell m}^{2}=\frac{2\left|\vec{p}_{\ell}\right|^{2}\left|\vec{p}_{m}\right|^{2}\left(1-\cos \theta_{\ell m}\right)}{\left(\left|\vec{p}_{\ell}\right|+\left|\vec{p}_{m}\right|\right)^{2}}
$$

where $\theta_{l m}$ is the angle between the clusters. The pair of clusters with the smallest value of $D$ are "joined" (i.e. vector momenta added) if $D<d_{\text {join }}$, where $d_{\text {join }}$ is an input parameter to the algorithm. The procedure is iterated until no more clusters are joined.

\section{$\rho$-Parameter}

To calculate the $\rho$-parameter of a track in an event, the LCLUS clustering algorithm with $d_{\text {join }}=0.5 \mathrm{GeV} / \mathrm{c}$ is applied to the charged and neutral tracks of an event excluding the candidate track $i$. We then define:

$$
\rho_{i} \equiv \min _{\text {clusters } j}\left[\left(2 E_{i}\left(1-\cos \theta_{i j}\right)\right)^{1 / 2}\right]
$$

where $E_{i}$ is the track energy in $\mathrm{GeV}$, and $\theta_{i j}$ is the angle between the track and each cluster axis as shown in Fig. 43. From Fig. 64, it can be seen that it is the closest cluster to the candidate track which is considered in the calculation of $\boldsymbol{\rho}_{i}$.

High-momentum isolated tr $\mathrm{ks}$ will have large values of $\rho_{i}$. An example of an isolated track from a MC event contrining a $b^{\prime} \mathrm{CC}$ decay is shown in Fig. 44.

The distribution of $\rho$, the maximum value of $\rho_{i}$ of all charged tracks in an event, is shown in Fig. 45 for our data sample, for $u d s c b \mathrm{MC}$ events, and for a $35 \mathrm{GeV} / c^{2} b^{\prime}$-quark as an example.

The value of $d_{\text {join }}=0.5 \mathrm{GeV} / c$ is chosen to maximize the signal to noise ratio between the new fermion signal eveuts and background udseb events. This value of $d_{j o i n}$ is much smaller than the default value scaled to an $E_{\mathrm{cm}}$ of $91 \mathrm{GeV}$. A lower value of $d_{\text {join }}$ results 


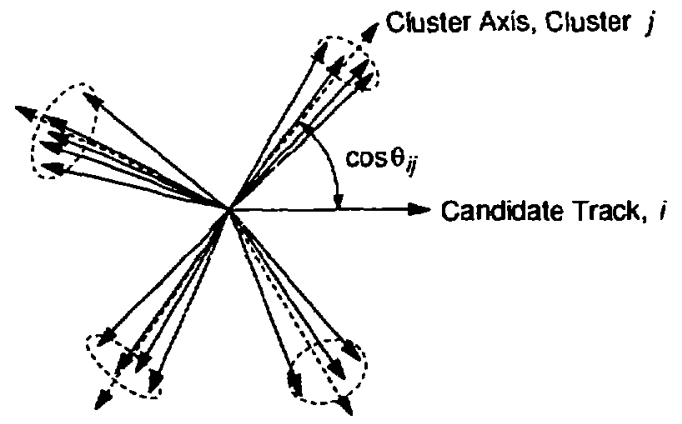

Figure 43: Schematic of the quantities used in the determination of the p-parameter.

in LCLUS finding more clusten, each containing a amaller number of charged and neutral tracks. It is found that in the background process $b \rightarrow c \ell^{-} \tilde{\nu}_{\ell}$, the potentially isolated $\ell$ can have a high momentum, resulting in the oquark forming a ralatively soft jet. It is desired that this soft jet be resolved as a separate cluster using the smaller value of $d_{j o i n}$. When using the default value of $d_{\text {join }}$, LCLUS often groups the soft $c$-jet with anot'ser cluster further away, leading to disproportionstely large values of $\rho$ for leptons from semileptonic b-quark decay.

The JADE clustering algorithm [114] was also employed to check for consistency using a different method for clustering. Input parameters to the algorithm were found which led to $\rho$ distributions similar to those obtained using the LCLUS clustering algorithm.

\subsubsection{Efficiencies}

A total number of MC signal events $N_{\text {cot }}^{M C}$ are generated and passed through the Mark II detector simulation. The number of $\mathrm{MC}$ events which pess the cuts $\mathrm{C1}-\mathrm{C}$, the thrust cut, and the isolated track $\rho$ cut is $N_{\text {cand }}^{M c}$. The detection efficiency $\epsilon_{D}$ is then simply:

$$
\epsilon_{D}=N_{\text {cand }}^{\mathrm{MC}} / N_{\text {Lot }}^{\mathrm{MC}} \text {. }
$$

Trigger efficiencies are included in $\epsilon_{D}$. The outcome of the finite size of the MC event sample is a statistical error on $\epsilon_{D}$. Using Binomial statistics [115],

$$
\Delta \epsilon_{D}=\sqrt{\epsilon_{D}\left(1-\epsilon_{D}\right) / N_{\text {Lot }}^{M C}} \text {. }
$$




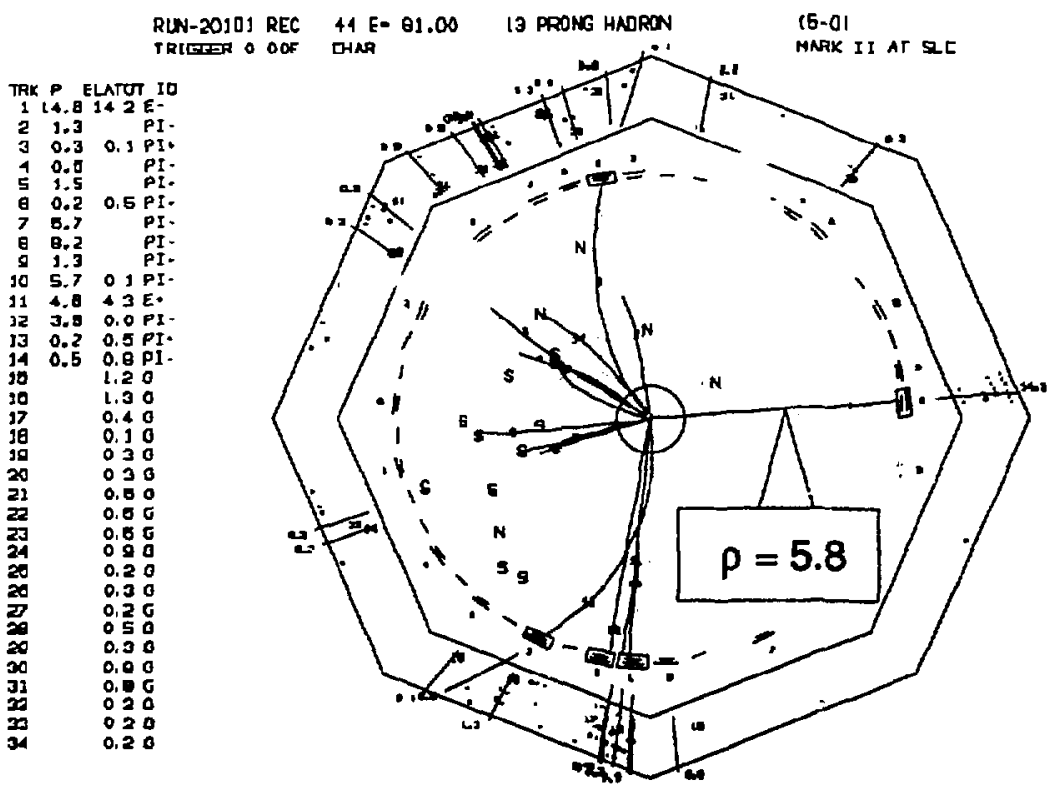

Figure 44: Example of a MC event with an isolated track. Shown is a $Z$ decay into two $35 \mathrm{GeV} / c^{2} b^{\prime}$-querke, with one of the $b^{\prime}$-quarle dacaying remileptorically into an electron with $p_{i}=5.8$. Note the simulated beckground "noive" in the detector from the "mixing" of the MC data with random beam cranings.

The uncertainty in $\epsilon_{D}$ due to $\mathrm{MC}$ statistice is given by Eq. 66 with $N_{\text {lot }}^{M C}=2000$. These errors on $\epsilon_{D}$ are omitted for clarity in the tables of this chapter.

After the charged and neutral track requirements, the effect of each event topology cut on the efficiency for signal events and udsch events to pass the criteria is shown in Table 8 for fermion mases of $35 \mathrm{GeV} / c^{2}$ as examples.

Net detection efficiencie for the signel procenes considered are shown in Table 9. Efficiencies are imell for the lower mas $t$ and $b^{\prime}$-quarks because as the quarka become more relativintic, leptow from semileptonic CC docayn are lem ieolated. Eficiencies for $v_{1} \bar{\nu}_{1}$ events are bigher then for $t \bar{t}$ and $b^{\prime} \bar{b}$ ovents because each $v_{1} \bar{\nu}_{1}$ ovent conteins at lout two leptons from $\nu_{4} \rightarrow \ell^{-} W^{*}$ and $\bar{\nu}_{1} \rightarrow \ell^{+} W^{*}$ deceya. The value of $\epsilon_{D}$ for $\nu_{4} \rightarrow e W^{*}$ is lower 


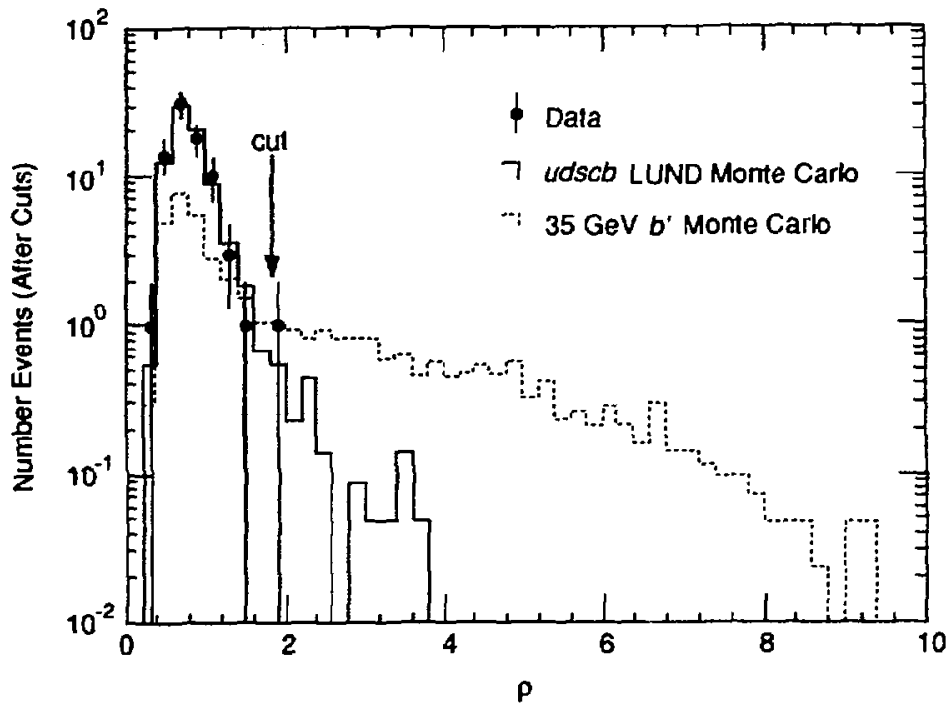

Figure 45: Maximum isolation parameter $\rho$ of all the tracks in an event for data (circles, with statistical errors), udscb QCD Monte Carlo (solid line), and a $35 \mathrm{GeV} / \mathrm{c}^{2} \boldsymbol{b}$-quark (CC decay, dashed line, normalized to data)

Table 8: Cumulative efficiencies for events passing each cut.

\begin{tabular}{|c|c|c|c|c|}
\hline Cut & $\begin{array}{c}35 \overline{\mathrm{GeV} / c^{2}} \\
t \rightarrow b W^{*} \\
\epsilon_{D}\end{array}$ & $\begin{array}{c}35 \mathrm{GeV} / c^{2} \\
W^{\prime} \rightarrow c W^{*} \\
\epsilon_{D}\end{array}$ & $\begin{array}{c}35 \mathrm{GeV} / c^{2} \\
\nu_{4} \rightarrow \mu W^{*} \\
\epsilon_{D}\end{array}$ & $\begin{array}{c}u d s c b \\
\text { LUND Shower } \\
\epsilon_{D}\end{array}$ \\
\hline$C 7: N_{c h}>6$ & 0.992 & 0.981 & 0.812 & 0.915 \\
\hline C8: $E_{\mathrm{vis}} / E_{\mathrm{cm}}>0.10$ & 0.992 & 0.981 & 0.811 & 0.912 \\
\hline C9: $\left|\cos \theta_{\mathrm{thr}}\right|<0.8$ & 0.907 & 0.890 & 0.747 & 0.788 \\
\hline$T<0.9$ & 0.882 & 0.838 & 0.703 & $0 . \overline{168}$ \\
\hline$\rho>1.8$ & 0.295 & 0.298 & 0.612 & 0.0022 \\
\hline
\end{tabular}

than for $\nu_{4} \rightarrow \mu W^{*}$ because an isolated electron has a much higher probability of radiating a photon than does an isolated $\mu$. The radiated photon weakens the electron's isolation. Detection efficiencies for $\nu_{4} \rightarrow \tau W^{*}$ are even lower due to multiprong decays of the $\tau$ and 
Table 9: Isolated track net detection efficiencies as a function of mass of a hypothetical $t$-quark, $b^{\prime}$-quark, and short-lived $(\beta \gamma c r<1 \mathrm{~cm})$ Dirac and Majorana $\nu_{4}$. The corresponding efficiency for LUND udscb MC events is $(0.22 \pm 0.10) \%$. The statistical error $\Delta \epsilon_{D} / \epsilon_{\mathrm{D}}$ is given by Eq. 66 with $N_{\text {tot }}^{\text {MC }}=2000$.

\begin{tabular}{c|cccccc}
\hline $\begin{array}{c}\text { Mass } \\
\left(\mathrm{GeV} / c^{2}\right)\end{array}$ & $\begin{array}{c}t \rightarrow b W^{*} \\
\epsilon_{D}(\%)\end{array}$ & $\begin{array}{c}b^{\prime} \rightarrow c W^{*} \\
\epsilon_{D}(\%)\end{array}$ & $\begin{array}{c}\nu_{4}^{D} \rightarrow e W^{*} \\
\epsilon_{D}(\%)\end{array}$ & $\begin{array}{c}\nu_{4}^{D} \rightarrow \mu W^{*} \\
\epsilon_{D}(\%)\end{array}$ & $\begin{array}{c}\nu_{4}^{M} \rightarrow \mu W^{*} \\
\epsilon_{D}(\%)\end{array}$ & $\begin{array}{c}\nu_{4}^{D} \rightarrow \tau W^{*} \\
\epsilon_{D}(\%)\end{array}$ \\
\hline 15 & 1.9 & 0.8 & 4.2 & 5.1 & 5.4 & 1.8 \\
20 & 6.9 & 4.3 & 21.3 & 29.2 & 28.4 & 13.8 \\
25 & 24.9 & 20.9 & 48.0 & 55.9 & 54.3 & 30.4 \\
30 & 27.5 & 28.6 & 50.2 & 58.4 & 55.9 & 31.7 \\
35 & 29.5 & 29.8 & 52.0 & 61.2 & 57.0 & 33.1 \\
40 & 28.6 & 27.8 & 55.2 & 64.2 & 60.2 & 34.9 \\
45 & 27.8 & 25.1 & 52.2 & 60.9 & 54.1 & 32.9 \\
\hline
\end{tabular}

the fact that in a single-prong $\tau$ decay, momentum is carried away by a neutrino. The

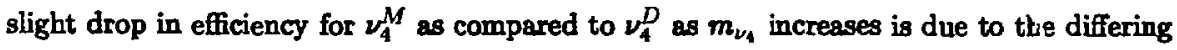
angular distributions (the Majorans distribution remains proportional to $\left(1+\cos ^{2} \theta\right)$ while the Dirac distribution becomes more isotropic) resulting in differing geometric acceptances. The observed decrease agrees with extimates made by comparing the integrals over the relevant angular range of the Msjorana and Dirac production differential cross sections. The ratio of the efficiencies for $\nu_{4}^{M} \rightarrow \mu W^{*}$ and $\nu_{4}^{D} \rightarrow \mu W^{*}$ is equal to within statistical error to the ratio of efficiencies for mixings to the other generations.

\section{Systematic Errors}

In addition to the uncertainties $(\approx 3 \%)$ from $M C$ statistics as listed in Table 9 , systematic effects on the uncertainty $\Delta \varepsilon_{D} / \epsilon_{\mathrm{D}}$ are also estimated. By varying $\mathrm{MC}$ detector simulation parameters within known errors, and finding $\epsilon_{D}$ values without the "mixing" of events with machine background noise, a systematic error of $2.5 \%\left(\Delta \epsilon_{D} / \epsilon_{D}\right)$ is assigned due to detector simulation and beam backgrounds. Theoretical uncertainties [36] in semileptonic branching ratios for $t$ and $b^{\prime}$ result in a $2.0 \%$ systematic error for $t$ and $b^{\prime}$.

There are significant systematic errors from uncertainties in quark fragmentation modelling. In the case of $t$ and $b^{\prime}$, this effect is estimated by comparing the $\epsilon_{D}$ predicted by 
using the LUND parton shower MC with Peterson fragmentation to generate the heavy quarks to that predicted by using the Monte Carlo programs:

- LUND parton shower with LUND symmetric fragmentation;

- LUND matrix element with Peterson fragmentation;

- LUND matrix element with LUND symmetric fragmentation; and the

- Webber cluster model.

Differences in predicted $\epsilon_{D}$ between the different models is found to vary with the heavy quark mass. For masses approaching the beam energy, differences are negligible, while for masses in the range $25-30 \mathrm{GeV} / c^{2}$, variations of up to $12 \%\left(\Delta \epsilon_{D} / \epsilon_{D}\right)$ for $t$ and $10 \%$ for $b^{\prime}$ are observed. A conservative approach is followed, and these maximal deviations are assigned as the systematic error due to fragmentation for all heavy quark masses. Varying the Peterson fragmentation parameters $\epsilon_{q}$ (see Eq. 35) by factors of two results in smaller differences in predicted $\epsilon_{D}$ than the differences noted between models.

In the case of $\nu_{4}$ events (generated using LULEPT and subsequent quarks fragmented by LUND), $\epsilon_{D}$ values predicted using LUND symmetric fragmentation or Peterson fragmentation are compared. Differences of up to $3 \%$ are observed due to fragraentation uncertainties. Varying fragmentation parameters $\epsilon_{c}$ and $\epsilon_{b}$ by factors of two gives differences of up to $2 \%$.

For each new heavy fermion case, individual systematic errors on $\epsilon_{D}$ are added in quadrature along with the MC statistical error.

\subsection{Isolated Photon Topology}

\subsubsection{Expected Signature and Background}

High-energy isolated photons can be the result of the FCNC decay $b \rightarrow b \gamma$. For the purpose of illustration, we first assume that $B r\left(b^{\prime} \rightarrow b \gamma\right)=100 \%$. Arbitrary decay mixtures of the $b^{\prime}$ will be considered later. A possible background is udscb events with initial or final state radiation (i.e. $q \bar{q} \boldsymbol{\gamma}$ ).

\subsubsection{Topology Criteria: $\rho_{\gamma}$ Parameter}

We again require that $T<0.9$ and now demand that there be at least one isolated photon in an event. An isolated photon is defined as a neutral shower with $\rho_{\gamma_{1}}>3.0$ where 
$\rho_{\gamma_{\mathrm{t}}}$ is defined as for a charged track. A larger $\rho_{\gamma}$ cut is required as compared to the $\rho$ cut because the calorimeters cannot resolve closely spaced $\pi^{0} \mathrm{~s}$ as well as the drift chamber can resolve closely-spaced charged pions.

The distribution of $\rho_{\gamma}$, the maximum value of $\rho_{\gamma_{i}}$ of all neutral showers in an event, is shown in Fig. 46 for our data sample, for $u d s c b$ MC events, and for a $35 \mathrm{GeV} / c^{2} b^{\prime}$-quark as an example.

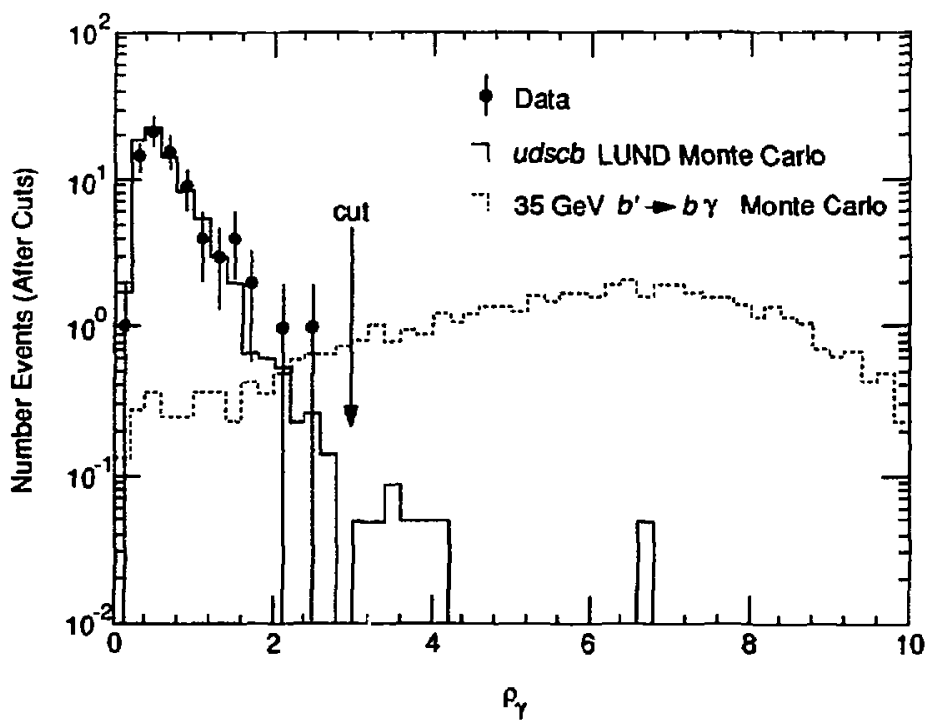

Figure 46: Maximum isolation parameter $\rho_{\gamma}$ of all the neutral showers in an event for data (circles, with statistical errors), udscb QCD Monte Carlo (solid line), and a $35 \mathrm{GeV} / c^{2} b^{\prime}$-quark (dashed line, normalized to data.)

\subsubsection{Efficiencies}

Net detection efficiencies for the process $b^{\prime} \rightarrow b \gamma$ (assuming that this is the only decay mode for the $b^{\prime}$-quark) are shown in Table 10 . Since the final state of each event contains two photons, the detection efficiency is high, and decreases at low and very high masses for similar reasons as the isolated track detection efficiencies. 
Table 10: Detection efficiencies for $b^{\prime} \rightarrow b \gamma \mathrm{MC}$ events as a function of $m_{b^{\prime}}$. The corresponding efficiency for LUND udscb MC events is $(0.082 \pm 0.030) \%$. Statistical errors are given by Eq. 66 with $N_{\text {lot }}^{\mathrm{MC}}=2000$.

\begin{tabular}{c||ccccc}
\hline \multirow{2}{*}{ Process } & \multicolumn{5}{c}{ Mass $\left(\mathrm{GeV} / c^{2}\right)$} \\
\cline { 2 - 6 } & 25 & 30 & 35 & 40 & 45 \\
\hline \hline$b^{\prime} \rightarrow b_{\gamma} \epsilon_{D}(\%)$ & 48.8 & 66.8 & 69.7 & 65.0 & 58.8 \\
\hline
\end{tabular}

\section{Systematic Errors}

Comparing values of $\epsilon_{D}$ for isolated photons using different fragmentation models, there are differences in $\epsilon_{D}$ of up to $4 \%$, depending on $m_{b^{\prime}}$. Different treatments of gluon radiation before and after the $b$ decays results in a systematic error of $3 \%$. These systematic errors are added in quadrature to the one due to machine backgrounds and detector simulation stated previously.

\subsection{Spherical Event Topology}

\subsubsection{Expected Signature and Background}

In the CC decays $t \rightarrow b W^{*}$ and $b^{\prime} \rightarrow c W^{*}$, the probability for the hadronic decay $W^{*} \rightarrow q \tilde{q}^{\prime}$ is roughly $2 / 3$. In approximately $4 / 9$ of heavy quark events, both quark and antiquark decay hadronically, and these decays result in events which tend to be spherical. This is an additional topology along with the isolated track topology, and provides another independent method to search for new heavy quarks.

However, the primary motivation to search for spherical events is the pcssibility of the decays $t \rightarrow b H^{+}, b^{\prime} \rightarrow c H^{-}$, and $\boldsymbol{b}^{\prime} \rightarrow b g$. Ensuing hadronic decays $H \rightarrow q \vec{q}^{\prime}$ lead to spherical events. The possibility of the decay $H \rightarrow \tau \nu_{\tau}$ weakens the degree of sphericity somewhat, and is also investigated. The decay mode $b^{\prime} \rightarrow$ bg results in 4 -jet events which are apt to be aplanar.

The dominant background to the above topology is multi-jet ( $>3$ jets) udscb events from multiple-bard-gluon radiation. Fortunately, the majority of udsch events bave 2-jet and 3-jet topologies since multiple-hard-gluons arise from higher order QCD processes. 


\subsubsection{Topology Criteria: Mass out of the Plane}

To quantify the shape of an event, collective variables such as sphericity, aplanarity, and thrust have been introduced. For each event a normalized momentum tensor $M_{a b}$ is defined:

$$
M_{a b} \equiv \frac{\sum_{i} p_{i a} p_{i b}}{\sum_{i} p_{i}^{2}}
$$

where $a$ and $b$ run over the three space directions, and $p_{i}$ is the momentum of particle $i$, summed over final particles $i$ in the event. $M_{a b}$ is a symmetric matrix and can be diagonalized, with unit eigenvectors $\hat{n}_{1}, \hat{n}_{2}, \hat{n}_{3}$ and eigenvalues $Q_{j}$. Sphericity and aplanarity are defined as specific combinations of the $Q_{j}$. The tails of the distributions of aphericity and aplanarity are particularly sensitive [116] to which MC fragmentation model is used because of the quadratic form of $M_{a b}$ where particles are weighted by the squares of their momenta.

Instead, a variable linear in momentum is employed. An event plane is first defined using the three eigenvectors $\hat{n}_{j}$ of $M_{a b}$. The variable of mass out of the plane $M_{\text {out }}$ is defined as:

$$
M_{\text {out }} \equiv \frac{E_{\mathrm{cm}}}{E_{\mathrm{vis}}} \frac{1}{c} \sum\left|p_{T}^{\text {out }}\right|
$$

$r_{T}^{\text {out }}$ is the momentum component out of the event plane as shown in Fig. 47, and the sum is over all charged tracks and neutral showers. Fig. 47(a) schematically illustrates a spherical event which would give a large value of $M_{\text {out, }}$ and Fig. 47 (b) shows a 3-jet $u d s c b q \bar{q} g$ event which would result in small values of $M_{\text {out }}$. Similarly, 4-jet $b^{\prime} \rightarrow b g$ events are also expected to have large values of $M_{\text {out }}$.

Figure 48 shows the distribution of $M_{\text {out }}$ for the data sample, for a $u d s c b$ QCD MC, and for the example process $b^{\prime} \rightarrow c H^{-} \rightarrow c \overline{c s}$ for a $35 \mathrm{GeV} / c^{2} b^{\prime}$-quark $\left(m_{H}=25 \mathrm{GeV} / c^{2}\right)$.

To determine efficiencies, it is required that:

- $M_{\text {out }}>20 \mathrm{GeV} / c^{2}$.

The thrust requirement included in the previous search criteria is not used.

\subsubsection{Efficiencies}

Net detection efficiencies for the signal processes considered are shown in Table 11. The efficiency for arbitrary branching ratio $B_{\ell}=\operatorname{Br}\left(H \rightarrow \tau \nu_{\tau}\right)$ of the $H^{ \pm}$is given by

$$
\epsilon_{D}\left(B_{\ell}\right)=\epsilon_{D}^{\ell \ell} B_{\ell}^{2}+2 \epsilon_{D}^{\ell h} B_{\ell}\left(1-B_{\ell}\right)+\epsilon_{D}^{h h}\left(1-B_{\ell}\right)^{2}
$$



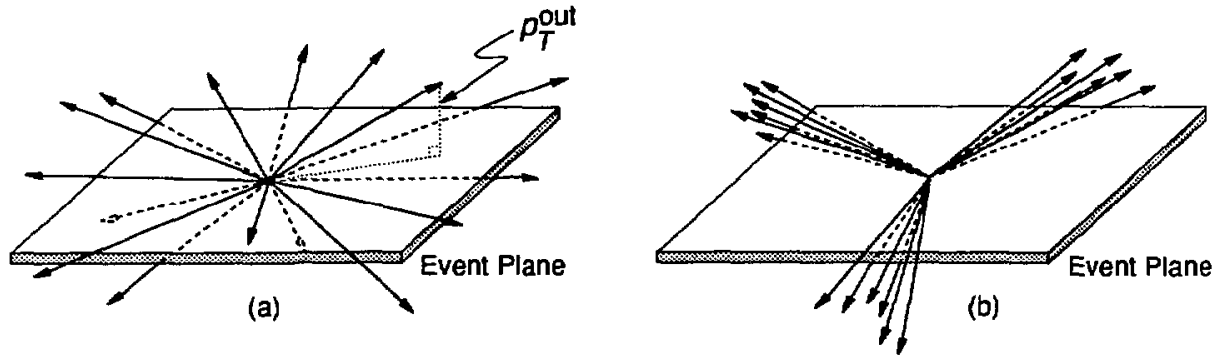

Figure 47: Schematic of $p_{T}^{\text {out }}$ as used to determine $M_{\text {out }}$. (a) shows a spherical topology typical of heavy quark hadronic and $H^{+}$decays, while (b) shows the topology of a 3-jet light quark $q \bar{q} g$ event.

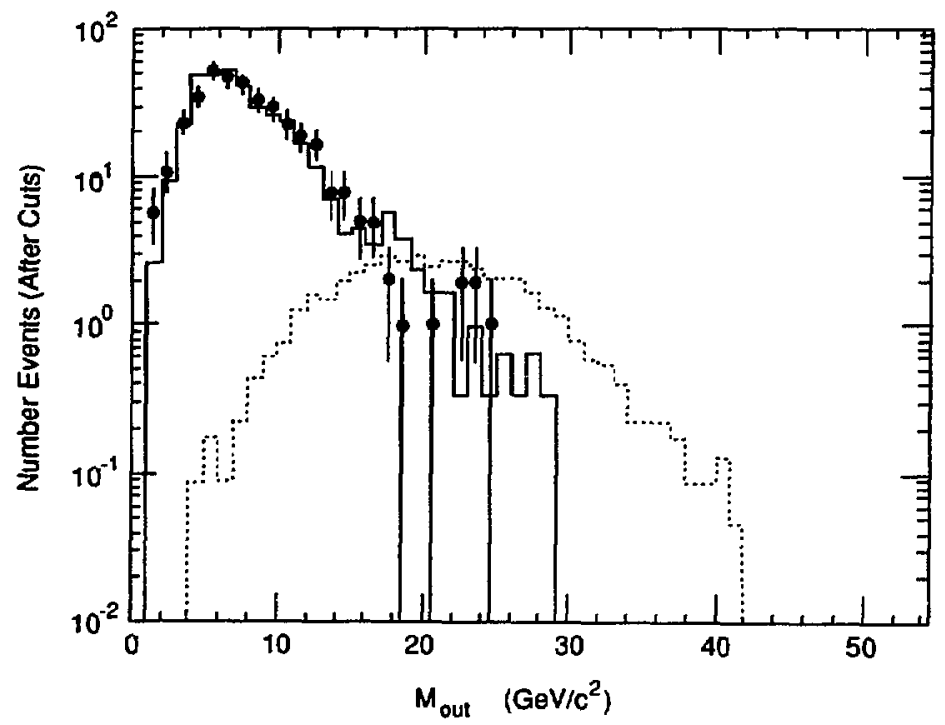

Figure 48: Distribution of mass out of the plane $M_{\text {out }}$ for data (circles, with statistical errors), udscb QCD Monte Carlo (solid line), and a $35 \mathrm{GeV} / c^{2}$ $b^{\prime}$-quark (dashed line, normalized to data.) decaying via $b^{\prime} \rightarrow \mathrm{cH}^{-} \rightarrow c \bar{c} \mathbf{s}$ $\left(m_{H}=25 \mathrm{GeV} / c^{2}\right)$. 
Table 11: $M_{\text {out }}$ detection efficiencies $\left(N_{\text {tot }}^{\text {MC }}=2000\right)$ as a function of mass of a hypothetical $t$-quark and $b^{\prime}$-quark. The corresponding efficicncy for LUND udscb MC events (parton shower, Peterson fragmentation) is $(2.0 \pm 0.3) \%$. $\epsilon_{D}^{h h}$ is the detection efficiency for both charged Higgs decaying hadronically $\left(H^{+} \rightarrow c \bar{s}, H^{-} \rightarrow \overline{c s}\right)$; $f_{D}^{c h}$ represents one $H^{ \pm}$decaying via $H \rightarrow c s$, while the other decays leptonically via $H \rightarrow \tau \nu_{\tau}$; and $\epsilon_{D}^{\ell \ell}$ is the efficiency for both Higgs decaying through $H \rightarrow \tau \nu_{\tau}$.

\begin{tabular}{c|c||cccccc}
\hline & \multicolumn{1}{|c||}{$\begin{array}{c}\text { Detection } \\
\text { Process }\end{array}$} & \multicolumn{6}{c}{ Mass $\left(\mathrm{GeV} / c^{2}\right)$} \\
\cline { 4 - 8 } & Efficiency $(\%)$ & 20 & 25 & 30 & 35 & 40 & 45 \\
\hline$t \rightarrow b W^{*}$ & $\epsilon_{D}$ & 12.5 & 26.6 & 39.8 & 52.0 & 56.1 & 45.7 \\
\hline$t \rightarrow b H^{+}$ & $\epsilon_{D}^{h h}$ & - & - & 21.2 & 65.2 & 77.1 & 70.3 \\
$\left(m_{H}=25 \mathrm{GeV} / c^{2}\right)$ & $\epsilon_{D}^{\ell h}$ & - & - & 15.8 & 46.9 & 65.4 & 57.8 \\
& $\epsilon_{D}^{\ell \ell}$ & - & - & 9.0 & 29.5 & 35.5 & 36.2 \\
\hline$b^{\prime} \rightarrow c W^{*}$ & $\epsilon_{D}$ & 5.8 & 20.7 & 32.5 & 40.3 & 47.3 & 35.2 \\
\hline$b^{\prime} \rightarrow c H^{-}$ & $\epsilon_{D}^{h h}$ & - & - & 40.1 & 60.4 & 70.3 & 58.6 \\
$\left(m_{H}=25 \mathrm{GeV} / c^{2}\right)$ & $\epsilon_{D}^{\ell h}$ & - & - & 27.5 & 46.9 & 59.0 & 46.8 \\
& $\epsilon_{D}^{\ell l}$ & - & - & 8.3 & 19.4 & 27.4 & 19.2 \\
\hline$b^{\prime} \rightarrow b g$ & $\epsilon_{D}$ & - & 12.3 & 24.5 & 32.6 & 34.8 & 21.0 \\
\hline \hline
\end{tabular}

where it is assumed that $\mathrm{Br}\left(H \rightarrow \tau \nu_{\tau}\right)+B r(H \rightarrow c)=100 \%$, and the detection efficiencies $\epsilon_{D}^{i j}$ are defined in the caption of Table 11.

Referring to Table 11, efficiencies decrease for smaller quark masses as jets become more collimated, and $\boldsymbol{M}_{\text {out }}$ decreases. Detection efficiencies also drop for very large heavy quark masses near the production threshold since the heavy quarks ace produced essentially at rest. The decay products of each heavy quark then occur back-to-back with little boosting. The two back-to-back systems define the event plane, and the decay products tend to be distributed in the plane, resulting in smaller values of $M_{\text {out }}$.

Efficiencies for the process $Q \rightarrow q H$ are given for the specific case of $m_{H}=25 \mathrm{GeV} / c^{2}$. The present mass limit for the charged Higgs is $m_{H}>19 \mathrm{GeV} / c^{2}$ from PETRA [39]. However, hadronic event shape analysis and other new particle searches at TRISTAN would have surely provided evidence for $\mathrm{H}^{+}$pair-production if is kinematically accessible. A conservative estimate of a mass limit from their data is $m_{H}>25 \mathrm{GeV} / c^{2}$. For larger values of $m_{H}$, the efficiencies for the upper mass values of $m_{Q}$ remain unchanged; but 
the efficiencies for lower $m_{Q}$ decrease faster as the kinematic limit $m_{Q}>m_{q}+m_{H}$ is approached.

\section{Systematic Errors}

Systematic errors on the $M_{\text {out }}$ detection efficiencies are estimated using the same procedure outlined in the prior section describing isolated track efficiencies. Again, differences in predicted $\epsilon_{D}$ between different fragmentation models are found to vary with heavy quark mass. Variations of up to $6 \%\left(\Delta \epsilon_{D} / \epsilon_{D}\right)$ for $t$ and $5 \%$ for $b^{\prime}$ are observed. In a conservative approach, these are the systematic errors assigned for all quark masses.

\subsection{Combined Analysis for $b^{\prime}$}

The case of $b^{\prime}$ decaying into $c W^{*}, b g$, or $b \gamma$ with any set of branching ratios is considered. If the possibility of $b^{\prime} \rightarrow \mathrm{CH}^{-}$exists, it will dominate all of the above decay modes, and is not included in this combined analysis as it has already been scrutinized.

Events are examined either for the isolated photon topology using the $\rho_{\gamma}$-parameter described earlier, or for the large $M_{\text {out }}$ topology analysis of the previous section. There are six different possibilities (along with their charge conjugate processes) with detection efficiencies labelled:

$$
\begin{array}{lc}
\epsilon^{c c}: & b^{\prime} \rightarrow c W^{*}, \overline{b^{\prime}} \rightarrow \bar{c} W^{*} \\
\epsilon^{g g}: & b^{\prime} \rightarrow b g, \overline{b^{\prime}} \rightarrow \bar{b} g \\
\epsilon^{\gamma \gamma}: & \quad b \rightarrow b \gamma, \overline{b^{\prime}} \rightarrow \bar{b} \gamma \\
\epsilon^{c \gamma}: & b^{\prime} \rightarrow c W^{*}, \overline{b^{\prime}} \rightarrow \bar{b} \gamma \\
\epsilon^{g \gamma}: & b^{\prime} \rightarrow b g, \overline{b^{\prime}} \rightarrow \bar{b} \gamma \\
\epsilon^{c g}: & b^{\prime} \rightarrow c W^{*}, \overline{b^{\prime}} \rightarrow \bar{b} g
\end{array}
$$

If we denote

$$
B_{1}=B r\left(b^{\prime} \rightarrow c W^{*}\right) ; \quad B_{2}=\frac{\Gamma\left(b^{\prime} \rightarrow b g\right)}{\Gamma\left(b^{\prime} \rightarrow b g\right)+\Gamma\left(b^{\prime} \rightarrow b \gamma\right)}
$$

and assuming

$$
B r\left(b^{\prime} \rightarrow c W^{*}\right)+B r\left(b^{\prime} \rightarrow b g\right)+B r\left(b^{\prime} \rightarrow b \gamma\right)=100 \%
$$


then the detection efficiency for $0 \leq B_{1}, B_{2} \leq 1$ is given by:

$$
\begin{aligned}
\epsilon_{D}\left(B_{1}, B_{2}\right)= & \epsilon^{c c} B_{1}^{2}+\epsilon^{g g}\left(1-B_{1}\right)^{2} B_{2}^{2}+\epsilon^{\gamma \gamma}\left(1-B_{1}\right)^{2}\left(1-B_{2}\right)^{2}+2 \epsilon^{c g} B_{1}\left(1-B_{1}\right) B_{2}+ \\
& 2 \epsilon^{c-} B_{1}\left(1-B_{1}\right)\left(1-B_{2}\right)+2 \epsilon^{g \gamma}\left(1-B_{1}\right)^{2} B_{2}\left(1-B_{2}\right) .
\end{aligned}
$$

The efficiencies shown in Table 12 are calculated by applying only one of the two analyses at a time to simplify the treatment of backgrounds. For given values of $B_{1}$ and $B_{2}$, the analysis resulting in a higher value of $\epsilon_{D}\left(B_{1}, B_{2}\right)$ is employed.

Table 12: Combined analysis detection efficiencies $\left(N_{\mathrm{tot}}^{\mathrm{MC}}=2000\right)$ as a function of $b^{\prime}$-quark mass. The detection efficiencies $\epsilon^{i j}$ are defined in the text.

\begin{tabular}{c||cccccc}
\hline \hline \multirow{1}{*||}{$\begin{array}{c}\text { Detection } \\
\text { Efficiency }(\%)\end{array}$} & \multicolumn{6}{c}{ Mass $\left(\mathrm{GeV} / c^{2}\right)$} \\
\cline { 2 - 7 } & 20 & 25 & 30 & 35 & 40 & 45 \\
\hline$M_{\text {out }}$ & 5.8 & 20.7 & 32.5 & 40.3 & 47.3 & 35.2 \\
$\epsilon^{c c}$ & - & 12.3 & 24.5 & 32.6 & 34.8 & 31.0 \\
$\epsilon^{g g}$ & 2.5 & 8.1 & 16.4 & 23.3 & 25.0 & 19.1 \\
$\epsilon^{\gamma \gamma}$ & 4.2 & 13.5 & 20.8 & 26.0 & 30.9 & 23.4 \\
$\epsilon^{c \gamma}$ & - & 10.9 & 21.5 & 28.1 & 22.3 & 27.8 \\
$\epsilon^{g \gamma}$ & 3.3 & 16.1 & 29.2 & 36.0 & 41.7 & 32.2 \\
$\epsilon^{c g}$ & & & & & & \\
\hline Isolated $\gamma$ & 13.2 & 48.8 & 66.8 & 69.7 & 65.0 & 58.8 \\
$\epsilon^{\gamma \gamma}$ & 8.6 & 34.4 & 47.1 & 49.1 & 45.8 & 41.4 \\
$\epsilon^{c \gamma}$ & 7.5 & 29.2 & 39.6 & 43.3 & 39.4 & 21.7 \\
$\epsilon^{g \gamma}$ & & & & & & \\
$\epsilon^{c c} \approx \epsilon^{g g} \approx \epsilon^{c g} \approx 0$ & & & & & &
\end{tabular}

\section{Systematic Errors}

The systematic errors and backgrounds are identical for the relevant isolated photon and $M_{\text {out }}$ analyses described in prior sections. 


\subsection{Detached Vertex Topology}

\subsubsection{Expected Signature and Background}

We consider the decay via mixing of a sequeutial neutrino $\nu_{4} \rightarrow \ell W^{*}$ as described in Section 2.4. In that section, the proportionality relation

$$
\tau\left(\nu_{4} \rightarrow \ell X\right) \propto m_{\nu_{1}}^{-5}\left|U_{\ell 4}\right|^{-2}
$$

was shown. Although the unitarity of the mixing matrix and $e-\mu, \mu-\tau$ weak universality restrict the allowed mixings [62], there is a large region where the mixing is of a value such that the decay length of the $\nu_{4}$ is experimentally observable, as in the MC event exhibited in Fig. 49. /newpage

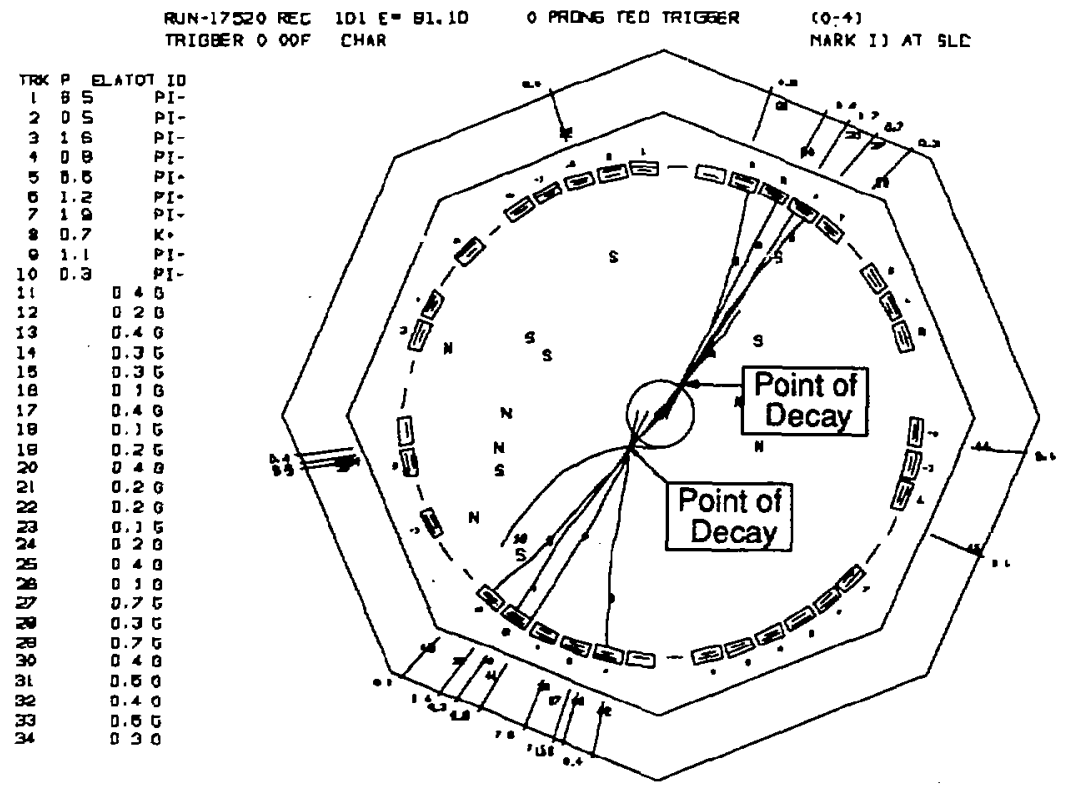

Figure 49: Example of a $M C \nu_{4} \bar{\nu}_{4}$ event $\left(m_{\nu_{4}}=35 \mathrm{GeV} / c^{2}, c r=25 \mathrm{~cm}\right)$ with detached vertices and $a$ large number of tracks with large impact parameters.

To search for this topology, one possibility is to look for events with two back-to-back 
vertices that are separated from the interaction point and with no tracks coming from the interaction point. However, if $m_{\nu_{4}}$ is large, there is frequently an ambiguity of which track or decay product belongs to which vertex, since the decay products are fairly isotropic. The probability that a particle is assigned to the wrong $\nu_{4}$ varies from $0.3 \%$ up to $20 \%$ as $m_{\nu_{4}}$ varies from 10 to $40 \mathrm{GeV} / c^{2}$ [119]. Along with large errors in vertex reconstruction, this method results in low detection efficiencies.

An alternative procedure is to examine events for the topology of a large number of tracks with large impact parameters. Impact parameter is defined as the distance of closest approach to the average beam position in the plane perpendicular to the beam axis. This method is found to result in a high detection efficiency, and is not overly sensitive to backgrounds from conventional sources.

Backgrounds which give rise to partially detached vertices and tracks with large impact parameters are charm, bottom, and strange decays; decay products undergoing nuclear interactions in detector materials such as the beampipe; misreconstructed tracks; beamgas interactions; besm-beampipe interactions; and 'noise' tracks from machine background debris striking collimators and masks.

The basic strat. $\mathrm{g}$ is to eliminate beam-gas and beam-beampipe backgrounds without using vertex criteria, and then test for events with detached vertices indicative of long decay lengths.

\subsubsection{Topology Criteria: Normalized Impact Parameter Method}

Since we are interested in tracks with large impact parameters, we do not impose cut C1 which demands that tracks project close to the IP. However, the charged track quality cuts $\mathrm{C2}$ and $\mathrm{C3}$ are retained, as are the neutral shower quality cuts $\mathrm{C4}, \mathrm{C5}$, and $\mathrm{C6}$. The remaining general event topology requirements (cuts $\mathbf{C 7}, \mathbf{C 8}$, and $\mathbf{C 9}$ ) are replaced by the requirements described below which have been optimized for the detached vertex topology.

The dominant background of beam-gas and beam-beampipe interaction events are usually forward-scattered. They also ordinarily have low multiplicity and low total visible energy. In an effort to eliminate them, we require:

$\mathrm{C7}^{\prime}: N_{\mathrm{ch}}>7$, and

$C 8^{\prime}: E_{\mathrm{vis}}>0.35 E_{\mathrm{cm}}$,

where $N_{\text {rh }}$ is the number of charged tracks satisfying cuts $C 2$ and $C 3$. In addition, the 
minimum of charged particle energy in the forward and backward hemispheres $\left(E_{f}\right.$ and $E_{b}$, respectively) with respect to the electron beam direction must be greater than $7 \%$ of $E_{\mathrm{cm}}$, i.e.,

$C g^{\prime}: \min \left(E_{f}, E_{b}\right)>0.07 E_{\mathrm{cm}}$.

To examine the effect of these cuts, and to ensure that the remaining events are free of beam-gas and beam-beampipe background, we determine the most probablc primary vertex of each event for all the data trigger events. Fig. 50(a) shows the primary vertex distribution in the plane perpendicular to the beam axis. Demanding $N_{c h}>7$ essentially eliminates all the events with primary vertices which are not at the known SLC beambeam interaction point or at the beam pipe region, leaving only the beam-beampipe hard scattering background events end events near the IP as shown in Fig. 50(b). One can also discern localized clustering of primary vertices in the regions of the wire flippers inside the beampipe. The combination of total energy requirement and the minimum forwardbackward charged energy requirement, but not the $N_{\mathrm{c} \text { s }}$ requirement, eliminates mast of the beam-beampipe interaction events, but leaves some events which are mostly misreconstructed or noise events. (Fig. 50 (c)). When all cuts are applied, there remain only events near the IP, evident in Fig. 50(d). The distributions of the $z$-coordinate of the primary vertices after each cut show similar effects. When the same cuts are applied to MC long-lived $\nu_{4} \bar{\nu}_{4}$ events, no obvious changes in the primary vertex distribution are observed.

When all requirements are applied, there remain no events with primary vertices outside a cylindrical volume centered on the IP of radius $1 \mathrm{~cm}$ and length $4 \mathrm{~cm}$ along the beam axis. The beampipe has a radius of $3.4 \mathrm{~cm}$. It is therefore conservatively estimated that there are less that 0.01 beam-gas and beam-beampipe interaction events in the 350 event sample which pass all the cuts.

\section{Normalized Impact Parameter Method}

As stated earlier, the impact parameter $b$ is the distance of closest approach in the $x-y$ plane of a charged track to the average beam pasition as shown in Fig. 51.

The average beam position for events in a particular time period is defined as the average of the fitted primary vertex positions of the hadronic events in that period. The data is divided into blocks in which there is no evidence of beam position movement of more than $200 \mu \mathrm{m}$ as determined by the SLC Final Focus beam position monitoring system. Each data block contains more than 50 hadronic events, and the typical error in the average 


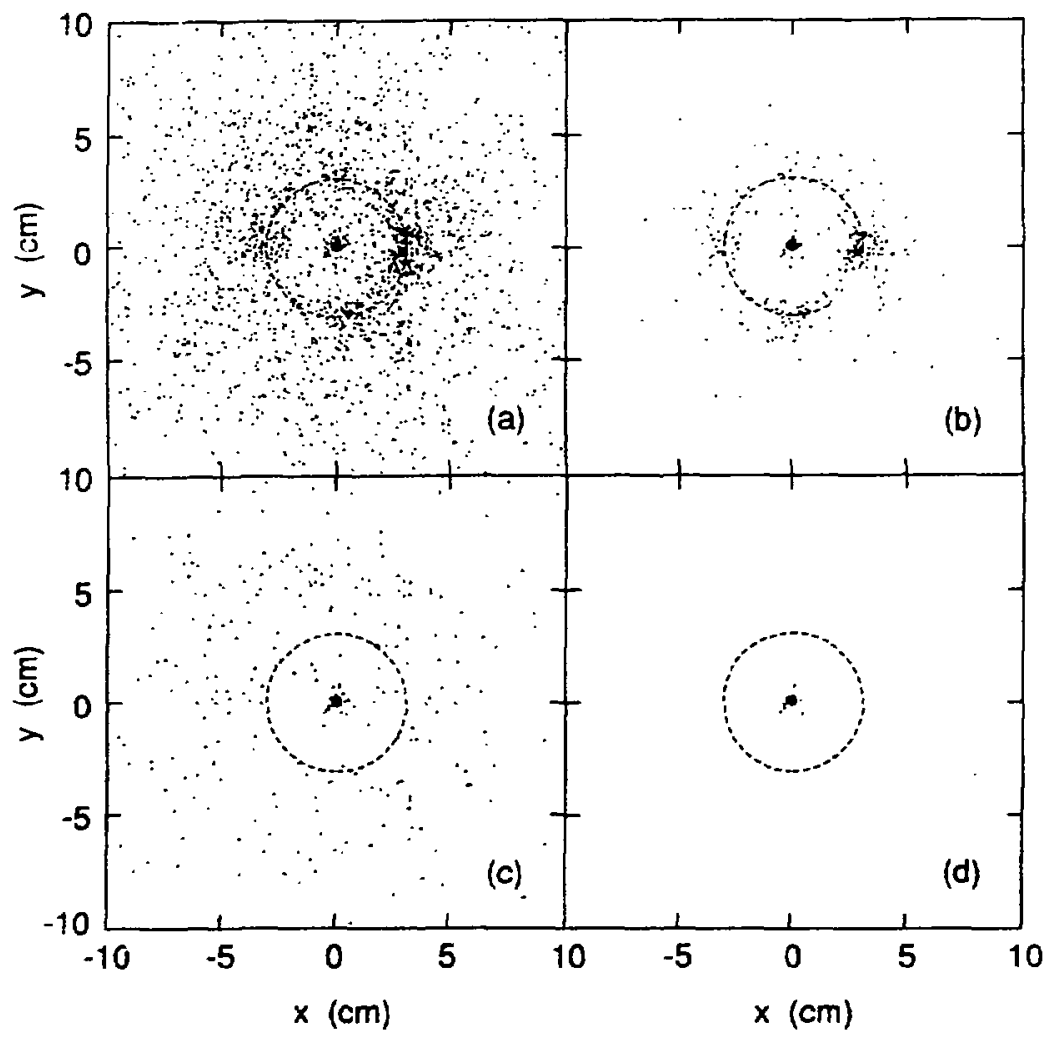

Figure 50: Distribution of the primary vertices in the $x-y$ plane for all the data trigger events after (a) only imposing track quality cuts; (b) adding the $N_{\mathrm{ch}}>7$ cut only; (c) adding the $E_{\mathrm{vis}}>0.35 E_{\mathrm{cm}}$ and $\min \left(E_{f}, E_{\mathrm{b}}\right)>0.07 E_{\mathrm{cm}}$ cuts only; and (d) after all criteria demanded. The beampipe is represented by a dashed circle.

beam position is less than $40 \mu \mathrm{m}$ in both horizontal and vertical directions. Since the SLC beam size is very small, its contribution to the uncertainty in the average beam position is negligible. Studies have also been performed on how well the measured average beam pasition tracks deliberate movements of the Final Focus quadrupole triplets. It is estimated that an upper limit on the beam position uncertainty is approximately $200 \mu \mathrm{m}$. We therefore 
conservatively assign an error $\sigma_{\mathrm{bp}}$ of $200 \mu \mathrm{m}$ to the average measured beam position for each event.

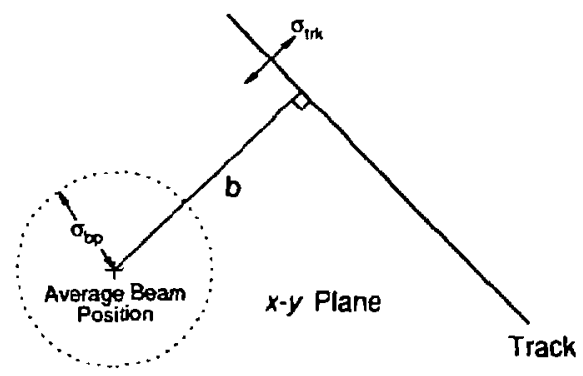

Figure 51: Definition of impact parameter and related errors.

The significance of a charged track's impact parameter is defined as the impact parameter divided by its error $\sigma_{b}$ :

$$
\sigma_{\mathrm{b}}^{2}=\sigma_{\mathrm{bp}}^{2}+\sigma_{\mathrm{trk}}^{2}
$$

where $\sigma_{\imath \mathrm{rk}}$ is the track position error perpendicular to the track trajectory determined from errcrs on the fitted track parameters. Fig. 52 shows the distribution of $\sigma_{\text {trk. }}$. The peak is due to high momentum tracks.

An event search parameter $\chi_{\text {imp }}$ is then defined as:

$$
\chi_{\text {imp }}=\frac{\text { Number of charged tracks with } b / \sigma_{b}>5.0}{\text { Number of charged tracks }} .
$$

As seen in Fig. 53, hadronic background events containing bottom, charm, and strange decays rarely yield $\chi_{\text {imp }}$ greater than 0.5 , since there are many other tracks that project to the primary vertex $x^{\dagger}$; however, many $\nu_{4} \bar{\nu}_{4}$ events with a reasonable lifetime would yield $\chi_{\text {imp }}$ values greater than 0.5 .

In Fig. 53, a clear disagrement between the data and udscb MC events is evident as a deficit of data events with very small values of $\chi_{\text {imp }}$ and an excess of data events with $\chi_{\text {imp }}$ values between 0.2 and 0.35 . This discrepancy is mainly due to the shortcomings of the MC modelling when particles in hadronic events undergo nuclear interactions in detector materials. In the MC simulation, when a nuclear interaction occurs, the incident

\footnotetext{
'The same is true for a hadronjc event with one or more particles undergoing a nuclear interaction in the beampipe or detector materials.
} 


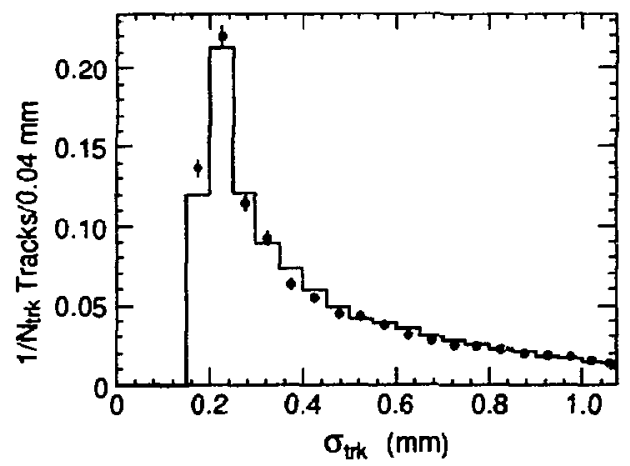

Figure 52: Distribution of $\sigma_{\text {trk }}$, the track position error perpendicular to the track trajectory, for hadronic $Z$ decay data (points) and for $u d s c b$ MC events (solid histor ram).

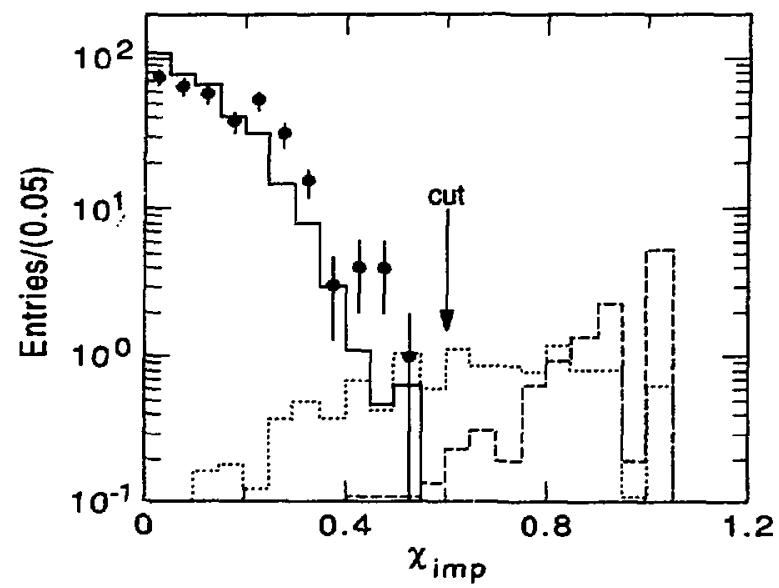

Figure 53: Distribution of $\nu_{4}$ search parameter $\chi_{\text {imp }}$ for data (solid dots with error bars), udscb $\mathrm{MC}$ events (solid line), a $35 \mathrm{GeV} / c^{2} \nu_{4}$ with a lifetime of 100 psec (dotted line, normalized to data), and a $35 \mathrm{GeV} / c^{2} \nu_{4}$ with a lifetime of 1000 psec (dashed line, normalized to data). 
particle stops at the point of interaction and disappears without generating further nuclear fragmentation particles. These secondary particles would have moderately large impact parameters. When the estimated 0.3 nuclear interactions per event is taken into account, the data and $u d s c b$ MC distributions are consistent. $Z$ decay products striking the beampipe and generating secondary particles which have moderate values of $b / \sigma_{b}$ are revealed when data events causing the excess are hand-scanned. If cut $\mathrm{Cl}$ is used to demand that tracks project close to the IP, the resulting $\chi_{\text {imp }}$ distributions for the data and MC agree weil.

To tag long-lived $\nu_{4} \bar{\nu}_{4}$ events, we demand:

$\circ \chi_{\text {imp }}>0.6$.

That is, we require that greater than $60 \%$ of the charged tracks in an event have significantly large impact parameters. This value of the cut also avoids the region of discrepancy between the data and MC.

\subsubsection{Efficiencies}

Trigger efficiencies have to be treated carefully since a long-lived $\nu_{4}$ may not decay within the detector fiducial volume defined for triggering. A full charged trigger hardware emulator program (CHGEMU) and energy-deposited trigger emulator (SSTEMUL) [117] are applied to simulated $\nu_{4} \bar{\nu}_{4}$ events. Event selection cuts are applied only to those events that would trigger our data acquisition system, and final detection efficiencies $\epsilon_{D}$ are determined. Data must pass the charged trigger emulator CHGanUL (since this emulator is slightly more restrictive than the actual charged trigger [118]) or bave satisfed the SST trigger.

As an example, for a $35 \mathrm{GeV} / c^{2} \nu_{4}$ with a $c 7$ of $1 \mathrm{~m}$, trigger efficiencies are $76 \%$ for the charged track trigger and $78 \%$ for the energy-deposited trigger, giving an overall trigger efficiency of $90 \%$; the final detection efficiency $\epsilon_{D}$ which includes the trigger efficiency is 13\%. It should be noted that the energy-deposited trigger does not employ detailed position resolution, and in general does not depend on the radius at which the $\nu_{4}$ decays as long as the $\nu_{4}$ decays before passing through the calorimeter systems.

Detection efficiencies for accessible values of $m_{\nu_{4}}$ and $\left|U_{l 4}\right|^{2}$ are listed in Table 13. Efficiencies drop for short lifetimes since the $\nu_{4}$ decays close to the IP with the consequence of small impact parameters. The impact parameter $b$ is approximately proportional to $c T$, regardless of mass, and efficiencies are roughly constant for constant $c r$. For very long mean decay lengths, one or both of the $\nu_{4}$ 's may escape the detector before decaying, leading to low trigger efficiencies. Decays in the last few layers of the drift chamber result in low track 
Table 13: Normalized impact parameter detection efficiencies $\left(N_{\text {tot }}^{\mathrm{MC}}=2000\right)$ for $\nu_{4}^{D} \rightarrow e W^{*}$. The corresponding efficiency for LUND $u d s c b$ MC events (parton shower, Peterson fragmentation) is less than $0.05 \%$ (95\% CL).

\begin{tabular}{|c|c|c|c|c|c|}
\hline \multirow{2}{*}{$\begin{array}{c}\text { Mass } \\
m_{\nu_{4}} \\
\left(\mathrm{GeV} / \mathrm{c}^{2}\right)\end{array}$} & \multirow{2}{*}{$\begin{array}{c}\text { Miving Matrix } \\
\text { Element } \\
\left|U_{e 4}\right|^{2} \\
\end{array}$} & \multicolumn{2}{|c|}{ Lifetíme } & \multirow{2}{*}{$\begin{array}{c}\text { Mean Decay Length } \\
\langle l\rangle=\beta_{\gamma c r} \\
(\mathrm{~cm})\end{array}$} & \multirow{2}{*}{$\begin{array}{c}\text { Detection } \\
\text { Efficiency } \\
\epsilon_{D}(\%)\end{array}$} \\
\hline & & $\begin{array}{c}\tau \\
(\mathrm{psec})\end{array}$ & $\begin{array}{c}c t \\
(\mathrm{~cm})\end{array}$ & & \\
\hline \multirow[t]{3}{*}{10} & $1.69 \times 10^{-7}$ & 16.6 & 0.50 & 2.2 & 14.5 \\
\hline & $2.03 \times 10^{-6}$ & 200. & 6.0 & 26.6 & 37.0 \\
\hline & $1.69 \times 10^{-8}$ & 2000 & 60. & 266 & 19.1 \\
\hline \multirow[t]{3}{*}{20} & $6.14 \times 10^{-8}$ & 16.6 & 0.50 & 1.0 & 19.0 \\
\hline & $5.10 \times 10^{-9}$ & 200. & 6.0 & 12.3 & 46.6 \\
\hline & $2.45 \times 10^{-10}$ & 4166 & 125 & 255. & 4.6 \\
\hline 30 & $6.70 \times 10^{-10}$ & 200. & 6.0 & 6.8 & 49.4 \\
\hline \multirow[t]{7}{*}{35} & $6.15 \times 10^{-9}$ & 10. & 0.3 & 0.25 & 5.0 \\
\hline & $6.15 \times 10^{-10}$ & 100. & 3.0 & 2.5 & 43.1 \\
\hline & $3.07 \times 10^{-10}$ & 200. & 6.0 & 5.0 & 43.5 \\
\hline & $6.15 \times 10^{-11}$ & 1000 & 30. & 25. & 41.1 \\
\hline & $3.07 \times 10^{-11}$ & 2000. & 60. & 50. & 26.7 \\
\hline & $2.05 \times 10^{-11}$ & 3000 & 90. & 75. & 18.7 \\
\hline & $1.54 \times 10^{-11}$ & 4000 & 120. & 100. & 13.3 \\
\hline \multirow[t]{3}{*}{40} & $8.08 \times 10^{-10}$ & 39.0 & 1.2 & 0.63 & 18.9 \\
\hline & $1.57 \times 10^{-10}$ & 200. & 6.0 & 3.2 & 47.3 \\
\hline & $1.44 \times 10^{-11}$ & 2185. & 65.5 & 35.5 & 35.5 \\
\hline 45 & $8.75 \times 10^{-11}$ & 200. & 6.0 & 0.9 & 31.6 \\
\hline
\end{tabular}

reconstruction efficiencies. The trigger, multiplicity, and visible energy cut efficiencies are more dependent on the mean decay length $\langle l\rangle$.

It is found that detection efficiencies are equal to one another within statistical errors for the different decay modes $\nu_{4} \rightarrow e W^{*}$ and $\nu_{4} \rightarrow \mu W^{*}$, and are approximately $10 \%$ lower for $\nu_{4} \rightarrow \tau W^{*}$. In the case of $\nu_{4} \rightarrow \tau W^{*}$, multi-prong decays of the $\tau^{ \pm}$tend to give more tracks with large impact parameters, and the $\chi_{\text {imp }}$ cut is more efficient; however, the presence of two more neutrincs in each event from the $\tau^{ \pm}$decays result in a lower efficiency to satisfy the visible energy requirement $\mathbf{C} 7^{\prime}$.

The measured detection efficiencies for the $\chi_{\text {imp }}$ analysis applied to simulated Majorana 
neutrinos are found to be equal, within MC statistical errors, to those for Dirac neutrinos even for high mass values. This analysis is less sensitive to the effect of differing $\nu_{4}^{D}$ and $\nu_{4}^{M}$ angular distributions because a large number of tracks in each event is considered as compared to the short-lived $\nu_{4}$ isolated track analysis. The differences in angular acceptance are therefore 'smeared out'.

\subsubsection{Systematic Errors}

Inefficiencies in the triggering system are estimated by comparing the relative efficiencies of redundant trigger components. Parameters in the trigger emulators are varied within their uncertainties to determine systematic errors in $\epsilon_{D}$ due to the trigger simulation. In particular, the performance of a number of "trigger cards" used to interface with the TDC FASTBUS backplane and provide the trigger logic "hit" signal for each DC cell were at times inoperative. During running, these intermittent modules were moved to the outer two layers of the DC to reduce their effect on the overall charged trigger efficiency. This is taken into account as well as the individual cell trigger efficiencies in the estimation of a $3.5 \%\left(\Delta \epsilon_{D} / \epsilon_{D}\right)$ systematic error due to inexact modelling of the two trigger systems. Uncertainties in calorimeter energy scales, momentum resolution, and multiple scattering effects (the latter two affecting $\sigma_{b}$ ) contributes a further $3.2 \%$ systematic error due to the detector simulation.

Track-finding and reconstruction efficiencies for tracks passing only through the outer layers of the DC are studied to check the detector simulation for $\nu_{4}$ decays occurring at large radii. MC and data tracks passing through all 12 layers of the DC are first selected. Raw data from the inner first layer is removed, the real and simulated data is again passed through the track-finding and reconstruction software, and the tracking efficiency is recalculated. This process is then repeated by removing information from the inner two layers, and so on. The comparison between data and MC tracks is shown in Fig. 54. Since at least three segments are required to form a track, the tracking efficiency in Fig. 54 is zero when the raw data from the inner 10 of the 12 layers of the drift chamber is removed. Note that the tracking efficiency is still $>50 \%$ for particles which leave tracking information only in the outer three layers of the DC.

A comparison of tracking efficiency between the data and $\mathrm{MC}$ for tracks with large impact parameters is also made. In the data, cosmic ray events are isolated from beamcrossing events to provide a sample of large impact parameter tracks. Signals in collinear 


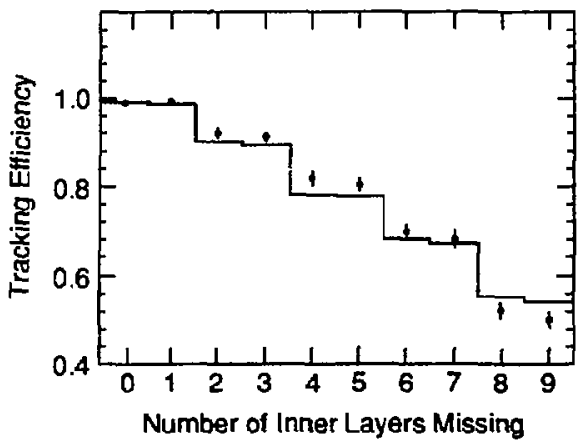

Figure 54: Tracking efficiency as a function of number of drift chamber inner layers not containing tracking information (see text) for data (dots, with statistical errors) and $u d s c b$ MC events (solid histogram) for comparison.

time-of-flight counters indicate the passage of a cosmic ray muon through the DC, and tracking efficiencies of the cosmic ray tracks are determined as a function of impact parameter. These are compared to efficiencies determined from long-lived $\nu_{4} \mathrm{MC}$ events.

As a result of these tracking studies, a $10 \% \Delta \epsilon_{D} / \epsilon_{D}$ systematic error is assigned due to tracking uncertainties for tracks with large impact parameters originating from decay vertices at large radii.

Finally, different fragmentation models are used for the treatment of the hadronic decays of the $W^{*}$ in the decay $\nu_{4} \rightarrow \ell W^{*}$, leading to the assignment of a $1.5 \%$ systematic error on $\epsilon_{D}$ from fragmentation simulation uncertainties.

\subsection{Mass Limits from Measurements of the $Z$ Resonance}

The presence of new particles from $Z$ decay can be detected in the increase of the width of the $Z$ resonance above Standard Model 3-generation, 5-quark expectations. From measurements of the $Z$ resonance, an upper limit on the number of massless neutrino species $N_{\nu}<n_{95 \%}$ at the $95 \%$ confidence level (CL) can be determined. This result can be reinterpreted to find mass limits for a stable $\nu_{4}$, an unstable $\nu_{4}$, and a heavy charged lepton $L^{-}$.

As shown earlier, the visible $Z$ cross section $\sigma_{Z}$ can be represented by a relativistic 
Breit- Wigner:

$$
\sigma_{Z}=\frac{12 \pi}{M_{Z}^{2}} \frac{s \Gamma_{e} \Gamma_{\text {vis }}}{\left(s-M_{Z}^{2}\right)^{2}+s^{2} \Gamma_{Z}^{2} / M_{Z}^{2}}(1+\delta),
$$

where $\Gamma_{e}$ is the partial width into electrons, $\Gamma_{\text {vis }}$ is the partial widths for $Z$ decays visible in the detector, $\Gamma_{Z}$ is the $Z$ total width, and $\delta$ is the substantial correction due to initial state radiation. An accurate analytical approximation [120] that includes radiative corrections can be written as a function of four input parameters:

$$
\sigma_{Z}=\sigma_{Z}\left(\sigma_{\text {peak }}, M_{Z}, \Gamma_{Z}, s\right)
$$

where $\sigma_{\text {peak }}$ is given by:

$$
\sigma_{\text {peak }}=\frac{12 \pi}{M_{Z}^{2}} \frac{\Gamma_{e} \Gamma_{\text {vis }}}{\Gamma_{Z}^{2}},
$$

for $s=M_{Z}^{2}$. A fit is performed minimizing the differences between the observed and predicted cross sections at a number of scan points of different center-of-mass energies (i.e. different $s$ ). The details of the fitting procedure can be found in Ref. [66].

To determine the invisible width (represented by $N_{\nu} \Gamma_{\nu}$, where $\Gamma_{\nu}$ is the partial width into a pair of massless neutrinos), the total width is set to:

$$
\Gamma_{Z}=\Gamma_{u d s c b}+\Gamma_{e}+\Gamma_{\mu}+\Gamma_{\tau}+N_{\nu} \Gamma_{\nu}
$$

where the partial widths are given in Section 2.1.3. The parameters $N_{\nu}$ and $M_{Z}$ are allowed to vary in the fit, and $\Gamma_{\text {vis }}$ is constrained to its Standard Model value with no new particles. In this manner, the upper limit on the parameter $N_{\nu}<n_{95} \%$ is determined.

\subsubsection{Stable $\nu_{4}$}

A stable $\nu_{4}$ would contribute only to the invisible $Z$ width, adding a fraction of a massless neutrino width, the fraction depending upon the mass of the new neutrino. Using the measured upper limit on $N_{\nu}$, a mass limit can be easily derived as described in the next chapter.

An unstable $\nu_{4}$ could lead to visible decays in the detector as determined by visible event selection criteria.

\subsubsection{Visible Event Selection Criteria}

Visible event criteria are used to define "events visible in the detector" and to define the numerical value of $\Gamma_{\text {vis. }}$ Hadronic $(u d s c b) Z$ events are selected as visible events. In 
addition, $\mu$ - and $\tau$-pair $Z$ events with thrust axes in the angular range $\left|\cos \theta_{\mathrm{thr}}\right|<0.65$ (i.e. decay products are required to pass through the LA barrel calorimeter) are included as visible events. For perfect event selection, the visible width is then

$$
\Gamma_{v i s}=\Gamma_{u d s c b}+0.556\left(\Gamma_{\mu}+\Gamma_{\tau}\right)
$$

The 0.556 factor is simply the integral of $d \sigma_{\mu \mu(\tau \tau)} / d \cos \theta$ from $\cos \theta=-0.65$ to $\cos \theta=0.65$.

In the fitting of the data to the theoretical line shape, hadronic, $\mu$-pair, and $r$-pair events need to be counted separately. For our purpose, we only need to find the efficiency for exotic events to contribute to the visible width. Visible event selection criteria are:

Reconstructed charged tracks must satisfy the following (nomenclature defined earlier):

V1: project into the cylindrical volume defined by $r<1 \mathrm{~cm}$ and $|z|<3 \mathrm{~cm}$, centered at the IP; have

V2: $\boldsymbol{p}_{x y}>110 \mathrm{MeV} / c$; and

V3: $|\cos \theta|<0.92$.

A reconstructed shower or cluster is considered for inclusion as a neutral shower whether it is associated with a charged track or not. Neutral showers must then satisfy the following:

V4: $E_{r}>1 \mathrm{GeV}$;

V5: $\left|\cos \theta_{\mathrm{sh}}\right|<0.68$, where $\theta_{\mathrm{sh}}$ is the angular position of the shower in the LA barrel calorimeter; and

V6: $0.68<\left|\cos \theta_{s h}\right|<0.85$, where $\theta_{s h}$ is the angular position of the shower in the endcap calorimeter.

Events must satisfy:

V7: $\min \left(E_{f}, E_{b}\right)>0.05 E_{\mathrm{cm}}$.

Two multiplicity cases are considered:

VBa: $N_{r h}>2$.

If R1: $2 \leq N_{\text {ch }} \leq 6$,

R2: $T>0.95$, and 
R3: $\left|\cos \theta_{1, j r}\right|>0.65$, then reject as a $\tau$-pair event outside the fiducial volume;

otherwise accept the event (hadronic or $\tau$-pair vithin the fiducial volume).

V8b: $N_{\mathrm{ch}}=2$.

If L1: $T>0.95$,

L2: $E_{\mathrm{cal}} / E_{\mathrm{cm}}<0.8$ ( $E_{\mathrm{cal}}$ is the total energy in the LA barrel calorimeter), and

L3: $\left|\cos \theta_{\text {thr }}\right|<0.65$, then accept the event ( $\mu$-pair or $\tau$-pair event within the fiducial volume).

The efficiency for hadronic events to pøss these visible event selection criteria is $(95.4 \pm$ $0.8) \%$. The efficiencies for $\tau$-pairs and $\mu$-pairs with $\left|\cos \theta_{\text {thr }}\right|<0.65$ is $(95 \pm 2) \%$ and $(97 \pm 2) \%$, respectively. The overall efficiency for Standard Model events (no new particles) to pass these selection criteria is $\epsilon_{\text {vis }}=(95.4 \pm 0.8) \%$.

\subsubsection{Unstable $\nu_{4}$}

We first assume that the neutrinos of the first three generations are massless. Secondly, when the possibility of a massive $\nu_{4}$ is considered, we assume that it is the only new particle with mass less than $M_{Z} / 2$. If $\nu_{4}$ is unstable, it will begin to contribute to the visible width and total width of the $Z$ :

$$
\begin{aligned}
\Gamma_{\mathrm{vis}} & =\Gamma_{\mathrm{vis}}^{0}+\eta_{\mathrm{vis}} \Gamma_{\nu_{4}}, \\
\Gamma_{Z} & =\Gamma_{Z}^{0}+\Gamma_{\nu_{4}},
\end{aligned}
$$

where $\Gamma_{\text {vis }}^{0}=\epsilon_{\text {vis }}\left[\Gamma_{u d s c b}+0.556\left(\Gamma_{\mu}+\Gamma_{\tau}\right)\right]$ is the visible width predicted by tive 3 -generation, 5-quark Standard Model for decays in the fiducial volume (after selection cuts), $\eta_{\text {vis }}$ is the efficiency for $\nu_{4} \bar{\nu}_{4}$ events to satisfy the visible event selection criteria, $\Gamma_{\nu_{4}}$ (a function of $\left.m_{\nu_{4}}\right)$ is the partial width for $Z$ decay into $\nu_{4} \bar{\nu}_{4}$, and

$$
\Gamma_{Z}^{0}=\Gamma_{u d s c b}+\Gamma_{e}+\Gamma_{\mu}+\Gamma_{\tau}+3 \Gamma_{\nu} .
$$

From the relation Eq. 77 for $\sigma_{\text {peak }}$, the region where the relation:

$$
\frac{\Gamma_{\mathrm{vis}}}{\Gamma_{Z}^{2}}=\frac{\Gamma_{\mathrm{vis}}^{0}+\eta_{\mathrm{vis}} \Gamma_{\nu_{4}}}{\left(\Gamma_{Z}^{0}+\Gamma_{\nu_{4}}\right)^{2}}<\frac{\Gamma_{\mathrm{vis}}^{0}}{\left(\Gamma_{Z}^{0}+\left(n_{95 \%}-3\right) \Gamma_{\nu}\right)^{2}}
$$

is true is excluded at $95 \% \mathrm{CL}$. 


\section{Efficiencies}

The efficiency $\eta_{\text {vis }}$ for $\nu_{4} \bar{\nu}_{4}$ events to pass the visible width cuts V1-V8 are measured using MC data sets with different values of $m_{\nu_{4}}$ and $\left|U_{\ell 4}\right|^{2}$. As in the detached vertex analysis, a full emulation of both trigger systems is included to properly simulate the detector response to long-lived $\nu_{4}$ decays.

Typical measured efficiencies for a $15 \mathrm{GeV} / c^{2} \nu_{4}$ are shown as a function of lifetime of the $\nu_{4}$ (which is determined by the value of $\left|U_{\ell 4}\right|^{2}$ input to the $\mathrm{MC}$ generator) in Fig. 55. The efficiency drops as the lifetime becomes longer as fewer tracks and events satisfy the visible event criteria. Values of $\eta_{\text {vis }}$ for Dirac unstable neutrinos are compiled in Table 14.

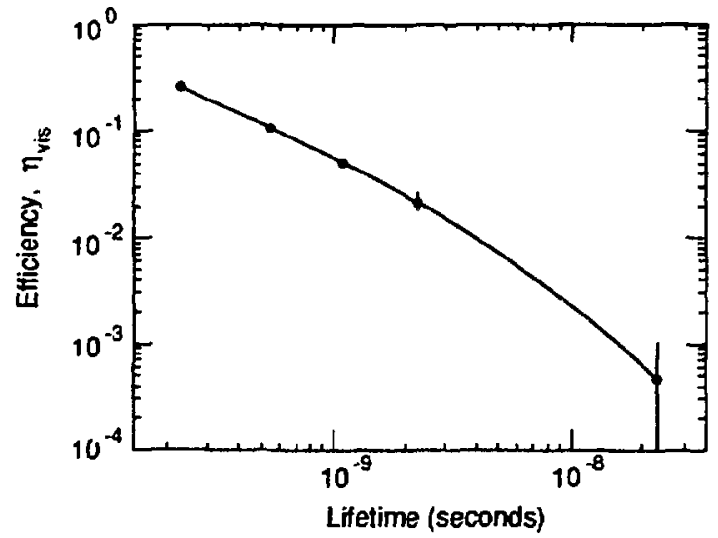

Figure 55: Measured efficiency $\eta_{\text {vis }}$ for a $15 \mathrm{GeV} / c^{2} \nu_{4}$ as a function of the lifetime of the $\nu_{4}$. Errors are from MC statistics.

Detection efficiencies for $\nu_{4}^{D} \rightarrow \mathrm{eW}^{*}$ are equal to within statistical errors to efficiencies for $\nu_{4}^{M} \rightarrow \mathrm{eW}$. The Dirac and Majorana neutrino angular distributions are much the same for $m_{\nu_{4}}<20 \mathrm{GeV} / c^{2}$.

By varying cuts V1-V8 within reasonable ranges, a systematic error of $1.4 \%$ on visible event selection is estimated. Remaining systematic errors on $\epsilon_{D}$ are very similar to those outlined for the large impect parameter analysis. 
Table 14: Efficiencies for Dirac $\nu_{4} \bar{\nu}_{1}$ events to pass visible event criteria. Statistical errors on $\eta_{\text {vis }}$ are given by $N_{\mathrm{tot}}^{\mathrm{MC}}=2000$.

\begin{tabular}{cc||cc||cc}
\hline \hline $\begin{array}{c}m_{\nu_{4}} \\
\left(\mathrm{GeV} / c^{2}\right.\end{array}$ & $\begin{array}{c}\text { Lifetime } \\
(\mathrm{sec})\end{array}$ & \multicolumn{2}{c||}{$\nu_{4}^{D} \rightarrow \mu W^{*}$} & \multicolumn{2}{c}{$\nu_{4}^{D} \rightarrow \tau W^{*}$} \\
\hline \hline 3.0 & $0.32 \times 10^{-11}$ & $0.63 \times 10^{-2}$ & 43.4 & $0.10 \times 10^{0}$ & 81.7 \\
& $0.32 \times 10^{-10}$ & $0.63 \times 10^{-3}$ & 4.3 & $0.10 \times 10^{-1}$ & 61.2 \\
& $0.32 \times 10^{-9}$ & $0.63 \times 10^{-4}$ & 0.5 & $0.10 \times 10^{-2}$ & 6.1 \\
\hline 5.0 & $0.35 \times 10^{-10}$ & $0.36 \times 10^{-4}$ & 34.6 & $0.10 \times 10^{-3}$ & 36.5 \\
& $0.35 \times 10^{-9}$ & $0.36 \times 10^{-5}$ & 2.5 & $0.10 \times 10^{-4}$ & 2.7 \\
& $0.35 \times 10^{-8}$ & $0.36 \times 10^{-6}$ & 0.1 & $0.10 \times 10^{-5}$ & 0.1 \\
\hline 10.0 & $0.86 \times 10^{-10}$ & $0.39 \times 10^{-6}$ & 44.0 & $0.50 \times 10^{-6}$ & 54.7 \\
& $0.86 \times 10^{-9}$ & $0.39 \times 10^{-7}$ & 5.2 & $0.50 \times 10^{-7}$ & 6.1 \\
& $0.86 \times 10^{-8}$ & $0.39 \times 10^{-8}$ & 0.1 & $0.50 \times 10^{-8}$ & 0.2 \\
\hline 15.0 & $0.23 \times 10^{-9}$ & $0.19 \times 10^{-7}$ & 27.9 & $0.21 \times 10^{-7}$ & 36.4 \\
& $0.54 \times 10^{-9}$ & $0.80 \times 10^{-8}$ & 11.3 & $0.90 \times 10^{-8}$ & 14.5 \\
& $0.11 \times 10^{-8}$ & $0.40 \times 10^{-8}$ & 5.3 & $0.45 \times 10^{-8}$ & 6.7 \\
& $0.23 \times 10^{-8}$ & $0.19 \times 10^{-8}$ & 2.2 & $0.21 \times 10^{-8}$ & 2.6 \\
& $0.23 \times 10^{-7}$ & $0.19 \times 10^{-9}$ & 0.05 & $0.21 \times 10^{-9}$ & 0.1 \\
\hline 20.0 & $0.64 \times 10^{-9}$ & $0.19 \times 10^{-7}$ & 12.6 & $0.21 \times 10^{-7}$ & 14.0 \\
& $0.13 \times 10^{-8}$ & $0.80 \times 10^{-8}$ & 6.2 & $0.90 \times 10^{-8}$ & 7.0 \\
& $0.25 \times 10^{-8}$ & $0.40 \times 10^{-8}$ & 2.4 & $0.45 \times 10^{-8}$ & 2.7 \\
& $0.25 \times 10^{-7}$ & $0.19 \times 10^{-8}$ & 0.05 & $0.21 \times 10^{-8}$ & 0.05 \\
\hline \hline
\end{tabular}

\subsubsection{Heavy Charged Lepton $L^{-}$}

We consider the possibility of a heavy charged lepton that decays into a neutrino of its own generation via $L^{-} \rightarrow \nu_{4} W^{*}$ with the pattern of masses $m_{\nu_{4}}<m_{L}<M_{Z} / 2$. It is also assumed that the $\nu_{4}$ is stable to avoid the complication of additional mixing parameters for both the charged lepton and neutrino sectors. The small production rate of $L^{+} L^{-}$events at the $Z$ prohibits direct searches for this process; however, both new particles would now contribute to the visible and total width:

$$
\begin{aligned}
\Gamma_{v i s} & =\Gamma_{v i s}^{0}+\eta_{v i s}\left(\Gamma_{L}+\Gamma_{\nu_{4}}\right) \\
\Gamma_{Z} & =\Gamma_{Z}^{0}+\Gamma_{L}+\Gamma_{\nu_{4}},
\end{aligned}
$$


where $\Gamma_{L}$ is the partial width of the $Z$ to decay into $L^{+} L^{-}$pairs, and $\eta_{v i s}$ is now the efficiency for $L^{+} \rightarrow \nu_{4} W^{*}, L^{-} \rightarrow \nu_{4} W^{*}$ events to satisfy the visible event criteria. In analogy to the unstable $\nu_{4}$ case, the relation:

$$
\frac{\Gamma_{\text {vis }}}{\Gamma_{Z}^{2}}=\frac{\Gamma_{\mathrm{vis}}^{0}+\eta_{\mathrm{vis}}\left(\Gamma_{L}+\Gamma_{\nu_{4}}\right)}{\left(\Gamma_{Z}^{0}+\Gamma_{L}+\Gamma_{\nu_{4}}\right)^{2}}<\frac{\Gamma_{\mathrm{vis}}^{0}}{\left(\Gamma_{Z}^{0}+\left(n_{35 \%}-3\right) \Gamma_{\nu}\right)^{2}}
$$

delimits an exclusion region at $95 \%$ CL. Comparing the above with the expression for an unstable $\nu_{4}$ we see that the addition of $\Gamma_{L}$ allows better limits to be resolved as $\Gamma_{L}$ contributes quadratically to the denominator.

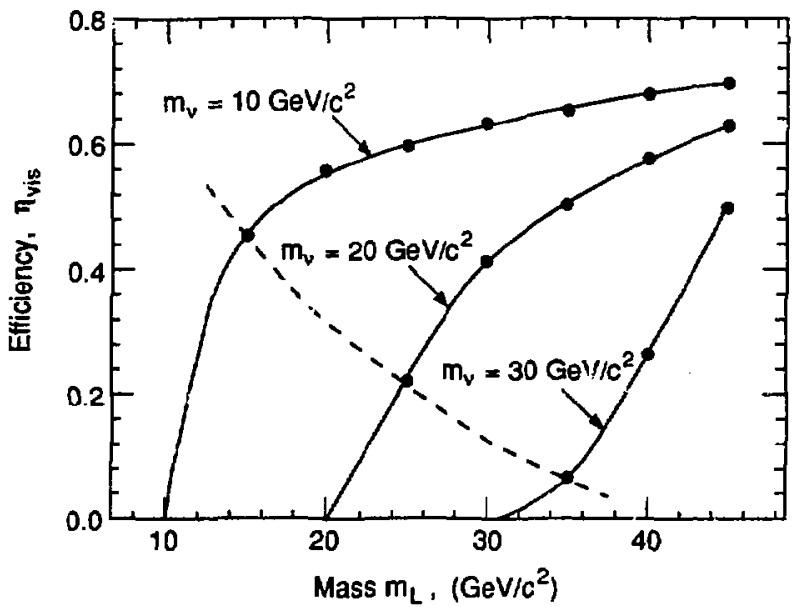

Figure 56: Measured efficiencies $\eta_{v i s}$ to satisfy the visible event criteria for different values of $m_{L}$ and $m_{\nu_{4}}$. MC statistical errors are given by $N_{\text {tot }}^{M C}=3000$ The curves are polynomial interpolations through the measured efficiencies. The dashed line is an example of a curve of constant mass difference $\delta=m_{L}-m_{\nu_{4}}$.

\section{Efficiencies}

The relation of Eq. 85 depends upon the magnitude of both $\Gamma_{L}$ and $\Gamma_{\nu_{1}}$, which in turn are functions of $m_{L}$ and $m_{\nu_{4}}$ rspectively. MC events are generated for various $\left(m_{L}, m_{\nu_{1}}\right)$ pairs, maintaining $m_{v_{1}}<m_{L}$. The efficiency $\eta_{v i s}$ for each pair is found, and the results displayed in Fig. 56. 
From Fig. 56, we see that efficiencies are also strongly dependent upon the mass difference $\delta=m_{L}-m_{\nu_{4}}$. The maximum visible energy in the event is controlled by $\delta$; the smaller the $\delta$, the smaller the maximum visible energy. Efficiencies drop for small $\delta$ because of the visible energy cut V7 and multiplicity cut V8 which are necessary to reduce the backgrounds due to two-photon processes, beam-gas interactions, and beam-beampipe interactions. 


\section{Chapter 6}

\section{Results}

This chapter compares the number of expected new particle and background events after selection cuts with the number observed in the data. Using this information, null direct search results in the form of mass limits and exclusion regions are obtained. The results of fits to the $Z$ resonance data and the upper limit on the invisible width of the $Z$ are presented. This knowledge allows the determination of limits on particle production that contributes to the invisible width.

In Chapter 2, the motivation for searching for the scenarios considered including mass limits at the time of analy $\mathrm{sis}$ were presented. Following the publication of the results of this analysis, the LEP experiments and collaborations were able to incrementally improve on the results of this thesis with their much larger sample of $Z$ decays. This chapter compares our conclusions with these newer LEP limits. Implications for the future are discussed.

\subsection{Expected Number of Events}

\subsubsection{Number of Produced Events}

The expected number of new particle events is normalized to the number of data events $N_{\text {had }}$ which have the characteristics of hadronic $Z$ decays. A typical event in the data displaying a $Z$ decaying into hadrons is shown in Fig. 57. More precisely, $N_{\text {had }}=455$ is the number of data events which fulfill the hadronic visible event selection criteria V1-V8a discussed in the previous chapter. These criteria were also used to choose the events used in the fitting of the $Z$ resonance to the data [66] where hadronic events and $\tau$-pair events with $\left|\cos \theta_{\mathrm{ll} r}\right|<0.65$ needed to be distinguished and be counted separately. This was done 


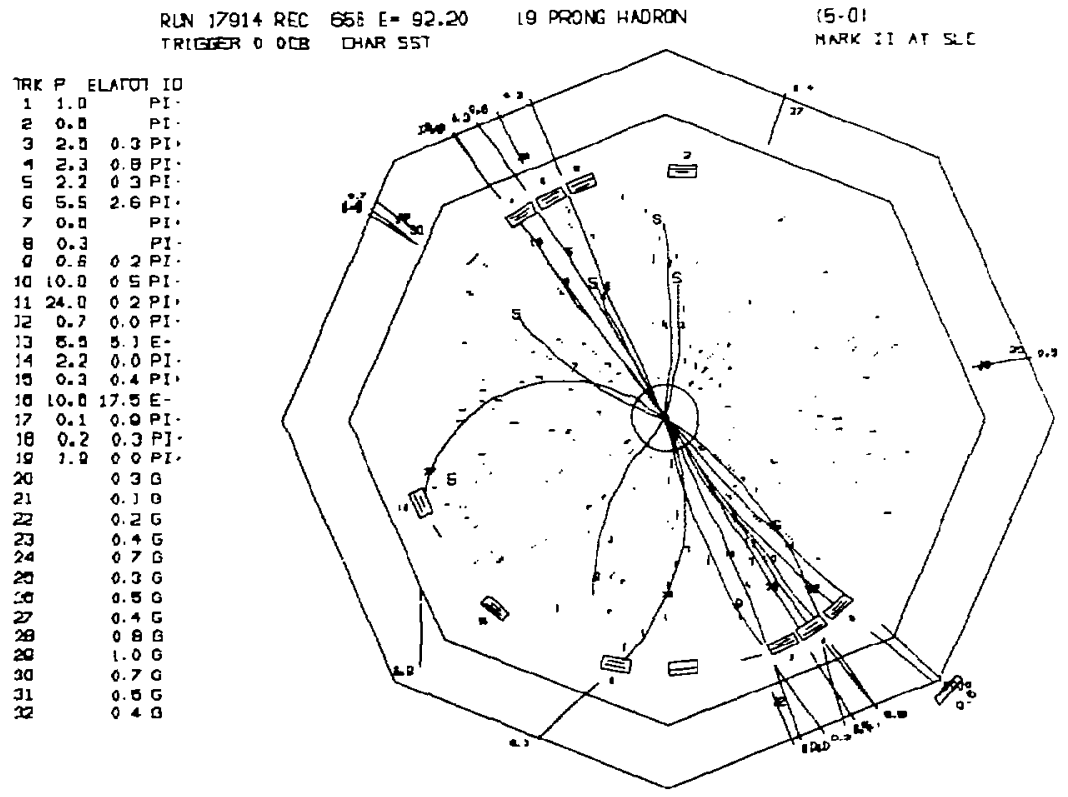

Figure 57: A typical hadronic $Z$ decay in the data.

by a handscan of the candidate data events. However, the efficiency for new particle events to pass the hadronic event cuts needs to be found, and handscanning thousands of MC events is impossible. An event is therefore rejected as a $\tau$-pair event if criteria V1-V7, $2<N_{\text {ch }} \leq 6$, and $T>0.992$ are satisfed. From application of these cuts to MC $\tau$-pair events [121], the efficiency for $\tau$ 's pass the hadronic event cuts is only $(3.4 \pm 0.4) \%$, while the efficiency for udscb events drops negligibly.

Many of the new particle prospects have decay modes which lead to events which will pass these cuts with good efficiency. If $\int \mathcal{L} d t$ denotes the integrated luminosity over time, then

$$
N_{\text {had }}=\int \mathcal{C} d t \cdot\left(\epsilon_{7} \sigma_{q}+\epsilon_{x} \sigma_{s}\right),
$$

where $\epsilon_{\eta}=0.951 \pm 0.007$ is the fraction of $\mathrm{MC} u d s c b$ events that pass the hadronic event cuts, $\sigma_{9}$ is the hadronic $u d s c b \quad Z$ cross section, $\epsilon_{x}$ is the fraction of new particle events that pass the hadronic event criteria, and $\sigma_{x}$ is the cross section for $Z$ new particle events. 
Rearranging, we then have the number of produced new particle events in our data sampic: given by:

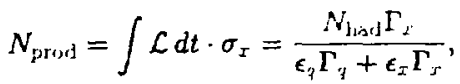

where $\Gamma_{i}$ is the $u d s c b$ hadronic width of the $Z$, and $\Gamma_{s}\left(m_{r}\right)$ is the partial width for the new particle scenario being considered.

In the above calculation, the rotios of partial widths are calculated, and many systematic errors cancel. If the integrated luminosity measured by the SAM and Mini-SAM were used directly, systematic errors from luminosity detector effects and from uncertainties in radiative corrections to the Bhabha cross section would have to be included. The final measurement value for the total integrated luminosity from the SAM and Mini-SAM is

$$
\int \mathcal{L} d t=19.7 \pm 0.8 \mathrm{nb}^{-1}
$$

The error on this number is comparable to the $4.7 \%$ statistical error on $N_{\text {had }}$. The $\int \mathcal{L} d t$ measured for each scanpoint by the luminosity devices predicts $N_{\text {hat }}=464 \pm 19$ events, assuming that $N_{\text {had }}$ contains only udscb quark events. This is consistent with the $N_{\text {had }}=$ 455 events observed, giving another indication of the lack of new particle production. A further advantage of using Eq. 87 is that the effect of the varying $Z$ cross section at the scanpoints corresponding to the ten different $E_{\mathrm{cm}}$ is automatically taken into account.

The calculation of $\Gamma_{y}$ and $\Gamma_{x}$ has been discussed in Chapter 2. The factor $\beta=(1-$ $\left.4 m_{x}^{2} / E_{\mathrm{rm}}^{2}\right)^{1 / 2}$ is found for each event contributing to $N_{\text {had }}$, where $E_{\mathrm{cm}}$ is the value indicated by the energy spectrometer for that event. The partial width $\Gamma_{x}$ is subsequently calculated individually for each event.

The efficiencies $\epsilon_{x}$ are in general weak functions of the new particle mass. For example, $\epsilon_{r}$ varies smoothly from $(97.5 \pm 0.3) \%$ for a $15 \mathrm{GeV} / \mathrm{c}^{2} t$-quark to $(98.4 \pm 0.3) \%$ for a 45 $\mathrm{GeV} / c^{2}$ t-quark (CC decay). Other typical values for $\epsilon_{x}$ are listed in Table 15 . Using MC events, the efficiencies $c_{x}$ are found only at a number of discrete values of $m_{\text {: }}$. Polynomial interpolation [122] is used to estimate values of $\epsilon_{s}$ between these discrete mass values.

The number of produced events expected in the data sample is shown in Fig. 58.

\subsubsection{Errors on the Number of Produced Events}

Lncertainties in the number of produced events $N_{\text {frrid }}$ arise from the $4.7 \%$ statistical error on $N_{\text {lad }}$ and from the small errors on $\epsilon_{i l}$ and $\epsilon_{J}$. The leakage of $\tau$-pair events into the hadronic event sample causes an increase of $<0.14 \%$ in $N_{\text {dersec }}$ and is ignored. Systematic 
Table 15: Efficiencies $\varepsilon_{x}$ at $m_{x}=35 \mathrm{GeV} / c^{2}$ for new particle events to satisfy the hadronic event criteria. These efficiencies should not be confused the detection efficiencies $\epsilon_{D}$ discussed in the previous chapter.

\begin{tabular}{lc|c}
\hline \multicolumn{2}{|c|}{ Process } & $\begin{array}{c}\text { Efficiency } \\
\epsilon_{x}(\%)\end{array}$ \\
\hline \hline$t \rightarrow b W^{*}$ & & $97.9 \pm 0.3$ \\
$t \rightarrow b H^{+}$ & $m_{H}=25 \mathrm{GeV} / c^{2}$ & $95.8 \pm 0.5$ \\
$b^{\prime} \rightarrow b W^{*}$ & & $94.8 \pm 0.5$ \\
$b^{\prime} \rightarrow c H^{-}$ & $m_{H}=25 \mathrm{GeV} / c^{2}$ & $94.3 \pm 0.6$ \\
$b^{\prime} \rightarrow b \gamma$ & & $97.3 \pm 0.3$ \\
$b^{\prime} \rightarrow b g$ & & $96.6 \pm 0.6$ \\
$\nu_{4} \rightarrow e W^{*}$ & $\left|U_{c 4}\right|^{2}=1$ & $94.2 \pm 0.5$ \\
$\nu_{4} \rightarrow \mu W^{*}$ & $\left|U_{\mu 4}\right|^{2}=1$ & $92.1 \pm 0.5$ \\
$\nu_{4} \rightarrow \tau W^{*}$ & $\left|U_{\tau 4}\right|^{2}=1$ & $91.6 \pm 0.6$ \\
\hline \hline
\end{tabular}

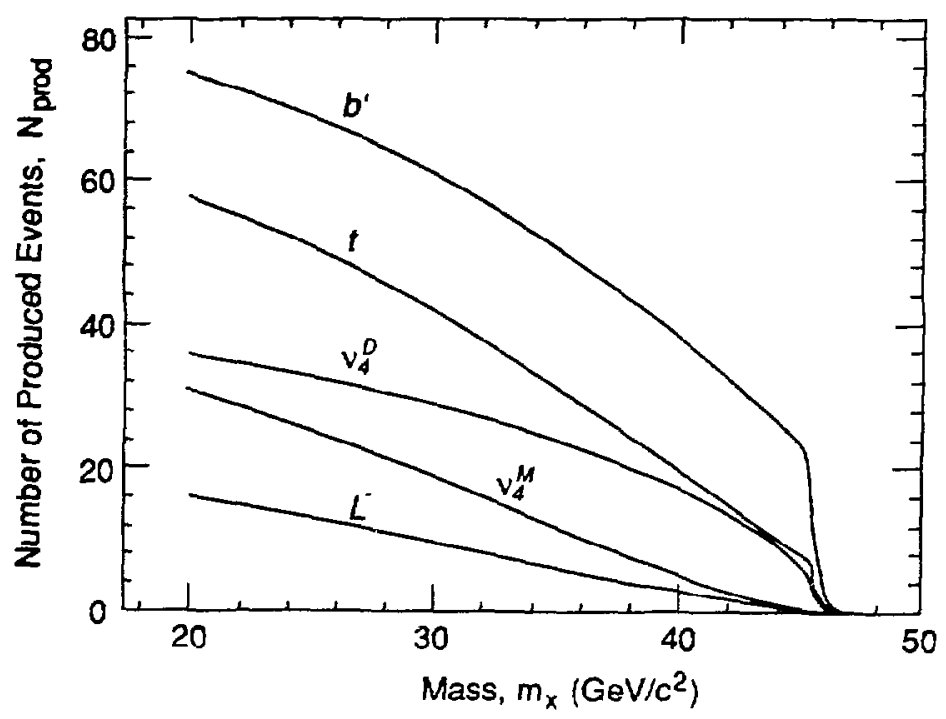

Figure 58: Expected number of produced new particle events in the data set corresponding to $N_{\mathrm{had}}=455$. 
errors on $N_{\text {prad }}$ exist due to uncertainties in the calculation of the $\Gamma_{i}$. These are estimated by varying the input parameters within the following ranges: $M_{Z}=91.1 \mathrm{~s} \pm 0.12 \mathrm{GeV} / \mathrm{c}^{2}$, $\Lambda_{\overline{M S}}=290 \pm 170 \mathrm{MeV}, m_{t}$ from 50 to $200 \mathrm{GeV} / c^{2}$ (except when the expected number of $t$-quark events is being determined), and $m_{H}$ from 30 to $1000 \mathrm{GeV} / c^{2}$. The resulting variations in $N_{\text {prod }}$ lead to the estimation of systematic errors of $1.3 \%$ for $t$-quarks, $1.6 \%$ for $b^{\prime}$-quarks, and $0.3 \%$ for $\nu_{4}$ due to uncertainties in $\Gamma_{i}$.

By far the largest uncertainty for $t$ - and $b^{\prime}$-quarks is from an insufficient knowledge of higher order QCD corrections for massive quarks as discussed in Section 2.1.3 and illustrated in Fig. 11. This translates into an uncertainty on $N_{\text {prod }}$ which varies with quark mass. In the case of the $t$-quark, for masses of $30,35,40$, and $45 \mathrm{GeV} / c^{2}$, we obtain $\Delta N_{\text {prod }} / N_{\text {prod }}=5$, 6,9 , and $19 \%$, respectively.

Errors are propagated in the expression for $N_{\text {prod, }}$ with statistical and individual systematic errors on quantities combined in quadrature.

\subsubsection{Expected Number of Events after Cuts}

The expected number of events $N_{\exp }$ after new particle selection cuts is simply:

$$
N_{\exp }\left(m_{x}\right)=\epsilon_{D}\left(m_{x}\right) \cdot N_{\text {prod }}\left(m_{x}\right),
$$

where $\epsilon_{D}$ is the detection efficiency for the new particle process in question. $N_{\text {exp }}\left(m_{x}\right)$ is found for a number of discrete mass values from MC data sets and polynomial interpolation is used to find values of $N_{\exp }$ between these masses. Errors in $\epsilon_{D}$ discussed in Chapter 5 are combined with the errors on $N_{\text {prod }}$ to give a total error $N_{\exp } \pm \delta_{\text {exp }}$.

\subsection{Mass Limits and Exclusion Regions (Direct Searches)}

The number of data events fulfilling the various new particle selection criteria are compared to the number of new particle events expected $N_{\exp }$, taking predicted backgrounds into account. If there is no apparent signal for a new quark or lepton in the data, we wish to exclude the existence of these particles within a mass range or range of parameters. It is possible to determine the confidence level of exclusion in a number of possible ways. For instance, the shapes of distributious and total number of events observed in the data can be compared to that expected from the sum of backgrounds and hypothetical signal. In this analysis, we pursue a simple and conservative procedure of only comparing the total number of events satisfying our selection criteria. 
The determination of mass limits will be described in more detail using the isolated track topology as an example.

\subsubsection{Isolated Track Topology}

Referring back to the distribution of the $\rho$-parameter in Fig. 45, we observe that the data agrees well with the udseb MC prediction. One data event passes the final cut of $\rho>1.8$. To estimate expected udscb backgrounds, selection cuts are applied to the five Monte Carlo/fragmentation combinations described in Chapter 4. These models predict from $1.1 \pm 0.2$ events (LUND parton shower with Peterson fragmentation, statistical error) to $2.1 \pm 0.3$ events (Webber model) to have $\rho>1.8$. Less than 0.01 two photon events and less than 0.02 beam-gas and beam-beampipe events (at 95\% CL) are estimated to satisfy all the isolated track criteria.

We conclude that there is no compelling evidence for the production of new particles that result in high-momentum isolated tracks in hadronic $Z$ decays.

\section{Background Subtraction}

Since the total number of events observed is very low, techniques based on Poisson statistics are necessary. We follow a recommended [123] procedure for the treatment of Poisson processes with background. Let $N_{0}$ be the number of events observed in the data, $\lambda_{B}$ be the number of events expected from background, and $\lambda_{S}$ be the unknown number of signal events. Conservative upper limits for $\lambda_{S}$ at a given confidence level (CL) are obtained from:

$$
C L=1-\frac{e^{-\left(\lambda_{B}+\lambda_{C L}\right)} \sum_{n=0}^{N_{o}} \frac{\left(\lambda_{B}+\lambda_{C L}\right)^{n}}{n !}}{e^{-\lambda_{B}} \sum_{n=0}^{N_{o}} \frac{\left(\lambda_{B}\right)^{n}}{n !}}
$$

where $\lambda_{C L}$ is adjusted until the above relation is true. For a background of $\lambda_{B}, \lambda_{C I}$, has true probability $\geq C L$ of being larger than $\lambda_{S}$, and is the desired upper limit for $\lambda_{s}$.

A more conservative upper limit is obtained if less background is subtracted. Therefore, we take the smallest value of the number of background events predicted by the various $u d s c b \mathrm{MC}$ generators. For $N_{O}=1$ event, and $\lambda_{B}=1.1$ events, we find that $\lambda_{\mathrm{CI}}=4.1$ events at a CL of $95 \%$. Put another way, if we expect 1.1 events from background, there is only a $5 \%$ probabilicy of 4.1 events luctuating down to the single observed event. 


\section{Isolated Track Mass Limits}

The number of expected CC decay $t \rightarrow b W^{*}$ and $b^{t} \rightarrow c W^{*}$ events fulfilling the isolated track criteria is shown in Fig. 59 as a function of mass $m_{x},\left(x=t, b^{\prime}\right)$. The dashed lines are the number of expected events minus the total error, i.e. $N_{\exp }-\delta_{\text {exp }}$. This quantity takes into account possible fluctuations in Monte Carlo and data statistics, and the various theoretical and systematic uncertainties that affect the calculation of $N_{\text {exp }}$. The dotted line is the 95\% CL upper limit for the observed number of events, including background predictions. The intersection of this line with the lines representing $N_{\exp }-\delta_{\exp }$ delimits the mass range excluded by the present measurement at the $95 \%$ CL.

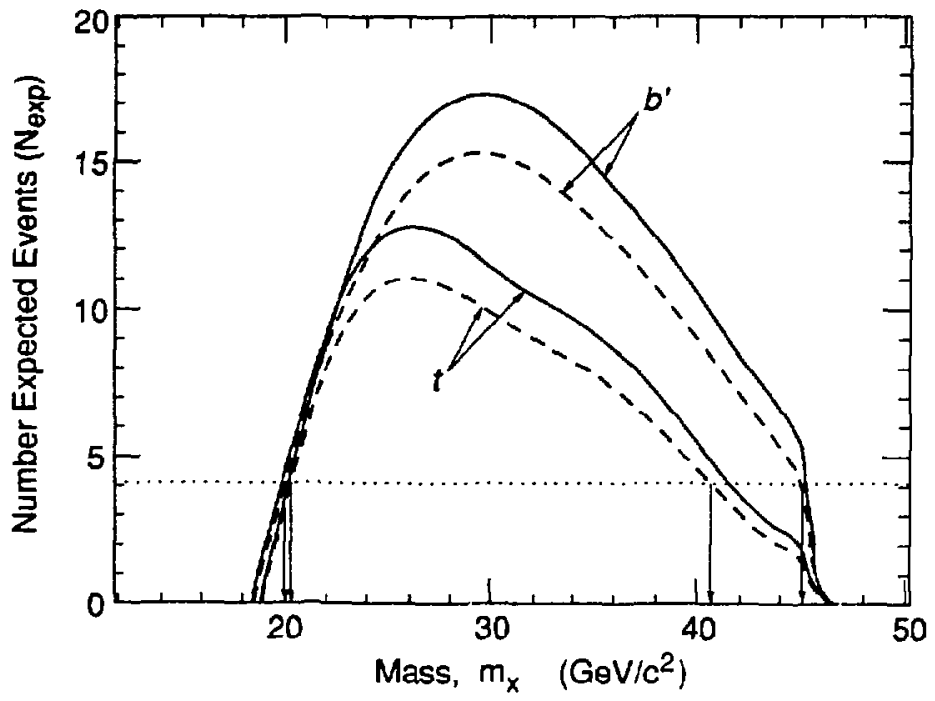

Figure 59: Expected number of $t$ and $b^{\prime}$ (decaying 100\% via CC process) quark events with at least one isolated $\left(\rho_{i}>1.8\right)$ track as a function of the quark mass (solid lines). The dashed curves indicate the central value minus the uncertainty from statistical and systematic errors. The dotted line is the upper bound at $95 \% \mathrm{CL}$ with background subtracted for the observed number of events, and its intersection with the dashed curves delimit the quark mass intervals excluded. 
Therefore, from Fig. 59, the range of masses:

$$
\begin{gathered}
20.1<m_{t}<40.6 \mathrm{GeV} / c^{2} \\
19.9<m_{b^{\prime}}<45.0 \mathrm{GeV} / c^{2},
\end{gathered}
$$

are excluded at $95 \% \mathrm{CL}$ for the case of $100 \% \mathrm{CC}$ decays $t \rightarrow b W^{*}$ and $b^{\prime} \rightarrow c W^{*}$.

Following an identical procedure for short-lived $\nu_{4}$ events, the expected number of events for the possible mixings of $\nu_{4}$ are shown in Fig. 60 for Dirac neutrinos. The variation in $N_{\text {exp }}$ for the different mixing scenarios is due to differences in detection efficiencies explained in Chapter 5. Figure 61 shows the difference between the expected number of events for Dirac and Majorana $\nu_{4}$ for a particular case of mixing to the secord generation. This dissimilarity is dominated by the difference in Dirac and Majorana mass threshold effects and not by differences in detection efficiencies. The $\beta^{3}$ factor always results in fewer $\nu_{4}^{M}$ events than $\nu_{4}^{D}$ events; hence the mass limits for Majorana neutrinos do not cover as large a range of parameter space as Dirac neutrino limits.

Limits on $v_{4}$ production are sensitive to the mixing parameter $U_{\ell 4}$. The isolated track analysis is meant to detect short-lived (prompt) $\nu_{4}$ particles; the detached vertex analysis is sensitive to $\nu_{4}$ particles with longer lifetimes. In order to quantify the requirement of short lifetimes in terms of the mixing matrix element $\left|U_{\ell 4}\right|^{2}$, we conservatively define the detection efficiency for the isolated track topology to be zero if the decay vertex of either $\nu_{4}$ falls outside the cylindrical fiducial region of $\tau<1 \mathrm{~cm},|z|<3 \mathrm{~cm}$ centered at the IP. This allows the track quality cut C1 (requiring charged tracks to project into the above Gducial region) to remain highly efficient. Studies indicate that detection efficiencies for the isolated track topology remain high for much longer decay lengths, but events with these long decay lengths are not pursued since the resultant detached vertices would be observed as signal events by the normalized impact parameter analysis.

Regions excluded by the isolated track analysis in the $m_{\nu_{4}}-\left|U_{\ell_{4}}\right|^{2}$ plane are shown in Fig. 62. Since the $\nu_{4}$ lifetime is inversely proportional to $\left|U_{\ell 4}\right|^{2}$, the described short lifetime requirement leads to a lower bound of sensitivity for $\left|U_{\ell_{4}}\right|^{2}$ seen as diagonal lower edges on the exclusion regions in Fig. 62. These edges are essentially lines of constant $c \tau$, and are slightly different for Dirac and Majorana neutrinos because $\tau\left[\nu_{4}^{D}\right]=2 \tau\left[\nu_{4}^{M}\right]$.

Numerical results for mass limits for prompt $\nu_{4}$ decays are given in Table 16. All results are presented for $100 \%$ mixing to a particular generation. Any arbitrary combination of mixing $|U|^{2}=\left|U_{c 4}\right|^{2}+\left|U_{\mu 4}\right|^{2}+\left|U_{\tau 4}\right|^{2}$ would give mass limits intermediate to the pure mixing cases and bounded by the limits for $100 \% \nu_{4} \rightarrow \mu W^{*}$ and $100 \% \nu_{4} \rightarrow \tau W^{*}$ decays. 


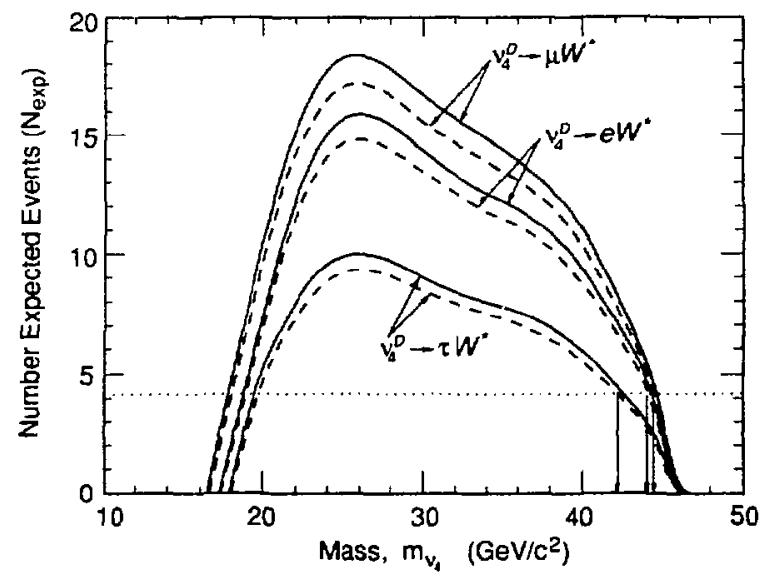

Figure 60: Expected number of $Z \rightarrow \nu_{4}^{D} \bar{\nu}_{4}^{D}$ events (short lived $\nu_{4}$, with $\beta \gamma c \tau<1 \mathrm{~cm})$ with at least one isolated $\left(\rho_{i}>1.8\right)$ track as a function of mass (solid lines). The results for decays via $100 \%$ mixing to each of the three lighter generations are shown. The meanings of the dashed and dotted lines are given in Fig. 59.

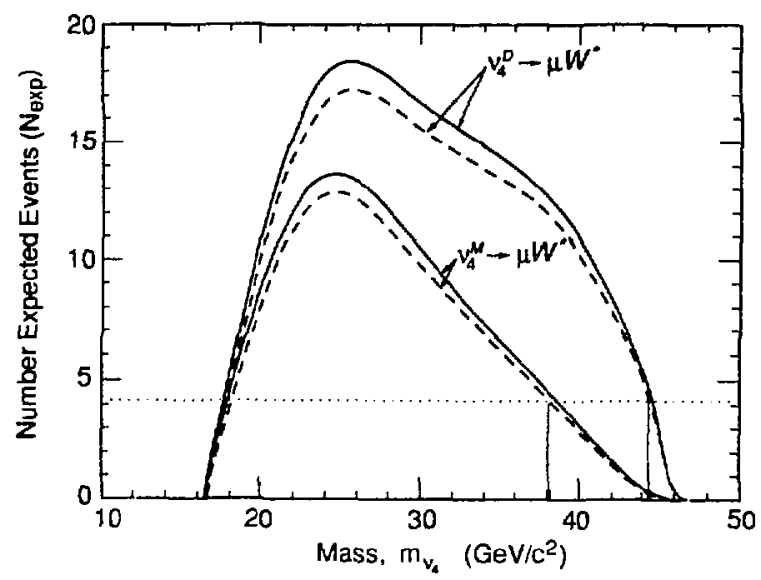

Figure 61: As in Fig. 60, but comparing the number of expected events for Dirac $\nu_{4}^{D}$ and Majorana $\nu_{4}^{M}$ events, both for $100 \%$ mixing to $\nu_{\mu}$. 


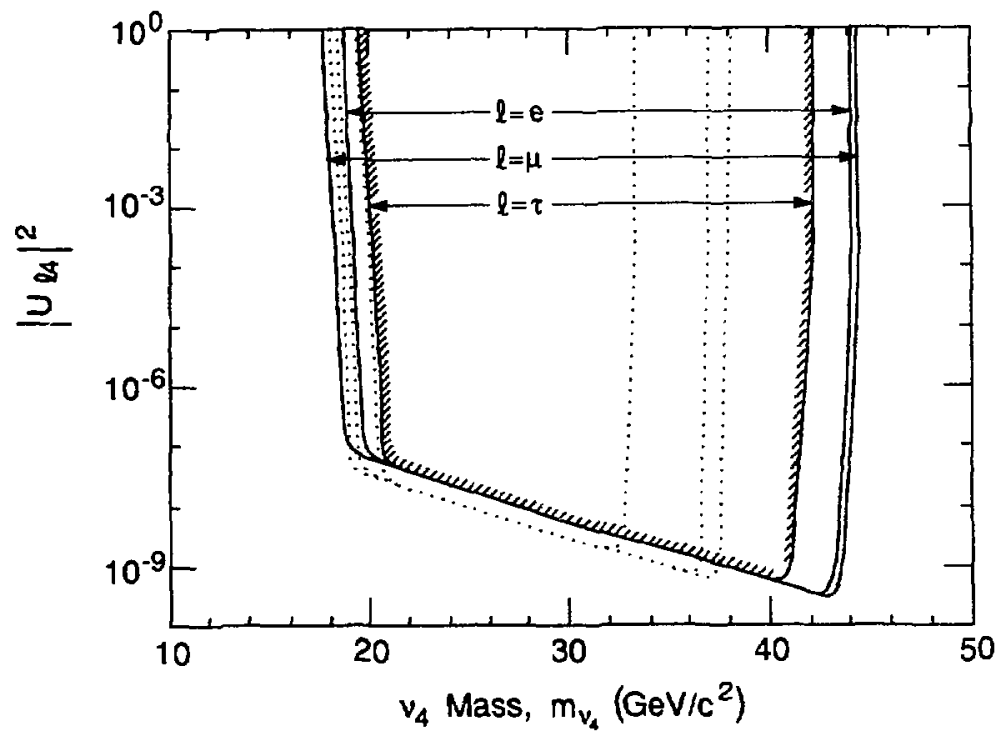

Figure 62: $95 \% \mathrm{CL}$ exclusion regions for Dirac $\nu_{4}^{D}$ production as a function of mass and mixing matrix element $\left|U_{\ell 4}\right|^{2}$ for $\operatorname{Br}\left(\nu_{4} \rightarrow \tau W^{*}\right)=100 \%$ (hatched region, interior to curve), $\operatorname{Br}\left(\nu_{4} \rightarrow \mathrm{eW}\right)=100 \%$, and $\operatorname{Br}\left(\nu_{4} \rightarrow \mu W^{*}\right)=100 \%$ (solid curves as indicated). The corresponding smaller exclusion regions for Majorana $\nu_{4}^{M}$ are shown by the dotted curves.

Table 16: Mass limits from the isolated track analysis for Dirac and Majorana $\nu_{4}$ decaying promptly.

\begin{tabular}{c||c|c}
\hline \multirow{2}{*}{$\begin{array}{c}\text { Mixing Process } \\
(B r=100 \%)\end{array}$} & Mass Range $\left(\mathrm{GeV} / c^{2}\right)$ Excluded at 95\% CL \\
\cline { 2 - 3 } & Dirac, $\nu_{4}^{D}$ & Majorana, $\nu_{4}^{M}$ \\
\hline$\nu_{4} \rightarrow e W^{*}$ & $18.7<m_{\nu}<44.1$ & $18.3<m_{\nu}<37.1$ \\
$\nu_{4} \rightarrow \mu W^{*}$ & $17.7<m_{\nu}<44.4$ & $18.0<m_{\nu}<38.1$ \\
$\nu_{4} \rightarrow \tau W^{*}$ & $19.5<m_{\nu}<42.2$ & $19.4<m_{\nu}<33.4$ \\
\hline
\end{tabular}




\subsubsection{Isolated Photon Topology}

Referring back to Fig. 46, we observe that no data events pass the isolated photon cut of $\rho_{\text {. }}>3.0$. This is consistent with the prediction of $0.36 \pm 0.15 u d s c b$ background events to contain isolated photons. No background subtraction is applied to the zero events observed, and the $95 \%$ CL upper limit for zero events is 3.0 events.

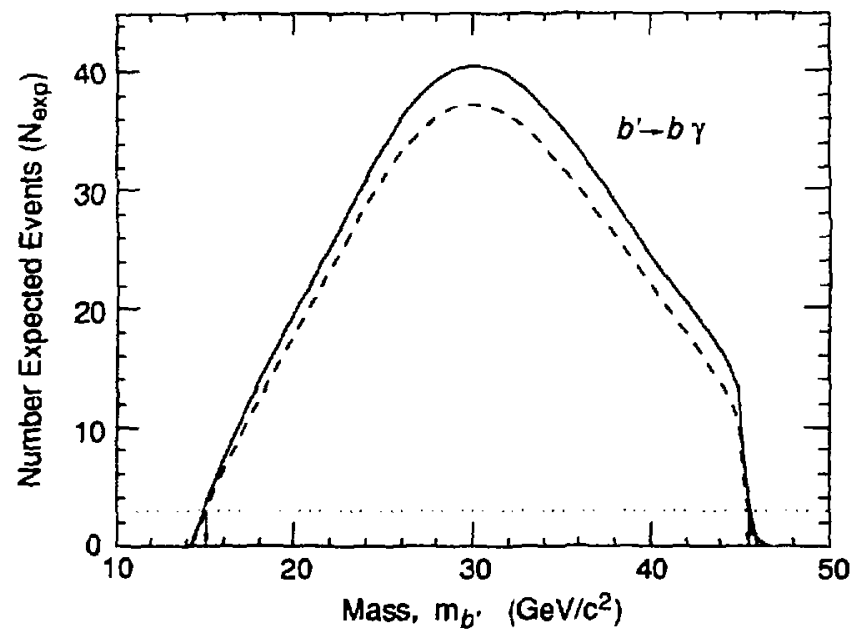

Figure 63: Expected number of $b \rightarrow b y$ events with at least one isolated $\left(\rho_{\gamma_{1}}\right)$ photon as a function of $m_{b^{\prime}}$ (solid curve). The dashed curve indicates the central value minus the uncertainty from statistical and systematic errors. The dotted line is the upper bound at $95 \% \mathrm{CT}$. (3 events) for the zoro observed events.

The expected number of events after selection cuts for the process $Z \rightarrow b^{\prime} b^{\prime}$ with the $b^{\prime}$ decaying $100 \%$ via the FCNC decay $b^{\prime} \rightarrow b \gamma$ is shown in Fig. 63. The intersection of the $N_{\exp }-\delta_{\exp }$ curve with 3.0 events specifies the excluded mass range. We therefore exclude the mass range:

$$
15.1<m_{b^{\prime}}<45.5 \mathrm{GeV} / c^{2}
$$

for a $b^{\prime}$-quark decaying $100 \%$ via $b^{\prime} \rightarrow b y$. Mass limits for other values of the branching fraction for this decay mode will be presented later. 


\subsubsection{Spherical Event Topology}

In Fig. 48, it is observed that the data agrees well with udscb MC predictions. Six events are observed in the data with $M_{\text {out }}>20 \mathrm{GeV} / c^{2}$, while $3.9 \pm 0.5$ events (LUND matrix element model, statistical errors) to $10.6 \pm 0.6$ events (Webber model) are expected from udscb processes. The expected background from two-photon and beam-gas events is negligible. We conclude that there is no convincing evidence for new particle production that results in an excess of spherical events characterized by large momentum sums out of the plane.

The tail of the udscb $M_{\text {out }}$ distribution is very model dependent because of the different ways in which multiple-hard-gluon radiation is handled. It is expected that the LUND matrix element model would predict the fewest number of events with high values of $M_{\text {out }}$, since it uses an exact $\mathcal{O}\left(\alpha_{s}^{2}\right)$ calculation, and can produce a maximum of four partons in the final state (i.e. at most a four jet fnal state, depending on the jet definition). At $E_{\mathrm{cm}} \approx 91 \mathrm{GeV}$, a number of five jet events are measured in the data [124] and are predicted by the other MC models that calculate higher orders through leading-log approximat: These $\geq 5$ jet events contribute significantly to the number of background udscb spherical events. Until higher order calculations are added to the LUND matrix element model, or its parameters tuned using very large samples of hadronic $Z$ lecays, we are ensured that it will give a conservative theoretical prediction of the number of events with large values of $M_{\text {out }}$.

We therefore conservatively perform a background subtraction using the 3.9 events predicted by the LUND matrix element model. Using the procedure outlined earlier we find the upper limit at 95\% CL to be 8.1 events for 6 observed events and 3.9 expected background events. The expected number of $Z \rightarrow b^{\prime} \bar{b}$ events after cuts for various $b^{\prime}$ decay modes is shown in Fig. 64.

From Fig. 64 and an analogous calculation for $t$-quarks, the mass limits of Table 17 are obtained. Note that this analysis is sensitive to the hadronic CC decays of $t$ and $b^{\prime}$, while the isolated track analysis is sensitive to their semileptonic CC decays. The excluded mass ranges for decays through a charged Higgs boson are given for $m_{H}=25 \mathrm{GeV} / \mathrm{c}^{2}$. It is found that for $m_{H} \geq 25 \mathrm{GeV} / c^{2}$, the upper limit of the excluded mass range remains unchanged, and the lower limit is always slightly above the kinematic limit given by $m_{Q}=m_{q}+m_{H}$ for the decay $Q \rightarrow q H$.

The mass limits presented for decays involving $H^{ \pm}$also assume that the charged Higgs 


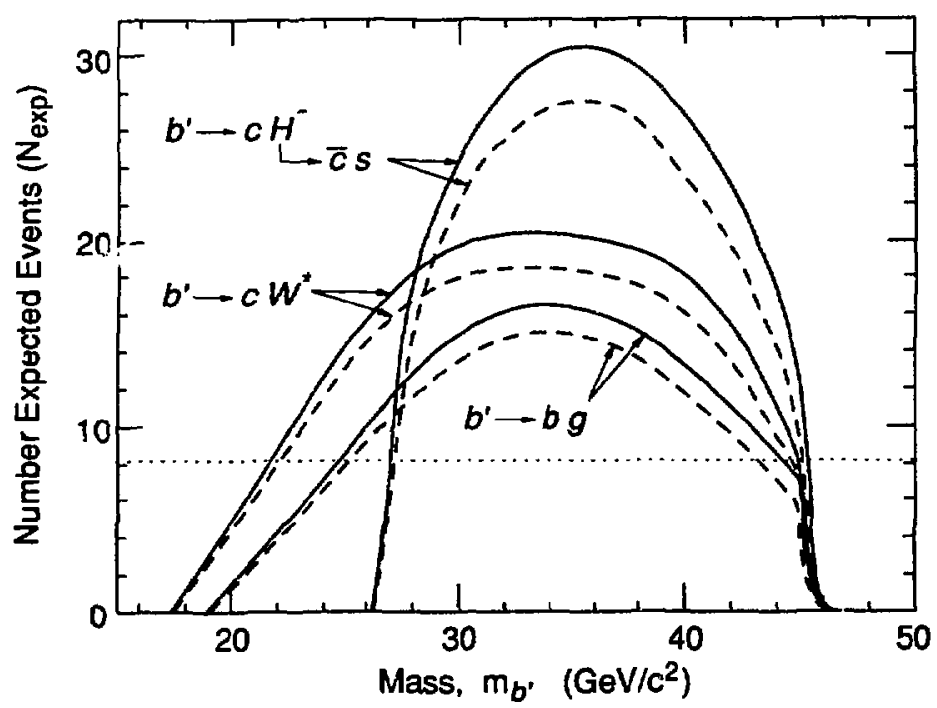

Figure 64: Expected number of $Z \rightarrow b^{\prime} \overline{b^{\prime}}$ events with $M_{\text {out }}>20 \mathrm{GeV} / c^{2}$ for the indicated decay modes $(B r=100 \%)$. The meaning of the dashed and dotted lines are as in Fig. 59.

Table 17: Mass ranges excluded at $95 \%$ CL by the $M_{\text {out }}$ analysis. The upper limits of the excluded ranges for $Q \rightarrow q H$ remain unchanged for $m_{H}>25$ $\mathrm{GeV} / c^{2}$.

\begin{tabular}{l|c}
\hline $\begin{array}{c}\text { Process } \\
(B r=100 \%)\end{array}$ & $\begin{array}{c}\text { Mass Range Excluded at 95\% CL } \\
\left(\mathrm{GeV} / c^{2}\right)\end{array}$ \\
\hline \hline$t \rightarrow b W^{*}$ & $21.0<m_{t}<40.9$ \\
$t \rightarrow b H^{+} \quad m_{H}=25 \mathrm{GeV} / c^{2}, H^{+} \rightarrow c \bar{s}$ & $30.5<m_{t}<42.7$ \\
\hline$b^{\prime} \rightarrow c W^{*}$ & $22.1<m_{b^{\prime}}<44.3$ \\
$b^{\prime} \rightarrow c H^{-} \quad m_{H}=25 \mathrm{GeV} / c^{2}, H^{-} \rightarrow \overline{c s}$ & $27.2<m_{b^{\prime}}<45.0$ \\
$b^{\prime} \rightarrow b g$ & $25.1<m_{b^{\prime}}<43.1$ \\
\hline
\end{tabular}


boson decays $100 \%$ hadronically into $c \bar{s}$ (or $c \bar{b}$, the detection efficiency is found to be identical). These mass limits weaken if the decay $H \rightarrow \tau \nu_{\tau}$ occurs because the low multiplicity of $\tau$ decays and escaping neutrinos give smaller momentum sums out of the event plane than the guarks from hadronic $H^{ \pm}$decays. Using detection efficiencies given by Eq. 69 , mass limits for arbitrary branching ratio $\mathrm{Br}(H \rightarrow c \bar{s})=1-B r\left(H \rightarrow \tau \nu_{\tau}\right)$ are presented in Fig. 65. We conclude that $m_{l}>40 \mathrm{GeV} / c^{2}\left(95 \%\right.$ CL) if $B r\left(H \rightarrow \tau \nu_{\tau}\right)<80 \%$, and that $m_{b^{\prime}}>41.5 \mathrm{GeV} / c^{2}$ regardless of the charged Higgs decay mode.

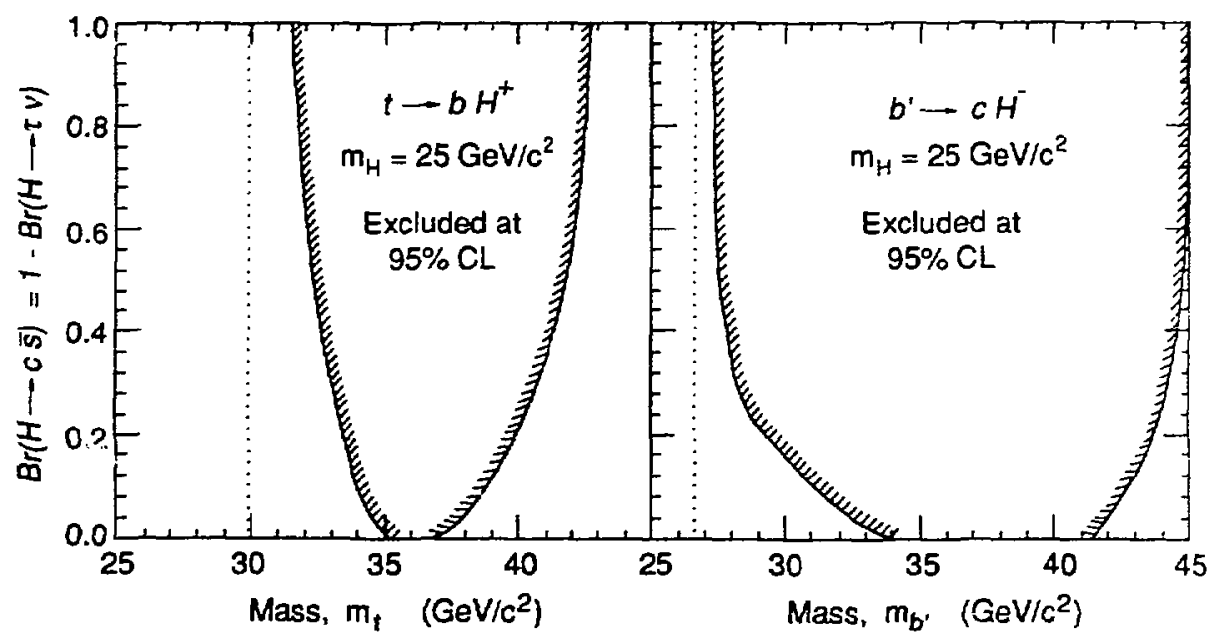

Figure 65: Excluded mass ranges at 95\% CL as a function of the branching ratio $B r(H \rightarrow c \bar{s})=1-B r\left(H \rightarrow \tau \nu_{\tau}\right)$ for $m_{H}=25 \mathrm{GeV} / c^{2}$ for $(a) t \rightarrow b H^{+}$ and (b) $b^{\prime} \rightarrow c H^{-}$. The dotted lines show the kinematic limit for the decays.

\subsubsection{Combined Analysis for $b^{\prime}$}

We now turn our attention to the combination of the isolated photon analysis and $M_{\text {out }}$ analysis described in Section 5.5. All mixtures of the $\mathrm{CC}$ decay mode $b^{\prime} \rightarrow c W^{*}$, and FCNC decay modes $b^{\prime} \rightarrow b g$ and $b^{\prime} \rightarrow b \gamma$ are considered. We assume that decays via a Higgs particle are not kinematically accessible; if they were they would dominate all of the above processes. 


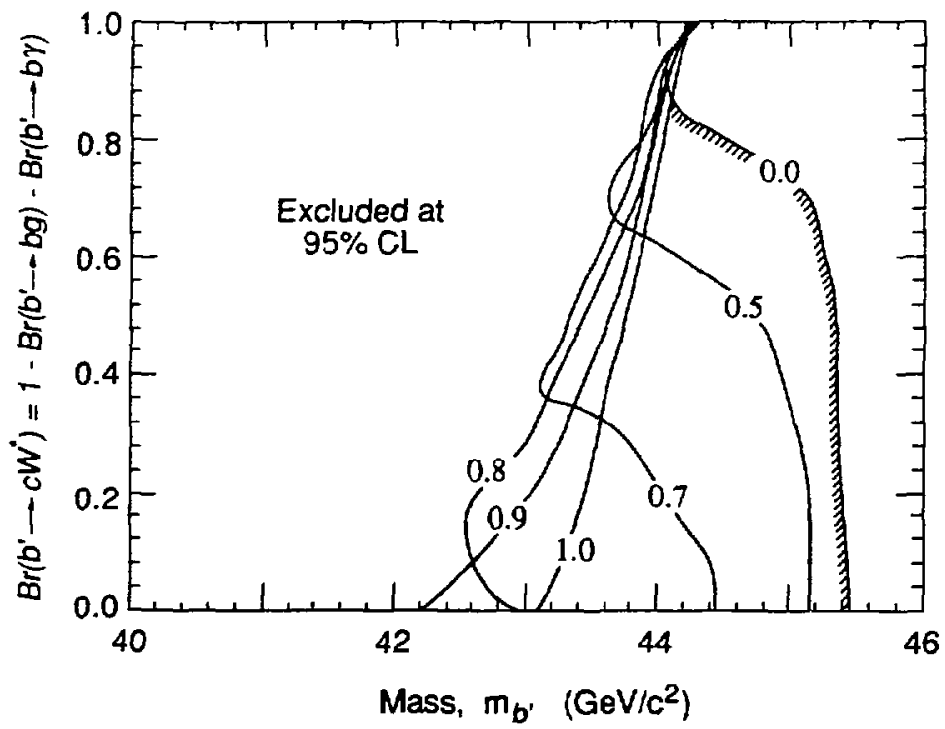

Figure 66: Excluded $b$ mass ranges at 95\% CL as a function of the branching ratio $\operatorname{Br}\left(b^{\prime} \rightarrow c W^{*}\right)$. It is assumed that the remaining decays are the FCNC decays $b^{\prime} \rightarrow b y$ and $b^{\prime} \rightarrow b \gamma$ with the numbers superimposed on the curves being the fraction $\Gamma\left(b^{\prime} \rightarrow b g\right) /\left[\Gamma\left(b^{\prime} \rightarrow b g\right)+\Gamma\left(b^{\prime} \rightarrow b \gamma\right)\right]$. The regions to the left of the curves are excluded for the combinations ghown.

Using the detection efficiency given by Eq. 72, the expected number of events for differing values of $\operatorname{Br}\left(b^{\prime} \rightarrow c W^{*}\right), \operatorname{Br}\left(b^{\prime} \rightarrow b g\right)$, and $\operatorname{Br}\left(b^{\prime} \rightarrow b \gamma\right)$ are found for both the isolated photon and $M_{\text {out }}$ analysis. Mase limits are determined using the appropriate systematic errors and number of observed events for each analysis. The method giving the raore restrictive mass limit is then selected. In this way, the muss limits as a function of $B r\left(b^{\prime} \rightarrow c W^{*}\right)$ shown in Fig. 66 are found for contours of equal value of the fraction $\Gamma\left(b^{\prime} \rightarrow b g\right) /\left[\Gamma\left(b^{\prime} \rightarrow b g\right)+\Gamma\left(b^{\prime} \rightarrow b \gamma\right)\right]$ of the FCNC part.

The distinctive fratures of the $b^{\prime} \rightarrow b \gamma$ decay result in high detection efficiencies and the result that

$$
m_{b^{\prime}}>45.0 \mathrm{GeV} / c^{2} \quad 95 \% \mathrm{CL}\left(\text { for } B r\left(b^{\prime} \rightarrow b \gamma\right)>20 \%\right) \text {. }
$$

It is observed that the least restrictive mass limit results from the combination of branching 
ratios: $\operatorname{Br}\left(b^{\prime} \rightarrow c W^{-}\right)=0, \operatorname{Br}\left(b^{\prime} \rightarrow b g\right)=90 \%$, and $\operatorname{Br}\left(b^{\prime} \rightarrow b \gamma\right)=10 \%$. We conclude that

$$
m_{b^{\prime}}>42.295 \% \mathrm{CL}
$$

regardless of the decay mode of the $b^{\prime}$-quark.

\subsubsection{Detached Vertex Topology}

After all cuts, there are no data events with normalized impact parameter $\chi_{i n p}>0.5$, while MC udscb simulations predict less than 0.2 events. It has also been shown earlier that less than 0.01 beam-gas and beam-beampipe events will survive the selection cuts prior to the $\chi_{\text {imp }}$ cut. The $95 \%$ CL upper limit for the zero observed events is 3.0 events.

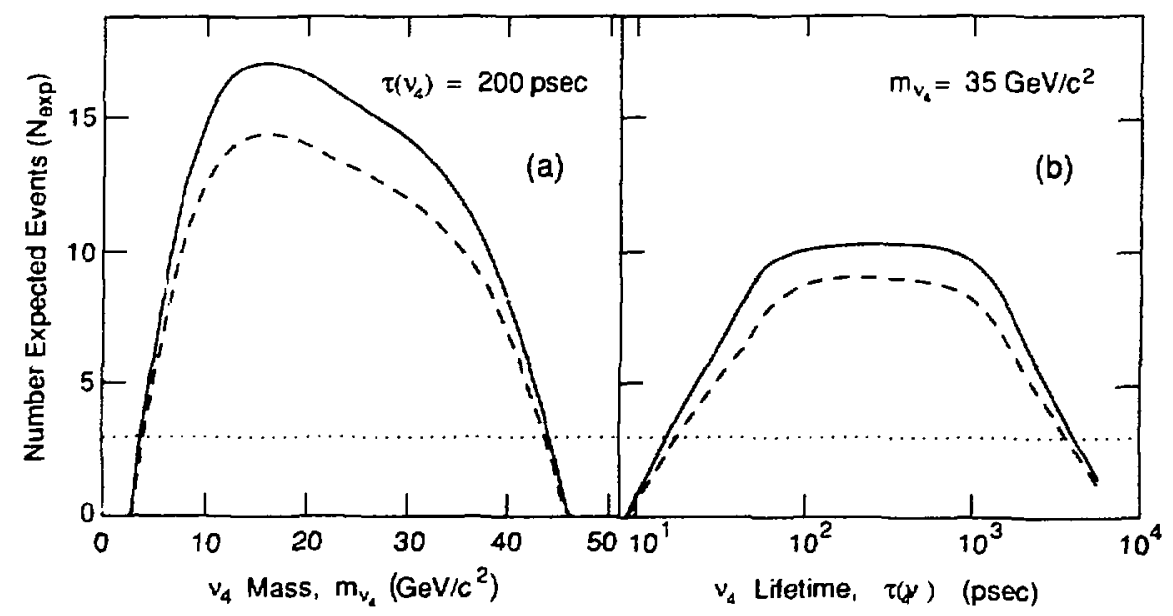

Figure 67: Number of expected long-lived Dirac $\nu_{4} \bar{\nu}_{4}$ events as a function of (a) $\nu_{4}$ mass, and (b) $\nu_{4}$ lifetime. The solid lines are the central values for $N_{\text {exp }}$, and the dashed lines are the central values minus the total estimated error. The dashed line at 3.0 everts represents the $95 \%$ CL upper limit on the zero events observed.

Although the null result of this search can be applied to other neutral or charged longlived particles, we parameterize the results in terms of an hypothesized sequential massive neutrino $\nu_{3}$. The expected number of $\nu_{4} \bar{\nu}_{4}$ events expected $N_{\text {exp }}$ after all cuts is determined for a grid of values in the $m_{\nu 4}-\left|U_{l 4}\right|^{2}$ plane. Two-dimensional polynomial interpolation 
is used to estimate $N_{\exp }$ between grid points. A sample projection of $N_{\exp }$ is shown in Fig. 67(a) as a function of $\nu_{4}$ mass and in Fig. 67(b) as a function of $\nu_{4}$ lifetime.

The number of expected events falls for high mass $\nu_{4}$ because of the decreasing cross section for $\nu_{4} \bar{\nu}_{4}$ production. $N_{\text {exp }}$ also decreases as the $\nu_{4}$ lifetime decreases since the impact parameters will be too small to be distinguished using the normalized impact parameter method. If the $\nu_{4}$ lifetime becomes too long, a significant fraction of neutrinos will decay outside of the detector volume, also causing a drop in $N_{\text {exp }}$.

A conservative $95 \%$ limit contour is found by decreasing $N_{\text {exp }}$ by the total error, and determining the 3.0 event contour. The results are shown in Fig. 68 for Dirac and Majorana neutrinos.

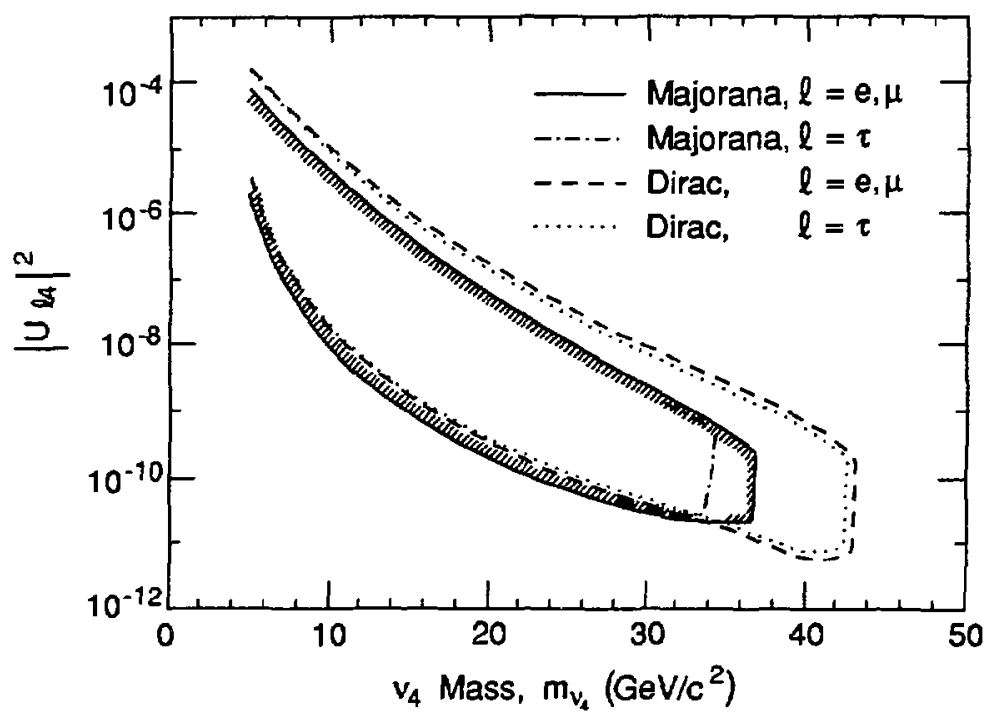

Figure 68: 95\% CL $\nu_{4}$ exclusion regions from the normalized impact parameter analysis as a function of $\nu_{4}$ mass and mixing matrix element.

The upper and lower boundary of the Dirac $\nu_{4}$ limit contour $(\ell=e, \mu)$ at $m_{\nu_{4}}=35$ $\mathrm{GeV} / c^{2}$ correspond to mean decay lengths of $0.6 \mathrm{~cm}$ and $83 \mathrm{~cm}$, respectively. The exclusion regions for $\ell=\tau$ are smaller because of the slightly smaller detection efficiencies for mixing to $\nu_{\tau}$. Again, all results are presented for $100 \%$ mixing to a particular generation. Any 
arbitrary combination of mixing would give exclusion regions intermediate to the pure mixing cases.

\subsection{Limits from Measurements of $Z$ Resonance Parameters}

\subsection{1 $Z$ Resonance Fit Results}

We consider the measurement of the $Z$ to invisible decay modes to further exclude certain regions of the mass and mixing values of fourth generation neutrinos and charged leptons. The details of the resonance fit are found elsewhere [66]; we brielly present the fit procedure and results in order to reinterpret the measurement of the upper limit on the number of massless neutrino generations $N_{\nu}$. The average energy, integrated luminosity, number of events, and measured cross section $\sigma_{Z}$ is given in Table 18.

Table 18: Average energy, integrated luminosity, numher of SAM ( $\left.n_{S}\right)$ and Mini-SAM $\left(n_{M}\right)$ luminosity events [66], number of visible hadronic $\left(N_{\text {had }}\right)$, $\mu$-pair $\left(N_{\mu}\right)$, and $\tau$-pair $\left(N_{\tau}\right) Z$ decays, and measured visible cross section $\sigma_{Z}$ for each energy scan point.

\begin{tabular}{rrrcrrrr}
\hline \hline \begin{tabular}{c}
\hline$E\rangle$ \\
$(\mathrm{GeV})$
\end{tabular} & $n_{S}$ & $n_{M}$ & $\begin{array}{c}c \mathcal{L} d t \\
\left(\mathrm{nb}^{-1}\right)\end{array}$ & \multicolumn{3}{c}{$Z$ Decays } & \multicolumn{1}{c}{$\sigma_{Z}$} \\
\hline \hline 89.24 & 24 & 166 & $0.68 \pm 0.05$ & 3 & 0 & 0 & $4.5_{-2.5}^{+4.5}$ \\
89.98 & 36 & 174 & $0.76 \pm 0.05$ & 8 & 1 & 1 & $13.5_{-4.3}^{+6.0}$ \\
90.35 & 132 & 684 & $3.06 \pm 0.11$ & 65 & 1 & 2 & $23.2_{-3.2}^{+3.7}$ \\
90.74 & 54 & 266 & $1.21 \pm 0.07$ & 33 & 1 & 5 & $31.7_{-5.5}^{+6.8}$ \\
91.06 & 170 & 923 & $4.08 \pm 0.12$ & 114 & 3 & 3 & $31.6_{-3.1}^{+3.4}$ \\
91.43 & 164 & 879 & $4.12 \pm 0.13$ & 108 & 3 & 3 & $29.8_{-2.9}^{+3.3}$ \\
91.50 & 53 & 275 & $1.23 \pm 0.07$ & 33 & 1 & 5 & $34.3_{-5.7}^{+7.0}$ \\
92.16 & 31 & 105 & $0.54 \pm 0.05$ & 11 & 0 & 0 & $21.5_{-6.6}^{+9.2}$ \\
92.22 & 128 & 680 & $3.05 \pm 0.11$ & 67 & 2 & 2 & $24.3_{-3.0}^{+3.4}$ \\
92.96 & 39 & 214 & $1.00 \pm 0.07$ & 13 & 0 & 1 & $14.6_{-4.0}^{+5.4}$ \\
\hline Totals & 815 & 4299 & $19.7 \pm 0.8$ & 455 & 13 & 18 & \\
\hline \hline
\end{tabular}

To perform the resonance fits to the data, a likelihood function is constructed from the 
probability of observing, at each energy, $n_{Z} Z$ decays and $n_{L}$ SAM and Mini-SAM Bhabha luminosity events given that a total of $n_{Z}+n_{L}$ events have been observed. The likelihood $L$ is obtained as:

$$
L=\prod_{\substack{\text { scan } \\ \text { points }}} \frac{\left(\epsilon_{\mathrm{vis}} \sigma_{Z}\right)^{n_{Z}}}{\left(\epsilon_{\mathrm{vis}} \sigma_{Z}+\sigma_{L}\right)^{n_{Z}+n_{L}}}
$$

where $\epsilon_{\mathrm{vis}}=0.954 \pm 0.008$ is the overall efficiency (see Section 5.7.2). Systematic errors from luminosity detector effects, the uncertainty in the absolute energy scale, and the uncertainty in $\epsilon_{\mathrm{vis}}$ are included by the addition of penalty functions to the expression for $L$. As described earlier, $M_{Z}$ and $N_{\nu}$ are left as free parameters, and $\Gamma_{\nu}$ and all other parameters are fixed at their Standard Model values. In this parameterization, $N_{\nu}$ is derived largely from $\sigma_{\text {peak }}$, the height of the resonance.

The negative of the logarithm of the likelihood $-\ln L$ is minimized and the resulting fit and data is shown in Fig. 69. The CL corresponding to $x$ standard deviations is taken to be the point at which $\ln L$ decreases by $x^{2} / 2$ from its maximum value.

The results of the fit are:

$$
\begin{aligned}
M_{Z} & =91.14 \pm 0.12 \mathrm{GeV} / c^{2} \\
N_{\nu} \Gamma_{\nu} & =0.45 \pm 0.10 \mathrm{GeV}, \text { and } \\
N_{\nu} & =2.8 \pm 0.6 \text { Generations }
\end{aligned}
$$

Referring to Fig. 70, the one-sided 95\% CL (1.64 standard devistions) upper limit on $N_{\nu}$ occurs at the point where $-\ln L$ drops by $(1.64)^{2} / 2$ from its maximum value. Therefore,

$$
N_{\nu}<3.86(95 \% \mathrm{CL})
$$

A total systematic error corresponding to 0.45 massless neutrino generations from uncertainties in the absolute cross section and scale factors for luminosity events is included in this result.

\subsubsection{Stable $\nu_{4}$ Mass Limits}

$N_{\nu}$ is a count of massless neutrino generations. A massive neutrino contributes a fractional neutrino generation due to a mass threshold factor, and the upper limit on $N_{\nu}$ can be translated into a mass limit on a stable fourth generation neutrino. Reiterating results of Chapter 2, if we assume that the neutrinos of the first three generations are 


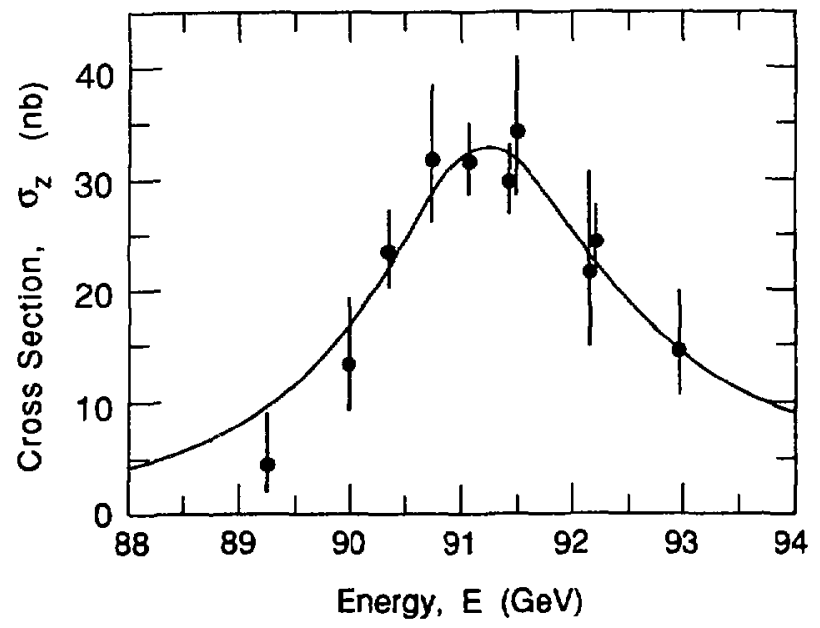

Figure 69: Measured $\sigma_{Z}$ cross sections for $Z$ decays to all hadronic events plus $\mu$ and $\tau$-pair events with $\left|\cos \theta_{\text {thr }}\right|<0.65$ (points with errors) and the fit to the $Z$ resonance (solid line) with $M_{Z}$ and $\Gamma_{\text {inv }}$ as free parameters.

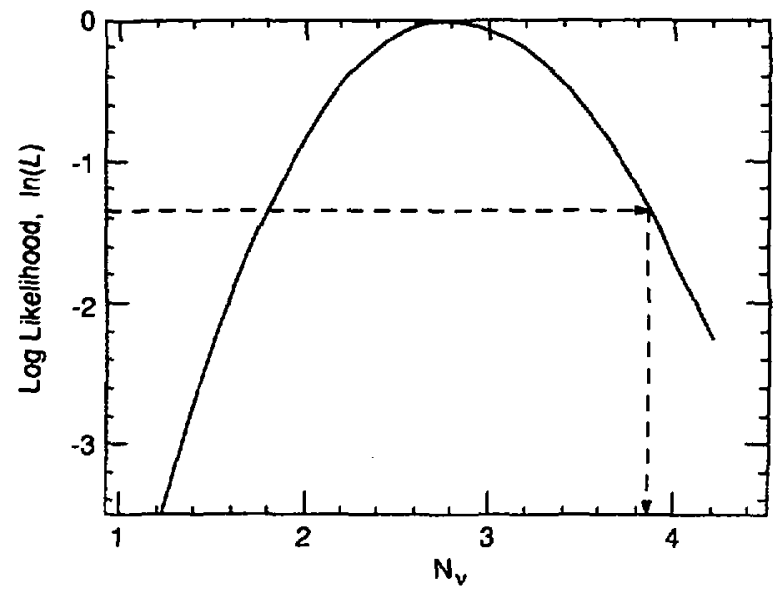

Figure 70: Log likelihood for $Z$ resonance fit as a function of the number of massless neutrino generations $N_{\nu}$ indicating a measurement of $N_{\nu}=2.8 \pm 0.6$. The dashed lines show the one-sided $95 \%$ CL lir: 6 or $N_{\nu}<3.86$. 
massless, and that no other new physics intervenes, then

$$
N_{\nu}=3.0+ \begin{cases}\beta\left(3+\beta^{2}\right) / 4 & \text { Dirac; } \\ \beta^{3} & \text { Majorans, }\end{cases}
$$

where $\beta=\left(1-4 m_{\nu_{4}}^{2} / s\right)^{1 / 2}$ is the velocity of the $\nu_{4}$ in the center-of-mass frame. These functions are plotted in Fig. 71. If $\nu_{4}$ is massless, then $N_{\nu}=4.0$. If $m_{\nu_{4}}>M_{Z} / 2$, then $\nu_{4}$ is not pair-produced in $Z$ decays, and $N_{\nu}=3.0$. We see from Fig. 71 that the limit $N_{\nu}<3.86$ at $95 \%$ CL therefore implies

$$
\begin{array}{ll}
m_{\nu_{4}}>19.6 \mathrm{GeV} / c^{2} & \text { Stable Dirac } \\
m_{\nu_{4}}>14.2 \mathrm{GeV} / c^{2} & \text { Stable Majorana }
\end{array}
$$

at $95 \% \mathrm{CL}$ for a stable $\nu_{4}$ which contributes exclusively to the invisible width of the $Z$.

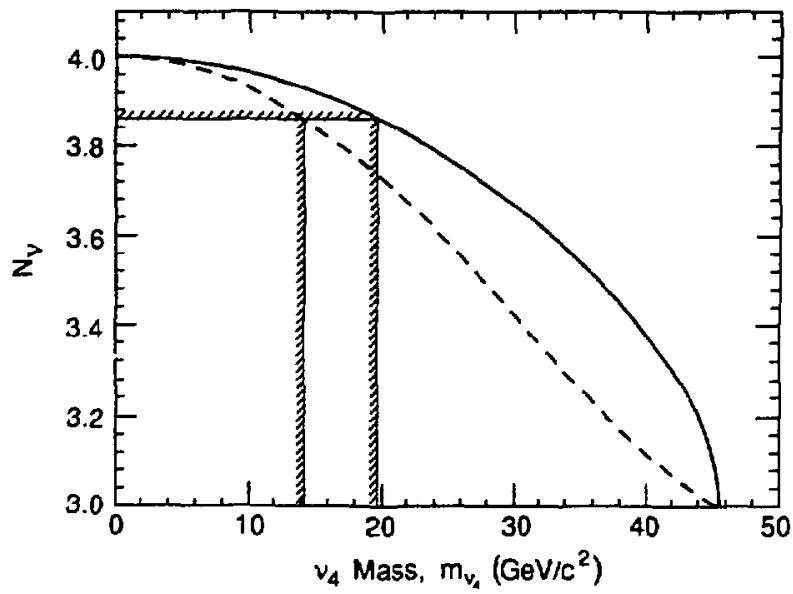

Figure 71: Total number of neutrino generations $N_{\nu}$ as a function of mass $m_{\nu_{4}}$ of a fourth generation stable Dirac (Bolid line) or Majorana (dashed line) neutrino. The limits from $N_{\nu}<3.86$ are shown. 


\subsubsection{Unstable $\nu_{4}$ Limits}

As described in Section 5.7.3, if a hypothetical $\nu_{4}$ is unstable, it will begin to contribute to the visible and total width of the $Z$. We have $n_{95 \%}=3.86$ in Eq. 83 , and the region where:

$$
\frac{\Gamma_{\mathrm{vis}}}{\Gamma_{Z}^{2}}=\frac{\Gamma_{\mathrm{vis}}^{0}+\eta_{\mathrm{vis}} \Gamma_{\nu_{4}}}{\left(\Gamma_{Z}^{0}+\Gamma_{\nu_{4}}\right)^{2}}<\frac{\Gamma_{\mathrm{vis}}^{0}}{\left(\Gamma_{Z}^{0}+(3.86-3) \Gamma_{\nu}\right)^{2}}
$$

is true is excluded at $\geq 95 \%$ CL. Setting the inequality to an equality, we solve for the quantity $\eta_{\text {vis }}\left(95 \%\right.$ CL) shown in Fig. 72 as a function of $m_{\nu_{4}}$ for a Dirac neutrino. It can be interpreted as the maximum efficiency possible for $\nu_{4} \bar{\nu}_{4}$ events to contribute to $\Gamma_{\text {vis }}$ such that $m_{\nu,}$ is the $95 \%$ CL lower mass limit on a fourth generation neutrino. As $\eta_{v i s}(95 \%$ CL) increases from zero (stable neutrino), the lower mass limit will degrade.

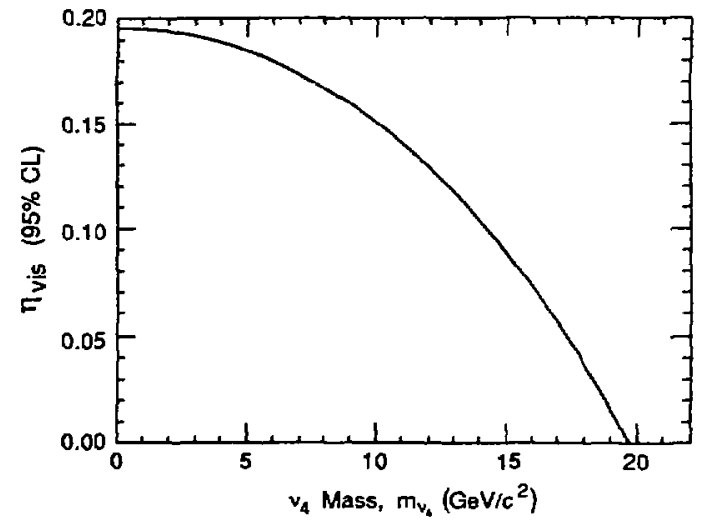

Figure 72: Maximum efficiency possible for $\nu_{4} \bar{\nu}_{4}$ events to contribute to $\Gamma_{\text {vis }}$ such that $m_{\nu_{4}}$ is the $95 \%$ CL lower mass limit for a Dirac $\nu_{4}$.

The efficiencies $\eta_{v i s}$ for $\nu_{4} \bar{\nu}_{4}$ events to pass the visible width cuts have already been measured for different values of $m_{\nu_{4}}$ and $\left|U_{\ell 4}\right|^{2}$. Two-dimensional interpolation is used to estimate $\eta_{v i s}$ between the MC data set values. For a given $m_{v_{4}}$ the value of $\left|U_{t 4}\right|^{2}$ whert $\eta_{\text {vis }}$ satisfies Eq. 101 defines the 95\% exclusion regions shown in Fig. 73.

As a consistency check on this procedure, the fit is redone with the same data sample, but constraining $\Gamma_{v i s}$ to be equal to $\Gamma_{v i s}^{0}+\eta_{v i s} \Gamma_{\nu_{4}}$. The new fit value of $N_{\nu}$ is found and the mass limit on $m_{\nu 4}$ is checked. For example, if $\eta_{v i s}$ is set to 0.195 , then a refit gives 


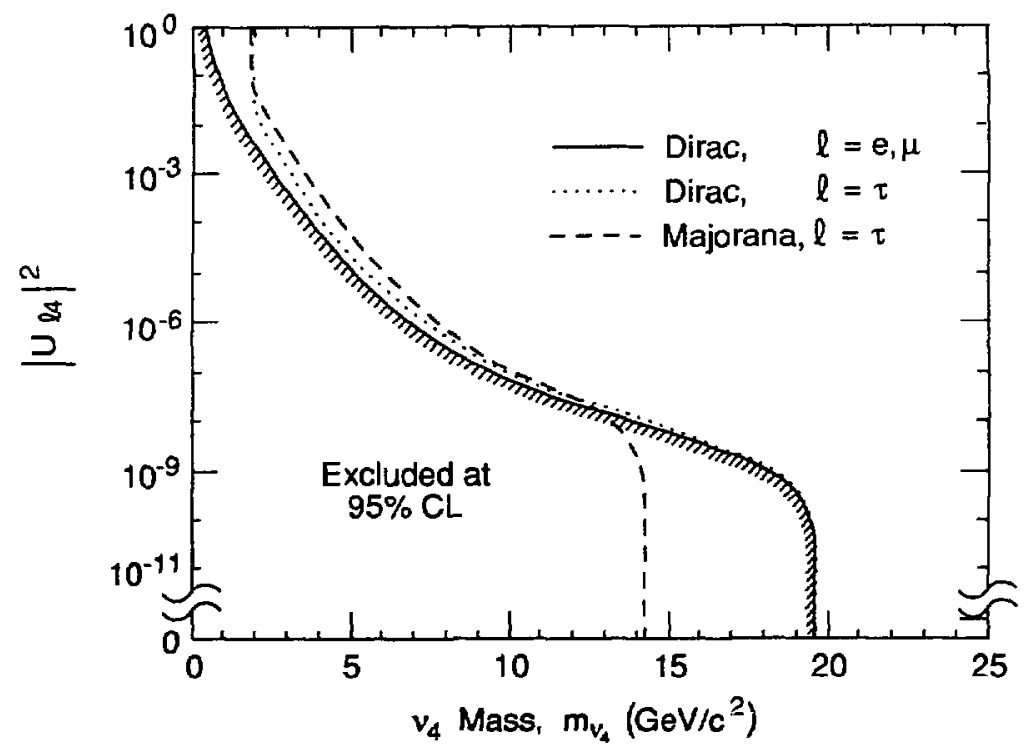

Figure 73: $95 \%$ exclusion regions for $\nu_{4}$ from $N_{\nu}<3.86$ in the mass versus mixing matrix element plane.

$N_{\nu}<4.0$, i.e. the $\nu_{4}$ must be massless, as indicated in Fig. 72 . The data was refit using a number of different input $\Gamma_{v i s}$ values, and in all cases, the resultant mass limit is consistent with the value given by $\eta_{\text {vis }}(95 \% \mathrm{CL})$.

\subsubsection{Heavy Charged Lepton $L^{-}$- Iimits}

An identical procedure is followed to place limits on the mass of a heavy charged lepton $L^{-}$and its stable neutrino $\nu_{L}$ (i.e. $\nu_{4}$ with no mixing), except that now the relation

$$
\frac{\Gamma_{\text {vis }}}{\Gamma_{Z}^{2}}=\frac{\Gamma_{v i s}^{0}+\eta_{\text {vis }}\left(\Gamma_{L}+\Gamma_{\nu_{4}}\right)}{\left(\Gamma_{Z}^{0}+\Gamma_{L}+\Gamma_{\nu_{i}}\right)^{2}}<\frac{\Gamma_{\text {vis }}^{0}}{\left(\Gamma_{Z}^{0}+(3.86-3) \Gamma_{\nu}\right)^{2}}
$$

needs to be satisfied. $\eta_{\text {vis }}$ is now the efficiency for $Z \rightarrow L^{+} L^{-}$events to satisfy the visible event criteria. The $95 \%$ CL exclusion region of Fig. 74 is obtained. 


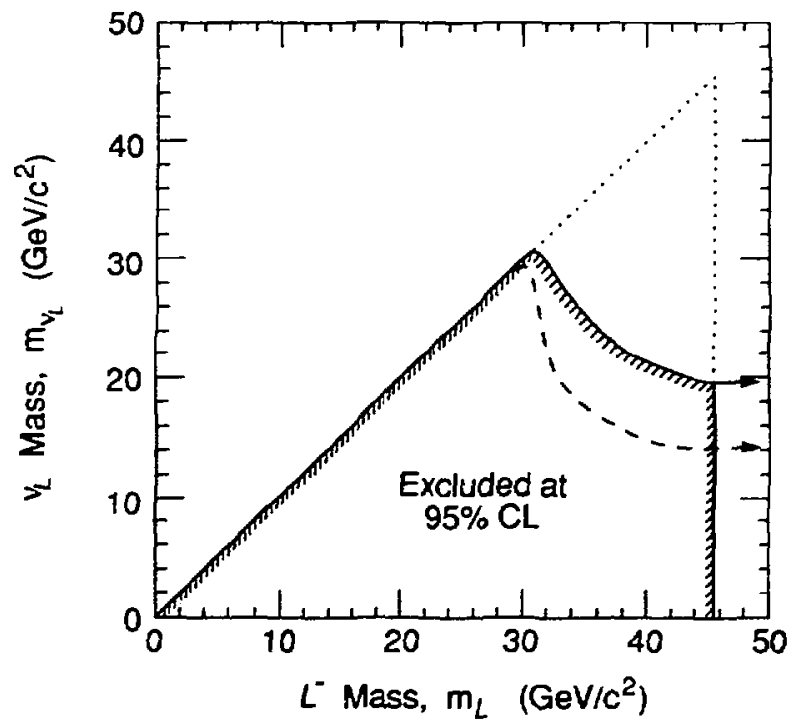

Figure 74: 95\% exclusion regions in the charged lepton mass $m_{L}$ versus otable neutrino mase $m_{\nu_{L}}$ plane for a Dirac $\nu_{L}$ (solid line) and a Majorana $\nu_{L}$ (dashed line).

\subsection{Summary and Comparisons}

\subsubsection{Top Quark}

The excluded mases ranges for the t-quark of this work extend the previous TRISTAN mass limits [41] shown graphically in Fig. 75. Our limits overlap with the lower range of top mase sensitivity of the $p \bar{p}$ collider $C C$ decay searches $[42,43,44]$ that give a lower limit on $m_{t}$ of around $80 \mathrm{GeV}$. Shortly after the publication of the Mark II mass limits, the LEXP experiments, with a larger data sample of $Z$ decays, obtained [125] slightly more restrictive mass exclusion ranges also shown in Fig. 75 . The LEP results are consistent with the Mark II results.

Including consideration of the decay $t \rightarrow b H^{+}$provides a model independent and inambiguous linit on the t-quark mass. These mass limits weaken for large values of $\operatorname{Br}\left(H \rightarrow \tau \nu_{\tau}\right)$, but a combination [126] of our result with the $B-\widetilde{B}$ mixing measurement of the ARGUS 


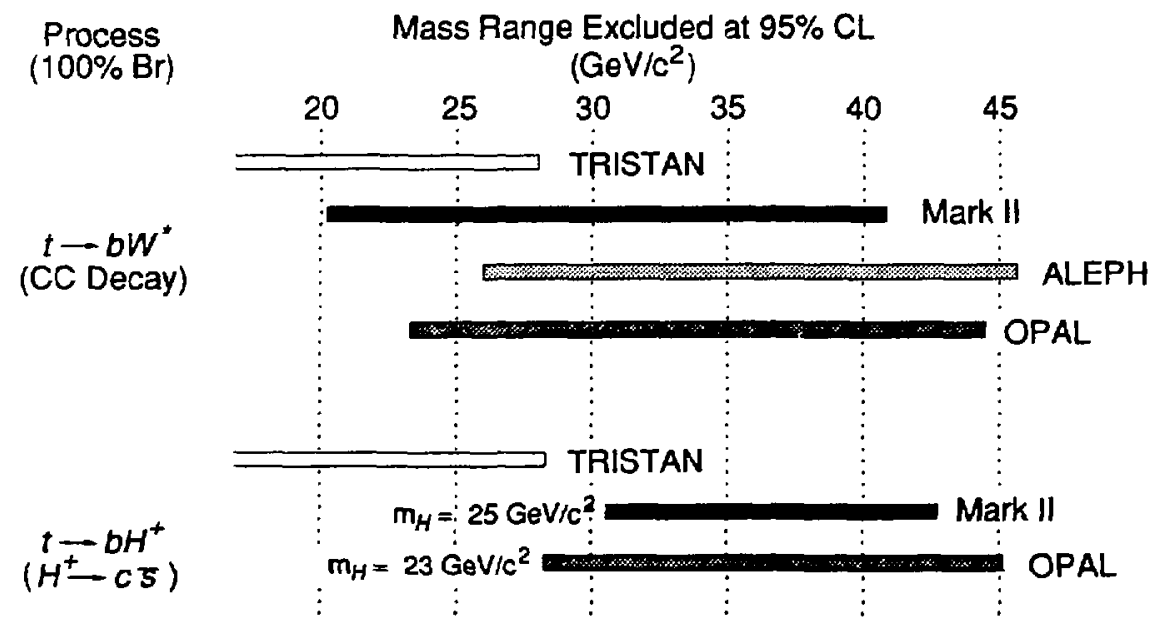

Figure 75: Summary and comparison of t-quark mass limits from $e^{+} e^{-}$colliders. The TRISTAN results existed at the time of the analysis, while the OPAL and ALEPH limits were published [125] shortly after the publication of the limits of this thesis.

and CLEO collahorations [4] gives a mass limit of $m_{t}>40 \mathrm{GeV} / c^{2}$ regardless of the decay mode of the charged Higgs boson. Lirnits of $m_{H^{ \pm}}>35$ to $43 \mathrm{GeV} / c^{2}$ (depending on the $H^{ \pm}$decay modes) have also been recently set by LEP experiments [128], thereby almost eliminating the possibility of decays through $H^{ \pm}$for $m_{t}<M_{Z} / 2$. Decays through $H^{ \pm}$are difficult to detect at a $p \bar{p}$ collider, but the UAl collaboration [127] has recently outlined how they would search for the decay $t \rightarrow b H^{+}$in a larger data sample, and present limits for decays through a virtual $H^{ \pm}$(i.e. $m_{t}<m_{H}+m_{b}$ ).

Recently available high statistics data on the $Z$ mass and partial decay widths can be used together with the world average of $\sin ^{2} \theta_{W}$ to estimate $m_{l}$ through its effect on radiative corrections to various measured quantities. The best fitted value [129] is $m_{t}=137_{-42}^{+37}$ $\mathrm{GeV} / c^{2}$ with $90 \% \mathrm{CL}$ upper and lower limits on $m_{t}$ of 195 anc $65 \mathrm{GeV}$ respectively. Due to the vertex correction to the $Z$ to $b \bar{b}$ partial width, which is specifically dependent on the top quark mass, it is also possible to disentangle the effect of the top quark mass from other radiative corrections to $\rho=M_{W}^{2} / M_{Z}^{2} \cos ^{2} \theta_{W}$ yielding $m_{t}<203 \mathrm{GeV} / c^{2}$ (90\% CL).

It is therefore most likely that $m_{t}>M_{W}$, and the decay of the $t$-quark will proceed 
through a real, on-shell $W$ particle. If $m_{t}>100$ to $120 \mathrm{GeV} / c^{2}$, the $t$-quark will be inaccessible to the proposed LEP II, and the only hope for its discovery in the near future will be in $p \bar{p}$ collisions observed by CDF and D0 at the Tevatron with a large data sample of $50-100 \mathrm{pb}^{-1}$. After its discovery, detailed studies of the $t$-quark may require its study in $e^{+} e^{-}$annihilations, possibly requiring the construction of a linear $e^{+} e^{-}$collider [130] to achieve the necessary $E_{\text {mm. }}$. It should be noted that at these large masses, the resulting toponium state will be so wide that individual energy levels will be difficult to resolve.

A hesvy $t$-quark mass - ases interesting theoretical prospects since it would be in sharp contrast to the masses of all other known fermions. Quark mass matrices bave been proposed to predict both quark masses and the KM elements [131]. A heavy $t$-quark can be responsible for dynamically triggering electroweak symmetry breaking [132].

\subsubsection{Fourth Generation $b^{\prime}$-Quark}

The mass exclusion rarges for the $b^{\prime}$-quark are compared to previous and current, mass limits in Fig. 76. Again, ovr results are consistent with the slightly better LEP limits.

Almost all of the comments concerning the potential discovery of the $t$-quark can be applied to the $b^{\prime}$-quark. The $p \bar{p}$ collider t-quark limits are applicable to $b$ $\mathrm{CC}$ decay limits, but if $m_{b^{\prime}}<m_{t}$, then FCNC decays needed to be considered in the ture, particularly $b^{\prime} \rightarrow b Z$ if $m_{b^{\prime}}>M_{Z}$. Mixing angles or small mass differences can conspire to suppress fourth generation single quark decay modes, and fourth generation quarkonia may exist at high mass (unlike toponium). Anotbc: intriguing scenario is that fourth generation quarks may correspond to the "special" mass of $\approx 240 \mathrm{GeV} / c^{2}$ found by renormalizationgroup arguments [133]. Heavy fourth generation quarks, through new physics at a much higher mass scale, could also possibly generate the masses of the quarks of the first three generations. 


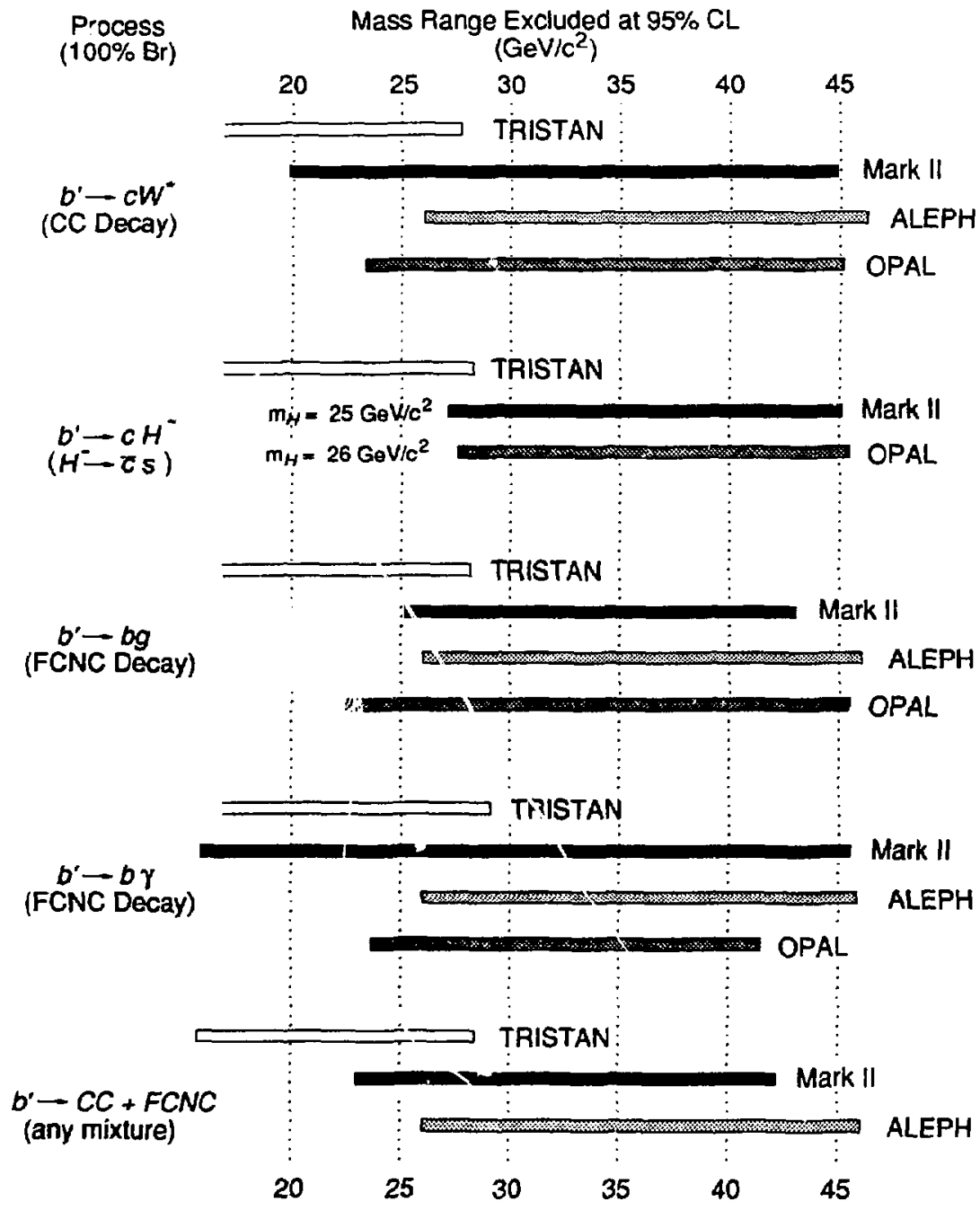

Figure 76: Summary and comparison of $b^{\prime}$-quark -ass limits from $e^{+} e^{-}$ colliders. The TRISTAN resuits existed at the time of the analysis, while the OPAL and ALEPH limits were published [125] shortly after the publication of the limits of this thesis. 


\subsubsection{Fourth Generation Neutrino $\nu_{4}$}

Figure 77 shows the relation between the exclusion regions for $\nu_{4}$ defined by the isolated track analysis, the normalized impact parameter analysis, and the measurement of $N_{\nu}$. It can also be seen that the previously excluded region has been significantly extended by this work. As seen in the figures of this chapter, the exclusion regions for mixing to the other generations for both Dirac and Majorana neutrinos are similar. Only AMY has also included limits on a Majorarz $\nu_{4}$. It has been noted that the CELLO measurement (region labelled (2) for $\ell=e$ in Fig. 77) did not include mixing to $\nu_{\tau}$, but this "hole" has been specifically eliminated by another analysis [134] of the Mark II $Z$ data. Therefore, for any mixing scenario for a Dirac $\nu_{4}$, the entire region bounded by $m_{\nu_{4}}<42.2 \mathrm{GeV} / c^{2}$ and $\left|U_{\ell 4}\right|^{2}>10^{-10}$ has now been excluded with the addition of the results of this thesis. Again, subsequent measurements by ALEPH with a larger sample of $Z$ decays [125] has resulted in a mone stringent exclusion region.

When the mass limits for stable neutrinos are combined with measurements from nonaccelerator experiments searching for dark matter [135], Dirac neutrinas as WIMP candidates Ior dark matter are categorically ruled out [136]. More stringent bounds on $N_{\nu}$ in the future from LEP will allow the exclusion of a host of other dark matter candidates, and exclude $m_{\nu_{4}}<M_{Z} / 2$ for any value of mixing to the other generations.

The search for unstable $\nu_{4}$ decaying through mixing can be continued at the Tevatron and future $p \bar{p}$ colliders because of its distinctive signature. However, for very massive stable neutrinos $\left(m_{\nu_{L}}>M_{Z} / 2\right)$, one o₹ the few possiblities for discovery is through 'neutrinocounting' by the detection of the single photon in the process $e^{+} e^{-} \rightarrow \nu \nu \gamma$ at very high energy $e^{+} e^{-}$colliders.

\subsubsection{Fourth Generation Charged Lepton $L^{-}$}

The exclusion region for a fourth generation charged lepton $L^{-}$is compared to other results in Fig. 78. This result covers a much larger region in the $m_{\nu_{L}}$ versus $m_{L}$ plane than previously reported. It should be stressed that the OPAL result is the only direct search from $Z$ decays. The indirect searches have the advantage of the ability to also exclude close-mass doublets (i.e. $m_{L} \approx m_{\nu_{L}}$ ). 


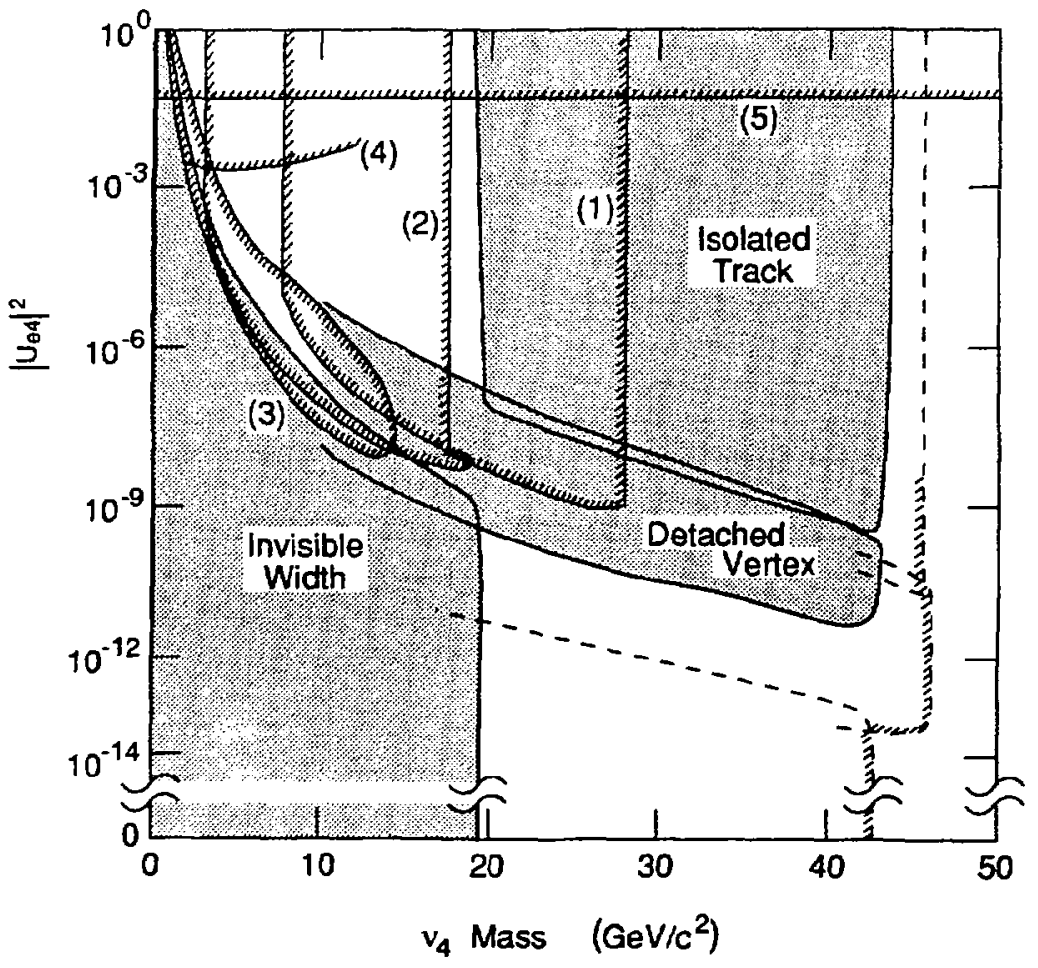

Figure 77: Mark II $\nu_{4} 95 \%$ exclusion regions (shaded areas) for a Dirac neutrino mixing exclusively with the first generation $(\ell=e)$. Examples of previous limits (solid, hatched lines) are given by (1) AMY [68], (2) CELLO [69], (3) Mark II secondary vertex search at PEP, [70], (4) monojet searches at PEP [71], and (5) $e-\mu$ universality [62]. Subsequent improved limits by ALEPH [125] are shown as dashed lines. 


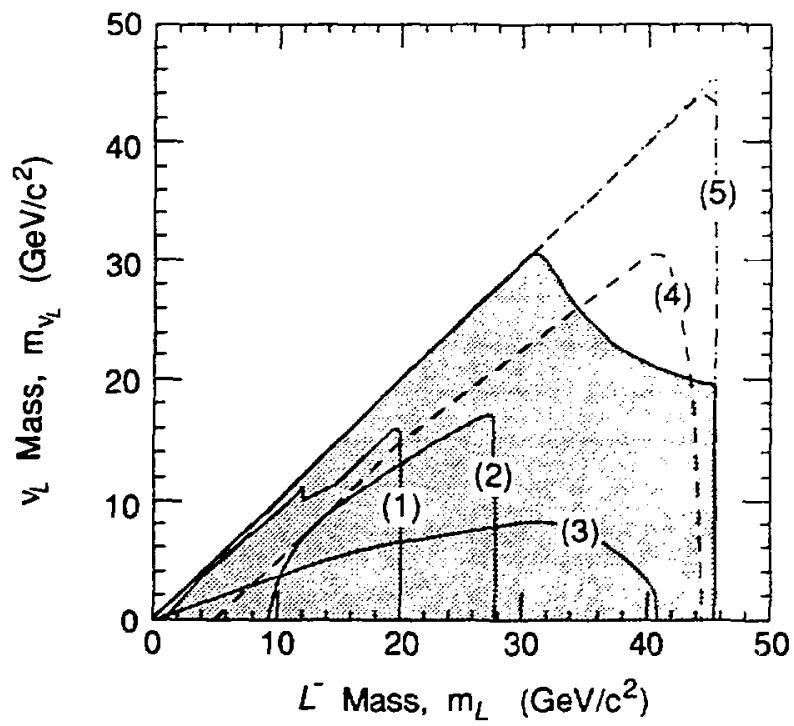

Figure 78: Mark II $m_{L}-m_{\nu_{L}} 95 \%$ exclusion region (shaded area, stable Dirac $\nu_{L}$.) Examples of previous limits (solid lines) are given by (1) PEP and PETRA [137], (2) TRISTAN [73], and (3) UA1 [74]. Subsequent improved limits by ALEPH [125] and OPAL (direct search) [138] are shown as dashed lines.

\subsection{Conclusions}

No evidence has been obeerved for $Z$ decays into $t$-quarks or fourth generation quarks and leptons considering both standard and a number of non-standard decays. These results substantially extend previous limits, and exclude a large mass range available for these new quarks and leptons from $Z$ decays. It is almost certain that, if they exist, all four members of the fourth generation have masses $>M_{Z} / 2$. In the first three generations, the neutrino has the lowest mass. If there is a fourth generation, then it is reasonable to expect that the $\nu_{4}$ still has the lowest mass. Therefore, the presented stringent limits on the mass of the $\nu_{4}$ cast further doubt on the possibility of a fourth generation. One can still hope that the small (if they are indeed nonvanishing) masses of the known neutrinos may be caused by the existence of a very massive $\nu_{4}$, as pastulated by some theories. All members could be very massive, but from limits on deviations of measured electroweal: parameters from 
theory predictions using radiative corrections, members of isospin doublets must be nearly degenerate in mass (see Eq. 47). This would be very different from the relatively large and growing difference between the mass of the b-quark and yet to be discovered $t$-quark.

If the fourth generation dows not exist, the generation puzzle still haunts us, albeit in a more restricted form:

Why are there three and only three generations of fundamental fermions? The plot thickens. 


\section{Bibliography}

[1] S.L. Glashow, Nucl. Phys. 22 (1961) 579;

S. Weinberg, Phys. Rev. Lett. 19 (1967) 1264;

A. Salam and J.C. Ward, Phys. Lett. 13 (1964) 168.

[2] UA1 Collaboration, G. Arnison et al., Phys. Lett. 126B (1983) 398;

UA2 Collaboration, P. Bagnaia et al., Phys. Lett. 129B (1983) 130.

[3] AMY Collaboration, T. Kumita et al., KEK-preprint 89-188, Feb. 1989, submitted to Phys. Rev. D,

VENUS Collaboration, K. Abe et al., Phys. Lett. 234B (1990) 382.

[4] ARguS Collaboration, H. Albrecht et al., Phys. Lett. 192B (1987) 245;

CLEO Collaboration, D.H. Miller, Purdue PU-89-643, presented at the European Physical Society International Conference on High Energy Physics, Madrid, Spain, Sep. 6-13, 1989.

[5] Mark II Collaboration, G.S. Abrams et al., Phys. Rev. Lett. 63 (1989) 2447;

Mark II Collaboration, C.-K. Jung, R. Van Kooten, et al., Phys. Rev. Lett. 64 (1990) 1091.

[6] M. Kobayashi and T. Maskawa, Progress of Theoretical Physics 49 (1973) 652.

[7] For example see F. Halzen and A. Martin, Quarks and Leptons: An Introductory Course in Modern Particle Physics, (John Wiley \& Sons, 1984).

[8] C. Quigg, Guage Theories of the Strong, Weak, and Electromagnetic Interactions, (Benjamin/Cummings, Menlo Park, 1983).

[9] Mark II Collaboration, S. Komamiya et al., Phys. Rev. Lett. 64 (1990) 1091. 
[10] W. Caswell, Phys. Rev. Lett. 33 (1974) 244;

Particle Data Group, M. Aguilar-Benitez et al., Phys. Lett. 170B (1986) .

[11] J. Schwinger, Particles, Sources and Fields, Vol. II, (Addison-Wesley, New York, 1973);

J. Jersak, E. Laermann, and P.M. Zervas, Phys. Lett. 88B (1981) 363;

T.H. Chang, K.J.F. Gaemers, and W.L. van Neerven, Nucl. Phys. B202 (1082) 407.

[12] S. Gusken, J.H. Kühn, and P.M. Zerwas, Phys. Lett. 155B (1985) 185.

[13] B.A. Kneihl and J.H. Kühn, Phys. Lett. 224B (1989) 229;

M. Dine and J. Sapirstein, Phys. Rev. Lett. 43 (1979) 668;

S.G. Gorishny, A.L. Kataev, and S.A. Larin, Phys. Lett. $212 B$ (1988) 238;

B.A. Kneihl and J.H. Kühn, MPI/PAE/PTh39/89.

[14] J. Alexander et al., Phys. Rev. D37 (1988) 56.

[15] G. Burgers et al., in $Z$ Physics at LEP, G. Altarelli, R. Kleiss, and C. Verzegnassi (eds.), CERN 89-08 (1989), Vol. 1, p. 55.

[16] G. Burgers and W. Hollik in Polarization at LEP, G. Alexarder et al.(eds.), CERN 88-06, Vol. 1, p. 136.

[17] H. Burkhandt et al, in Polarization at LEP, G. Alexander et al.(eds.), CERN 88-06, Vol. 1, p. 145.

[18] M. Consoli et al., in Z Physics at LEP, G. Altarelli, R. Kleiss, and C. Verzegnassi (eds.), CERN 89-08 (1989), Vol. I, p. 7;

[19] W. Marciano, Phys. Rev. D20 (1979) 274;

A. Sirlin, Phys. Rev. D22 (1980) 971.

[20] W. Marciano and A. Sirlin, Phys. Rev. D22 (1980) 2695;

W. Marciano and A. Sirlin, Phys. Rev. D29 (1984) 75, 945.

[21] D. Kennedy et al., Nucl. Phys. B321 (1989) 83;

D. Kennedy and B. Lynn, Nucl. Phys. B322 (1989) 1;

B.W. Lynn, Stanford Report SU-ITP-867 (1989). 
[22] W.J. Marciano and A. Sirlin, Phys. Rev. Lett. 46 (1981) 163;

A. Sirlin, preprint CERN-TH.5506/89 (1989).

[23] W. Hollik, preprint DESY 88-188 (1988);

W. Beenakker and W. Hollik, Zeit. Phys. C40 (1988) 141.

[24] Mark II Collaboration, G.S. Abrams et al., Phys. Rev. Lett. 63 (1989) 2173;

ALEPH Collaboration, D. Decamp et al., Phys. Lett. 231B (1989) 519;

DELPHI Collaboration, P. Aarnio et al., Ibid, 539;

L3 Collaboration, B. Adevia et al., Ibid, 509;

OPAL Collaboration, M. Akrawy et al., Tbid, 530;

[25] W. Hollik in Polarization at LEP, G. Alexander et al.(eds.), CERN 88-06, Vol. 1, p. 83.

[26] Ref. [32] and V. Khoze personal communication to Dr. A. Weinstein at the LeptonPhoton Symposium, and

V. Fadin, V Khoze, and T. Sjöstrand, CERN preprint CERN-TH-5687/90; V. Fadin and V. Khoze, JETP Lett. 46 (1987) 525.

[27] S.L. Wu, in Proceedings of the 1987 International Symposium on Lepton and Photon Interactions at High Energies, Hamburg, July 27-31, edited by W. Bartel and R. Ruckl, (North Holland, Amsterdam, 1988), p. 39;

B. Naraska et al., Phys. Rep. 148 (1987) 68.

[28] V. Barger and S. Pakvasa, Phys. Lett. 81B (1979) 195;

G. Kane and M. Peskin, Nucl. Phys. B195 (1982) 29.

[29] CLEO Collaboration, A. Bean et al., Phys. Rev. D35 (1987) 3533.

[30] For example, see J.C. Taylor, Gauge Theories of Weak Interactions.

[31] J.D. Bjorken, Phys. Rev. D17 (1978) 171;

M. Suzuki, Phys. Lett. 71B (1977) 189.

[32] J.H. Kühn, A. Reiter, and P.M. Zerwas, Nucl. Phys. B272 (1986) 560.

[33] C. Peterson et al., Phys. Rev. D27 (1983) 105.

[34] M. Bengtsson and T. Sjöstrand, Nucl. Phys. B289 (1987) 810. 
[35] J.H. Kühn and P.M. Zerwas, Max-Planck-Institut report MPI-PAE/PTh 52/87, 1987 (unpublished).

[36] M. Jezabek and J.H. Kühn, Phys. Lett. 207B (1988) 91.

[37] S.L. Glashow and S. Weinberg, Phys. Rev. D15 (1977) 1958;

H.E. Haber, G.L. Kane, and T. Sterling, Nucl. Phys. B161 (1979) 493.

[38] S.L. Glashow and E.E. Jenkins, Phys. Lett. 196B (1987) 233.

[39] CELLO Collaboration, H. Behrend et al., Phys. Lett. 193B (1987) 376.

[40] V. Barger and R.J.N. Phillips, Wisconsin preprint MAD/PH/497, May 1989.

[41] VENUS Collaboration, H. Yoshida et al., Phys. Lett. $198 B$ (1987) 570;

AMY Collaboration, H. Sagawa et al., Phys. Rev. Lett. 60 (1988) 93;

TOPAZ Collaboration, I. Adachi et al., Phys. Rev. Lett. 60 (1988) 97.

[42] UA1 Collaboration, C. Albajar et al., Zeit. Phys. C37 (1988) 505.

The results quoted in this reference have been analysed and updated by G. Altarelli, Nucl. Phys. B308 (1988) 724.

[43] CDF Collaboration, F. Abe et al., Phys. Rev. Lett. 64 (1990) 142 and 147.

[44] UA2 Collaboration, T. Alkesson et al., CERN preprint CERN-EP/89-152, Nov. 1989.

[45] V. Barger and R.J.N. Phillips, Phys. Lett. 201B (1989) 553.

[46] H. Harari and Y. Nir, Phys. Lett. 195B (1987) 586;

P. Krawczyk et al., Nucl. Phys. B307 (1988) 19.

[47] Global analyses of electroweak parameters before SLC and LEP $Z$ measurements:

U. Amaldi et al., Phys. Rev. D36 (1987) 1385;

G. Costa et al., Nucl. Phys. B297 (1988) 244.

[48] L. Wolfenstein, Phys. Rev. Lett. 51 (1983) 1945.

[49] V. Barger et al., Phys. Rev. D30 (1984) 947.

[50] W. Hou and R.G. Stuart, Phys. Rev. Lett. 62 (1989) 617.

[51] V. Barger, R.J.N. Phillips, and A. Soni, Phys. Rev. Lett. 57 (1986) 1518. 
[52] B. Haeri, A. Soni, and G. Eilam, Phys. Rev. Lett. 62 (1989) 719.

[53] I.J. Kroll, Ph.D. thesis, Harvard University.

[54] TOPAZ Collaboration, I. Adachi et al, Phys. Lett. 234B (1990) 197;

AMY Collaboration, S. Eno et al., Phys. Rev. Lett. 63 (1989) 1910;

VENUS Collaboration, K. Abe et al., Phys. Rev. D39 (1989) 3524.

[55] For recent reviews of neutrino masses see: S. M. Bilenky and S. T. Petcov, Rev. Mod. Phys. 59 (1987) 671;

B. Kayser and R. E. Scbrock, Phys. Lett. 112B (1982) 137.

[56] E. Majorana, Nuovo Cimento 14 (1937) 171.

For a particularly lucid description of the properties of massive neutrinos, see B. Kayser, F. Gibrat-Debu, and F. Perrier in The Physics of Massive Neutrinos, Notes in Physics Vol. 25 (World Scientific, Teaneck, N.J., 1989).

[57] M. Gell-mann, P. Ramond, and R. Slonsky in Supergravity, eds. D. Freedman and P. van Nieuwenhuizen (North Holland, Amsterdam, 1979) p. 315;

R. Mohapatra and G. Senjanovic, Phys. Rev. Lett. 44 (1980) 912;

R. Mohapatra and G. Senjanovic, Phys. Rev. D23 (1981) 165.

[58] E. Witten, Phys. Lett. 91B (1980) 81;

R. Mohapatra and G.Senjanovic, Phys. Rev. Lett. 44 (1980) 912;

A. Davidson and K. Wali, Phys. Lett. 98B (1981) 183;

E. Papatonopoulos and G. Zoupanos, Phys. Lett. 110B (1982) 465;

J. Bagger and S.Dimopolous, Nucl. Phys. 244 (1984) 247;

J. Bagger et al., Phys. Rev. Lett. 54 (1985) 2199.

[59] B.W. Lee and S. Weinberg, Phys. Rev. Lett. 39 (1977) 165;

D. Dicus et al., Phys. Rev. Lett. 39 (1977) 168.

[60] V. Barger, W.Y. Keung, and R.J.N. Phillips, Phys. Lett. $141 B$ (1984) 126.

[61] S.L. Glashow, J. Miopoulos, and L. Maiani, Phys. Rev. D2 (1980) 912;

S.L. Glashow and S. Weinberg, Phys. Rev. D15 (1977) 1958;

E.A. Paschos, Phys. Rev. D15 (1977) 1966.

[62] M. Gronau, C.N. Leung, and J.L. Rosner, Phys. Rev. D29 (1984) 2539. 
[63] M. Dittmar et al., CERN preprint CERN EP/89-50; April 1989.

[64] Mark II Collaboration, E. Soderstrom, J.A. McKenna, et al., SLAC preprint SLACPUB-5192, Feb. 1990, submitted to Phys. Rev. Lett.

[65] R.E. Schrock, Phys. Rev. D24 (1981) 1275;

Y.S. Tsai, Phys. Rev. D4 (1971) 2821;

Phys. Rev. D19 (1979) 2809.

[66] Mark II Collaboration, G.S. Abrams et al., Phys. Rev. Lett. 63 (1989) 724;

Mark II Collaboration, G.S. Abrams et al., Phys. Rev. Lett. 63 (1989) 2173;

J. Nash, Ph.D. thesis, Stanford University, SLAC-355, January 1990.

[67] F.J. Gilman, Comments Nucl. Part. Phys. 16 (1986) 231.

K.K. Can and M.L. Perl, Int. Jour. Mod. Phys. A3 (1988) 531.

[68] AMY Collaboration, N.M. Shaw et al., Phys. Rev. Lett. 63 (1989) 1342.

[69] CELLO Collaboration, H.-J. Behrend et al., Zeit. Phys. C41 (1988) 7.

[70] Mark II Collaboration, C. Wendt et al., Phys. Rev. Lett. 68 (1987) 1810.

[71] F.J. Gilman and S.H. Rhie, Phys. Rev. D32 (1985) 324.

[72] J.K. Riles, Ph.D. thesis, Stanford University, SLAC-342, April 1989;

D.P. Stoker et al., Phys. Rev. D39 (1989) 1811.

[73] AMY Collaboration, G.N. Kim et al., Phys. Rev. Lett. 61 (1988) 911;

TOPAZ Collaboration, I. Adachi et al, Phys. Rev. D37 (1988) 1339;

VENUS Collaboration, K. Abe et al., Phys. Rev. Lett. 61 (1988) 915.

[74] UAl Collaboration, C. Albajar et al., Phys. Lett. 185B (1987) 241.

[75] SLAC Linear Collider Conceptual Design Report, SLAC Report SLAC-229, June 1980.

[76] W. Kozanecki, SLAC preprint SLAC-PUB-4859, Feb. 1989;

B. Richter, SLAC preprint SLAC-PUB-5075, Aug. 1989.

[77] R. Jacobsen et al., SLAC preprint SLAC-PUB-5205, Apr. 1989. 
[78] J Kent et al., SLAC preprint SLAC-PUB-4922, Mar. 1989:

M. Levi et al., SLAC prepridt. SI.AC-PUB-5110, Mar. 1989.

[79] M. Levi, J. Nash, and S Watson, Nucl. Instr. Meth. A281 (1989) 265.

[80] G.S. Abrams et al., Nucl. Instr. Meth. A.281 (1989) 55.

[81] G. Bowden et al., Nucl. Instr. Meth. A278 (1989) 664.

[82] D. Dernstein et al., IEEE Traus. Nucl. Sci. NS-33 (1986) 86.

[83] H. Brafman et al., IEEE Trans. Nucl. Sci. NS-32 (1985) 336.

[84] J. Perl et al., Nucl. Instr. Meth. A252 (1986) 616.

[85] D. Schaile et al., Nucl. Instr. Meth. A242 (1986) 247.

[86] The value was obtained from Eric Wicklund, private communication.

[87] A. Boyarski et al., Nucl. Instr. Meth. A283 (1989) 617.

[88] G.S. Abrams et al., IEEEE Trans. Nucl. Sci. NS-25 (1978) 309.

G.S. Abrams et al., IEEE Trans. Nucl. Sci. NS-27 (1980) 59.

[89] E. Cisneros et al, IEEE Trans. Nucl. Sci. NS-24 (1977) 413.

[90] M. Breidenbach et al., IEEE Trans. Nucl. Sci. NS-25 (1978) 703.

[91] C. Blocker, Ph.D. thesis, University of California, Berkeley, LBL-10801 (1980).

[92] J.A. Kadyk, Proceedings of the Gas Sampling Calorimetry Workshop II, Batavia, IL (1985) 373.

[93] M. Petradza et al., Mark II-SLC Note \#164 (1986), unpublished.

[94] S. Hong, Ph.D. thesis, University of Michigan.

[95] H. Brafman et al., SLAC preprint SLAC-PUB-2033, October 1977;

T. Himel, Ph.D. thesis, Stanford University, SLAC-REP-223, October 1979.

[96] R. Aleksan et al., Proceedings of the International Conference on the Impact of Digital Microelectronics and Microprocessors on Particle Physics, World Scientific (1988) 38. 
[97] W.R. Nelson et al., SLAC-REP-265, December 1985.

[98] J. Nash, Ph.D. thesis, Stanford University, SLAC-356, January 1990.

[99] D.J. Nelson et al., IEEE Trans. Nucl. Sci. NS-28 (1981) 336.

[100] A.J. Lankford and T. Glanzman, IEEE Trans. Nucl. Sci. NS-31 (1984) 225;

T. Barklow et al., IEEE Trans. Nucl. Sci. NS-33 (1986) 775.

[101] T. Sjöstrand and M. Bengtsson, Comput. Phys. Commun. 43 (1987) 367;

M. Bengstsson and T. Sjöstrand, Nucl. Phys. B289 (1987) 810;

B. Anderssen et al., Phys. Rept. 97 (1983) 33.

[102] F. Gutbrod, G. Kramer, and G. Shierholz, Zeit. Phys. C21 (1984) 235;

T.D. Gottschalk and M.P. Shatz, Phys. Lett. 150B (1985) 451.

[103] P. Mättig, Phys. Rept. 177 (1989) 141.

[104] Mark II Collaboration, A. Petersen et al., Phys. Rev. D37 (1988) 1.

[105] Kathryn O'Shaughnessy, Ph.D. Thesis, Stanford University.

[106] G. Marchesini and B.R. Webber, Nucl. Phys. B238 (1984) 1;

B.R. Webber, Nucl. Phys. B238 (1984) 492;

G. Marchesini and B.R. Webber, Nucl. Phys. B310 (1988) 461.

[107] R.D. Field and S. Wolfram, Nucl. Phys. B213 (1983) 65 and Ref. [106].

[108] D.P. Stoker, Mark II/SLC Note \#161 (November 1986) (unpublished); A thorough description can also be found in the sppendix of Ref. [72], and in D.P. Stoker et al., Phys. Rev. D39 (1989) 1811.

[109] Modifications to LULEPT for the generation and decay of $\nu_{4}$ have been performed by $D$. Stoker and the author.

[110] E. Ma and J. Pantaleaone, Phys. Rev. D40 (1989) 2172.

[11] P.A. Aarnio et al., CERN-TIS-RP/168, 1986;

J. Ranft, H.-J. Möhring, T.M. Jenkins, and W.R. Nelson, SLAC-TN-86-3, April 1986. 
[112] F.A. Berends, P.H. Daverveld, and R. Kleiss, Comp. Phys. Comm. C 40 (1986) 309.

[113] T. Barklow, SLAC Report SLAC-315 (July 1987) 210 (unpublished).

[114] JADE Collaboration, W. Bartel et al, Zeit. Phys. C33 (1986) 23.

[115] A.G. Frodesen, O. Skjeggestad, and H. Tofte, Probability and Statistics in Particle Physics, (Universitetsforlaget, Oslo, 1979).

[116] G. Hanson, SLAC Report SLAC-315 (July 1987) 196 (unpublished). This reference is also a comprehensive survey of other search methods for the $t$-quark.

[117] C. Hearty, Mark II/SLC Note \#230, May 1989 (unpublished).

[118] Personal communication, M. Woods, Nov. 1989.

[119] S. Klein, Mark II/SLC Physics Working Group Note \#5-2 (unpublished), A. Weinstein, Mark II/SLC Physics Working Group Note \#5-1 (unpublished).

[120] R.N. Cahn, Phys. Rev. D36 (1987) 2666. The difference between this analytical form of the $Z$ line shape and that given by more complete Monte Carlo calcr' ' ns has been found to be less than $1 \mathrm{MeV} / c^{2}$ for $M_{Z}$, less than $10 \mathrm{MeV}$ for the toud width, and less than $0.01 \%$ in $\sigma_{\text {peak }}$.

[121] KORALZ Monte Carlo, S. Jadach and Z. Was, Comput. Phys. Commun. C 36 (1985) 197.

[122] W.H. Press et al., Numerical Recipes, (Cambridge University Prese, 1986) p. 77-89.

[123] Particle Data C c , Phys. Lett. 204B (1988) 81;

O. Helene, Nucl. Instr. Meth. 212 (1983) 319.

[1'24] Mark II Collaboration, G.S. Abrams et al., Phys. Rev Lett. 63 (1989) 1558. In this messurement, the JADE clustering algorithm is used to define and count jetr. For a value of $y_{\text {cut }}=0.015$, roughly $0.5 \%$ of the hadronic $Z$ decays are found to be five jet events. This value of $y_{\text {cut }}$ is roughly equivalent to the value of $y_{\text {ruin }}=0.015$ (the minimum scaled invariant mass two partons must have to prevent them from being combined into one) in the LUND matrix element model. 
[125] ALEPH Collaboration, D. Decamp et al., Phys. Lett. 236B (1990) 511;

DELPHI Collaboration, P. Aarnio et al., CERN preprint CERN-EP/90-49 (submitted to Phys. Lett. B);

OPAL Collaboration, M.Z. Akrawy et al., Phys. Lett. 236B (1990) 364.

[126] Y. Nir. Phys. Lett. 236B (1990) 471.

[127] UAl Collaboration, M. Felcini et al., CERN preprint CERN-EP/89-173.

[128] DELPHI Collaboration, P. Aarnio et al., CERN preprint CERN-EP/90-33, Feb. 1990;

OPAL Coilaboration, M Akraway et al., CERN preprinc CERN-EP/90-38, Mar. 1990.

[129] S.N. Ganguli and A. Gurtu, TIFR-EHEP 90/1, Feb. 1990.

[130] C. Ahn et al., SLAC Report SLAC-329, May 1988 (unpublished).

[131] H. Fritzsch, Phys. Lett. $73 B$ (1978) 317;

M. Gronsu and J. Shecter, Phys. Rev. D37 (1988) 2597.

[132] Alvarez-Gaumé, J. Polchinski, and M.B. Wise, Nucl. Phys. B221 (1983) 495;

W.J. Marciano, Phys. Rev. Lett. 62 (1989) 2793.

[133] B. Pendleton and G. Ross, Phys. Lett. 98B (1981) 291;

C. Hill, Phys. Rev. D24 (1981) 691.

[134] P. Burchat, M. King et al., SLAC preprint SLAC-PUB-5172, Feb. 1990 (to be published in Rapid Communications, Phys. Rev. D).

[135] D. Caldwell et al, Phys. Rev. Lett. 61 (1988) 510;

S. Ablen et al., Phys. Lett. 195B (1987) 603.

[136] L.M. Krauss, Phys. Rev. Letı. 64 (1990) 999.

[137] S.L. Wu, in Proceedings of the 1987 International Symposium on Lepton and Photon Interactions at High Energies, Hamburg, July 27-31, edited by W. Bartel aud R. Ruckl, (North Holland, Amsterdam, 1988), p. 39.

[138] OPAL Collaboration, M.Z. Akrawy et al., CERN preprint CERN-EP/90-09, Jan. 1990 (submitted to Phys. Lett. B). 